\title{
Training cognitive control
}

Citation for published version (APA):

Dassen, F. C. M-L. (2018). Training cognitive control: the role of executive functioning in eating behavior and weight loss. [Doctoral Thesis, Maastricht University]. Ridderprint BV.

https://doi.org/10.26481/dis.20180711fd

Document status and date:

Published: 01/01/2018

DOI:

10.26481/dis.20180711fd

Document Version:

Publisher's PDF, also known as Version of record

\section{Please check the document version of this publication:}

- A submitted manuscript is the version of the article upon submission and before peer-review. There can be important differences between the submitted version and the official published version of record.

People interested in the research are advised to contact the author for the final version of the publication, or visit the DOI to the publisher's website.

- The final author version and the galley proof are versions of the publication after peer review.

- The final published version features the final layout of the paper including the volume, issue and page numbers.

Link to publication

\footnotetext{
General rights rights.

- You may freely distribute the URL identifying the publication in the public portal. please follow below link for the End User Agreement:

www.umlib.nl/taverne-license

Take down policy

If you believe that this document breaches copyright please contact us at:

repository@maastrichtuniversity.nl

providing details and we will investigate your claim.
}

Copyright and moral rights for the publications made accessible in the public portal are retained by the authors and/or other copyright owners and it is a condition of accessing publications that users recognise and abide by the legal requirements associated with these

- Users may download and print one copy of any publication from the public portal for the purpose of private study or research.

- You may not further distribute the material or use it for any profit-making activity or commercial gain

If the publication is distributed under the terms of Article $25 \mathrm{fa}$ of the Dutch Copyright Act, indicated by the "Taverne" license above, 


\section{TRAINING COGNITIVE CONTROL}

The role of executive functioning in eating behavior and weight loss

Fania Cher Marie-Louise Dassen 
The research presented in this dissertation was funded by the Maastricht University Interfaculty Program 'Eatwell'

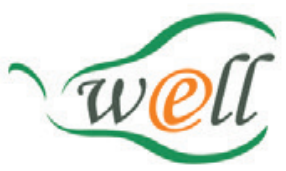

Cover Anne Lennartz

Lay-out Nikki Vermeulen | Ridderprint BV

Printing Ridderprint BV | www.ridderprint.nl

ISBN 978-94-6375-006-6

(c) Fania Cher Marie-Louise Dassen, 2018

No parts of this publication may be transmitted, in any form or by any means, without permission of the author. 


\title{
Training cognitive control \\ The role of executive functioning in eating behavior and weight loss
}

\author{
PROEFSCHRIFT \\ Ter verkrijging van de graad van doctor aan de Universiteit Maastricht, \\ op gezag van de Rector Magnificus, Prof. dr. Rianne M. Letschert, \\ volgens het besluit van het College van Decanen, \\ in het openbaar te verdedigen op
}

11 juli 2018 om 12 uur

door

Fania Cher Marie-Louise Dassen 


\section{Promotor}

Prof. Dr. A. Jansen

\section{Copromotoren}

Dr. K. Houben

Dr. C. Nederkoorn

\section{Beoordelingscommissie}

Prof. Dr. A.M.W.J. Schols (voorzitter)

Prof. Dr. C. Braet - Universiteit Gent

Prof. Dr. R.W. Holland - Radboud Universiteit Nijmegen

Dr. P.P.M. Hurks

Prof. Dr. G.J. Kok 


\section{TABLE OF CONTENTS}

$\begin{array}{lll}\text { Chapter } 1 & \text { General Introduction } & 7\end{array}$

PART 1. The Cognitive Profile of Obesity

Chapter 2 Self-regulation and Obesity: The Role of Executive Function and Delay Discounting in the Prediction of Weight Loss

Chapter 3 Time Orientation and Eating Behavior: Unhealthy Eaters

Consider Immediate Consequences, while Healthy Eaters Focus on Future Health

\section{PART 2. Manipulating Cognitive Control}

Chapter 4 Focus on the Future: Episodic Future Thinking Reduces

Discount Rate and Snacking

Chapter 5 Manipulating Cognitive Flexibility: Can we Change Eating

Behavior through Training of Executive Control?

Chapter 6 Gamified Working Memory Training in Overweight

Summary

Samenvatting

Valorisation Addendum

Dankwoord

Curriculum Vitae

References 



\section{CHAPTER}

General Introduction

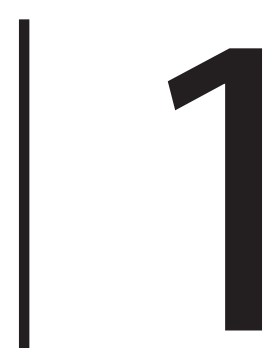



'Eat less, eat better, exercise more: change your lifestyle!' Most of the obese do know this and if they could, obesity was not a problem - Prof. Anita Jansen

Obesity is a growing problem worldwide. The prevalence of overweight and obesity has more than doubled in the past 30 years (Flegal, Carroll, Kit, \& Ogden, 2012). To illustrate: in 2014, about 1.9 billion adults worldwide were overweight, of which 600 million were obese (WHO, 2018). Categories of normal weight, overweight, and obesity are defined using the body mass index (BMI). The BMI is a ratio of weight to height and calculated as $\mathrm{kg} / \mathrm{m}^{2}$. A BMI between 18.5 and 24.9 is considered a healthy BMI, a BMI between 25 and 29.9 is classified as overweight, and a BMI of 30 and above is classified as obesity (WHO, 2018). Excessive body weight can have detrimental consequences for our health. For instance, overweight and obesity increase the risk of several chronic diseases, including diabetes, cardiovascular disease, musculoskeletal disorders, and it makes someone more susceptible to certain forms of cancer (Lim et al., 2013). In addition to these medical consequences, obesity is also associated with serious psychological consequences such as a reduced quality of life, by for instance stigma, weight discrimination, depression and mobility problems (Uzogara, 2017). At the moment, overweight and obesity are associated with a higher number of deaths worldwide than underweight (WHO, 2018).

Obesity is in essence the result of a simple equation: when energy intake exceeds energy expenditure, weight will be gained. A large part of individual differences in BMI seems to be caused by behavioral factors (Ravussin \& Bogardus, 2000). One contributing factor to overweight and obesity is the fact that people enjoy the taste of foods high in sugar and fat (Drewnowski \& Greenwood, 1983). This could be seen as an evolutionary benefit for survival during times of food scarcity. However, in the current environment this is working against us: nowadays we live in this so-called 'obesogenic environment', which is "the sum of influences that the surroundings, opportunities, or conditions of life have on promoting obesity in individuals or populations" (Swinburn, Egger, \& Raza, 1999 , p. 564). We are surrounded by food cues that remind us of food intake, like the smell or taste of foods (Boswell \& Kober, 2016). This obesogenic environment seems to trigger frequent and unhealthy food intake (Hill \& Peters, 1998). A healthy eating pattern is considered one with limited energy intake from fats and sugars, and high in consumption of fruit, vegetables, legumes, whole grains and nuts (WHO, 2018). However, high-caloric foods are aggressively marketed and almost always available, and constantly choices have to be made about when to eat, what to eat and how much to eat. Besides this abundance in high-caloric foods, a sedentary lifestyle is promoted in our current environment: many people go to work by car, to the right floor by elevator, and they are sitting behind their computer all day. Energy expenditure is therefore quite low for most people. This obesogenic, food-abundant environment full of temptations 
and without the need for hard labor or exercise makes it difficult to maintain a healthy energy balance (Hill \& Peters, 1998).

To achieve weight loss, one needs to reduce caloric intake, increase psychical activity, or do both; though note that research shows that in order to lose weight, dietary changes are in general more effective than increases in physical activity (Dunn et al., 2006). Ultimately, it does not matter what diet someone choses: all caloric-restriction diets appear to work, as long as someone is able to persevere (Johnston et al., 2014). At the moment, the common treatment for weight loss is lifestyle intervention. The term lifestyle intervention consists of the main components of diet, exercise, and counseling (Wadden, 2003; Wadden, Butryn, \& Byrne, 2004; Wing, 2004). The goal is to achieve a negative energy balance via increased physical activity and a reduction in daily energy intake (Wadden et al., 2004). Even a small percentage of weight loss already has beneficial effects on our health (Goldstein, 1992). Clinically important weight loss is in general defined as a loss of $5-10 \%$ of baseline weight (Blackburn, 1995; Goldstein, 1992). Current lifestyle interventions for weight loss, however, do not prove to be very successful (Wadden et al., 2004). In general, they do lead to some weight loss in the short term, but this weight loss is rarely maintained over a longer period of time (Mann et al., 2007; Meule \& Vögele, 2017). Considering the impact of obesity on the population's health, it is important to find out which psychological elements should be targeted to improve treatment success.

\section{Theoretical models of self-regulation and health behavior The intention-behavior gap}

Most people do know they should eat less and exercise more in order to lose weight. Unfortunately, it is not the case that if someone knows what to do, he or she will also automatically behave like this (Sheeran, 2002). Often an attempt to lose weight starts with the intention to go on a diet. Intentions are instructions that people give to themselves to behave in a certain way, inspired by an underlying motivation to perform a certain behavior (Sheeran, 2002). It is suggested that intentions are the best predictor for the performance of behavior (Ajzen, 1991). However, for instance, Mullan, Allom, Brogan, Kothe, and Todd (2014) showed that intention explained about $25 \%$ of the variance in self-reported saturated fat intake and $9-17 \%$ of the variance in fruit and vegetable consumption, leaving a great deal of variation unexplained. Intention is also not very helpful when predicting impulsive or automatic behaviors (Sheeran, 2002). Thus, there is a gap between intention and the actual behavior, the so-called intentionbehavior gap, and only considering intention provides an incomplete explanation for behavior (Sheeran, 2002). Webb and Sheeran (2006) conducted a meta-analysis to see how strongly intention and behavioral change were associated. They concluded that 
most interventions did lead to major changes in intention, but only to minor changes in behavior. Thus, it seems not clear what is causing this intention-behavior gap.

\section{Bridging the gap: Dual process theories}

\section{The Reflective-Impulsive model}

In closing this gap between behavior and intentions, dual process models provide a helpful framework. Dual process theories divide mental processes into two different information-processing systems depending on whether they operate automatically or in a controlled manner (Gawronski \& Creighton, 2013). In order to reach our long-term goals, we need to regulate our behavior and control our impulses. Self-regulation is in general defined as the willful regulation of cognition and behavior in order to achieve long-term goals (Baumeister, Vohs, \& Tice, 2007). One of the most influential theories in the field is the Reflective-Impulsive model (Hofmann, Friese, \& Strack, 2009; Strack \& Deutsch, 2004). This framework describes the reflective-impulsive network as a parallelcompetitive system (Strack \& Deutsch, 2004). The Reflective-Impulsive model states that our behavioral decisions are made on the one hand by the reflective system which is based on reasoning and goals, and on the other hand by the impulsive system which is based on associations. These two systems interact at various stages of information processing and decision making, and both systems access a common final mechanism for overt behavior execution. When someone is on a diet, these systems could favor different outcomes, resulting in a self-control conflict. A dieter wants to restrict caloric intake in favor of long term weight loss, though the perception of a tempting snack could automatically activate an approach bias. Which behavioral schema (impulsive or reflective) wins over the other in this situation depends on the relative strength of activation (i.e., winner-takes-all response). As the two systems operate differently, certain situational and dispositional boundary conditions could shift activation in favor of one system, resulting in this system being more dominant than the other. Thus, health behaviors are determined by both the reflective and impulsive system and their degree of influence on behavior is moderated by various boundary conditions (Hofmann, Friese, \& Strack, 2009; see Figure 1 for a graphical display of the Reflective-Impulsive model). One assumption underlying these boundary conditions is that actions of the reflective system are dependent on control resources. Certain moderators restrict the availability of control resources and selectively impair the reflective system. As a result, individuals are more likely to act upon their impulses when their control resources are low, and less likely to act upon their impulses when resources are high (Hofmann, Schmeichel, \& Baddeley, 2012). Boundary conditions could be, for instance, situational factors like fatigue or motivation, or more innate factors like executive function (EF). EF is defined as a set of higher level, 'supervisory' cognitive functions involved in the 
control and regulation of more basic, lower-level cognitive processes and goal-directed, future-oriented behavior (Alvarez \& Emory, 2006). According to the Reflective-Impulsive model, EF is necessary for ensuring that the goals of the reflective system are reached by controlling or overriding the impulsive system. Thus, with ineffective EF, impulses are more likely to determine behavior. To illustrate this, there is ample evidence showing a stronger influence of automatic, impulsive processes and a weaker influence of goals and intentions on behavior when EFs (or cognitive resources in general) are weak, whereas this picture is reversed when EFs are strong (e.g. Friese, Hofmann, \& Wänke, 2008; Hofmann, Friese, \& Roefs, 2009; Houben \& Wiers, 2009).

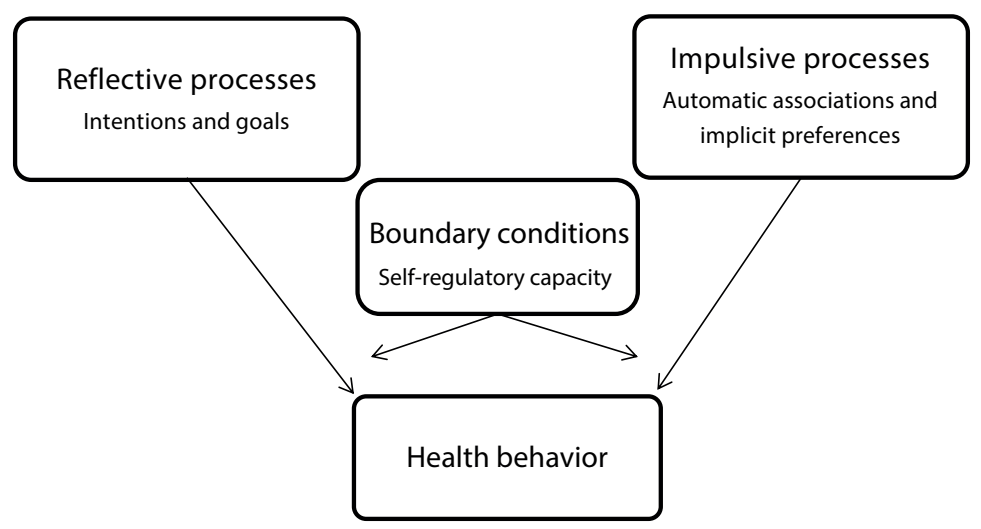

FIGURE 1 Reflective-Impulsive model of goal-directed behavior (adapted from Hofmann, Friese, \& Strack, 2009).

\section{The Competing Neurobiological Decision Systems theory}

The Competing Neurobehavioral Decision Systems theory (CNDS; Bickel et al., 2007; Bickel \& Yi, 2008) is a recent dual systems model of addiction that can also be applied to other unhealthy behaviors that deliver immediate gratification and primarily longterm costs, including obesity. The CNDS theory assumes that behavior is the product of two competing neural systems: the reward-driven impulsive decision system and the executive decision system (see Figure 2). This theory is basically the neurological counterpart to cognitive dual-process theories like the Reflective-Impulsive model. When both systems are in balance, as indicated by the diagonal line in Figure 2, individuals can flexibly modulate their behavior and make appropriate decisions. However, an unbalance between these systems produces maladaptive decision making. In this theory, then, maladaptive health behaviors like overeating are the result of a hyperactive impulsive system and/or hypoactive executive system. A collapse of selfcontrol can occur because of both insufficient top-down control and overwhelming 
bottom-up impulses. However, with sufficient executive control individuals are able to continue carrying out their healthy behavior intentions despite tempting alternatives (Epstein, Salvy, Carr, Dearing, \& Bickel, 2010). This is similar to this EF capacity being considered a boundary condition in the Reflective-Impulsive model. High caloric foods provide a source of reinforcement, and to an individual with a relatively stronger impulsive system, these temptations are hard to resist. Important is the role of reinforcement pathology, which refers to the combined effects of a high valuation for immediate reinforcers (food is considered highly rewarding) and an excessive preference for immediate acquisition of a reinforcer despite negative long-term consequences (i.e., delay discounting; a cognitive variable explained later in more detail). Thus, rewards become even more tempting when immediately available (Epstein et al., 2010), a situation often encountered in our obesogenic environment involving the abundant presence of high-caloric foods.

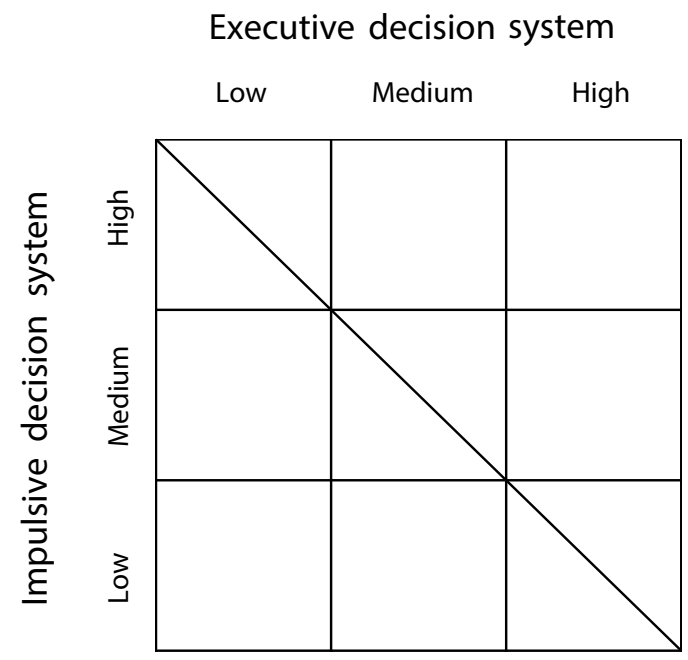

FIGURE 2 Competing Neurobiological Decision Systems theory of decision making (adapted from Bickel et al., 2007). The diagonal line indicates a balance between both systems, enabling appropriate decision making.

\section{Cognitive components involved in overeating Executive function (EF)}

Both the Reflective-Impulsive model and the CNDS theory point out a significant role of $\mathrm{EF}$ in self-regulation, prompting questions about the role of EF in weight management. EFs, a set of cognitive processes, are necessary for the top-down cognitive control of behavior; our ability to regulate ourselves to reach future goals is also referred to 
as cognitive control. EFs help us to select and monitor behaviors that facilitate goal achievement. There are three main facets of EF generally distinguished in the literature: inhibition, working memory and shifting (Diamond, 2013; Miyake et al., 2000). EF is a complex, multifaceted construct, and these main facets of EF are considered distinct, yet related constructs (Miyake \& Friedman, 2012; Miyake et al., 2000). These three main facets of EF support complex self-regulatory skills, such as problem solving, planning, and decision making (Epstein, Valoski, Wing, \& McCurley, 1994). EF can be seen as our 'control system' and ensures that we, for example, are able to focus on long-term weight loss goals, can control food-related thoughts, resist temptations and regulate responses to food stimuli, which are all considered important components of effective dieting. EF seems important to manage food intake and physical activity in our current obesogenic environment (Appelhans, French, Pagoto, \& Sherwood, 2016; Jansen, Houben, \& Roefs, 2015). Without EF we would be at the mercy of our impulses, unable to resist temptations. We specifically need EFs in new, unfamiliar circumstances or when we want to change our behavior; they are not critical for daily routine or well-learned behaviors (Shallice \& Vallar, 1990). Thus, EF could play an important role in the development and maintenance of obesity due to their role in self-regulatory processes that manage our energy-balance. Below, the three core facets of EF are described in more detail and their proposed link with obesity is explained.

\section{Inhibition}

Inhibition, also referred to as inhibitory control, can be defined as the ability to override or interrupt undesired behavioral responses (Logan, Cowan, \& Davis, 1984). Inhibition helps us to stay focused despite distraction, and to inhibit impulsive actions triggered by the current obesogenic environment. The Stop-Signal Task (SST; Logan, Schachar, \& Tannock, 1997) and Go/No-Go task (GNG; Miller, Schäffer, \& Hackley, 1991) are commonly used behavioral tasks to measure inhibition. The SST consists of go and stop trials. Participants are instructed to respond as fast as possible to the go stimulus. However, if a stop signal is presented, with some delay after the go stimulus, the participant has to inhibit or overrule his or her already initiated response. In the GNG, participants are required to either respond or withhold their response depending on whether a go stimulus or a nogo stimulus is presented. In both tasks one has to inhibit the processing of a bottom-up response. Numerous studies have shown associations between weaker inhibition and obesity (for reviews see Bartholdy, Dalton, O’Daly, Campbell, \& Schmidt, 2016; Lavagnino, Arnone, Cao, Soares, \& Selvaraj, 2016). However, it is has been suggested that overweight and obese individuals do not show weaker inhibition in all areas, but mainly experience more difficulty inhibiting responses specific to food (Bartholdy et al., 2016; Houben, Nederkoorn, \& Jansen, 2014; Nederkoorn, Coelho, Guerrieri, Houben, \& Jansen, 2012). 


\section{Working memory}

Working memory, also known as updating, is considered a limited capacity system that temporarily holds important information in mind while we are mentally manipulating it (Baddeley, 1992). Working memory is, for instance, important to focus our attention (Barrett, Tugade, \& Engle, 2004) and to keep a mental representation of long-term goals active (Hofmann, Gschwendner, Friese, Wiers, \& Schmitt, 2008). Originally, working memory was divided into three main elements. The central executive is considered an attentional control system responsible for allocating attention across two slave subsystems (Baddeley, 1992). The visuospatial sketchpad is a limited-capacity slave systems and responsible for manipulating and storing visual and spatial information. The other slave system is the phonological loop, which is responsible for manipulating and storing speech-based information. A fourth component has been added to the model, the episodic buffer, which is a limited capacity passive system integrating units of information (Baddeley, 2000). A task commonly used to measure working memory is the n-back task (Kirchner, 1958). Participants are instructed to monitor a series of stimuli and they have to indicate whether the stimulus that is presented is the same as the one presented $n$ trials ago. This task requires on-line monitoring, updating, and manipulation of remembered information, and is therefore considered as a complex working memory task, measuring key processes within working memory (Owen, McMillan, Laird, \& Bullmore, 2005). Research has shown associations between less effective working memory and an elevated BMI (e.g. Gunstad et al., 2007; Smith, Hay, Campbell, \& Trollor, 2011). Working memory could, for instance, be important to maintain weight loss goals in an active state to efficiently monitor food intake (Boutelle \& Kirschenbaum, 1998). Working memory is considered as a distinct construct, though it is also highly interrelated to the other main facets of EF (Friedman \& Miyake, 2017; Miyake \& Friedman, 2012; Miyake et al., 2000). For instance, maintaining a goal and remembering what you should and should not do to reach it (working memory) is critical for knowing what behavior to inhibit (inhibition), and the other way around, inhibiting environmental and internal distractions is critical for staying focused on our goals.

\section{Shifting}

Shifting, also known as task switching or cognitive flexibility, is the third main facet of $\mathrm{EF}$ and entails being able to easily and quickly switch between perspectives or the focus of attention, flexibly adjust to changed demands or priorities, and think outside the box (Diamond, 2013). It also involves overcoming interference from a previous task, thereby avoiding perseverations to an old behavior when this no longer fits a new goal (Miyake et al., 2000). The Trail Making Test (TMT; Reitan, 1958) and the Wisconsin Card Sorting Test (WCST; Grant \& Berg, 1948) are commonly used measures to asses shifting. 
In the TMT, first in part A participants have to draw as fast as possible consecutive lines between numbers in an ascending pattern, whereas in part $B$ they have the added task of alternating between numbers and letters. Shifting ability is assessed as the time to complete part B minus the time to complete part A (Reitan, 1958). In the WCST, participants are asked to sort a pile of cards by matching them to one of four cards displayed, but they are not told how to do this. After every sorting, the participant receives feedback whether this match was correct or incorrect. During the task, the sorting rule changes without the participant being informed. The participant has to shift sets accordingly and sort cards following the new sorting rule, without persevering to the old sorting principle (e.g. perseverance errors). More perseverative errors are considered as an outcome of worse shifting ability (Berg, 1948). Obesity has been associated with weaker shifting (Boeka \& Lokken, 2008; Fitzpatrick, Gilbert, \& Serpell, 2013; Perpiñá, Segura, \& Sánchez-Reales, 2016; Verdejo-García et al., 2010; Wu et al., 2014). Perseverating in unhealthy eating patterns such as overeating could be viewed as being stuck in unhealthy eating habits (Voon, 2015). Effective switching may facilitate goal pursuit, by allowing individuals to disengage from pursuing tempting short-term gratifications and pursue long-term self-regulatory goals instead (i.e., weight loss), and help individuals to switch strategy when means to reach these goals are suboptimal (Dohle, Diel, \& Hofmann, 2018).

\section{Time orientation}

An important EF-related construct is time orientation. The rationality of human behavior largely depends on the temporal frame adopted; behaviors that seem maladaptive in the long-run are usually driven by a strongly favorable balance of immediate costs and benefits (Hall \& Fong, 2007). Consuming high-caloric foods has the long-term costs of an elevated BMI and its associated health risks, but also provides the immediate benefit of satisfying hunger and the pleasurable experience of consuming a palatable, convenient snack. In contrast, many health enhancing behaviors such as the consumption of fruit and vegetables are associated with long-term benefits such as a healthy body weight and a lower risk of health diseases, but these foods are in the short term often considered as less palatable, more expensive and more time-consuming to prepare. To maintain or achieve a healthy weight and achieve accompanying health benefits in the future, individuals should show restraint in the present instead of indulging in unhealthy foods (Hall \& Fong, 2007). Time orientation is a broad concept and has been defined and conceptualized in many ways. The overarching idea is that individuals differ in the extent to which they in general focus on the present or future, and to what extent they consider present and future consequences of their current behavior. Individuals differ in their tendency to discount the future and making impulsive, short term, opportunistic 
choices (e.g. eating chocolate now rather than stick to a diet to lose weight) (Bickel \& Marsch, 2001). Two main conceptualizations of time orientation are Consideration of Future Consequences (CFC; Strathman, Gleicher, Boninger, \& Edwards, 1994) and delay discounting. Below, these two concepts are described in more detail and their possible association with obesity is explained.

\section{Consideration of Future Consequences}

The concept of Consideration of Future Consequences (CFC) is defined as the degree to which individuals consider the potential future outcomes of their present behaviors and to what extent they are influenced by these possible outcomes (Strathman et al., 1994). Whereas individuals scoring high on present time orientation tend to focus on the immediate consequences of their behavior, future-oriented individuals tend to give more importance to the future consequences of their current behavior (Strathman et al., 1994). CFC is measured with the 12-item CFC-scale (Strathman et al., 1994), of which Joireman, Shaffer, Balliet, and Strathman (2012) developed a 14-item version to improve the internal validity. Hereby they theoretically and empirically distinguished two subscales, CFC-immediate and CFC-future, instead of measuring CFC on a continuum as originally intended by Strathman et al. (1994). Research supports the best fit of a 2-factor solution for the CFC (Joireman, Balliet, Sprott, Spangenberg, \& Schultz, 2008; Joireman et al., 2012; Rappange, Brouwer, \& van Exel, 2009; Toepoel, 2010; van Beek, Antonides, \& Handgraaf, 2013). Thus, although scores on the CFC-future and CFC-immediate subscales are often negatively correlated, this does not necessarily have to be the case, meaning that people can have a strong focus on both the present and future. CFC has been associated with $\mathrm{BMI}$, and research provides some evidence that future-oriented individuals tend to have stronger intentions to eat healthy and a lower BMI, whereas present-oriented individuals tend to have weaker intentions to eat healthy and a higher BMI (Adams, 2012; Gick, 2014; Joireman et al., 2012).

\section{Delay discounting}

Individuals often choose a smaller, sooner reward because of a failure to value future events (Bickel, Jarmolowicz, Mueller, Gatchalian, \& McClure, 2012). This is captured in the concept of delay discounting, which is a key conceptualization of time orientation and generally used as a measure of impulsive choice. Delay discounting has also been seen as a measure of EF (e.g. Lamm, Zelazo, \& Lewis, 2006; Prencipe et al., 2011). It refers to the extent to which the value of a reinforcer decreases as a function of time (e.g. Mazur, 1987). Reinforcers lose their value quickly, leading to a preference for immediate rewards rather than long-term rewards (Chung \& Herrnstein, 1967). Reinforcement pathology as described in the CNDS theory is associated with steeper discounting, which 
means being more present-focused than future-oriented. It seems like later counts for less than now. Discounting is in essence adaptive because rewards only have benefits if they do occur, and this probability diminishes as the time until the reward extends. Choosing for a smaller, sooner reward instead of a larger, later reward is considered as impulsive choice behavior (Bickel et al., 2012). The shape of the human decision curve seems to be hyperbolic instead of exponential (Ainslie, 1975). An exponential curve would represent a rational choice pattern (e.g. the further away in time, the greater the drop in value). A consequence of hyperbolic discounting is that distinct preferences, which should always remain the same, may be reversed when one gets close to the time of gratification. This leads individuals to switch from a preference for a bigger, later reward to prefer a smaller, sooner reward, when the latter one becomes imminent. Thus, choices can change over time. Imagine you decided to skip dessert, but end up eating a piece of pie because it looked so delicious; maintaining a good figure seemed more important than eating pie until the palatable piece of pie appeared. Whereas a choice for immediate gratification is related to activity in the impulsive system, the choice for the delayed reward seems associated with activation in the executive system (McClure, Laibson, Loewenstein, \& Cohen, 2004). Delay discounting seems to bridge reflective and impulsive processes and may serve as a summary measure of the relative control by these two brain systems.

A common measure of delay discounting is the Monetary Choice Questionnaire (MCQ; Kirby, Petry, \& Bickel, 1999). In the MCQ participants are presented with hypothetical choices between smaller, immediate monetary rewards and larger, delayed monetary rewards. The discount rate of each participant can be estimated by calculating the k-value. $\mathrm{K}$ reflects the degree of discounting and thus can be seen as an 'impatience-factor' (Kirby \& Maraković, 1996). Individuals' discount rates have been found to be stable, at least over the short-term (Ohmura, Takahashi, Kitamura, \& Wehr, 2006). Delay discounting has been related to obesity, with a higher discount rate being related to an elevated BMI and unhealthy eating (Barlow, Reeves, McKee, Galea, \& Stuckler, 2016; Davis, Patte, Curtis, \& Reid, 2010; Epstein et al., 2014; Jarmolowicz et al., 2014; Kulendran et al., 2014; Weller, Cook III, Avsar, \& Cox, 2008). Results are however not consistent, as some studies failed to find this association (e.g. Nederkoorn, Smulders, Havermans, Roefs, \& Jansen, 2006; Rasmussen, Lawyer, \& Reilly, 2010), and it has been suggested that measuring delay discounting with food, which is a primary reinforcer, is more relevant for the association with obesity, than secondary reinforcers such as money (Rasmussen et al., 2010). 


\section{Targeting these components: cognitive control interventions}

It seems that to be able to make healthy food choices in the current obesogenic environment, effective self-regulation might help to avoid overeating. As described in the previous section, there are individual differences in both EF and EF-related constructs like time orientation that are found to be associated with variation in BMI. Specifically, an elevated BMI seems associated with weaker EF and a focus on present results and rewards (see Table 1 for an overview). If overeating is the result of diminished executive system control, then interventions that specifically engage and strengthen this system might restore the balance and thereby enhance self-control. Hence, one potential strategy to reduce overeating could be to strengthen cognitive control over consumption and body weight (Friese, Hofmann, \&Wiers, 2011). In this dissertation, we looked at different ways to support the reflective system by manipulating EF and time orientation, with the ultimate goal to strengthen cognitive control over eating behavior.

TABLE 1 Executive function (EF) and EF-related constructs included in this dissertation and their proposed role in food intake and weight loss.

\begin{tabular}{|c|c|c|c|}
\hline Construct & $\begin{array}{l}\text { Measurements included } \\
\text { in this dissertation }\end{array}$ & Definition & $\begin{array}{l}\text { Contribution to healthy } \\
\text { eating and weight loss }\end{array}$ \\
\hline Inhibition & $\begin{array}{l}\text { Go/No-Go task; Stop- } \\
\text { Signal Task, BRIEF-A - } \\
\text { inhibit subscale }\end{array}$ & $\begin{array}{l}\text { The ability to override } \\
\text { or interrupt undesired } \\
\text { behavioral responses. }\end{array}$ & $\begin{array}{l}\text { Resisting impulses aimed } \\
\text { at unhealthy food choices; } \\
\text { decreasing disinhibited } \\
\text { eating. }\end{array}$ \\
\hline Working memory & $\begin{array}{l}\text { 2-back task; BRIEF-A - } \\
\text { working memory subscale; } \\
\text { visuospatial working } \\
\text { memory task; backward } \\
\text { digit span task; object } \\
\text { memory task }\end{array}$ & $\begin{array}{l}\text { The ability to temporarily } \\
\text { store, monitor and } \\
\text { manipulate information. }\end{array}$ & $\begin{array}{l}\text { Planning meals; keeping } \\
\text { track of intake in mind; } \\
\text { keeping weight loss goals } \\
\text { in an active state. }\end{array}$ \\
\hline Shifting & $\begin{array}{l}\text { Wisconsin Card Sorting } \\
\text { Test; Trail Making Test; } \\
\text { BRIEF-A - shift subscale }\end{array}$ & $\begin{array}{l}\text { The ability to quickly } \\
\text { adjust to changing } \\
\text { demands and efficiently } \\
\text { switch between different } \\
\text { tasks. }\end{array}$ & $\begin{array}{l}\text { Switching to optimal } \\
\text { strategies to reach weight } \\
\text { loss goals depending on } \\
\text { the situation; overcoming } \\
\text { habits; applying flexible } \\
\text { dietary restraint. }\end{array}$ \\
\hline $\begin{array}{l}\text { Consideration } \\
\text { of Future } \\
\text { Consequences }\end{array}$ & $\begin{array}{l}\text { Consideration of Future } \\
\text { Consequences-general and } \\
\text { Consideration of Future } \\
\text { Consequences-food }\end{array}$ & $\begin{array}{l}\text { The degree to which } \\
\text { individuals consider the } \\
\text { potential future outcomes } \\
\text { of their present behaviors } \\
\text { and to what extent they } \\
\text { are influenced by these } \\
\text { possible outcomes. }\end{array}$ & $\begin{array}{l}\text { Considering the future } \\
\text { consequences of indulging } \\
\text { and the benefits of } \\
\text { restraint when making } \\
\text { food choices. }\end{array}$ \\
\hline Delay discounting & $\begin{array}{l}\text { Monetary Choice } \\
\text { Questionnaire }\end{array}$ & $\begin{array}{l}\text { The subjective loss of } \\
\text { value of a reinforcer as it } \\
\text { becomes more temporally } \\
\text { distant. }\end{array}$ & $\begin{array}{l}\text { Resisting immediate } \\
\text { reward of palatable } \\
\text { unhealthy foods in favor } \\
\text { of future weight loss and } \\
\text { health benefits. }\end{array}$ \\
\hline
\end{tabular}

BRIEF-A = Behavioral Rating Inventory of Executive Functioning - Adult version. 
EF has a strong genetic basis (Friedman et al., 2008), though it appears possible to improve performance of EF by extensive training (e.g. Hofmann et al., 2012; Karbach \& Verhaeghen, 2014). At the moment, research in the domain of eating behavior and EF training is scarce, thus empirical evidence for the (in)effectiveness of cognitive control training to support weight loss is lacking. As an exception, an extensive body of research on the training of inhibition has been conducted. Two meta-analyses showed a small but positive effect of training of inhibition on unhealthy food consumption: inhibition training reduces unhealthy food intake (Vanessa Allom, Mullan, \& Hagger, 2016; Jones et al., 2016). Training with the SST produced smaller effect sizes than GNG training, and behavior-specific GNG and SST training (e.g. with food stimuli) was more effective than training with neutral stimuli. It seems that training of general inhibition is not as effective at changing behavior as behavior-specific training, and it is not clear whether top-down inhibitory control is improved by these training paradigms. The effects on food intake seem moderated by the extent to which participants try to restrict food intake: current dieters or highly restrained eaters did profit the most from inhibition training (Jones et al., 2016). Thus, these results suggest that inhibition training can help to improve selfregulation and also change eating behavior, though the underlying mechanism is not clear yet.

Regarding working memory training, some first promising results addressing health behavior have been reported. For instance, working memory training reduced delay discounting in stimulant-abusers (Bickel, Yi, Landes, Hill, \& Baxter, 2011). More specifically, improvement in working memory seemed to generalize to less impulsive choice behavior and more valuation of future rewards. In a working memory training study with problem drinkers, participants reported reduced alcohol intake after the training, which was retained at one-month follow-up (Houben, Wiers, \& Jansen, 2011). A moderation effect with impulsivity towards alcohol cues was discovered, implying that the training of control processes (working memory) was the most effective for those with strong automatic processes, in line with the Reflective-Impulsive model (Hofmann, Friese, \& Strack, 2009; Strack \& Deutsch, 2004). Regarding shifting, little research has addressed possibilities to improve cognitive flexibility in the eating domain, though it seems possible to improve shifting via a wide variety of experimental training paradigms (Karbach \& Kray, 2009; Minear \& Shah, 2008; Zinke, Einert, Pfennig, \& Kliegel, 2012). Regarding time orientation, episodic future thinking (Atance \& O'Neill, 2001) seems a promising manipulation to alter delay discounting. We can travel in time in our mind and imagine future outcomes. This imaginative foresight seems crucial for human decision making, as this mental 'time travel' helps us to validate restrained choices now for benefits in the long term: it puts a so-called motivational 'brake' on impulsive choices (Boyer, 2008). Previous research has shown that episodic future thinking (i.e., imagining 
yourself in the future and 'pre-experience' an event), can reduce delay discounting (Benoit, Gilbert, \& Burgess, 2011; Lin \& Epstein, 2014; Peters \& Büchel, 2010), presenting episodic future thinking as a promising technique to alter time orientation in obesity. Thus, in general it seems possible to manipulate EF and time orientation.

\section{Aims and outline of this dissertation}

The main focus of this dissertation is on the role of EF and time orientation in eating behavior and weight loss. Previous results are mostly based on correlations with eating behavior and BMI, which does not provide insight in causality (e.g. Fitzpatrick et al., 2013). However, testing EF and EF-related constructs in experimental designs could shed light on proposed directions of causal associations. As the role and trainability of inhibition is already extensively studied, for the current dissertation, the focus is on the effectivity of manipulating the other core facets of EF, working memory and shifting. Also, the applicability of episodic future thinking in eating behavior will be studied. First, it is examined which cognitive components of EF and time orientation are associated with BMl, unhealthy eating behavior, and successful weight loss in a crosssectional and prospective design studying both individuals with obesity and a heathy weight community sample. Next, it is studied whether EF and time orientation can be manipulated or trained with the ultimate goal to improve healthy eating patterns and facilitate weight loss. To do so, we take a stepwise approach, studying the effectiveness of manipulation of each EF and time orientation in isolation. Before looking into interactions between factors, it is important that they are first identified and investigated in their own right. This dissertation is therefore divided in two main parts:

1. The Cognitive Profile of Obesity: Establishing the Association of Executive Functioning and Time Orientation with Eating Behavior and Weight Loss (chapters 2 and 3$)$.

2. Manipulating Cognitive Control: Episodic Future Thinking, Shifting and Working Memory Training (chapters 4, 5 and 6).

In chapter 2, it is examined whether better EF and less delay discounting are associated with weight loss success within individuals with obesity during multidisciplinary treatment, to confirm the role of these cognitive components in weight loss. To this end, first $\mathrm{EF}$ and delay discounting are examined in obese individuals who are at the start of their weight loss trajectory. Their performance on cognitive tasks and self-reported EF is compared with a sample of healthy weight controls. Next, it is analyzed whether better EF within the obese sample indeed predicts a greater change in BMI after six months of multidisciplinary treatment. In chapter $\mathbf{3}$, the role of time orientation will be studied in more detail in an online study conducted in a community sample. Both general and 
food-specific versions of measures of CFC and delay discounting are included, to find out whether food-specific versions of CFC and delay discounting are more predictive of eating behavior than the commonly used general measures. In the second part of this dissertation, small-scaled experimental studies are conducted to test the feasibility and preliminary effectiveness of new manipulations and training modules to strengthen cognitive control. Dependent variables of primary interest are improvements in EF and delay discounting, reduced food intake and weight loss. In chapter $\mathbf{4}$ the effectiveness of general and food-specific episodic future thinking in reducing delay discounting and food intake is studied. Female undergraduates are randomized to either an episodic future thinking or a control condition, and are asked to report either general or foodspecific events. In chapter $\mathbf{5}$, as research on the role and trainability of shifting is relatively scarce, a cognitive flexibility manipulation and its influence on eating behavior is tested in two studies. Paper-and-pencil exercises inspired by Cognitive Remediation Therapy (Tchanturia, 2014; Tchanturia, Davies, Reeder, \& Wykes, 2010) were offered to a female undergraduate sample. We examine whether shifting is amenable to manipulation, and whether this leads to changes in food intake. Study 1 is conducted to test the effectiveness of the cognitive flexibility manipulation. In study 2 , the manipulation is extended to two sessions and tested in a female undergraduate sample currently trying to restrain their food intake. Finally, in chapter $\mathbf{6}$ of this dissertation, we focus on the further development of working memory training, based on previous work conducted in our lab. In collaboration with the Instrumentation department of Maastricht University, a gamified version of the previously validated working memory training paradigm is developed. A game shell is put around the original paradigm to make it more fun and visually appealing to complete training sessions, and external rewards are added. As this previous working memory training did not result in a reduction of body weight (Houben, Dassen, \& Jansen, 2016), the working memory training is added to an online lifestyle treatment and is compared to a control condition who receives sham training combined with lifestyle treatment, instead of studying the effects of working memory training in isolation. In chapter $\mathbf{7}$, the main results of the studies included in this dissertation are summarized and discussed, along with key limitations and directions for future research. Finally, a summary of this dissertation is provided and opportunities for valorization are presented. 


\section{PART}

\section{The Cognitive Profile of Obesity}

Establishing the Association of Executive

Functioning and Time Orientation with

Eating Behavior and Weight Loss 



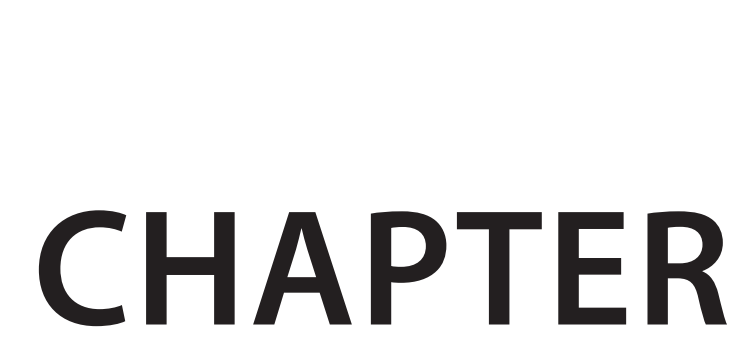

\section{Self-Regulation and Obesity:}

The Role of Executive Function and Delay Discounting in the Prediction of Weight Loss

In revised form accepted for publication as: Dassen, F. C. M., Houben, K., Allom, V., Jansen, A.

Self-regulation and obesity: The role of executive function and delay discounting in the prediction of weight loss (in press, Journal of Behavioral Medicine). 


\section{ABSTRACT}

Obesity rates are rising worldwide. Executive function and delay discounting have been hypothesized to play important roles in the self-regulation of behavior, and may explain variance in weight loss treatment success. First, we compared individuals with obesity ( $n=82)$ to healthy weight controls $(n=71)$ on behavioral and self-report measures of executive function (working memory, inhibition and shifting) and delay discounting. Secondly, the individuals with obesity took part in a multidisciplinary weight loss program and we examined whether executive function and delay discounting predicted weight change. Individuals with obesity displayed weaker general and food-specific inhibition, and weaker self-reported executive function. Better behavioral working memory and better self-reported inhibition skills in daily life were predictive of greater weight loss. As findings are correlational, future studies should investigate the causal relationship between executive function and weight loss, and test whether intervening on executive function will lead to better prevention and treatment of obesity.

Keywords:Weight Loss; Obesity; Body Mass Index; Executive Function; Delay Discounting 


\section{INTRODUCTION}

The worldwide prevalence of overweight and obesity is high with $37 \%$ of adults experiencing overweight or obesity ( $\mathrm{Ng}$ et al., 2014). Being overweight places individuals at risk of cardiovascular diseases, various forms of cancer, diabetes mellitus type II and musculoskeletal disorders (Lim et al., 2013). While there may be medical causes involved, most of the variance in Body Mass Index (BMI) can be attributed to behavioral factors (Ravussin \& Bogardus, 2000) that result in an energy imbalance (i.e., more energy is consumed than expended). Thus, to achieve weight loss, one needs to reduce caloric intake, increase physical activity, or do both. However, in practice, the prevention and treatment of obesity is not as straightforward. While many individuals may engage in weight loss attempts (De Ridder, Adriaanse, Evers, \& Verhoeven, 2014), about $80 \%$ of overweight dieters are not able to maintain their weight loss in the long run (Wing \& Phelan, 2005). Given the considerable health implications of obesity, it is important to find out what determines treatment success and successful weight loss.

Maintaining a healthy weight requires self-control (Hofmann, Friese, \& Roefs, 2009). According to dual-process theories of self-control a healthy lifestyle depends on the balance between two competing systems: the impulsive and the reflective system (Hofmann, Friese, \& Strack, 2009; Strack \& Deutsch, 2004). Behaviors relating to weight gain such as the overconsumption of palatable, energy-dense foods are the result of bottom-up impulses (i.e., the impulsive system) that are not sufficiently regulated via topdown cognitive control processes (i.e., the reflective system; Hofmann, Friese, \& Strack, 2009; Strack \& Deutsch, 2004). Executive function lies at the heart of cognitive self-control (Hofmann et al., 2012). Executive function is an umbrella term that refers to three main cognitivefunctions:(1)working memory-maintaining and updating relevantinformation; (2) inhibition - inhibiting prepotent impulses, and (3) shifting - rapidly and efficiently adapting to different situations (Miyake et al., 2000). Executive function allows for goaldirected action, and is important for planning and monitoring behavior, suppressing undesired responses, resisting temptations and creating alternatives, which are all highly relevant for engaging in and maintaining health behaviors, such as maintaining a diet in order to achieve weight loss (Dohle et al., 2018; Hall \& Marteau, 2014; Hofmann et al., 2012). For example, working memory is important for the regulation of food intake by keeping long-term goals active and down-regulating cravings for immediate desires (e.g. Hofmann et al., 2008) while inhibition assists in the suppression of automatic impulses to consume tasty, high-calorie foods (e.g. Guerrieri, Nederkoorn, \& Jansen, 2012; Hofmann, Friese, \& Roefs, 2009). Shifting has been hypothesized to be important in the selection of alternative means to pursue diet goals (e.g. Dohle et al., 2018; Hofmann et al., 2012), and has been found to be predictive of the extent to which individuals translate their healthy eating intentions into eating behavior (Allan, Johnston, \& Campbell, 2011). 
In line with dual-process accounts, weaker executive function appears to be related to elevated BMI (for reviews see Fitzpatrick et al., 2013; Prickett, Brennan, \& Stolwyk, 2015; Smith et al., 2011; Vainik, Dagher, Dubé, \& Fellows, 2013; Yang, Shields, Guo, \& Liu, 2018). However, the associations between BMI and each individual facet of executive function are not consistently found. Regarding inhibition, while it appears that the association between obesity and weaker inhibition is quite robust (for reviews see Bartholdy et al., 2016; Lavagnino et al., 2016), some studies only found differences in performance between individuals with obesity and healthy weight controls on a foodspecific inhibition task (e.g. Houben et al., 2014; Nederkoorn et al., 2012), and others found no differences in task performance (e.g. Hendrick, Luo, Zhang, \& Li, 2012; Loeber et al., 2012; though note that in the latter differences on self-report measures were significant). The association between working memory and BMI is also inconsistent. For example, some studies demonstrate a difference in working memory between individuals with obesity and those with a healthy weight (e.g. Maayan, Hoogendoorn, Sweat, \& Convit, 2011; Stingl et al., 2012), while other studies find no such difference (e.g. Ariza et al., 2012; Gonzales et al., 2010). Similarly, significant differences in shifting between individuals with obesity and those with a healthy weight have been found in a number of studies (e.g. Fagundo et al., 2012; Lokken, Boeka, Yellumahanthi, Wesley, \& Clements, 2010; Maayan et al., 2011), though not in all studies (Ariza et al., 2012; Mobbs, Iglesias, Golay, \& Van der Linden, 2011).

The inconsistency in these findings can perhaps be explained by the variability in the measures used to assess these constructs. There are many cognitive tasks reported to measure the domains of executive function (Miyake et al., 2000), and as a result, there is little consistency in methodology and results both within and across different domains of executive function. Thus, when studying executive function, it is imperative that a clear rationale for the selection of measures is presented (Etnier \& Chang, 2009). For the current study, representative and commonly used tasks were selected: the n-back task (Kirchner, 1958) to measure working memory, the Stop-Signal Task (Logan et al., 1997) to measure inhibition (general and food-specific), and the Trail Making Test (Reitan, 1958) to measure shifting. In addition to cognitive performance-based tasks, a self-report measure of executive function was also included, as both types of measures appear to capture different aspects of executive function: behavioral tasks seem to measure the efficiency of cognitive abilities while self-report measures seem more related to goal achievement in daily life (Toplak, West, \& Stanovich, 2013).

Most studies on the relationship between executive function and BMI are crosssectional in nature and examine differences between individuals of different weights. Examining whether executive function prospectively predicts weight gain and weight loss may be more useful in terms of weight loss intervention design. However, currently, 
few studies have examined the association between executive function and successful weight loss during a weight-loss intervention. Nederkoorn, Jansen, Mulkens, and Jansen (2007) showed that children with obesity who displayed weaker inhibition skills lost less weight during a multidisciplinary residential treatment for obesity. In line with this, Manasse et al. (2017) found that weaker general inhibition (though not food-specific inhibition) was associated with less weight loss during treatment. Hege et al. (2013) showed that differences in brain activity during a working memory task predicted successful weight loss during behavioral treatment, suggesting that the ability to encode or retrieve food and weight loss goals may contribute to the regulation of eating behavior. A more extensive prospective study, examining working memory, inhibition, shifting and planning, showed that poorer performance on a shifting task and more impulsive reactions on an inhibition task were associated with less weight loss after eight weeks (Galioto et al., 2016). This study included an extensive intervention program conducted over a short period of time using a relatively small sample. Weaker executive function has also been associated with less weight loss following bariatric surgery, possibly due to less adherence to post-operative guidelines for diet and physical activity (Spitznagel, Alosco, et al., 2013; Spitznagel, Garcia, et al., 2013). Thus, individual differences in executive function within individuals with obesity may explain why some individuals succeed during behavioral treatment and some do not. Currently, evidence for the role of executive function in the prediction of weight loss during treatment is insufficient.

Delay discounting is another executive function-related construct that could be important in weight regulation. Someone with a tendency to choose immediate over delayed rewards is considered to display greater delay discounting and therefore to be more impulsive (Ainslie, 1975). As described earlier, impulsivity or a lack of self-control is counteractive to the maintenance of a healthy weight. Individuals differ in their ability to delay gratification. Successful weight regulation requires that someone keeps his or her long term health in mind and does not always succumb to every tempting food stimuli. This tendency to discount future rewards has been related to obesity (for a review see Barlow et al., 2016), though note that this association has not been consistently found (Feda, Roemmich, Roberts, \& Epstein, 2015; Nederkoorn et al., 2006). Delay discounting has also been found to be predictive of subsequent weight gain in obese women (Kishinevsky et al., 2012). A preference for immediate rewards could reduce the success of an intervention, while the ability to delay gratification might facilitate successful weight loss and its maintenance. Evidence for the association between delay discounting and successful weight loss is limited. In a family-based obesity treatment, children who displayed high discounting and considered food to be highly reinforcing lost less weight (Best et al., 2012), whereas Manasse et al. (2017) did not find delay discounting to be predictive of weight loss during a standard behavioral treatment. 
Thus, evidence linking the specific facets of executive function and delay discounting to BMI and weight loss is inconsistent. Further, few studies have included all three facets of executive function in a single study. Therefore, the aim of the current study was twofold. First, we aimed to clarify the relationships between each facet of executive function and BMI, by comparing executive function performance, self-reported executive function, and delay discounting between individuals with obesity and healthy weight controls. We hypothesized that individuals with obesity would show weaker performance, and report lower scores, on executive function tasks and measures, and display more delay discounting, relative to the healthy weight controls. The second aim of this study was to examine whether executive function and delay discounting would predict successful weight loss following a multidisciplinary weight loss intervention. We hypothesized that individual differences in executive function and delay discounting among individuals with obesity at the start of a weight loss treatment would predict changes in BMI, such that better inhibition, working memory and shifting, and less delay discounting, would be associated with more weight loss controlling for weight at baseline. Determining whether baseline executive function or delay discounting predicts treatment success would provide additional targets for intervention.

\section{METHODS}

\section{Participants}

The sample of individuals with obesity were clients from an obesity treatment center who were about to start a multidisciplinary program targeting weight loss and lifestyle change ( $n=82,21$ male, mean age $=41.12 \pm 12.62$ years, age range $=18-71$ years). All clients who completed their intake session at the center and were eligible to start the weight loss intervention were either personally approached by a research assistant or received a flyer with information about the study. The test-session was scheduled within two weeks of the start of the weight loss intervention. Participants gave written permission for researchers to access their weight loss data at the weight loss center. The healthy weight controls were recruited from the general population via advertisements. They were matched on a group level to the individuals with obesity on age, gender and education level $(n=71,16$ male, mean age $=43.39 \pm 13.44$ years, age range $=18-69$ years). See Table 1 for an overview of baseline characteristics of the sample. Groups did not differ in age, gender or education level (all $p$ 's $>.28$ ), indicating that the matching procedure on a group level was successful. Individuals with obesity displayed, as expected, a higher BMI and a higher global Eating Disorder Examination-Questionnaire score, and they were strongly motivated to lose weight (all $p$ 's $<.001$ ). Six participants who completed the baseline measurement and were included in the first analysis, dropped 
out during the multidisciplinary treatment. As there is no weight loss data available for these participants, the final sample for the prediction of weight loss $(n=76)^{1,2}$ included $18(23.7 \%)$ males, with a mean age of $42.12(S D=12.47$, range $19-71)$ and a mean $\mathrm{BMI}$ of 39.19 ( $S D=5.35$, range $30.90-65.38$ ). The six participants who dropped out during treatment did not differ from the final sample on gender, education, motivation to lose weight or Eating Disorder Examination-Questionnaire score (all $p$ 's $>.13$ ), though they were significantly younger (drop-out: $M=28.50, S D=6.41 . p<.01$ ). The present study was approved by the local Ethical Review Commission.

TABLE 1 Characteristics of the sample displayed per weight group $(N=153)$.

\begin{tabular}{|c|c|c|c|c|}
\hline & $\begin{array}{l}\text { HWC }(n=71) \\
M(S D) / \%\end{array}$ & $\begin{array}{l}\text { OB }(n=82) \\
M(S D) / \%\end{array}$ & $\operatorname{tor} X^{2}(d f)^{a}$ & $p$ \\
\hline Age & $43.40(13.44)$ & $41.12(12.62)$ & $1.08(151)$ & .28 \\
\hline $\begin{array}{l}\text { Gender } \\
\text { Female }\end{array}$ & $77.5 \%$ & $74.4 \%$ & $0.06(1)$ & .80 \\
\hline $\begin{array}{l}\text { Education Level } \\
\text { Low } \\
\text { Medium } \\
\text { High }\end{array}$ & $\begin{array}{l}11.3 \% \\
64.8 \% \\
23.9 \%\end{array}$ & $\begin{array}{l}11.0 \% \\
69.5 \% \\
19.5 \%\end{array}$ & $0.48(2)$ & .79 \\
\hline BMI & $22.63(1.53)$ & $38.94(5.24)$ & $-26.90(96.65)$ & $<.001$ \\
\hline EDE-Q & $0.64(0.65)$ & $2.86(1.03)$ & $-16.17(138.76)$ & $<.001$ \\
\hline Motivation to lose weight & $1.97(0.91)$ & $4.48(0.49)$ & $-20.87(103.56)$ & $<.001$ \\
\hline
\end{tabular}

Note. $\mathrm{HWC}=$ Healthy weight controls; $\mathrm{OB}=$ individuals with obesity; $\mathrm{BMI}=$ Body Mass Index; $\mathrm{EDE}-\mathrm{Q}=$ Eating Disorder Examination - Questionnaire. ${ }^{a}$ Degrees of freedom vary across t-tests depending on violation of Levene's test for equality of variances; degrees of freedom were adjusted accordingly.

\section{The behavioral treatment program at the weight loss center}

The multidisciplinary treatment consisted of a 6 or 12-month program targeting weight loss and lifestyle change, tailored to the individual's needs and development. The program focused on physical activity (weekly group sport session and individual exercising at home) and nutritional advice to promote a healthy diet by a dietician, as well as counseling by a psychologist, based on principles of cognitive behavioral therapy.

\section{Materials and Measures}

\section{2-back task}

The n-back task was administered to measure behavioral working memory, based on the 2-back task used by Boselie, Vancleef, and Peters (2016). Stimuli (i.e., letters) were presented one-by-one on a computer screen, and participants had to indicate whether each letter was the same as the letter that was presented two stimuli ago by pushing 'yes' or 'no' on the keyboard. Within one trial, a single letter was presented on the screen 
for 500 milliseconds (ms), followed by a blank screen for 1500 ms. Participants started with a practice phase consisting of 30 trials, in which they received feedback after every response. Next, they completed the testing phase, consisting of 90 trials (30 target and 60 non-target letters). The main outcome variable was accuracy, which was the summation of correctly identified targets (hits) and non-targets (correct rejections). A higher accuracy displayed better behavioral working memory.

\section{Stop-Signal Task}

The Stop-Signal Task was used to measure behavioral inhibition (Logan et al., 1997). Both a general Stop-Signal Task (with $\mathrm{X}$ and $\mathrm{O}$ as go stimuli) and a food-specific Stop-Signal Task (with food pictures displayed in landscape or portrait orientation as go stimuli) were administered in counterbalanced order. Participants were instructed to respond as fast as possible to the go stimuli by pushing a left or right response key on the keyboard. However, if an auditory stop signal (beep) was presented, the participant had to inhibit his or her response and not push the key. During a go trial the letter or picture was presented for $1000 \mathrm{~ms}$, preceded by a $500 \mathrm{~ms}$ fixation point. The delay between the go signal and the stop signal (go-stop delay) started at $250 \mathrm{~ms}$ and was subsequently adjusted by a tracking procedure. If the participant successfully inhibited the response, the go-stop delay was increased by $50 \mathrm{~ms}$, and if the participant was not able to inhibit the response, the delay decreased by $50 \mathrm{~ms}$. The dependent variable was the stop signal reaction time, which was calculated by subtracting the mean stop delay from the mean reaction time. A higher stop signal reaction time is indicative of less inhibitory control. Percentage of inhibited trials was verified afterwards. If a participant never stopped at a stop signal, and thus failed to comply with the instructions, no stop signal reaction time could be calculated.

\section{Trail Making Test}

The Trail Making Test was administered to assess behavioral shifting (Reitan, 1992). The Trail Making Test consists of two parts (part A and part B). In part A, participants had to connect 25 circles numbered from 1 to 25 as fast as possible. In part B, the participants had to connect 24 circles numbered from 1 to 12 with letters from $A$ to $L$ in alternating order (e.g. 1-A-2-B-3-C etc.) as fast as possible. When an error was made, the participant was instructed to return to the place where the error originated and to continue from there. The outcome variable was the difference score, which was the time to complete part B minus time to complete part A (B-A). This outcome measure provides a relatively pure indicator of shifting ability, as it minimizes visuoperceptual and working memory demands (Sanchez-Cubillo et al., 2009). The higher the difference score, the poorer the shifting ability. 


\section{Monetary Choice Questionnaire}

Participants completed the 27-item Monetary Choice Questionnaire (Kirby et al., 1999) which is a common measure of delay discounting. They were presented with 27 hypothetical choices between smaller, immediate monetary rewards and larger, delayed monetary rewards (Kirby et al., 1999). Immediate rewards ranged from $€ 11-78$ and delayed rewards ranged from $€ 25-85$, with a delay of 7-186 days. An example choice is 'Would you prefer $€ 55$ today, or $€ 75$ in 61 days?' Participants' hyperbolic discount parameter ( $k$ ) was determined as described by Kirby et al. (1999). The geometric mean of the k-value was used as outcome variable (Kaplan, Lemley, Reed, and Jarmolowicz, 2014). Values of $k$ could range from 0.00016 to 0.25 , with higher values indicating a preference for smaller, immediate rewards over larger, delayed rewards, thus a higher level of discounting. Because raw k-values tend to be skewed, k-values were normalized using a log transformation.

\section{Behavioral Rating Inventory of Executive Functioning - Adult version}

The Behavioral Rating Inventory of Executive Functioning - Adult version (Roth, Isquith, \& Gioia, 2005) is a 75-item standardized rating scale developed to provide a window into everyday behaviors associated with specific domains of executive functioning in adults. The Behavioral Rating Inventory of Executive Functioning consists of nine subscales, of which we used the three subscales which correspond to the three facets of executive function targeted in the current study: the working memory, inhibit, and shift subscales. Participants had to indicate for each item whether the statement applied to them on a 3-point Likert scale, with the options 'never,', 'sometimes' or 'always'. Example item are: 'I tap my fingers or bounce my legs' (inhibit), 'I have trouble concentrating on tasks (such as chores, reading, or work)' (working memory), 'I have trouble changing from one activity or task to another' (shift). The raw scale scores were transformed to T-scores (standardized scores with $M=50$ and $S D=10, T \geq 65=$ clinically significant, Roth et al., 2005) with higher T-values reflecting weaker executive function.

\section{Eating Disorder Examination - Questionnaire 6.0}

The Eating Disorder Examination-Questionnaire 6.0 (Fairburn \& Beglin, 2008) is a 28-item self-report questionnaire that assesses disordered eating behaviors and attitudes over the previous 28 days, and was assessed to characterize the sample. Each item is scored on a 7-point scale indicating the frequency or severity of the item. The items are scored across four subscales: restraint, weight concerns, shape concerns, and eating concerns, which are combined into one global score with higher scores reflecting greater levels of eating psychopathology. 


\section{Motivation to lose weight}

Participants indicated on a 5-point Likert scale the extent of importance of four statements regarding motivation to lose weight, ranging from (1) 'totally not important' to (5) 'extremely important'). An example of an item is: 'How important is it for you to lose weight?'The four items showed good reliability, with a Cronbach's alpha of .96, and therefore a total score was calculated by summing up the scores of the individual items and taking the average, with a higher score indicating a stronger motivation to lose weight.

\section{Percentage BMI loss (e.g. BMI change)}

BMI data of individuals with obesity at baseline and after six months of treatment were provided by the obesity treatment center. Participants were weighed and measured while wearing their clothes but without shoes. BMI was calculated with the formula: (height in $\mathrm{cm}$ ) / (weight in $\mathrm{kg})^{2}$. To correct for initial differences in BMI between participants, change in BMI was calculated as a percentage of BMI loss relative to baseline BMI (Deitel \& Greenstein, 2003). Percentage BMI loss was calculated with the formula: ((BMI baseline - BMI post-measurement) / BMI baseline) $x 100$.

\section{Procedure}

Participants completed one individual session in which executive function and delay discounting were assessed via behavioral tasks and questionnaires. Individuals with obesity were tested at the obesity treatment center in a private room, whereas healthy weight controls were tested at the university. First, participants performed the behavioral tasks to measure executive function performance (2-back task, Trail Making Test, Stop-Signal Task-general and Stop-Signal Task-food). The behavioral tasks were administered in randomized order. Next, participants completed the Monetary Choice Questionnaire to measure delay discounting, the Behavioral Rating Inventory of Executive Functioning - Adult version, Eating Disorder Examination-Questionnaire, motivation to lose weight and demographics. Finally, healthy weight controls were measured and weighed at the university. Individuals with obesity were measured and weighed at the obesity treatment center at the start of their weight loss intervention and again six months later. All participants received a voucher of $€ 10$ as compensation for participation. 


\section{Statistical analyses}

First, the data were inspected for outliers (i.e., mean \pm 3 standard deviations). One outlier was observed for the Trail Making Test (healthy weight controls), and two outliers were observed for the Stop-Signal Task-general (individuals with obesity). These outliers were observed above the mean and were therefore adjusted to the highest value that was not considered an outlier plus one for each measure. To investigate the association between behavioral tasks and questionnaires, Pearson correlation coefficients were calculated. Next, individuals with obesity at baseline and healthy weight controls were compared on their performance on the executive function behavioral tasks and selfreport subscales using MANOVA's testing for group differences in 1) working memory, general and food-specific inhibition, shifting and delay discounting, and 2) the working memory, inhibit and shift subscales of the Behavioral Rating Inventory of Executive Functioning (self-report). Significant effects were further analyzed using Bonferroni corrected univariate statistics. Further, hierarchical regression analyses were conducted with percentage BMI loss as outcome variable. Age, gender and education level were entered in step one. First we looked at the predictive effect of each measure by entering them individually into the model. Next, given that there was no a-priori hypothesis about which factor would be most predictive, all predictors were entered simultaneously in step two. Subsequently, a backward regression was performed to test for a final model including all significant predictors.

\section{RESULTS}

\section{Descriptives}

See Table 2 for an overview of correlations between predictors. Behavioral working memory correlated moderately with all other predictors, indicating that better performance on the 2-back task was related to better performance on the other executive function tasks, less delay discounting and better self-reported executive function. The general and food-specific Stop-Signal Task displayed a strong positive correlation, indicating that both measures capture different, though related facets of behavioral inhibition. The behavioral measure of shifting was positively associated with self-reported shifting, though note that the correlation with self-reported working memory and inhibition was also significant and comparable to this result. Self-reported inhibition correlated with food-specific but not general behavioral inhibition. The Behavioral Rating Inventory of Executive Functioning subscales were moderate to strong positively correlated with each other. 


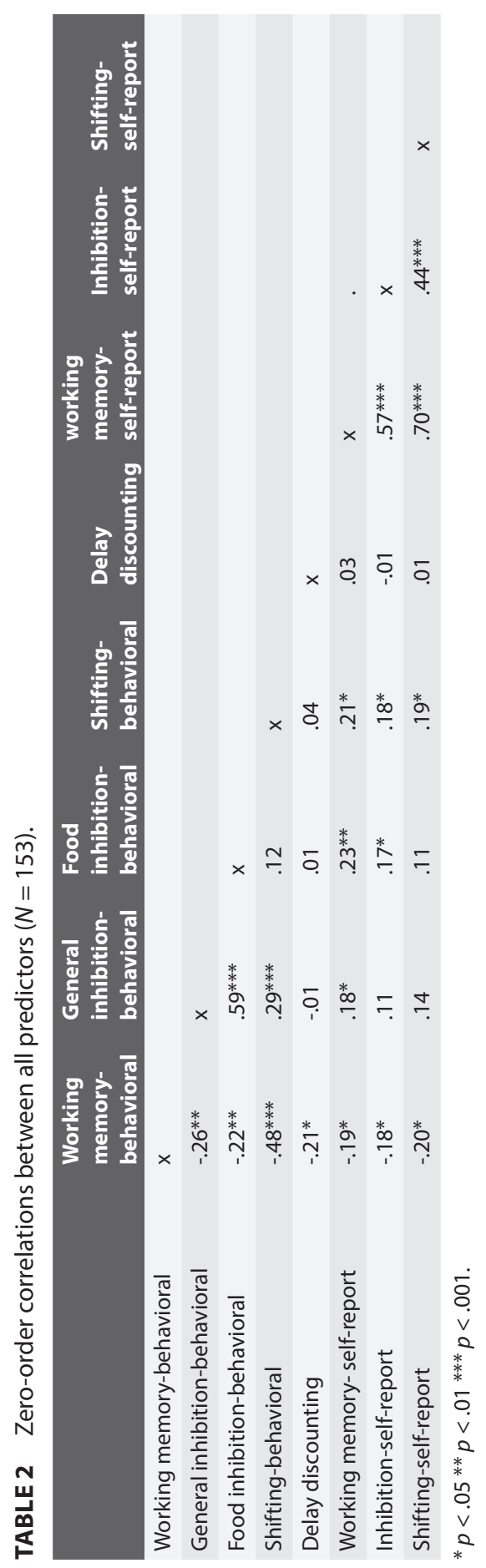




\section{Comparing executive function between individuals with obesity and healthy weight controls}

A one-way MANOVA was conducted to investigate group differences in executive function and delay discounting. Five dependent variables were included in the first MANOVA: behavioral working memory, behavioral shifting, general behavioral inhibition and food-specific behavioral inhibition and delay discounting ${ }^{1,2}$. Overall, the MANOVA revealed a significant difference between groups, $F(5,139)=3.73, p<.01, \eta_{p}^{2}=.12$. Post-hoc analyses using a Bonferroni adjusted alpha level of .01, revealed significant differences between groups for both inhibitory control outcomes: general behavioral inhibition, $F(1,143)=13.18, p<.001, \eta_{p}^{2}=.08$, and food-specific behavioral inhibition, $F$ $(1,143)=10.30, p<.01 \eta_{p}^{2}=.07$. Inspection of the mean scores indicated that individuals with obesity displayed a higher stop signal reaction time (i.e., less behavioral inhibition) on the general and food-specific Stop-Signal Task than healthy weight controls (see Table 3 for an overview of all means and standard deviations per group).

A MANOVA including the three subscales of the Behavioral Rating Inventory of Executive Functioning (working memory, inhibit and shift) was performed to investigate group differences in self-reported executive function. Overall, the MANOVA revealed a significant difference between groups, $F(3,149)=3.61, p=.015, \eta^{2}{ }_{p}=.07$. Post-hoc analyses, using a Bonferroni adjusted alpha level of .017, indicated that there were significant differences between groups on both self-reported working memory and inhibition: working memory, $F(1,151)=9.70, p=.002, \eta_{p}^{2}=.06$, and inhibition, $F(1,151)=6.40, p=.012, \eta_{p}^{2}=.04$. Results for shifting did not reach significance, $F(1$, $151)=3.30, p=.071, \eta_{p}^{2}=.02$. Inspection of the mean scores indicated that individuals with obesity reported higher T-values (e.g. weaker executive function) on the working memory, inhibition and shifting subscales (see Table 3).

TABLE 3 Univariate follow-up analyses of comparison between weight groups, means with standard deviations in parentheses are displayed.

\begin{tabular}{lllll} 
Predictors & HWC $(\boldsymbol{n}=\mathbf{7 1})$ & $\begin{array}{l}\text { OB }(\boldsymbol{n}=\mathbf{8 2}) \\
\boldsymbol{M}(\boldsymbol{S D})\end{array}$ & $\boldsymbol{F}(\boldsymbol{d f f})^{\mathrm{a}}$ & $\boldsymbol{p}$ \\
\hline Working memory-behavioral & $72.30(14.57)$ & $72.83(11.45)$ & $0.06(1,143)$ & .81 \\
General inhibition-behavioral & $287.76(50.15)$ & $319.66(55.29)$ & $13.18(1,143)$ & $<.001$ \\
Food inhibition-behavioral & $329.39(61.26)$ & $347.51(69.55)$ & $10.30(1,143)$ & $<.01$ \\
Shifting-behavioral & $23.77(14.81)$ & $26.29(14.45)$ & $0.24(1,143)$ & .30 \\
Delay discounting & $-4.77(1.83)$ & $-4.62(1.87)$ & $1.08(1,143)$ & .63 \\
Working memory-self report & $53.52(10.17)$ & $59.35(12.63)$ & $9.70(1,151)$ & $<.001$ \\
Inhibition-self report & $51.01(9.82)$ & $55.22(10.62)$ & $6.40(1,151)$ & .01 \\
Shifting-self-report & $52.66(9.09)$ & $55.71(11.32)$ & $3.30(1,151)$ & .07
\end{tabular}

Note. $\mathrm{HWC}=$ Healthy weight controls; $\mathrm{OB}=$ individuals with obesity

a Degrees of freedom vary due to missing data at random, as MANOVA applies listwise deletion. 


\section{Executive function as predictor of BMI change}

Participants lost on average $2.95 \mathrm{BMI}$ points $(S D=1.98)$, though results ranged from an increase of 0.40 to a decrease of $10 \mathrm{BMI}$ points. Regarding percentage BMI loss, participants lost on average $7.22 \%$ of their baseline BMI $(S D=4.80 \%)$, ranging from an increase of $1.16 \%$ in BMI to a decrease of $23.50 \%$. In the hierarchical regression analysis predicting percentage BMI loss, demographics (age, gender and education level) were entered in step 1 , and each predictor was entered individually at step 2 . Results revealed behavioral working memory to be a significant predictor of $\mathrm{BMI}$ change $(\beta=.31, p=$ .03 ), and a marginally significant contribution of self-reported inhibition was found ( $\beta$ $=-.23, p=.051$ ). None of the other predictors reached significance (all $p>.39$ ). Next, all executive function outcomes and delay discounting were entered at once in step 2 . Demographics predicted a total of $7.5 \%$ of variance in percentage BMI loss, and adding all predictors in step two accounted for an additional $19.5 \%$ of variance. Table 4 shows the results of the hierarchical model including all predictors. The model as a whole was marginally significant, $F(12,58)=1.79, p=.07$. Significant predictors in step 2 , besides gender and education, were self-reported inhibition and behavioral working memory.

TABLE 4 Results of hierarchical linear regression analyses predicting percentage Body Mass Index loss after six months of multidisciplinary treatment $(n=76)$.

\begin{tabular}{|c|c|c|c|c|c|}
\hline Fixed effect & B & SE B & $\boldsymbol{\beta}$ & $t$ & $p$ \\
\hline \multicolumn{6}{|l|}{ Step 1} \\
\hline Constant & 9.77 & 2.74 & & & \\
\hline Age & 0.00 & 0.05 & .01 & 0.05 & .96 \\
\hline Gender & -2.47 & 1.36 & -.22 & -1.82 & .07 \\
\hline Medium education & -0.47 & 1.79 & -.05 & -0.26 & .80 \\
\hline High education & -2.07 & 2.07 & -.18 & -1.00 & .32 \\
\hline \multicolumn{6}{|l|}{ Step 2} \\
\hline Constant & -0.43 & 7.97 & & & \\
\hline Age & -0.02 & 0.05 & -.06 & -0.43 & .67 \\
\hline Gender & -3.59 & 1.53 & -.32 & -2.50 & .02 \\
\hline Medium Education & -3.76 & 2.02 & -.37 & -1.86 & .07 \\
\hline High Education & -5.23 & 2.48 & -.45 & -2.11 & .04 \\
\hline Working memory-behavioral & 0.16 & 0.07 & .39 & 2.50 & .02 \\
\hline General inhibition-behavioral & -0.00 & 0.01 & -.02 & -0.14 & .89 \\
\hline Food inhibition-behavioral & 0.01 & 0.01 & .07 & 0.47 & .64 \\
\hline Shifting-behavioral & 0.03 & 0.05 & .08 & 0.54 & .59 \\
\hline Delay discounting & -0.36 & 0.32 & -.14 & -1.13 & .27 \\
\hline Working memory-self-report & 0.07 & 0.07 & .19 & 1.04 & .30 \\
\hline Inhibition-self-report & -0.17 & 0.06 & -.37 & -2.65 & .01 \\
\hline Shifting-self-report & 0.08 & 0.08 & .18 & 0.97 & .34 \\
\hline
\end{tabular}

Note. $R^{2}=.08$ for Step $1, \Delta R^{2}=.20$ for Step $2(p=.07)$. 
Finally, we conducted a backward regression method to find the best model by excluding non-significant predictors. The final model reached significance, $F(6,64)=$ $3.14, p<.01$.The total variance explained by the final model was $22.8 \%$ and included gender $(\beta=-.32, p<.01)$ and education level $(\beta=-.33, p=.08$; and $\beta=-.43, p=.03$ for medium and high education level respectively), behavioral working memory $(\beta=.33$, $p=.02)$ and both the inhibit $(\beta=-.30, p=.02)$ and shift subscale $(\beta=.28, p=.04)$. This indicated that males and participants with a lower education lost more weight. Besides demographics, behavioral working memory was the strongest predictor of weight loss, followed by self-reported better inhibition in daily life, indicating that executive function explains a significant part of variance of percentage BMI loss. The shift subscale also contributed significantly to the final model, with less shifting being related to more weight loss, which was contrary to our hypothesis (though note that the $\beta$ was neither significant when entered individually in the prediction, $\beta=.08, p=.63$, nor in the first model including all predictors; see Table 4).

\section{DISCUSSION}

The main aim of this study was twofold: 1) to examine whether individuals with obesity would show weaker performance on executive function measures and display more delay discounting relative to healthy weight controls, matched (group level) on age, gender and education level, and 2) whether executive function and delay discounting would be predictive of weight loss during a subsequent multidisciplinary weight loss treatment. This study included measures of the three main facets of executive function (Diamond, 2013; Miyake et al., 2000) and delay discounting in one study. Results show that individuals with obesity displayed less efficient inhibition on the Stop-Signal Task, both general and food-specific, relative to healthy weight controls and that they reported weaker executive function on the subscales of the Behavioral Rating Inventory of Executive Functioning. No differences on the 2-back task or Trail Making Test were found between groups, thus, individuals with obesity did not display weaker behavioral working memory or shifting performance than healthy weight controls. Regarding the prediction of weight loss, behavioral working memory was the strongest predictor of change in BMI besides gender and education level. In addition, more difficulties in daily life with respect to inhibition as indicated on the inhibit subscale of the Behavioral Rating Inventory of Executive Functioning, and less shifting as indicated on the shift subscale of the Behavioral Rating Inventory of Executive Functioning also contributed significantly to the prediction of BMI loss.

Performance on both the general and food-specific Stop-Signal Task differed between individuals with obesity and healthy weight controls, with individuals with obesity displaying less efficient inhibition. These results are consistent with those of 
Bartholdy et al. (2016); however, they differ from Houben et al. (2014), who only found an association between elevated BMI and weaker performance on a food-specific StopSignal Task. The inconsistency in findings may be explained by the different samples used in each study. The current sample included individuals with obesity rather than predominantly individuals who were overweight. It may be the case that differences in general inhibition are only apparent at the higher end of the weight spectrum. Previous research did show promising results of food-specific inhibition training on food intake and weight loss (Houben \& Jansen, 2015; Lawrence et al., 2015), whereas studies in which general inhibition was targeted were not successful (Allom, Mullan, et al., 2016).

Individuals with obesity reported more problems experienced in daily life as indicated on the subscales of the Behavioral Rating Inventory of Executive Functioning (though note that the shift subscale did not reach significance), in line with our hypothesis. While working memory was reported as weaker in individuals with obesity, behavioral working memory as measured with the 2-back task did not differ between individuals with obesity and healthy weight controls. Behavioral tasks and ratings of executive functioning have been suggested to reflect different underlying constructs (Allom, Panetta, Mullan, \& Hagger, 2016; Toplak et al., 2013). Thus, based on the current results, obesity is not associated with working memory and shifting as measured by behavioral tasks, though individuals with obesity do seem to experience less successful goal pursuit in daily life.

The current results indicate that inhibition and self-reported executive function are important in preventing obesity, as they appear to be a risk factor for weight gain (e.g. Dohle et al., 2018; Hofmann et al., 2008). However, given that the current study was crosssectional in nature it is uncertain whether these factors indeed cause obesity, or obesity causes poorer executive function, or whether there is another factor explaining both. There are indications that impaired executive function leads to impaired self-regulation (e.g. higher food intake, less exercise), rendering individuals with deficient executive function more predisposed to becoming obese (Dohle et al., 2018; Hofmann et al., 2012). However, there is also evidence that obesity leads to impaired cognitive functioning via reduced blood flow to the areas of the brain that control executive function or abnormalities in glucose and insulin regulation (Boeka \& Lokken, 2008; Smith et al., 2011). Conversely, the results of a recent meta-analysis indicated a significant positive effect of weight loss on executive function (Veronese et al., 2017). This suggests that the association between executive function and obesity may be bidirectional (Kanoski \& Davidson, 2011; Sellbom \& Gunstad, 2012). To shed more light on this bidirectional relationship, future research should study these factors in prospective designs including pre- and posttests or experimental designs including a manipulation of executive function. 
Rather than focusing on weight gain, the present study focused on examining the prediction of weight loss. Accuracy at the 2-back task was the strongest predictor of a decreased BMI after treatment, suggesting an important role for working memory in weight loss success. Specifically, working memory may help to keep weight loss goals in an active state, so individuals can efficiently monitor whether their food intake is in line with their weight loss goals (Boutelle \& Kirschenbaum, 1998). This is in line with dual process models that suggest that the relative influence of each system on selfregulation differs as a function of working memory such that individuals with better working memory display more goal-directed behavior (e.g. Hofmann et al., 2008). Our results are in line with Hege et al. (2013), who also showed the predictive value of working memory for successful weight loss during a lifestyle treatment. Hege et al. (2013), however, used a food-specific working memory task, which could be even more predictive in the self-regulation of weight loss given the role of working memory in food cue monitoring (Meule, 2016). Based on these results, targeting weaker working memory seems a promising target for intervention (Jansen et al., 2015). Preliminary results of working memory training indicate promising short-term effects of training on food intake, though long-term effects on weight loss have not yet been established (Dassen, Houben, Van Breukelen, \& Jansen, 2018; Houben et al., 2016).

The inhibit subscale was also a significant predictor of weight loss, with participants who reported less efficient inhibition in daily life displaying a smaller BMI change. The Stop-Signal Task, in contrast, was not related to weight loss. This is contrary to our hypothesis and not in line with previous research (e.g. Galioto et al., 2016; Manasse et al., 2017; Nederkoorn et al., 2007). Shifting as measured with the Trail Making Test did not differ between individuals with obesity and healthy weight controls, and was also not predictive of subsequent weight loss. Importantly, many executive function tasks were originally developed as indicators of brain damage, including the Trail Making Test (Reitan, 1958). Therefore, it is possible that this task was not sensitive enough to pick up subtle impairments in shifting ability (Fitzpatrick et al., 2013). Task impurity is also a common problem in this field of research, as tasks may be thought to measure a particular facet of executive function but the execution of any task may also involve diverse cognitive systems in addition to the targeted facet (Jurado \& Rosselli, 2007). Surprisingly, the direction of the association of the shift subscale with weight loss was in the opposite direction than expected in the backward regression, with less shifting ability being predictive of more weight loss. The role of shifting in eating behavior is relatively unexplored (Dohle et al., 2018). Shifting ability may be beneficial to weight loss, helping individuals to switch strategy when the current strategy is suboptimal, though this ability could also allow individuals to easily switch from their weight loss goal to more tempting immediate options (Hofmann et al., 2012). However, note that in 
our study, the contribution of the shifting subscale was small and the correlation with change in BMI was not significant, warranting cautious interpretation.

Delay discounting did not differ between individuals with obesity and healthy weight controls, and also did not add significantly to the prediction of weight loss, though results were in the expected direction. In the current study, we measured delay discounting with the Monetary Choice Questionnaire, which uses money as a reinforcer. However, it has been suggested that a food-specific delay discounting task would be more predictive of BMI and weight loss (e.g. Rasmussen et al., 2010). Current results provide no evidence for a direct relationship of delay discounting with weight loss. Importantly, we followed participants until six months after the start of their weight loss intervention. As weight regain is one of the biggest problems in weight loss treatment (Ikeda et al., 2005), it would be interesting to continue to monitor participants after finishing the treatment, to examine the potential role of executive function and delay discounting in the maintenance of weight loss (Gettens \& Gorin, 2017). It has been suggested that self-regulation becomes even more important when the intensive treatment ends, as the external regulation coming from the treatment program also ends at this point (Halberstadt et al., 2013).

In conclusion, executive function appears to be weaker in individuals with obesity, in line with the results of a recently published meta-analysis (Yang et al., 2018). We discovered weaknesses on self-report measures and both general and food-specific inhibition. Executive function is also predictive of weight loss, with especially working memory and self-reported inhibition being associated with a greater decrease in BMI following treatment. A significant contribution of behavioral shifting or delay discounting to the prediction of weight loss could not be established. Based on current results, it would be interesting for future studies to explore whether adding a cognitive screening at the start of a weight loss trajectory could be an objective way to identify individuals who might benefit from additional executive function training to facilitate optimal weight. These results suggest new avenues for the improvement of current behavioral treatments, and may increase future treatment success. 


\section{Compliance with Ethical Standards Conflict of Interest}

All authors declare that they have no conflicts of interest.

\section{Ethical approval}

All procedures performed in studies involving human participants were in accordance with the ethical standards of the institutional and/or national research committee and with the 1964 Helsinki declaration and its later amendments or comparable ethical standards.

\section{Informed consent}

Informed consent was obtained from all individual participants included in the study.

\section{ACKNOWLEDGEMENTS}

This project was funded by the Maastricht University Interfaculty Program 'Eatwell'. We are grateful to CO-EUR Hoensbroek and CO-EUR Maastricht for providing us with the opportunity to conduct our research at their treatment centers. We especially want to thank Dr. Erik Aller for giving permission to conduct our study at CO-EUR, and Daisy Jeurissen and the members of the secretary for their assistance in the recruitment of the individuals with obesity and for providing us with the weight loss data. We want to thank Dennis Ruesink, Kyra Wijnen, Hajar Riane and Manon Warnier for their assistance with the data collection.

\section{Footnotes}

1 Two data points (individuals with obesity) and one additional value (healthy weight controls) were excluded from the general Stop-Signal Task because the percentage of correct stop trials was zero, indicating that these participants never inhibited their response. Two additional data points (individuals with obesity) were excluded from the food-specific Stop-Signal Task because the percentage of correct stop trials was zero.

2 One participant was excluded from all behavioral tasks because of a physical disability affecting reaction times (individuals with obesity); one participant was excluded from the Trail Making Test because of wrong execution of instructions (individuals with obesity); data is missing for one participants for both the general and food-specific Stop-Signal Task because of technical problems in data storage (individuals with obesity); data is missing for one participant on the 2-back task because the participant did not correctly understand the instructions (individuals with obesity) 



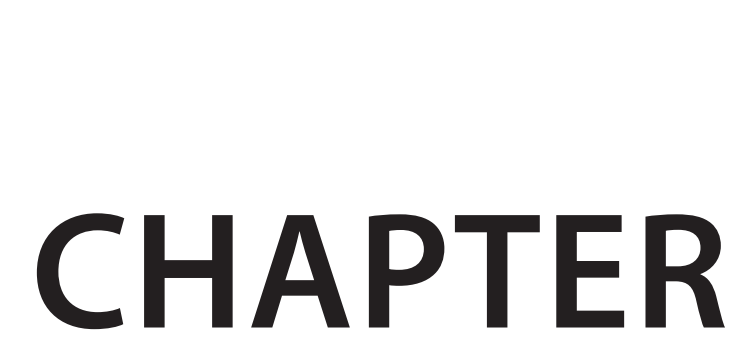

Time Orientation and Eating Behavior: Unhealthy Eaters Consider Immediate Consequences, while Healthy Eaters Focus on Future Health

Published as: Dassen, F. C. M., Houben, K., Jansen, A. (2015).

Time orientation and eating behavior: unhealthy eaters consider immediate consequences, while healthy eaters focus on future health. Appetite, 91, 13-19. https://doi. org/10.1016/j.appet.2015.03.020 


\section{ABSTRACT}

Time orientation could play an important role in eating behavior. The current study investigated whether eating behavior is associated with the Consideration of Future Consequences scale (CFC). Specifically, it was examined whether unhealthy eaters consider the future less and are more concerned with immediate gratification. A related measure of time orientation is delay discounting, a process by which a reinforcer becomes less valuable when considered later in time. Recent research argues that the relation between time orientation and health behaviors is measured best at a behaviorspecific level. In the current study, we explored the relationships between CFC and discount rate - both general and food-specific - and their influence on healthy eating. Participants ages 18 to $60(N=152$; final sample $N=146)$ filled in an online questionnaire consisting of the CFC, a food-specific version of the CFC (CFC-food), the Monetary Choice Questionnaire (MCQ) and an adapted MCQ version with snack food as a reinforcer. Self-reported healthy eating was positively related to the future subscale $(r=.48, p<$ $.001)$ and negatively to the immediate subscale of the CFC-food $(r=-.43, p<.001)$. The general CFC and discount rate (MCQ and MCQ-snack) were not related to healthy eating (all $p>.05$ ). In order to predict behavior, measurements of time orientation should thus be tailored to the behavior of interest. Based on current results, shifting one's concern from the immediate consequences of eating to a more future-oriented perspective may present an interesting target for future interventions aimed at promoting healthy eating and reducing overweight.

Keywords: Time Orientation; Consideration of Future Consequences; Delay Discounting; Behavior-Specific Measurements; Healthy Eating 


\section{INTRODUCTION}

Future-oriented thinking could be conducive to a healthier lifestyle. Adopting a healthy lifestyle poses a conflict between the short-term and long-term benefits of a person's actions (Joireman et al., 2012). Consuming highly palatable unhealthy foods has immediate pleasurable results, whereas healthy eating may be less immediately rewarding, but offers benefits in the long run. An important source of self-control failures could be a tendency to give more importance to these short-term implications (e.g. taste, satiation), relative to long-term implications (e.g. health and body weight) (Liberman \& Trope, 2008). Shifting the temporal focus away from these immediate benefits towards the future outcomes seems necessary in order to make healthy choices (Hall \& Fong, 2007). The concept of Consideration of Future Consequences (CFC) attempts to measure differences in the degree to which individuals consider the potential distant outcomes of their current behaviors and to what extent they are influenced by these potential outcomes (Strathman et al., 1994). Present-oriented individuals tend to focus on the immediate consequences of their behavior, whereas future-oriented individuals give more importance to the future consequences, even if there are immediate costs (Strathman et al., 1994).

CFC has been linked to many health behaviors. Studies indicate that individuals with a future orientation are more likely to quit smoking (Kovač \& Rise, 2007), show more dietary control (Piko \& Brassai, 2009) and are more likely to exercise (Adams \& Nettle, 2009). According to recent research, CFC-immediate and CFC-future are empirically distinguishable predictors, instead of CFC being seen as one continuum (Adams, 2012; Arnocky, Milfont, \& Nicol, 2013; Joireman et al., 2008; Joireman et al., 2012; Petrocelli, 2003; Rappange et al., 2009; Toepoel, 2010). Specifically, the CFC-immediate subscale comprises concern with immediate, short-term consequences of behavior (e.g. satisfying immediate concerns or needs) while the CFC-future subscale comprises concern with future, long-term consequences of behavior (e.g. achieving future outcomes or goals). Both subscales seem to relate differently to behavior. For example, Joireman et al. (2012) found healthy eating and exercising to be related only to the future subscale, which was in line with a promotion orientation in which individuals focus on pursuing positive future outcomes, whereas no relationship with the immediate subscale was found.

A healthy lifestyle encompasses many different types of behavior, and time orientation does not appear to be uniform across behaviors. Recently, van Beek et al. (2013) found eating behavior to be predicted best by a food-specific version of CFC, and exercising behavior to be predicted by CFC for exercising. A study by Hall, Fong, and Cheng (2012) is consistent with these results, showing that only a behavior-specific time perspective measurement was predictive of self-management behavior initiation among individuals newly diagnosed with Type 2 diabetes.Therefore it is hypothesized 
that behavior-specific time orientation measures are more predictive of actual behavior, and therefore more useful than commonly used general measures of time perspective.

The concept of CFC thus tries to capture the relative weight one gives to immediate, short-term implications of behavior versus the future, long-term implications. As such, this concept seems similar to the concept of delay discounting, which entails giving future outcomes less weight relative to more immediate outcomes (Frederick, 2006). CFC and discount rate are both related to how an individual perceives tradeoffs between the present and the future. Delay discounting reveals how the value of a reinforcer decreases as the delay to the receipt of the reinforcer increases, and a higher discount rate is indicative of a higher impulsivity (Ainslie, 1975). Research has shown that smaller immediate rewards are preferred over greater delayed rewards. Inability to delay gratification in a delay discounting task seems to reflect impulsive eating: the behavioral difficulties in overcoming short-term rewards of eating unhealthy food, despite the greater long-term benefits to health that could be obtained if immediate gratification is resisted. Research has shown that higher discount rate (e.g. quicker devaluation of the reinforcer when received later in time) combined with high food reward sensitivity was indeed predictive of a higher caloric consumption in a laboratory taste test (Appelhans et al., 2011; Rollins, Dearing, \& Epstein, 2010). Earlier studies also show some evidence for a relationship between Body Mass Index (BMI) and delay discounting (Jarmolowicz et al., 2014; Weller et al., 2008), although other studies failed to find this association (Nederkoorn et al., 2006; Rasmussen et al., 2010).

Most of the discounting research has been conducted on choices regarding money. However, for other reinforcers, research suggests stimulus-specific discounting patterns. Smokers, for example, discount cigarettes more than non-smokers (Field, Santarcangelo, Sumnall, Goudie, \& Cole, 2006), and alcoholics discount alcohol more steeply then non-alcoholics (Petry, 2001). In some cases there is little or no correlation between the discounting of different types of reward, indicating that these reward types are associated with different types of impulsivity (Green \& Myerson, 2013; Jimura et al., 2011). By showing different discount rates for different outcomes, it seems that how an individual discounts one outcome does not necessarily indicate how that individual will discount all types of outcome (Weatherly \& Derenne, 2011; Weatherly \& Ferraro, 2011; Weatherly \& Terrell, 2010). Given that discount rates can differ within and among individuals across various types of rewards (e.g. cigarettes, drugs, and alcohol), we wonder whether unhealthy eaters respond uniquely to food, such that they discount food more steeply than healthy eaters. Similar to CFC, discount rate is probably measured best with behavior-specific reinforcers in order to predict unhealthy eating. Indeed, there is some research to support this notion; Rasmussen et al. (2010) found discounting pattern for food to be related to body fat percentage, whereas a similar 
pattern in discount rate for money was not found. However, their design did not allow the direct comparison of food and money as the units and time intervals used for the delays were qualitatively different.

Because the constructs of time orientation and delay discounting are conceptually similar, the literature often uses these terms interchangeably. The overlap between these constructs is apparent: steep delay discounting seems analogous to a presentmoment orientation, choosing immediate pleasure over long-term reward. However, despite shared association and conceptual similarities, studies which investigated both constructs show that correlations between CFC and delay discounting measures are small (Adams \& Nettle, 2009; Daugherty \& Brase, 2010; Joireman et al., 2008), indicating these constructs, although related, are not the same. Therefore, it is important to further investigate the relationship between time perspective and discount rate, and their relative influence on specific behavior, namely eating. The goal of the current study, therefore, was threefold. First, the relationship of CFC and the food-specific version of the CFC with eating behavior was assessed. Since consideration of future consequences is thought to be measured best at a behavior-specific level (Hall et al., 2012; van Beek et al., 2013), it was hypothesized that healthy eating would be better predicted by CFCfood rather than CFC in general. Second, it was examined whether eating behavior is more strongly related to food-specific discount rate compared to discount rate for money (general discount rate). It was hypothesized that less healthy eating would be particularly related to elevated discounting patterns when choices were about highly palatable snack food. Third, the combined effects of discount rate and CFC on healthy eating were investigated. Individuals who do not tend to foresee future consequences, probably discount steeper and make the choice for immediate rewards more often. On the contrary, individuals who generally consider the long term consequences of their behavior will probably also discount less, reflecting a lower impulsivity. The association between CFC and discount rate was hypothesized to be strongest for the behaviorspecific measurements. These possibilities were therefore examined in the present study.

\section{METHODS}

\section{Participants}

A sample of 152 Dutch speaking participants (118 females) between the ages of 18-60 was recruited for this study. One participant was excluded from analyses because of missing data in the questionnaires, resulting in a final sample of 151 participants. The mean age of participants was 31.5 years $(S D=11.52)$. The majority of the sample $(77 \%)$ had completed, or was currently completing, a bachelor's degree or higher. Mean BMI 
was $25.9(S D=5.9)$. The link to the online questionnaire was spread via social media, forums about lifestyle, dieting and cooking, and via the student recruitment system for the psychology students of the University of Maastricht. The study was approved by the Ethical Committee of the Faculty of Psychology and Neuroscience at Maastricht University.

\section{Measures}

\section{Consideration of Future Consequences Scale and Consideration of Future Consequences Scale - Food}

In order to measure time orientation, participants filled in the 14-item Consideration of Future Consequences scale (CFC; Joireman et al., 2012). The CFC consists of the Immediate- and Future-subscale, both consisting of 7 items. Joireman et al. (2012) recently added 2 items to the original 5-item Future-subscale to improve the internal consistency. Participants indicate their agreement with the statements on a 7-point Likert scale, ranging from $1=$ "totally disagree" to $7=$ "totally agree." To determine a total score, items of the immediate-subscale were reverse scored and the mean of all items was taken, with higher overall scores indicating greater concern with the future. In addition to assessing the influence of total score on eating behavior, it was further explored whether associations were mainly driven by the Immediate-subscale, the Future-subscale, or both. ${ }^{1}$ An example for an item of the Immediate-subscale is "I only act to satisfy immediate concerns, figuring the future will take care of itself." An example question for the future-subscale is "I consider how things might be in the future, and try to influence those things with my day to day behavior."

In addition to the CFC, participants also completed the CFC-food, an adapted version of the CFC specific about eating behavior, developed by van Beek et al. (2013). An example of a modified item of the Immediate-subscale is "I only choose my food to satisfy immediate needs, figuring the future will take care of itself." An example question for the Future-subscale is "I consider how my health might be in the future, and try to influence my health with my day to day eating behavior." For the current study the two new items of Joireman et al. (2012) of the Future-subscale were also adapted and added.

\section{Monetary choice Questionnaire and modified version for snack food}

The Monetary Choice Questionnaire (MCQ; Kirby, Petry, \& Bickel, 1999) was used to measure discount rate. This questionnaire consists of 27 questions, asking the respondent to choose either a smaller, immediate reward or a larger, delayed reward. Dollar signs were replaced by euro-signs to make the rewards more appropriate for Dutch participants. The discount rate of each participant was calculated by estimating the k-value (Kirby, 2000). K is a free parameter which reflects the degree of discounting 
(i.e., higher $\mathrm{k}$ values represent higher sensitivity to delay, or greater impulsivity). Using the formula $V=A /(1+k D)$, in which $V$ is the indifference point, $A$ is the delayed reward, and $D$ is the delay in days, $k$ is calculated for each participant for small, medium, and large rewards (Kirby, 2000). An overall discounting parameter was then calculated then for each participant by taking the geometric mean of the k-value assigned for small, medium and large rewards (Kirby et al., 1999). An example of an item measuring discount rate is: "Would you prefer $€ 55$ today or $€ 75$ in 61 days?" As a measure of internal reliability, a consistency value was calculated for each subject as the proportion of responses that were consistent with subject's k-value. Hypothetical rewards were used in the current research, but participants were asked to act like they would receive real rewards. Earlier studies show no significant differences in discounting between real and hypothetical conditions (Johnson \& Bickel, 2002; Lagorio \& Madden, 2005; Madden, Begotka, Raiff, \& Kastern, 2003).

In order to obtain discount rate for snack food, the MCQ was modified by replacing the money with snack foods. Participants first indicated their favorite snack food item (crisps, cocktail nuts, M\&M's or jelly sweets), and this food item was then used in the delay discounting task. The snacks were selected for their small size, which were evaluated as manageable portions that could be eaten in a single serving. Amounts and delays were kept consistent with the original questionnaire, but one Euro was replaced by one piece of snack/candy of the chosen favorite snack. For example, an item for an individual who chose M\&M's as his favorite snack would be: "Would you prefer 55 M\&M's today, or 75 M\&M's in 61 days?"

\section{Hunger and taste rating of snack food}

Participants were asked to indicate their current hunger on a 7-points Likert scale, ranging from $1=$ "Not at all hungry" to $7=$ "Extremely hungry". They rated the taste of the snack food (crisps, cocktail nuts, M\&M's and jelly sweets) on a 7-point Likert scale, ranging from $1=$ "Not at all tasty" to $7=$ "Very tasty".

\section{Body Mass Index (BMI)}

Self-reported height and weight of each participant was assessed to calculate BMI (kg/ $\left(m^{2}\right)$.

\section{Eating behavior}

In order to determine healthy eating, participants completed a questionnaire about their eating behavior during the past two weeks (Baker, Little, \& Brownell, 2003; Kuijer \& Boyce, 2012, 2014). The questionnaire consisted of the following items: how often someone (1) ate appropriate portions of food (not too much or too little), (2) ate in a 
balanced way, with a lot of fruits and vegetables, (3) ate junk food (potato chips, desserts, sweets, candy bars, etc.), (4) overate (kept eating while already satiated), and (5) ate breakfast. Items were scored on a 5-point scale, ranging from "every day" to "less than once a week". Items concerning unhealthy eating (item 3 and 4) were reverse scored, resulting in one total score for eating behavior, with higher scores indicating healthier eating. With regard to validity, Kuijer and Boyce (2012) found the recall of the five eating behaviors to be highly correlated with a two week diary report of those behaviors (See Kuijer \& Boyce (2012) for details). According to Kuijer and Boyce (2014), the scale has satisfactory internal consistency, with a reported Cronbach's alpha coefficient of .67. In the current study, Cronbach's alpha was .70.

\section{Procedure}

Participants were recruited for an online study about eating behavior and the future. Interested participants could follow a provided link directly to the study questionnaires. They first indicated how hungry they were at the moment, selected their favorite snack food from the list provided, and indicated how much they liked the snack food. Then participants completed the MCQ (Kirby et al., 1999), the modified MCQ-food, the CFC (Joireman et al., 2012) and CFC-food (van Beek et al., 2013). The order of delay discounting (MCQ and MCQ-snack) and CFC (general and CFC-food) measures was counterbalanced between participants. Participants then completed the questionnaire about their eating behavior in the last two weeks, and reported demographic data including height and weight. All questionnaires were provided in Dutch. Completion took about 20 minutes. In return for their participation, participants could enter a lottery to win an IPod Nano.

\section{Data analyses}

First, zero-order correlations between predictors, BMI and healthy eating were assessed. Partial correlations controlled for demographical data and the influence of current hunger and taste rating of chosen snack food were also explored. Next, predictors were entered in a multiple regression analysis to compare the unique influence of each predictor on healthy eating.

\section{RESULTS}

\section{Preliminary results}

Preliminary analyses indicated that based on Kirby's (2009) exclusion criteria, five participants showed inconsistent discounting, evidence by a consistency rate under 0.75 for assigned k-value for one or more of the categories of reward, and were 
therefore excluded from further analyses. No significant differences in demographic data, BMI, or eating behavior were observed between the excluded subjects and the remaining sample. In Table 1, demographic data for the final sample of 146 participants are provided. Scale properties (CFC, CFC-food, MCQ, MCQ-snack and healthy eating) are provided in Table 2. Results showed a high consistency rate for assigned k-value. A Wilcoxon Signed Rank Test revealed a significant difference between discount rate for snacks and discount rate for money, $z=-7.50, p<.001$, indicating overall stronger discounting for snacks then money. $32.2 \%$ of all participants received a k-value of .25 for snack food, which indicated maximal discounting. Because data were not normally distributed for k-values, their natural logs were used for further analyses. Mean current hunger was $3.12(S D=1.73)$. Mean taste rating of chosen snack food was 6.01 (SD = 1.09), which shows that participants in general liked the chosen snack.

TABLE 1 Demographic characteristics of the final sample

\begin{tabular}{lll} 
& M & SD/\% \\
\hline Age & 31.14 & 11.50 \\
Gender & Male & $23.3 \%$ \\
& Female & $76.7 \%$ \\
Education & High School & $21.2 \%$ \\
(some or completed) & College/University & $70.6 \%$ \\
& Graduate studies & $8.2 \%$ \\
BMI & $<18.5$ & $3.4 \%$ \\
& $18.5-24.9$ & $51.4 \%$ \\
& $25-29.9$ & $26.0 \%$ \\
& $30-34.9$ & $11.0 \%$ \\
& $>35$ & $8.2 \%$
\end{tabular}

Note: $N=146 ; \mathrm{BMI}=$ Body mass index

TABLE 2 Scale properties of study variables

\begin{tabular}{|c|c|c|c|c|}
\hline Measure & $M$ & SD & a/internal consistency & $N$ of items \\
\hline CFC & 4.60 & 0.73 & .82 & 14 \\
\hline CFC-Immediate & 25.71 & 6.27 & .74 & 7 \\
\hline CFC-Future & 34.05 & 5.76 & .74 & 7 \\
\hline CFC-food & 4.61 & 0.88 & .86 & 14 \\
\hline CFC-Immediate food & 23.69 & 7.50 & .84 & 7 \\
\hline CFC-Future food & 32.21 & 6.74 & .79 & 7 \\
\hline MCQ & $0.0141^{\mathrm{a}}$ & 0.03 & $96.85 \%^{\mathrm{b}}$ & 27 \\
\hline MCQ-snack & $0.1075^{\mathrm{a}}$ & 0.11 & $96.35 \%^{\mathrm{b}}$ & 27 \\
\hline Healthy eating & 19.38 & 3.38 & .70 & 5 \\
\hline \multicolumn{5}{|c|}{$\begin{array}{l}\text { Note. } \mathrm{CFC}=\text { Consideration of Future Consequences, } \mathrm{MCQ}=\text { Monetary Choice Questionnaire, } \mathrm{BMI}=\text { Body Mass } \\
\text { Index. }\end{array}$} \\
\hline
\end{tabular}




\section{Zero-order correlations}

The outcome variable, self-reported healthy eating, did not correlate significantly with age $(r=.08, p=.35)$, gender $(r=-.02, p=.85)$ or education $(r=.11, p=.21)$. Discount rate for snacks was not related to current hunger $(r=-.01, p=.93)$ or taste rating of the chosen snack $(r=.15, p=.08$ ). Table 3 presents zero-order correlations between predictors, BMI and healthy eating. First, Table 3 shows that the general measures are moderately correlated with their behavior-specific variants: CFC and CFC-food are moderately correlated, while MCQ and MCQ-snack are also moderately correlated. CFC-food shows a small negative correlation with MCQ and with MCQ-snack. Further inspection of results reveals that the relation with $M C Q$ is driven by the future-subscale only of the CFC-food, while the correlation of CFC-food with MCQ-snack is driven by both subscales. Our general measures, CFC and MCQ did not correlate with healthy eating. The adapted version of CFC, CFC-food, shows a strong correlation with eating behavior, with a significant relationship between both the Immediate and Future-subscale to healthy eating. MCQ-snack shows a marginally significant negative correlation with healthy eating $(r=-.16, p=.06)$. BMI shows a positive correlation with MCQ and a marginally significant negative correlation with healthy eating $(r=-.16, p=.06)$.

TABLE 3 Zero-order intercorrelations ${ }^{\mathrm{a}}$ among predictors, body mass index and healthy eating

\begin{tabular}{|c|c|c|c|c|c|c|c|c|c|c|}
\hline Measure & 1 & 2 & 3 & 4 & 5 & 6 & 7 & 8 & 9 & 10 \\
\hline 1. CFC & - & & & & & & & & & \\
\hline 2. CFC-immediate & $-.87^{* * *}$ & - & & & & & & & & \\
\hline 3. CFC-future & $.84^{* * *}$ & $-.46^{* * *}$ & - & & & & & & & \\
\hline 4. CFC-food & $.40^{* * *}$ & $-.37^{* * *}$ & $.32 * * *$ & - & & & & & & \\
\hline 5. CFC-immediate food & $-.42^{* * *}$ & $.48^{* * *}$ & $-.24^{* *}$ & $-.88^{* * *}$ & - & & & & & \\
\hline 6. CFC-future food & $.26^{* *}$ & -.14 & $.32^{* * *}$ & $.85^{* * *}$ & $-.49 * * *$ & - & & & & \\
\hline 7. $\mathrm{MCQ}$ & -.05 & .05 & -.04 & $-.18^{*}$ & .11 & $-.21^{*}$ & - & & & \\
\hline 8. MCQ-snack ${ }^{b}$ & -.12 & .07 & -.16 & $-.16^{*}$ & .15 & -.14 & $.33^{* * *}$ & - & & \\
\hline 9. BMI & -.01 & -.02 & -.03 & -.12 & .12 & -.09 & $.24^{* *}$ & .08 & - & \\
\hline 10. Healthy eating & .07 & .00 & .12 & $.53^{* * *}$ & $-.48^{* * * *}$ & $.43^{* * *}$ & -.12 & -.16 & -.16 & - \\
\hline \multicolumn{11}{|c|}{$\begin{array}{l}\text { Note: } \mathrm{CFC}=\text { Consideration of Future Consequences, } \mathrm{MCQ}=\text { Monetary Choice Questionnaire, } \mathrm{BMI}=\text { Body Mass } \\
\text { Index. Two-tailed Pearson's correlations } \\
\text { a Partial correlations corrected for current hunger and taste rating of chosen snack food did not change the } \\
\text { pattern of results and are therefore not reported. } \\
{ }^{\mathrm{b}} \text { For MCQ-snack, because of skewness of data, non-parametric Spearman correlations are reported. } \\
{ }^{*} p<.05 \text {. } \\
{ }^{* *} p<.01 \text {. } \\
{ }^{* * *} p<.001\end{array}$} \\
\hline
\end{tabular}




\section{Partial correlations}

When exploring partial correlations controlling for the influence of age, gender and education level, the correlation between BMI and CFC-food approached significance ( $r$ $=-.15, p=.07)$. Healthy eating showed a small but significant negative correlation with BMI ( $r=-.18, p=.04)$. For CFC, MCQ and MCQ-snack the pattern of outcome did not change and results are therefore not reported.

\section{Predictive models}

Next, in order to compare the unique influence of each predictor on eating behavior, a multiple regression analysis was conducted ${ }^{2}$. Because no significant correlations were found between CFC and outcome variables or discount measures, the regression analysis was conducted including only the Immediate and Future-subscale of CFCfood, MCQ, and MCQ-snack. When all the predictors were entered, the total variance explained by the model as a whole was $28.6 \%, F(4,141)=14.13, p<.001$. Both the CFC-food Immediate $(\beta=-.35, p<.001)$ and the CFC-food Future subscale $(\beta=.24, p$ $=.01$ ) significantly predicted healthy eating, such that higher scores on the CFC-food Immediate subscale predicted less healthy eating whereas higher scores on the Future subscale predicted healthier eating. There was no significant contribution of MCQ ( $\beta=$ $-.00, p=.98)$ or MCQ-snack ( $\beta=-.09, p=.24)$. These results show that the prediction of healthy eating is not weakened by adding MCQ and MCQ-snack. When all predictors are considered together in the model, CFC-Immediate and CFC-Future food stay the single most predictive factors of healthy eating.

\section{DISCUSSION}

In the current study the relationships between healthy eating, CFC and discount rate - in general and with behavior specific measurements - were investigated. The results show that a food-specific measure of consideration of future consequences is related to healthy eating, such that individuals concerned with immediate consequences report less healthy eating, whereas those who are more concerned with future health report healthier eating. Discount rate did not correlate with healthy eating in the current sample, although steeper discounting for money was related to BMI. Discounting of snack food appeared to be related to unhealthy eating; however this relationship did not reach statistical significance. After controlling for demographic data and discount rate, CFC-food remains the main predictor of healthy eating. The weak correlations between CFC-food and discount rate in the current study suggest these are two distinct, but not entirely independent, constructs in the prediction of healthy eating. 
Although researchers recently found a significant relationship between healthy eating and the CFC-future subscale (Joireman et al., 2012), this relationship was not established in the current study. However, the small correlation reported by Joireman et al. (2012) was comparable to the current results. Importantly, the current results support our hypothesis that for the purposes of behavior prediction, CFC should be tailored to the behavior at interest and not be measured as a general construct, in line with the outcomes of van Beek et al. (2013). Further, both the Immediate and Future subscales of the CFC-food contributed equally to healthy eating. Joireman et al. (2008) proposed two competing models, based on the distinction between CFC-immediate and CFC-future. The 'susceptibility model' posits that a high level of CFC-immediate makes one prone to self-control failure, whereas the 'buffering model'states that a high level of CFC-future can prevent self-control failure. Though this is not a primary concern in our study, the current results contribute to this debate by confirming the better fit of the two-factor model instead of the one-factor model of CFC. However, in terms of behavioral outcomes, both subscales seem to be related to healthy eating to an equal extent. While in the study of van Beek et al. (2013) only the CFC-Immediate subscale was related to healthy eating, the current study suggests that both mechanisms could jointly contribute to eating behavior.

In the current sample no relation between discount rate and healthy eating was observed, which is inconsistent with previous studies (Appelhans et al., 2011; Rollins et al., 2010). However, these previous studies included an ad libitum taste test to measure caloric intake as outcome variable, while we measured retrospective self-report of healthy eating. Most importantly, previous results showed that food reinforcement was a more powerful predictor of energy intake than discount rate, and discount rate was only a predictor of caloric intake for individuals who were high in food reinforcement. The current study did not take food reinforcement into account, which can explain why no relationship with healthy eating was found. BMI, however, did show a small but significant correlation with discount rate for money, which is in line with earlier research (Jarmolowicz et al., 2014; Weller et al., 2008).

However, our results for discount rate of snack food warrant cautious interpretation, as the restricted variability in observed discount rate may have contributed to a ceiling effect. Specifically, it seems that a majority of participants always choose to receive an immediate food reward rather than waiting for a larger reward, regardless of waiting time or amount of food offered. No further distinction in discount rate can be made between those participants, because all showed maximum discounting on the current task. Future research should use a food-specific discounting task with no restriction of range to allow for a deeper investigation of the hypothesized relationship between discount rate and healthy eating. Importantly, it seems that with regard to 
snack foods, the relationship between discounting future food and self-control is not as straightforward as when choices are about money. The real-world choice for an obese person trying to lose weight is not to eat a small amount now or a larger amount later. By choosing the direct gratification in our current task, participants are choosing an outcome that will ultimately be healthier (i.e., consuming fewer snacks) compared to choosing the larger, delayed option. This may be one reason MCQ-snack was not closely related to the outcome of healthy eating. Although earlier research on this topic measured discount rate with different amounts of food (Odum, Baumann, \& Rimington, 2006; Odum \& Rainaud, 2003) and has shown discount rate for bites of food to be related to percentage body fat (Rasmussen et al., 2010), this does not generalize to choices in daily life. Individuals should avoid unhealthy foods now in order to lose weight and reduce the risk of disease in the future. As suggested by Epstein et al. (2010), discount measures with different reinforcers (e.g. food now or health or losing weight later) could therefore be more predictive of behavior. In a more relevant delay discounting task the snack would be available immediately, and the weight loss would be available at different times in the future.

Measurements of discount rate and CFC were at best only weakly correlated. Some variance in the small correlation between delay discounting and CFC can be explained by the difference in measurement: CFC was measured by self-report, whereas delay discounting was a performance-based questionnaire. Food-specific CFC-Immediate and CFC-Future were the main predictors of eating behavior in the current study, and are therefore interesting targets for intervention. The challenge for future research is to promote a future orientation with regard to health among individuals high on CFCImmediate and low in CFC-Future. The current results are cross-sectional, but Hall and Fong (2003) showed that a brief time perspective intervention aimed at enhancing longterm thinking about physical activity led to increased activity, suggesting that futureoriented thinking may indeed be causally related to the uptake of health behavior. Research shows that discount rate can be altered by episodic future thinking; that is, individuals can learn to make less impulsive decisions by incorporating visualization of the future in the decision process (Benoit et al., 2011; Daniel, Stanton, \& Epstein, 2013a, 2013b; Lin \& Epstein, 2014; Peters \& Büchel, 2010). Benoit et al. (2011) found evidence for the moderating influence of CFC, with those low in CFC showing the greatest decrease in discount rate after episodic future thinking. In addition to altering discount rate, episodic future thinking also altered food intake in a recent study, resulting in less caloric consumption (Daniel et al., 2013b). This is consistent with the idea that stimulating thinking of the future promotes healthy eating. This represents an interesting avenue for future research; that is, given the current results indicating that unhealthy eaters do not consider their future health when making food-related 
decisions, it could be that an intervention based on future thinking may have an even stronger influence on food intake when it is specifically aimed at eating behavior and future health. It is unclear, however, what specific future consequences participants have in mind when completing the CFC-food; for example, participants could imagine either positive or negative health (van Beek et al., 2013). Earlier research has shown that craving for cigarettes and food could be reduced by an intervention specifically focused either on the short-term consequences associated with consuming the item (e.g. "it will taste good") or on the long-term health consequences associated with regular consumption (e.g. "I may get lung cancer" (Kober, Kross, Mischel, Hart, \& Ochsner, 2010). Although their primary goal was to reduce craving for smokers, all participants reported significantly reduced food cravings when focusing on the long-term consequences associated with repeated consumption of unhealthy foods (Kober et al., 2010). Kober et al. (2010) show that considering negative health consequences can be an effective strategy, though focusing on future positive health outcomes (e.g. the delayed reward) could also prove beneficial.

The current study was not without limitations. All results are based on self-report. BMI was calculated based on self-reported weight and height. Research shows that people tend to underestimate their weight a bit and slightly overestimate their height, although self-reported and measured BMI are typically highly correlated (Spencer, Appleby, Davey, \& Key, 2002). BMI was not correlated with CFC in general or with our food-specific measurement in the current study. Nonetheless, unhealthy eating does put a person at risk of becoming overweight in the future, while healthy eating could be protective for becoming overweight. Importantly, the finding that BMI and eating behavior were not strongly correlated deserves attention and questions the current measure of eating behavior. A post hoc explanation could be that we did not take into account whether participants were on a current diet. This could have influenced their eating behavior and partly account for this lack of relationship. Future studies measuring actual eating behavior could strengthen and extend current findings.

\section{CONCLUSION}

In conclusion, the current study demonstrated a strong relationship between a foodspecific version of CFC-Immediate and CFC-Future and healthy eating. Behaviourspecific individual differences in time orientation thus contribute to healthy eating patterns: Considering the future consequences of food consumption is strongly related to healthier eating, while consideration of more immediate outcomes of food intake is related to less healthy eating patterns. This pattern of results was only found using a food-specific version of time orientation, indicating that time orientation measures 
should be tailored to the outcome behavior. Given the observational character of the current study, future studies with experimental designs and measurements of actual behavior will be able to make stronger claims about the relation between these investigated mechanisms. The challenge now is to develop effective interventions to help people delay gratification and make healthier food choices. Greater understanding of the mechanisms behind unhealthy eating, including time orientation, could aid in the prevention of obesity by targeting subjects who are at risk, and changing their food habits in a way that prevents eventually becoming obese.

\section{ACKNOWLEDGEMENTS}

This project was funded by Eatwell (http://www.um-eatwell.nl/). The sponsors had no further role in study design; in the collection, analysis and interpretation of data; in the writing of the report; or in the decision to submit the paper for publication.

\section{Footnotes}

1 Confirmatory Factor Analyses was performed in order to compare the fit of a two- or one-factor model underlying CFC and CFC-food. For the CFC, the two-factor model showed a reasonable fit to the data, $\mathrm{X} 2(76)=143.78, p<.001$, Goodness of Fit Index $(\mathrm{GFI})=.875$, Comparative Fit Index $(\mathrm{CFI})=.878$, root mean square error approximation $(\mathrm{RMSEA})=.078$ (lower limit $[\mathrm{LL}]=.059$, upper limit $[\mathrm{UL}]=.098)$. The one-factor model fit to the data poorly, $X 2(77)=202.54, p<.001$, $\mathrm{GFI}=.808, \mathrm{CFI}=.773, \mathrm{RMSEA}=.106(\mathrm{LL}=.088, \mathrm{UL}=.124)$. For CFC-food, the two-factor model also showed a reasonable fit to the data, $\mathrm{X} 2(76)=126.38, \mathrm{p}<.001$, Goodness of Fit Index (GFI) $=.885$, Comparative Fit Index $(\mathrm{CFI})=.929$, root mean square error approximation (RMSEA) $=$ .068 (lower limit $[\mathrm{LL}]=.046$, upper limit $[\mathrm{UL}]=.088$ ), whereas the one-factor model fit the data poorly, $\mathrm{X2}(77)=232.25, p<.001, \mathrm{GFI}=.769, \mathrm{CFI}=.783, \mathrm{RMSEA}=.118(\mathrm{LL}=.101, \mathrm{UL}=.135)$. The two-factor model fit the data for the CFC significantly better than the one-factor model, X2 $\triangle$ (1) $=58.77, \mathrm{p}<.001$. For CFC-food, the two-factor model also showed a significant better fit then the one-factor model, $\mathrm{X} 2 \Delta(1)=105.87, p<.001$.

2 Conducting a hierarchical regression analyses with demographical data (age, gender and education level), current hunger and taste rating of chosen snack food entered in the first step did not change the pattern of outcomes and results are therefore not reported. 



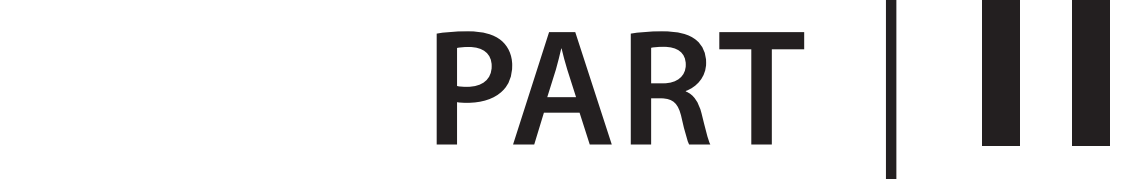

Manipulating Cognitive Control

Episodic Future Thinking, Shifting and Working Memory Training 



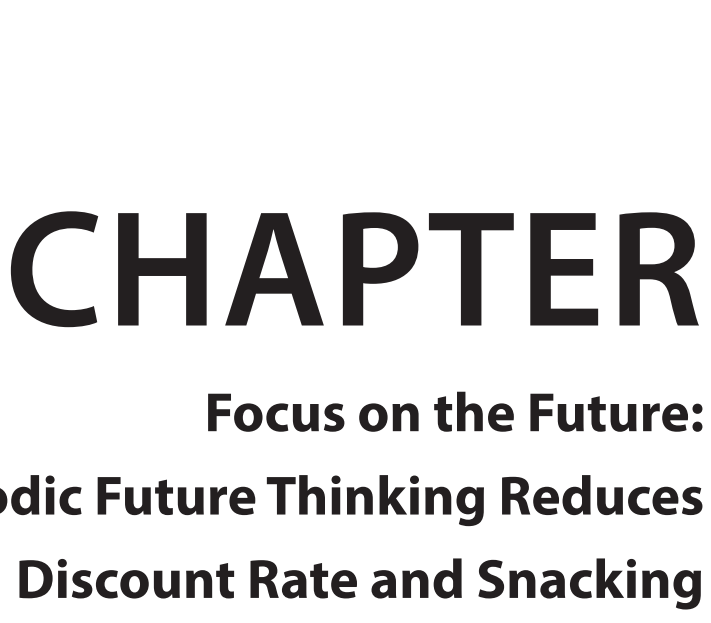

Published as: Dassen, F. C. M., Jansen, A., Nederkoorn, C., Houben, K. (2016).

Focus on the future: Episodic future thinking reduces discount rate and snacking. Appetite, 96, 327-332. https://doi.org/10.1016/j.appet.2015.09.032 


\section{ABSTRACT}

Obesity seems related to a preference for immediate gratification. By changing this focus on short term benefits to a more future-oriented outlook, delay discounting (impulsive decision making) can be changed by a manipulation of episodic future thinking (EFT). EFT comprises a vivid mental simulation of general future experiences. EFT may also affect consumption of unhealthy foods, which can be seen as a choice for immediate gratification. Recent research shows that future orientation should be tailored to the behavior at outcome. We therefore hypothesize that the effectiveness of EFT on food intake could be enhanced by making the content food-related. We conducted a 2 (future vs past thinking) by 2 (food vs non-food related thoughts) between-subject design experiment in female undergraduates ( $N=94)$, to compare the efficacy of EFT versus the recalling of episodic past events in reducing discount rate and caloric intake. Content of imagery was either unrestricted or food-related. Participants engaged in EFT or control episodic imagery while snacks were offered to freely consume, and next the Monetary Choice Questionnaire was completed as a measure of delay discounting, while again being engaged in EFT or control imagery. Both types of EFT reduced delay discounting, however, only food-related EFT lead to more restricted caloric consumption. Thus, we found evidence that EFT reduced discount rate during decision making. However, in order to restrict caloric intake, EFT should entail food-related imagery. As discount rate and caloric intake were not related in the current sample, the underlying mechanism remains to be discovered. Results however suggest that EFT is a promising technique to resist immediate gratification.

Key words: Eating Behavior; Episodic Future Thinking; Time Orientation; Delay Discounting; Reducing Impulsivity 


\section{INTRODUCTION}

In the last decades, people became increasingly heavier and physically less fit, and the prevalence of obesity has increased worldwide (Finucane et al., 2011; Flegal et al., 2012). When a person's energy intake is greater than one's energy consumption, weight gain will occur (Hill, Melanson, \& Wyatt, 2000). Why is it so difficult for some to resist the temptation of palatable food? The taste of high sugar and high fat foods is highly enjoyable (Drewnowski \& Greenwood, 1983) and eating high caloric foods is very rewarding at the time of consumption: the immediate contingencies are positive (Volkow, Wang, \& Baler, 2011). When choosing what to eat, we have to weigh immediate pleasures vs. long-term health implications (e.g. healthy body weight and reduction of health risks) (Hall \& Fong, 2007). When one is mainly focused on immediate pleasures, this could lead to palatable, though unhealthy, food choices. Studies on preferences for immediate reward frequently used a delay discounting task, in which individuals have to choose between a more valuable reward after a period of delay and a less valuable reward which is directly available (e.g. Bickel \& Marsch, 2001). Dual-system models of decision-making (e.g. Bechara, 2005; Bickel et al., 2007) posit that delay discounting reflects the relative balance in activation between two competing neurobiological systems. The appetitive, impulsive system values immediate reinforcers, whereas the inhibitory, executive system is needed for the inhibition of the impulsive system to maximize long-term gains (Bickel et al., 2007). Someone who chooses relatively more often for the immediate smaller reward is thus considered to be more impulsive, whereas someone who is more willing to wait for a bigger reward, places more emphasis on benefits in the future (Bickel et al., 2007). Research has shown that obese individuals are more affected by this period of delay than subjects with a healthy weight, and a higher discount rate (i.e., less willingness to wait for future benefits) is related to a higher Body Mass Index (BMI) (Epstein et al., 2014; Jarmolowicz et al., 2014; Weller et al., 2008). A higher discount rate is also predictive of higher calorie intake in obese women (Appelhans et al., 2012) and children who are having more difficulty delaying reward, lost less weight during a weight loss intervention (Best et al., 2012). Changing this concern with immediate reward into a more future-oriented outlook could therefore be useful in order to promote the choice of healthy foods and thereby facilitate a healthy weight.

Episodic future thinking (EFT) is one strategy to shift one's preference from immediate gratification to delayed rewards (Peters \& Büchel, 2010). EFT refers to the possibility of humans to project themselves forward in time and to pre-experience future events (Atance \& O'Neill, 2001). Simulation of a future event is a relatively unconstrained task that places many demands on executive functions (Schacter, Addis, \& Buckner, 2008) and integrating episodic future images in choice behavior increases activation in the 
executive system (Peters \& Büchel, 2010). This increased activation of the executive system could be resulting in an increased choice of delayed rewards (Koffarnus, Jarmolowicz, Mueller, \& Bickel, 2013). It is suggested that this capacity for EFT gives people the ability to counteract their natural disposition to short-term decision making and to make restrained choices with long-term benefits. EFT thus has adaptive utility resulting in higher evolutionary fitness (Boyer, 2008). Thoughts about the future are frequently used in daily life to plan actions and make decisions (D'Argembeau, Renaud, \& Van der Linden, 2011). EFT possibly heightens connectedness and reduces perceived distance between the future and now (Koffarnus et al., 2013) and it seems to help in assessing the value of delayed rewards (Bar, 2010). Interestingly, Benoit et al. (2011) showed the greatest decrease in discount rate after EFT for those scoring low on the Consideration of Future Consequences Scale (Strathman et al., 1994), a measure of time orientation which entails the extent to which people consider immediate versus future consequences of potential behaviors. Thus, these results indicate that a stimulation of EFT is the most beneficial to those who in general do not take future outcomes in account.

Engaging in EFT during decision making may help individuals to delay gratification in food choice (Daniel et al., 2013a). Weight management is in general considered more important than eating snacks, until snacks are available and negative consequences are discounted (Boyer, 2008). In earlier research, EFT reduced caloric intake in obese women (Daniel et al., 2013b) and overweight children (Daniel, Said, Stanton, \& Epstein, 2015). These results suggest that EFT could indeed be a useful technique to endorse long term consequences and thereby control impulsive eating. It is important to note that Daniel and colleagues (Daniel et al., 2015; Daniel et al., 2013b) found effects of EFT on eating behavior even though the EFT manipulation was unrelated to eating. Specifically, participants were encouraged to imagine vivid positive future events, whereby the content of imagery was not restricted. It thus seems that the imagined future events during EFT do not necessarily have to be related to the discounting task or to eating episodes: Considering general future events was enough to lead individuals into better eating-related choices in the present.

However, there is reason to believe that future orientation should be tailored to the behavior of interest. Someone can be more future-oriented in one aspect of his behavior, but not in another (van Beek et al., 2013). Earlier research has shown that only a food-specific measure of time orientation was related to healthy eating patterns (Dassen, Houben, \& Jansen, 2015; Hall et al., 2012; van Beek et al., 2013): unhealthy eaters were focused on immediate benefits only with respect to food and health, whereas healthy eaters took future health-related consequences of their eating behavior into account (Dassen et al., 2015). These results suggest that the effectiveness of EFT could be enhanced by making the content food-related. 
The goal of the current study is therefore to examine whether food-related EFT is more effective in promoting healthy dietary choices compared to general EFT. Participants were instructed to think vividly about the future or about past events and whether the thoughts were general or food-related in a $2 \times 2$ between subjects design. Following the episodic thought manipulation, we assessed discount rate and caloric intake. It was expected that the EFT conditions would show a reduction in both delay discounting and food intake relative to the control conditions. As a reduction in discount rate is suggested to be the underlying mechanism leading to a more restricted intake, we hypothesized that the effect of EFT on food intake is mediated by discount rate. In addition, it was hypothesized that food-related future imagination would be more effective in reducing caloric intake than general EFT.

\section{METHODS}

\section{Participants}

Ninety-five female undergraduates of Maastricht University between the ages of 1830 were recruited using advertisements for a study about the effects of deprivation on visualization. One participant was considered an outlier on the dependent variable (caloric intake > $3 S D$ ) and therefore excluded from all analyses. Therefore, our final sample consisted of 94 participants.

\section{Procedure}

Participants were asked to refrain from consuming anything except for water for two hours before participation. All appointments were scheduled between $11 \mathrm{am}$ and $6 \mathrm{pm}$. At arrival, participants signed the informed consent, completed demographical data, rated their current hunger, reported the time since their last meal and completed some filler-questionnaires. Next a 'break' was introduced, in which snacks and water were provided to freely consume while the manipulation, presented as a writing assignment (either EFT or control; see description of manipulation below) had to be completed. After fifteen minutes, the experimenter came back and removed the food. When the participant did not finish yet, a maximum of 5 extra minutes to work on the assignment was provided (15 participants; 16\%), while the experimenter waited in the room. Everyone was finished after this extra time. Next, a computerized questionnaire to assess discount rate was completed. Subsequently, participants rated on a 7-point Likert scale how much they thought about and how vividly they imagined the events while completing the questionnaire. Next, the Consideration of Future Consequences Scale food version and Restraint Scale were completed. At the end of the study, participants' weight and length were measured, and they were fully debriefed. Completion took 
about 50 minutes, and participants received one course credit or a voucher of $€ 7.50$ as a reward. The study was approved by the ethical committee of the Faculty of Psychology and Neuroscience of Maastricht University.

\section{Materials and measures Manipulations}

Participants wrote about either events planned in the future (Episodic Future Thinking: EFT) or about past events as control (Episodic Past Thinking: EPT). Participants either had to come up with unrestricted or food-related events, depending on the condition they were allocated to. Participants received the following instructions (instructions for the conditions who wrote about past events are shown between parentheses):

"We want to ask you to imagine events that realistically could happen or that you have already planned in the future (in a week, two weeks, a month, six months) [recall events that have taken place recently (one day ago, two days ago, a week ago, a month ago)]. This may for example be a getaway or something study-related; Think of something that applies to you. Experience this event already in mind [Re-experience this event in mind]. Consider as many details of this event as possible (what do [did] you do, where are [were] you, who are [were] with you, accompanying feelings). These images will be used in the next exercise. Describe the situation below."

For the food-related EFT and EPT condition, it was added to the instruction that the events had to be food-related. As an example it was stated that this event could for instance be a dinner with friends or cooking an elaborate meal. Participants were also asked to consider how healthy the described food-related event is/was. Next, the participants rated every image on a 7-point Likert scale ( 1 = not at all; 7 = very much) on realism, details, vividness, positivity, difficulty to imagine, and how future-oriented the event was.

\section{Caloric intake}

Participants were provided with a bowl of chocolate chip cookies (+/- 110 gram; 497 $\mathrm{kcal} / 100 \mathrm{gram})$, a bowl of M\&M's (+/- 200 gram; $485 \mathrm{kcal} / 100 \mathrm{gram})$, a bowl of syrup waffles (+/- 160 gram; $457 \mathrm{kcal} / 100$ gram) and a bottle of water. Participants were told that for study purposes it was no longer needed for them to be deprived of food, and since they had not eaten for at least two hours, some snacks and water were now provided to freely consume, while they were preparing at ease for the next assignment. They were told they could eat as much or as little as they wanted. Participants were not aware of the fact that the food was weighed before and afterwards and that caloric intake was calculated. 


\section{Monetary Choice Questionnaire}

In order to assess the ability to delay gratification, participants completed a computerized version of the Monetary Choice Questionnaire (MCQ; Kirby et al., 1999). This questionnaire consists of 27 questions, asking the respondent to choose either a smaller, immediate monetary reward or a larger, delayed monetary reward. An example of a question is "Would you prefer $€ 14$ today, or $€ 25$ in 19 days?" The timeframes of the future events approximated the time delays specified on the MCQ (7-186 days), based on time intervals specified by Lin and Epstein (2014). Participants received the following instructions regarding the EFT or control-EPT manipulation:

"This questionnaire consists of each a visualization task, where you have to imagine the events you just wrote about and subsequently a choice where you are asked to choose between an immediate reward and a higher reward you will only receive after a certain number of days. You will be asked which of the two rewards you prefer. The rewards include a certain amount of money. You will not really receive the amount of money you choose, but we want to ask you to make your decisions as if you actually would receive the money. Please choose the option that you would prefer to receive, and not what you think we want you to choose. They are a total of 27 choices. You do not have to base your choice upon the imagined event; you have just have to vividly imagine that this event is really happening, and next you can choose your preferred reward."

The geometric mean of the k-value (the degree of discounting) was calculated afterwards by use of an automated scoring spreadsheet made available online by Kaplan et al. (2014). See Kirby (2009) for more details about estimating the k-value. To normalize the distribution of scores, a natural-log transformation of $k$ was used for all statistical analyses.

\section{Restraint Scale}

In order to discover the extent to which participants try to restrain or control their food intake, the Restraint Scale (RS; Herman \& Polivy, 1980), which assesses weight concerns, weight fluctuations and self-reported attempts to diet, was used in this study. The RS is a self-report questionnaire consisting of 10-items, with possible scores ranging from 0 to 35. Higher scores are indicative of an increased intention to restrict food intake.

\section{Consideration of Future Consequences scale - food version}

In order to measure time perspective regarding eating behavior and health, participants completed the CFC-food (van Beek et al., 2013), an adapted version of the 14-item Consideration of Future Consequences Scale (CFC; Joireman et al., 2012). The CFC-food consists of two subscales: CFC-immediate and CFC-future. An example of an item of the Immediate-subscale is "I only choose my food to satisfy immediate needs, figuring the 
future will take care of itself." An example question for the Future-subscale is "I consider how my health might be in the future, and try to influence my health with my day to day eating behavior." Participants indicated their agreement with the statements on a 7-point Likert scale, ranging from 1 = "totally disagree" to 7 = "totally agree."

\section{Body Mass Index}

The height and weight of each participant was measured to calculate the Body Mass Index (BMl; kg/m²).

\section{Hunger and taste rating}

Participants were asked to indicate their current hunger at the beginning of the study. Participants were also asked to indicate how much they liked the offered snacks after removal of the bowls. Responses for hunger and taste rating were given on a $100 \mathrm{~mm}$ Visual Analogue Scale (VAS) ranging from "not at all" to "very much".

\section{Analyses}

Participants were randomly assigned to EFT $(n=23)$, food-related EFT $(n=24)$, EPT ( $n=24$ ) or food-related EPT $(n=23)$. First, group differences in the ratings of the images, restraint and BMI were explored. In case of group differences, these variables were included as a covariate on all subsequent analyses. As a manipulation check, we expected EFT images to be rated as more future-oriented than EPT images. Next, two two-way between subjects ANOVA were conducted with manipulation (EPT or EFT) and content (general or food-related images) as factors, and either discount rate or caloric intake as dependent variable. For the analysis on caloric intake, current hunger and taste rating of the snacks were included as covariates.

\section{RESULTS}

\section{Manipulation check}

Mean age of participants was 20.45 years $(S D=1.97$, range $18-29)$ and mean $B M I$ was $22.43(S D=2.75$, range $17.74-31.78)$. The conditions did not differ on age, scores on the RS or on calculated BMI (all $p$ 's > .31; see Table 1). Hunger ratings were on average intermediate $(M=53.30, S D=24.20)$ and did not differ between conditions (see Table 1). Taste ratings of the offered snacks were on average moderately high $(M=65.98, S D$ $=19.71)$. Participants who engaged in EPT $(M=70.10, S D=17.66)$ rated the snacks as more palatable than those who engaged in EFT $(M=61.87, S D=20.95), F(1,90)=4.20, p$ $<.05, \eta_{p}^{2}=.05$. For CFC-food, groups did not differ on the CFC-immediate subscale (all $p$ 's $>$.67; see Table 1). For the future subscale, a significant difference for content occurred, 
$F(1,90)=5.05, p<.05, \eta_{p}^{2}=.05$, indicating that food-related groups had higher scores on the CFC-future subscale $(M=31.95, S D=4.85)$ than general groups $(M=29.21, S D=$ 6.72).

TABLE 1 Means and standard deviations of participant characteristics per condition

\begin{tabular}{lcccc} 
& $\begin{array}{c}\text { EPT-general } \\
(\boldsymbol{n}=\mathbf{2 4})\end{array}$ & $\begin{array}{c}\text { EFT-general } \\
(\boldsymbol{n}=\mathbf{2 3})\end{array}$ & $\begin{array}{c}\text { EPT-food } \\
(\boldsymbol{n}=\mathbf{2 3})\end{array}$ & $\begin{array}{c}\text { EFT-food } \\
(\boldsymbol{n}=\mathbf{2 4})\end{array}$ \\
\hline Age & $20.21(2.55)$ & $20.17(1.47)$ & $20.41(1.65)$ & $20.79(1.86)$ \\
BMI & $22.16(2.47)$ & $22.51(3.84)$ & $22.58(2.82)$ & $22.51(2.94)$ \\
Restraint & $11.96(4.15)$ & $12.57(4.57)$ & $12.48(5.36)$ & $12.71(3.84)$ \\
CFC-food Immediate & $24.88(5.86)$ & $25.35(5.90)$ & $24.39(7.23)$ & $24.96(4.69)$ \\
CFC-food Future & $29.58(7.01)$ & $28.83(6.55)$ & $31.48(4.79)$ & $32.42(4.98)$ \\
Hunger & $52.17(22.60)$ & $51.00(22.26)$ & $47.61(30.45)$ & $62.08(19.40)$ \\
Taste rating of snacks ${ }^{a}$ & $68.18(19.93)$ & $62.64(13.73)$ & $72.10(15.13)$ & $61.13(26.39)$
\end{tabular}

Note. EPT = Episodic Past Thinking, EFT = Episodic Future Thinking, BMI = Body Mass Index, CFC-food= Consideration of Future Consequences scale - food version.

${ }^{\text {a }}$ Rated by participants on a $100 \mathrm{~mm}$ VAS, ranging from not at all (0) to very much (100).

All generated images were evaluated as reasonably realistic, detailed, vivid, easy to imagine and positive (see Table 2). No group differences on realism, details or difficulty of generating the images were found (all $p$ 's $>.24$; see Table 2). Food-related images $(M=5.97, S D=.68)$ were on average evaluated as more positive than general images $(M=5.40, S D=.92), F(1,90)=11.25, p<.01, \eta_{p}^{2}=.11$, and, although not statistically significant, as slightly more vivid $(M=5.54, S D=.61)$ than general images $(M=5.30, S D=$ .75), $F(1,90)=2.86, p=.09, \eta_{p}^{2}=.03^{1}$. Regarding the extent to which events were rated as future-oriented, a main effect of the future manipulation was found, $F(1,90)=98.70$, $p<.001, \eta_{p}^{2}=.52$, indicating that EFT groups $(M=5.23, S D=1.04)$ thought more about the future than the EPT groups $(M=2.81, S D=1.31)$. Thus, the EFT manipulation was successful in activating future images.

TABLE 2 Means and standard deviations for ratings of generated images per condition

\begin{tabular}{lcccc} 
EPT-general & $\begin{array}{c}\text { EFT-general } \\
(\boldsymbol{n}=\mathbf{2 4})\end{array}$ & $\begin{array}{c}\text { EPT-food } \\
(\boldsymbol{n}=\mathbf{2 3})\end{array}$ & $\begin{array}{c}\text { EFT-food } \\
(\boldsymbol{n}=\mathbf{2 4})\end{array}$ \\
\hline Realism & $5.60(0.77)$ & $5.61(0.93)$ & $5.79(0.68)$ & $5.73(0.75)$ \\
Details & $4.94(0.87)$ & $4.65(0.84)$ & $5.04(0.77)$ & $4.92(0.83)$ \\
Vividness & $5.41(0.72)$ & $5.19(0.78)$ & $5.55(0.59)$ & $5.52(0.64)$ \\
Difficulty & $2.91(1.09)$ & $3.00(1.18)$ & $2.96(1.08)$ & $3.10(1.47)$ \\
Positivity & $5.46(0.92)$ & $5.34(0.93)$ & $5.95(0.60)$ & $5.98(0.76)$ \\
Future-oriented & $2.91(1.25)$ & $5.46(0.80)$ & $2.71(1.40)$ & $5.01(1.20)$
\end{tabular}

Note. Images were rated by participants on a 7-point Likert scale ( $1=$ not at all; $7=$ very much). EPT = Episodic Past Thinking, EFT = Episodic Future Thinking. 


\section{Discount rate}

Groups did not differ on the vividness or amount of time spent on considering the events while they completed the MCQ (all p's > .12). In Figure 1, results of the two-way between subjects ANOVA for delay discounting are presented.' The interaction effect between manipulation and content was not significant, $F(1,90)=.01, p=.94, \eta_{p}^{2}=$ .00. There was a statistically significant main effect for the future manipulation, $F(1,90)$ $=5.66, p=.02, \eta_{p}^{2}=.06$, which indicates that EFT (general: $M=-5.63, S D=1.33$; foodrelated: $M=-5.61, S D=1.46$ ) reduced discounting of the future relative to EPT (general: $M=-5.03, S D=1.03$; food-related: $M=-4.97, S D=1.21$ ). The main effect of content, $F$ $(1,90)=.02, p=.89, \eta_{p}^{2}=.00$, was not significant, which indicates that results were not different for general or food-related images.

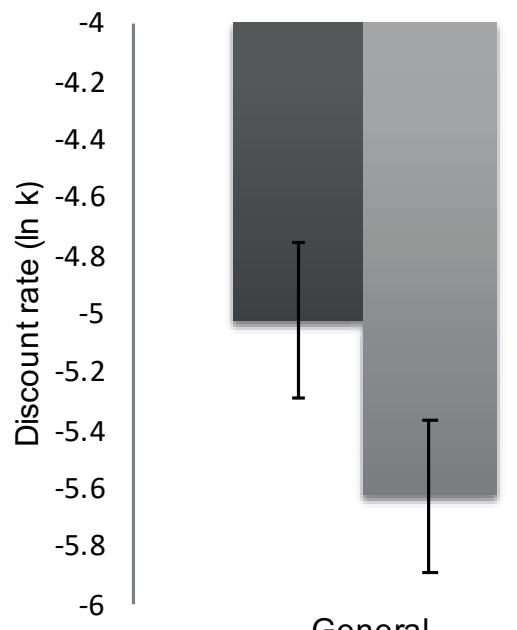

General

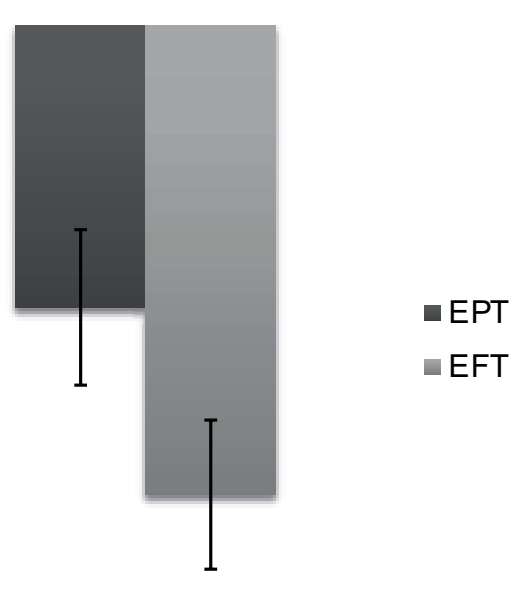

Food-specific

FIGURE 1 Means of log-transformed k-value are plotted for delay discounting. Error bars represent the standard error of the mean.

EPT $=$ Episodic Past Thinking, EFT = Episodic Future Thinking.

\section{Caloric intake}

Participants on average consumed 208.63 calories $(S D=160.56$, Range $0-616.76)$. Discount rate and caloric intake were not correlated $(r=-.03, p=.76)$. Within groups, discount rate and caloric intake were also not related (all $p$ 's $>.47$ ). Results of the twoway between-groups analysis of covariance with manipulation and content as factors, and hunger and taste ratings included as covariates are presented in Figure 2. Content of imagery was either general or food-related. Hunger, $F(1,88)=18.11, p<.001, \eta_{p}^{2}=$ .17 and taste, $F(1,88),=12.54, p=<.01, \eta_{p}^{2}=.13$, significantly predicted caloric intake. No main effect of manipulation, $F(1,88)=2.58, p=.11, \mathrm{\eta}_{p}^{2}=.03$ or content, $F(1,88)=$ 
$0.09, p=.77, \eta_{p}^{2}=.00$, occurred. The interaction between manipulation and content was significant, $F(1,88)=5.01, p=.03, \eta_{p}^{2}=.05$. Post hoc analyses performed separately for general versus food-related content revealed that food-specific EFT ( $E M M=164.46, S E$ $=29.85)$ reduced caloric intake relative to food-related EPT $(E M M=273.79, S E=30.95)$, $F(1,44)=5.97, p=.02, \eta_{p}^{2}=.12$. For general content, in contrast, caloric intake did not differ between EPT $(E M M=190.86, S E=26.78)$ and $E F T(E M M=208.10, S E=27.37), F(1$, 42) $=.20, p=.66, \eta_{p}^{2}=.01$.

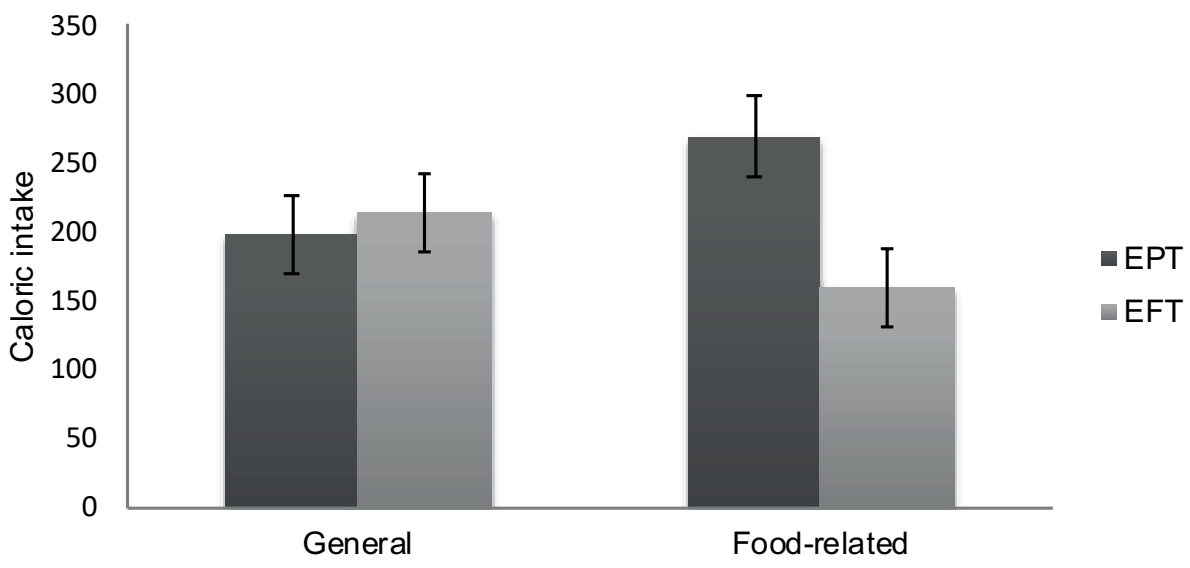

FIGURE 2 Two-way analysis of covariance controlling for the effects of hunger and taste rating of snacks. Estimated Marginal Means are plotted for caloric intake. Error bars represent the standard error of the mean.

EPT $=$ Episodic Past Thinking, EFT $=$ Episodic Future Thinking.

\section{DISCUSSION}

The aim of the current study was to test whether discount rate and caloric intake could be altered by a manipulation of episodic future thinking, by shifting concern with immediate gratification to a more future-oriented outlook. As only food-specific measures of time orientation are related to healthy eating patterns, the current study explored whether the effects of EFT on food intake could be enhanced by making the content of imagery food-related. Delay discounting was indeed reduced by EFT. This effect was not determined by content: Both general and food-related EFT led to less discounting of the future compared to control groups who relived recent episodic past events. Further, in line with our hypothesis, results showed that only food-related EFT, but not general EFT, reduced food intake.

Thus, EFT during decision making resulted in less frequent choices of immediate reward, in accordance with earlier research (Benoit et al., 2011; Daniel et al., 2015; 
Daniel et al., 2013a, 2013b; Lin \& Epstein, 2014; Peters \& Büchel, 2010). As unhealthy eaters are mainly present-minded for food and not in general (Dassen et al., 2015), only food-specific future thinking resulted in more restricted caloric intake, in line with our hypothesis. For the general manipulation, no group differences were found on caloric intake, which is surprising given earlier results (Daniel et al., 2015; Daniel et al., 2013b). It was however suggested by Daniel et al. (2015) that participants in their study may have had food-specific images come to mind, by associating positive future images with food. Hence, their general manipulation could be more closely alike to our food-specific manipulation. Importantly, our sample of participants was different to the sample of Daniel et al. (2013b, 2015), where results were found for overweight/obese women and children high in dietary restraint. Our sample consisted of female students who were not necessarily motivated to restrict their caloric intake, which could make the possibility to restrict intake of snacks and show restraint seen as irrelevant. Earlier results show that the effect of episodic thinking is related to the vividness of the imagined events: the more vivid the imagery, the greater the reduction in discount rate (Peters \& Büchel, 2010). Vividness of imagery was possibly not equal between conditions in these earlier studies (Daniel et al., 2015; Daniel et al., 2013b). As all images were rated as equal in vividness, realism, difficulty and details, the current study provided a strong control by generating episodic past events.

As stated in the introduction; we hypothesized that by changing discount rate, caloric intake would also be altered by making less impulsive food choices. However, as no association was observed between discount rate and caloric intake, it appears that this was not the mechanism underlying the effect of EFT on caloric intake. As choices where about money in the current discount task, this measure does not fully capture the real dilemma, choosing between palatable foods or future health outcomes. Though discount rate has been measured with food as a reinforcer before (Dassen et al., 2015; Odum \& Rainaud, 2003; Rasmussen et al., 2010), these measures neither capture the dilemma, because by no discounting of the future in this delay discounting tasks, the future reward would be to receive even more of the (unhealthy) food. Future delay discounting tasks mirroring this dilemma more closely could possibly clarify this. Importantly, as the reinforcing value of food has been found to interact with delay discounting in predicting BMI (Appelhans et al., 2011; Epstein et al., 2014), including a measure of the reinforcing value of food in future studies would possibly contribute to a better understanding of the variation in food consumption. Reducing discount rate may only help to prevent or intervene on obesity in those who are high in food reinforcement. In addition, the snacks provided in the current study consisted of a sample of sweet snacks, yet savory snacks are often also energy dense (de Graaf, 2006). Though data was collected on how much the participants liked the snacks, no data were 
collected on snacking preferences. As a result, findings from the present study may not be representative for participants who prefer savory snacks over candy.

Results of Cheng, Shein, and Chiou (2012) showed that the effect of a future prime on discount rate and desirability of hedonic activities was mediated by time orientation. Time orientation was measured at a behavior-specific level in the current study. The food-specific groups scored higher on the future-subscale of the CFC-food, which means that they indicated to consider future health consequences of their eating behavior to a greater extent than the general groups. Though, as no differences on the CFC-food subscales occurred between the EFT and EPT groups, time orientation seems not to be the underlying mechanism which caused changes in caloric intake. However, as CFC is considered to be a stable individual difference (Strathman et al., 1994; Toepoel, 2010), this questionnaire was probably not sensitive to pick up small changes in future orientation as a state. Thus, based on current results, the possibility that the manipulation activated a temporary focus on future health outcomes cannot be excluded. Interestingly, in the current study, for the food-specific manipulation we used a global instruction (think of an event which is related to food), which was not restricted to consider healthy or unhealthy consequences. As unhealthy eaters do not consider future health consequences (Dassen et al., 2015), tailoring the content to goals of weight loss and health benefits could turn out even more beneficial (Daniel et al., 2015). However, images should be realistic, as fantasizing has been shown to be not effective in changing behavior (Oettingen, 2012).

Importantly, based on current results, we cannot be sure whether food-related EFT reduced caloric intake, food-related EPT increased caloric intake, or both. Future research should therefore include a neutral control. In order to use EFT as an intervention, we would also like to encourage future studies to look into more detail how long time effects last, as any long term effects remain to be investigated. An interesting next step would be to investigate whether training individuals to engage in EFT would help to delay gratification in daily life.

In conclusion, the current findings indicate that impulsive behaviors can be controlled by envisioning the future vividly. Specifically, generating future images reduces impulsive decision making, whereas only the generating of food-related images influences caloric intake. Thus, it is beneficial to make the content of EFT food-related in order to change eating behavior. The underlying mechanism stays to be discovered as changes in discount rate did not directly result in more restricted caloric intake. Future studies should further explore the mechanisms underlying EFT and examine the longevity of effects on behavior. Current results propose EFT as a promising technique to change concern with immediate gratification to a more future-oriented outlook. 


\section{Funding}

This project was funded by the Maastricht University Interfaculty Program 'Eatwell'

\section{Footnotes}

1 We also conducted all analyses with positivity and vividness included as a covariate (data not shown). However, as this did not change the pattern of outcomes, results are reported for the analyses without positivity and vividness included as a covariate. 


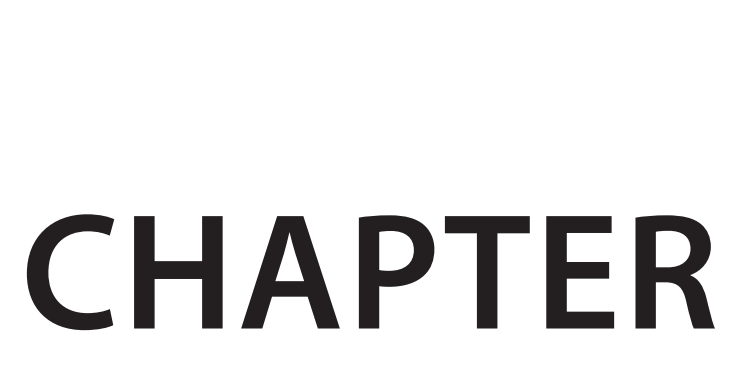

\title{
Manipulating Cognitive Flexibility:
}

Can we Change Eating Behavior through Training of Executive Control?

\author{
Dassen, F.C.M., Houben, K., Jansen, A.
}

Manipulating cognitive flexibility: Can we change eating behavior through training of executive control? (ready for submission). 


\section{ABSTRACT}

Overeating is associated with weaker shifting, one of the main executive functions. In two experimental studies we explored whether a cognitive flexibility manipulation would result in better shifting and heightened cognitive control over eating behavior. In study 1 , undergraduate females $(N=44)$ were randomly assigned to a cognitive flexibility manipulation or control. Outcome measures were the Wisconsin Card Sorting Test (WCST), Trail Making Test (TMT), virtual food choice and ad libitum food intake. In Study 2, two sessions of the manipulation or control training were completed by undergraduate females with a goal to restrict food intake $(N=80)$. Outcome measures were the WCST and TMT, ad libitum food intake and the intention-behavior gap regarding snack, fruit and vegetable intake. In study 1 , the experimental group performed marginally significant better on the WCST and TMT, whereas on virtual food choice and actual intake, no differences were found. In study 2, the experimental group performed better on the WCST, though no differences were found on TMT, food intake or the intention-behavior gap. As a limitation, the research sample consisted of undergraduates, who probably already displayed good shifting skills at baseline, and we only focussed on direct practice instead of strategy use. In conclusion, results provide no consistent evidence that our cognitive flexibility manipulation improved shifting or control over eating behavior. Future studies are encouraged to continue to study the role and trainability of cognitive flexibility in eating behavior, and to focus on strategies to improve transfer of practiced skills to daily life.

Key words: Eating Behavior; Executive Functioning; Shifting; Cognitive Flexibility Manipulation. 


\section{STUDY 1}

\section{INTRODUCTION}

Suppose you were planning to do $\mathrm{X}$, but a new opportunity arises to do Y: Are you flexible enough to take advantage of this unexpected opportunity? Shifting, also known as task switching or cognitive flexibility, is a core aspect of executive function $(E F)$, and involves being flexible enough to adjust to changed demands or priorities, and to take advantage of sudden, unexpected opportunities (Diamond, 2013; Miyake et al., 2000). EF is a set of higher level, 'supervisory' cognitive functions involved in the control and regulation of more basic, lower-level cognitive processes (Alvarez \& Emory, 2006). Influential dual-process theories such as the Reflective-Impulsive model (RIM; Hofmann et al., 2012; Strack \& Deutsch, 2004) indicate that individuals with better EF, including shifting, should be more successful in regulating their behavior in line with their long-term goals. For instance, flexible behavioral patterns might be beneficial if it helps someone to take advantage of unexpected opportunities to pursue his or her goals (Bayuk, Janiszewski, \& Leboeuf, 2010).

We know from previous research that an elevated Body Mass Index (BMI) is associated with weaker EF, including shifting (Boeka \& Lokken, 2008; Fagundo et al., 2012; Fergenbaum et al., 2009; Fitzpatrick et al., 2013; Perpiñá et al., 2016; Verdejo-García et al., 2010; Wu et al., 2014). Obesity is in essence the result of a positive energy balance, that is, when energy intake (consumption) exceeds energy expenditure. Perseverating in unhealthy eating patterns such as overeating could be viewed as being stuck in unhealthy eating habits (Voon, 2015). Adherence to a healthy diet may however be improved through flexible self-regulation by using a variety of means to accomplish this overarching goal (Hofmann et al., 2012). Previous research showed for instance that individuals with better shifting skills acted more often on their intentions to eat more fruits and vegetables and snack less than their counterparts with weaker shifting skills (Allan et al., 2011).

Thus, as shifting skills seem related to eating behavior and BMI, one potential strategy to promote a healthy eating pattern could be to improve shifting ability. Previous research has shown that EF can be improved through cognitive training (though note that wide transfer effects to daily life are still debated; e.g. Diamond \& Ling, 2016). EF training targeting inhibitory control and working memory, the two other core EF (Diamond, 2013; Miyake et al., 2000), has shown promising results in reducing food intake and promoting weight loss (e.g. Dassen et al., 2018; Houben et al., 2016; Houben \& Jansen, 2015; Lawrence et al., 2015). However, research focusing on training of shifting in the domain of eating behavior and weight loss is sparse (e.g. Raman, Hay, \& Smith, 2014). 
We developed a brief manipulation consisting of paper-and-pencil exercises inspired by Cognitive Remediation Therapy (CRT) for anorexia nervosa (Tchanturia et al., 2010). CRT, originally developed for the treatment of schizophrenia (Wykes, Huddy, Cellard, McGurk, \& Czobor, 2011), is a therapy which targets cognitive flexibility in thought processes by training shifting ability and central coherence strength (Tchanturia, 2014). The intervention combines simple cognitive exercises with reflection on thinking styles and strategies. CRT has shown promising results in the treatment of anorexia nervosa, an eating disorder which is associated with cognitive inflexibility (Tchanturia, Giombini, Leppanen, \& Kinnaird, 2017; Tchanturia et al., 2010). It has been suggested that CRT could also be adapted for the treatment of obesity (Raman et al., 2014). In the current research, we focused on the cognitive exercises that target shifting ability (Tchanturia et al., 2010). In study 1 we tested the effectiveness of this cognitive flexibility manipulation. We hypothesized that the manipulation would lead to improved performance relative to the control group on two commonly used cognitive tasks to measure shifting ability: the Wisconsin Card Sorting Test (WCST; Berg, 1948) and the Trail Making Test (TMT; Reitan, 1958). As weaker shifting and unhealthy eating behavior seem associated, we also tested whether the manipulation would influence food choice. Specifically, we expected that the experimental group would choose and consume less high-caloric foods relative to the control group.

\section{METHODS}

\section{Participants}

Forty-four female undergraduates were recruited via advertisements for a study about hunger and cognition. Participants had a mean age of 21.39 (SD $=1.72$, range 18-27) and a mean $B M I$ of 23.05 ( $S D=2.65$, range $16.70-28.43$ ). Mean restraint score of participants was $12.45(S D=5.66$, range $2-26)$.

\section{Materials and measures}

\section{Manipulation}

The cognitive flexibility manipulation was globally based on Tchanturia et al. (2010) and lasted twenty minutes. The experimental group performed the cognitive flexibility exercises, whereas control completed the same exercises without the shifting component.

Stroop Task. Participants were provided with a Stroop task (Stroop, 1935). The experimental group started the exercise with naming the ink color of the words and had to switch every twenty seconds between naming the ink color and reading the words. The control group only had to name the ink color of the words. 
Switching Attention. Participants recited the alphabet while applying two rules (e.g. alternating between providing animal names and country names), whereas the control group applied only one principle (e.g. providing animal names).

Up-and-Down Task. Participants were provided with a card displaying a staircase and arrows. They started counting the steps and continued counting backwards when they encountered an arrow pointing down, and forward when they reached an arrow pointing up. Thus, the arrows were indicators of a rule change. The control group only encountered arrows pointing up.

Embedded Words. Participants were shown word-clusters and had to select words corresponding to the instruction. The experimental group had to keep two rules in mind (e.g. crossing out words related to 'clothes' and circling words related to 'hot'), whereas the control group had the keep only one rule in mind (e.g. circling words related to 'hot').

\section{Wisconsin Card Sorting Test (WCST)}

The WCST is a commonly used shifting task (e.g. Berg, 1948; Westwood, Stahl, Mandy, \& Tchanturia, 2016). Participants had to match 128 response cards to one of four presented stimulus cards. They received feedback (i.e., 'right' or 'wrong') after every sorting. After ten consecutive correct sortings, the sorting rule changed, requiring them to shift their cognitive strategy. We used an adapted variant wherein all 128 cards were sorted (Greve, Stickle, Love, Bianchini, \& Stanford, 2005), whereas in its conventional form, the task is discontinued after six completed categories (Berg, 1948). The two most used outcomes are number of categories completed (i.e., ten consecutive correct responses) and perseverative errors (i.e., when the participants sorts the card according to the previous sorting rule) (Nyhus \& Barceló, 2009). More categories completed and fewer perseverative errors are indicative of better shifting.

\section{Trail Making Test (TMT)}

The TMT is a commonly used shifting task (e.g. Reitan, 1958; Bowie \& Harvey, 2006). In part A, participants had to connect the numbers 1-25 as fast as possible. Next, in part $B$, they had to alternate between numbers (1-12) and letters (A-L). Time to complete part $B$ minus the time to complete part $A$ was used as outcome measure, with lower switching costs indicating better shifting. The difference-score was used to isolate the shifting requirements from more general processes such as perception and responding speed (Sanchez-Cubillo et al., 2009).

\section{Food choice - virtual buffet task}

The virtual buffet task is a computer task displaying a virtual cafeteria. Participants were asked to compose an everyday lunch, by choosing from a wide array of lunch items. 
If the participant selected an item, it was added to the serving tray displayed on the screen. Participants could choose as many items as they wanted, though they were instructed to pick a lunch they would want to consume right now. Total number of kilocalories (kcal) of all chosen items was calculated.

\section{Food intake - ad libitum}

During a so-called 'break', the participant was provided with a large bowl of chocolate chip cookies (497 kcal/100 gram) and a large bowl of M\&M's (485 kcal/100 gram). As part of the cover story they no longer had to be deprived of food and were welcome to eat some snacks while completing a filler questionnaire (Five-Facet Mindfulness Questionnaire; Baer, Smith, Hopkins, Krietemeyer, \& Toney, 2006: data not analyzed). The experimenter left the room for ten minutes. Upon return, the bowls were removed and weighted in a different room, to calculate the amount of kcal consumed. The participant was asked to indicate how much she liked the provided snacks on a $100 \mathrm{~mm}$ Visual Analogue Scale (VAS). Both scores were combined into one averaged liking-score.

\section{Restraint Scale (RS)}

Participants completed the RS (Herman \& Polivy, 1980), which assesses weight concern, weight fluctuations and attempts to diet. Higher scores are indicative of an increased intention to restrict or control food intake.

\section{Body Mass Index}

The height and weight of each participant was measured with a tape-measure and a digital scale to calculate the BMI $\left(\mathrm{kg} / \mathrm{m}^{2}\right)$.

\section{Procedure}

To ensure a moderate hunger level, participants were instructed to refrain from eating two hours before participation. They were randomly assigned to the cognitive flexibility manipulation ( $n=22$ ) or the control condition $(n=22)$. They started with the cognitive flexibility or control exercises under guidance of the experimenter. Afterwards, participants indicated on a $100 \mathrm{~mm}$ VAS fun and difficulty of the exercises, craving, and energy and hunger level. They completed the virtual buffet task, TMT and WCST. Subsequently, a 'break' was introduced, in which snacks (ad libitum food intake) were provided while a filler questionnaire was completed. Afterwards participants rated the taste of the snacks, and completed the RS and demographics. Finally, participants' weight and height were measured. This study was approved by the local ethical committee. 


\section{Analyses}

One-way analyses of variance (ANOVA) were conducted to compare performance on the WCST and TMT. For the virtual buffet task, a one-way analysis of covariance (ANCOVA) was conducted with hunger included as covariate. For ad libitum food intake, an ANCOVA was conducted with hunger and liking of the snacks included as covariates (Robinson et al., 2017).

\section{RESULTS}

\section{Manipulation check and intercorrelations}

Groups did not differ on age, BMI or RS (see Table 1). The experimental group evaluated the exercises as more difficult than control. No group differences were discovered in fun of the exercises, energy level and hunger (see Table 1). The experimental group experienced marginally significant more craving. Both outcome measures of the WCST were highly correlated, $r=-.90, p<.001$. The TMT did not correlate with the outcomes of the WCST (all $p$ 's > .57). The WCST and TMT were not correlated with food choice or ad libitum food intake (all $p$ 's > .36).

TABLE 1 Means with standard deviations between parentheses displayed per group, reflecting baseline characteristics, evaluation of the exercises, hunger and craving.

\begin{tabular}{lllll} 
& Experimental & Control & $\boldsymbol{t}(\boldsymbol{d f})$ & $\boldsymbol{p}$ \\
\hline Age & $21.77(1.82)$ & $21.00(1.82)$ & $1.52(1,42)$ & .14 \\
Body Mass Index & $22.89(2.77)$ & $23.21(2.57)$ & $0.39(1,42)$ & .70 \\
Restraint Scale & $12.05(5.21)$ & $12.86(6.17)$ & $0.48(1,42)$ & .64 \\
Hunger & $55.64(26.21)$ & $45.23(26.44)$ & $1.31(1,42)$ & .20 \\
Craving & $61.18(24.82)$ & $46.73(26.80)$ & $1.86(1,42)$ & .07 \\
Difficulty & $56.50(25.15)$ & $38.77(22.01)$ & $2.49(1,42)$ & .02 \\
Fun & $54.82(25.13)$ & $55.41(17.62)$ & $0.09(1,42)$ & .93 \\
Energy level & $53.18(20.40)$ & $57.09(18.22)$ & $0.67(1,42)$ & .51
\end{tabular}

\section{Shifting}

Regarding the WCST, the experimental group completed marginally significant more categories $(M=8.05, S D=1.53)$ than the control group $(M=6.86, S D=2.62), F(1,42)=$ $3.33, p=.08, \eta_{p}^{2}=.07$. They made marginally significant fewer perseverative errors ${ }^{1}(M=$ $13.68, S D=4.55)$ than the control group $(M=18.32, S D=11.19), F(1,42)=3.24, p=.08$, $\eta_{p}^{2}=.07$. They also performed marginally significant better on the TMT $(M=11.76, S D=$ $5.90)$ than the control group $(M=15.38, S D=7.91), F(1,42)=2.96, p=.09, \eta_{p}^{2}=.07$. We explored the role of BMI, by including it as a covariate in the above analyses. However, 
BMI was no significant predictor of shifting (all $p$ 's $>.42$ ), and including BMI as a covariate did not change the pattern of results for any of the outcomes (all $p$ 's $<.10$ ).

\section{Virtual buffet task and ad libitum food intake}

In the virtual buffet task, hunger was a significant predictor, $F(1,41)=4.72, p=.04$, $\eta_{p}^{2}=.10$, indicating that more hunger was related to choosing a lunch that contained more kcal. However, groups did not differ, $F(1,41)=0.46, p=.50, \eta_{p}^{2}=.01$. The lunch of participants in the experimental group entailed on average $639.68 \mathrm{kcal}(S D=245.02)$, whereas the control group chose on average $565.09 \mathrm{kcal}(S D=211.36)$. Regarding at libitum intake, hunger was a significant predictor of intake, $F(1,40)=5.21, p=.03, \eta_{p}^{2}=$ .12 , with more hunger being related to a higher intake, whereas liking of the snacks was no significant predictor, $F(1,40)=0.72, p=.40, \eta_{p}^{2}=.02$. No differences in food intake between conditions were discovered, $F(1,40)=0.04, p=.84, \eta_{p}^{2}=.00$. Participants in the experimental group consumed on average $41.97 \mathrm{kcal}(S D=61.77)$, whereas the control group consumed on average $38.40 \mathrm{kcal}(S D=45.09)$. Adding craving as covariate, as craving differed marginally significant between groups, did not change the pattern of results. Craving was no significant predictor of virtual food choice or ad libitum intake (all $p$ 's > .46). Next, RS was explored as potential moderator. RS did not interact with condition for any of the outcomes (all $p$ 's $>.44$ ), though RS was a marginally significant predictor of food choice in the virtual buffet task $(p=.08)$, with more RS being related to less kcal chosen.

\section{DISCUSSION}

We examined whether a cognitive flexibility manipulation improved shifting ability relative to a control condition who performed the same exercises without the shifting component, and whether this improvement would translate into reduced food intake. The results seem promising, as one session of cognitive flexibility exercises improved shifting performance on the WCST and TMT; though note that results were marginally significant. On eating behavior (virtual food choice and ad libitum intake), no effects of the manipulation could be demonstrated. However, study 1 was conducted in a predominantly healthy weight student sample, and besides the RS we did not include any measures of motivation to diet. Given that shifting ability is probably most relevant when someone is attempting to change his or her eating behavior, study 2 examined the effectiveness of the cognitive flexibility manipulation in a sample of undergraduates who expressed an explicit desire to restrict their food intake with the goal to lose or maintain weight. We included a measure of shifting at baseline, to be able to correct for potential differences between conditions. In addition, we offered two training-sessions 
instead of one. As shifting ability was predictive of the extent to which individuals translated their healthy eating intentions into behavior in previous research. (e.g., the intention-behavior gap; Allan et al., 2011), we wanted to build on this and explored whether the manipulation would lead to improved adherence to intentions regarding snacks, fruits and vegetable intake during the next day. Thus, in study 2 we attempted to replicate and extend the findings of study 1, by offering a two-session cognitive flexibility manipulation to a female sample with an explicit goal to restrict intake. We hypothesized that the manipulation would improve performance on the WCST and the TMT relative to the control group, that the experimental group would consume less kcal and would choose a lower percentage of unhealthy food items in a bogus taste test, and would show a higher intention-behavior correspondence for food-consumption during the next day relative to the control group.

\section{STUDY 2}

\section{METHODS}

\section{Participants}

Eighty female undergraduates were recruited using advertisements for a study about habits and cognition. Mean age was $21.36(S D=3.53$, range 18-34) and mean BMI was 23.32 ( $S D=4.22$, range 16.69 - 36.81). Mean RS-score was 16.37 ( $S D=4.83$, range 6-28). In order to be eligible, potential participants had to answer the following question on a five-point scale: 'Are you currently trying to restrain your food intake with the goal to lose weight or maintain your body weight?' with 'probably yes' (4) or 'definitely yes' (5). Filler-questions about intentions to study and exercise were included in the screeningquestionnaire to disguise the actual goal of the study.

\section{Materials and measures}

\section{Behavioral Rating Inventory of Executive Functioning-Adult version (BRIEF-A)}

The BRIEF-A (Roth, Isquith, \& Gioia, 2005) is a self-report measure of perceived EF in daily life. The shift subscale, which assesses the ability to switch between situations as required by the circumstances, was administered at baseline (Roth et al., 2005). Scores were converted to T-values (based on a standardization sample: Roth et al., 2005) with higher T-values indicating worse self-reported shifting in daily life.

\section{Bogus taste test}

Participants were provided with four bowls containing two high-caloric foods, crisps (Lays paprika, $531 \mathrm{kcal} / 100 \mathrm{~g}$ ) and M\&M's (485 kcal/100g), and two low-caloric foods, 
baby carrots ( $33 \mathrm{kcal} / 100 \mathrm{~g}$ ) and snack tomatoes (30 kcal/100g). They were left alone with the food for twelve minutes, while they completed a taste-rating and were allowed to consume as much as they wanted. Both total amount of kcal consumed and percentage of kcal consumed from the high-caloric items were calculated as outcome measures.

\section{Intention and follow-up behavioral measurement}

Participants rated their intention to perform nine different behaviors during the next day. Included behaviors were consumption of snacks, fruits and vegetables, which were the target behaviors, and studying, watching TV, being active on social media, browsing the internet, playing videogames and exercising (globally based on Allan et al., 2011, study 1). Two days after the second session, participants received an email with a followup questionnaire in the morning, asking them to rate how often they performed the intended behaviors during the previous day. Questions were similar to the ones included in the intention-measurement. The scores for the intention to consume snacks, fruits and vegetables were subtracted from the scores of the actual behavior, to calculate the intention-behavior 'gap' with respect to food consumption (Allan et al., 2011).

\section{Procedure}

Participants completed two sessions of the cognitive flexibility manipulation scheduled within one week. The exercises were similar to the ones described in study 1 . They started the first session with completing demographics, the RS and shift subscale. Next they performed the paper-and-pencil exercises and finally they were measured and weighted. Before participation in the second session they were instructed to refrain from eating two hours before participation. The session started with the exercises, and next participants rated the exercises, indicated their hunger, energy level and craving. Subsequently, they completed the computerized WCST and paper-and-pencil TMT (in randomized order) and they performed the bogus taste test. At final, they completed the intention-measurement, and two days after participation, participants reported their performed behaviors during the previous day.

\section{Analyses}

Participants were randomly assigned to the cognitive flexibility manipulation $(n=40)$ or the control group $(n=40)$. Planned analyses were in general equal to study 1 . Regarding the WCST and TMT, we included baseline shifting as covariate. For the bogus taste test, caloric intake and percentage of unhealthy foods consumed were compared between conditions in an ANCOVA with hunger and liking included as covariates. To compare groups on the intention-behavior gap regarding snack, fruit and vegetable intake, a series of ANOVA was conducted. 


\section{RESULTS}

\section{Baseline characteristics, manipulation check and intercorrelations}

See Table 2 for an overview of baseline characteristics and evaluation of the exercises per group. Groups did not differ on RS or self-reported shifting. The experimental group was somewhat younger and had a lower BMI. Similar to study 1, the experimental group rated the exercises as somewhat more difficult than the control group. On hunger, craving, energy level and liking of the exercises no differences occurred. Participants rated the exercises in general as moderately fun.

Weaker baseline shifting was positively correlated with the RS, $r=.23, p=.04$, indicating that restrained eaters reported to be less flexible in daily life. Weaker baseline shifting also correlated with a higher intention-behavior gap for snacks, $r=.26, p=.02$, indicating that less flexibility was associated with a higher snack consumption than intended. When exploring correlations between outcome variables, total number of categories completed and perseverative errors on the WCST were highly correlated ( $r$ $=-0.81, p=<.001)$. However, similar to study 1 , both shifting tasks, the TMT and WCST, were not correlated (all $p$ 's > .46). The WCST and TMT did not show a correlation with ad libitum food intake or the intention-behavior gap for snacks, fruits or vegetables (all $p$ 's $>.22)$.

TABLE 2 Means with standard deviations between parentheses displayed per group, reflecting baseline characteristics, evaluation of the exercises, hunger and craving.

\begin{tabular}{lllll} 
& Experimental & Control & $\boldsymbol{t}(\mathbf{d f})^{a}$ & $\boldsymbol{p}$ \\
\hline Age & $20.58(2.74)$ & $22.15(4.06)$ & $2.04(1,68.34)$ & .05 \\
Body Mass Index & $22.27(3.90)$ & $24.37(4.31)$ & $2.28(1,78)$ & .03 \\
Restraint Scale & $15.83(4.01)$ & $16.70(5.65)$ & $0.80(1,70.34)$ & .43 \\
Shift subscale & $60.13(6.52)$ & $63.48(8.67)$ & $1.47(1,78)$ & .15 \\
Hunger & $57.15(27.44)$ & $57.30(26.77)$ & $0.03(1,78)$ & .98 \\
Craving & $61.73(26.95)$ & $63.48(26.88)$ & $0.10(1,78)$ & .92 \\
Difficulty & $55.88(14.33)$ & $46.53(20.88)$ & $2.34(1,69.07)$ & .02 \\
Fun & $69.40(22.62)$ & $63.48(26.88)$ & $1.07(1,75.79)$ & .29 \\
Energy level & $59.68(20.33)$ & $57.05(23.32)$ & $0.54(1,78)$ & .59 \\
a Degrees of freedom vary across t-tests depending on violation of Levene's test for equality of variances; \\
degrees of freedom were adjusted accordingly.
\end{tabular}

\section{Shifting}

For number of completed categories on the WCST, baseline shifting was a significant predictor, $F(1,77)=5.00, p=.03, \eta_{p}^{2}=.06$, indicating that weaker self-reported shifting was associated with less categories completed. More importantly, a significant 
difference between the experimental $(M=7.53, S D=2.01)$ and control group $(M$ $=6.68, S D=2.61$ ) was discovered, $F(1,77)=4.08, p<.05, \eta_{p}^{2}=.05$, indicating that more categories were completed by the experimental group than the control group. Regarding perseverative errors ${ }^{2}$, baseline shifting was no significant predictor, $F(1,77)$ $=0.84, p=.36, \eta_{p}^{2}=.01$, and no difference between the experimental group $(M=15.95$, $S D=7.13)$ and the control group $(M=18.15, S D=10.79)$ was found, $F(1,77)=1.47$, $p=.23, \eta_{p}^{2}=.02$. For the TMT, shifting was no significant predictor, $F(1,77)=1.07, p$ $=.31, \eta_{p}^{2}=.01$, and no difference between the experimental group $(M=24.08, S D=$ $13.05)$ and the control group $(M=22.72, S D=12.63)$ was discovered, $F(1,77)=0.41, p$ $=.53, \eta_{p}^{2}=.01$. As groups differed on age and BMI, we repeated the analyses with age and BMI included as covariates. BMI was a marginally significant predictor of categories completed, $F(1,75)=3.17, p=.08, \eta_{p}^{2}=.04$, indicating that a higher BMI was related to a weaker performance. When including age and BMI, the difference between groups regarding categories completed was no longer significant, $F(1,75)=2.75, p=.10, \eta^{2}$ $=.04$. BMI was a significant predictor of perseverative errors, $F(1,75)=4.15, p<.05, \eta^{2}$ $=.05$, indicating that a higher BMI was associated with less perseverative errors on the WCST. Regarding the TMT, age was a marginally significant predictor, $F(1,77)=3.72, p=$ $.06, \eta_{p}^{2}=.05$ However, for both perseverative errors on the WCST and shift costs on the TMT, including age and BMI as covariates did not change the pattern of results.

\section{Bogus taste test and intention-behavior gap}

Results of total amount of kcal consumed, with hunger and liking included as covariates, revealed hunger as a significant predictor of intake, $F(1,75)=4.50, p=.04, \eta_{p}^{2}=.06$, whereas liking of the snacks was not $(p=.50)$. No differences in food intake between groups occurred, $F(1,75)=0.78, p=.38, \eta_{p}^{2}=.01$. Participants in the experimental group consumed on average $184.32 \mathrm{kcal}(S D=127.08)$, of which $74.90 \%$ could be attributed to the unhealthy foods $(S D=19.77)$, whereas the control group consumed on average $214.70 \mathrm{kcal}(S D=166.72)$, of which $73.27 \%$ was attributable to the unhealthy foods $(S D=$ 24.19). Regarding percentage unhealthy kcal, also no differences between groups were discovered, $F(1,78)=0.11, p=.74, \eta^{2}=.01$.

See Table 3 for an overview of the intended servings, actual servings and the intention-behavior gap. No significant differences between groups were discovered. All mean intention-behavior gap scores were close to zero, indicating that participants in general succeeded in performing their intended behavior. Next, RS and BMI were explored as potential moderators of the effect of the manipulation on eating behavior. However, neither RS nor BMI did interact with condition for any of the outcome variables (all $p$ 's > .44), and none of them was a significant predictor of intake or the intentionbehavior gap (all $p$ 's $>$.12). 


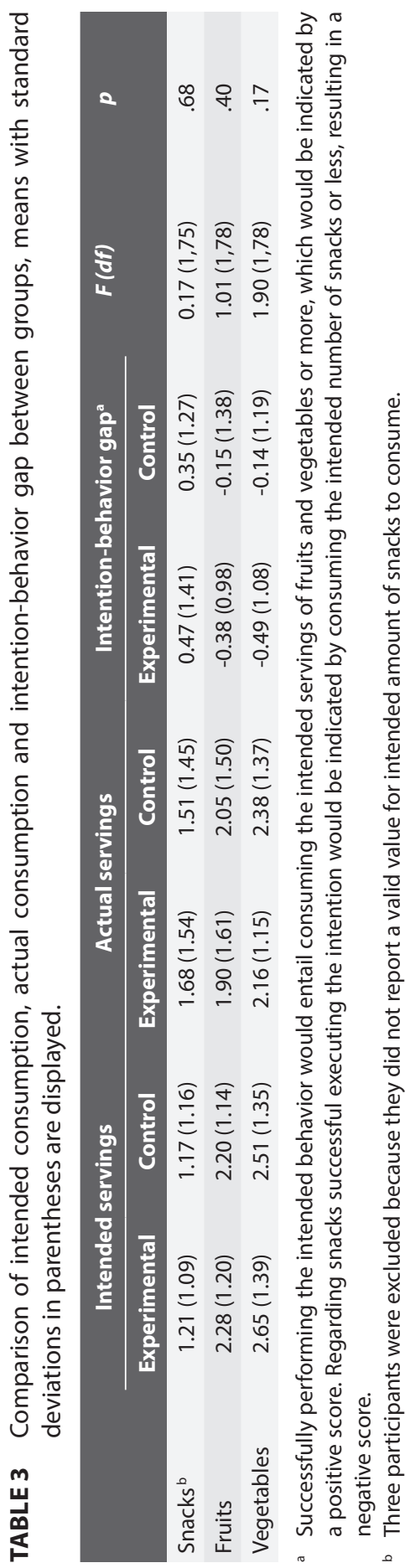




\section{GENERAL DISCUSSION}

The aim of the current research was to test whether shifting could be improved via a cognitive flexibility manipulation (study 1 and study 2), and whether this would result in reduced food intake (study 1 and study 2) and a higher intention-behavior correspondence for snack, fruit and vegetable intake (study 2), relative to a control condition. Results of study 1 revealed that shifting improved relative to the control group following the manipulation, though results were only marginally significant. Regarding eating behavior, no differences between conditions could be established. In study 2 we extended the manipulation to two sessions and explored its effects in an undergraduate female sample with an explicit goal to restrict food intake. The experimental group completed more categories on the WCST, indicating that they performed better on this shifting measure, though we could not replicate the results of study 1 for perseverative errors on the WCST or for TMT performance. Also, no effects on food intake or the intention-behavior gap were discovered. Thus, the current manipulation was neither successful in consistently improving shifting nor in changing eating behavior.

In study 1, we found a trend towards better performance on the WCST and TMT in the experimental group relative to control, and in study 2 we replicated these results for categories completed on the WCST. However, this is difficult to interpret, as results for perseverative errors on the WCST and shift costs on the TMT could not be replicated. It is possible that the initial effects found in study 1 were due to group differences in baseline characteristics. When controlling for baseline shifting in study 2 , only the effect on categories completed on the WCST could be replicated. However, these results were no longer significant after correcting for group differences in BMI and age. We did not measure performance on the exercises (practice effects), because assessing this would interfere with the manipulation itself, as this would also expose the control condition to the shifting manipulation. Therefore we have no indication about whether the experimental group performed better on the exercises than control. However, our primary aim was to examine whether practicing cognitive flexibility would improve performance on untrained tasks (e.g. the WCST and TMT) and increase cognitive control in eating behavior. This transfer-effect could not be consistently established. Earlier research provided some indications that both near (Minear \& Shah, 2008) and far transfer-effects (Karbach \& Kray, 2009) can be achieved by cognitive flexibility training, though improvements in tasks which are not directly trained are not very consistently found and highly debated (e.g. Diamond \& Ling, 2016). Thus, the transferability of switching training seems restricted to similar tasks at best, which seems in line with current results.

An important limitation of this research concerns the characteristics of the sample. Cognitive training probably has stronger effects on behavior in individuals who present 
a deficit on the EF being trained (e.g. Hofmann et al., 2012). Our sample consisted of highly educated females, which could limit the possibility to detect statistical differences between conditions because of a ceiling effect. Our manipulation did not include an adaptive component, but provided everyone with the same amount of practice time, and as a result, the manipulation could have been insufficiently cognitively challenging. In schizophrenia, applying CRT produced small to medium improvements in cognitive functioning (including performance on the TMT and WCST; Wykes et al., 2011), and there is also some evidence for improved shifting in patients with anorexia following CRT (Dahlgren \& Rø, 2014). We specifically focused on the cognitive flexibility exercises of CRT, without applying the full therapy (though note that participants were healthy undergraduates and no patients). Adding practice in strategic approaches rather than only drill and practice seems to support transfer of training effects (Wykes et al., 2011). Future studies should therefore focus on training-related features that can support generalization of learning, such as an optimal level of task difficulty (adaptive training), personal factors like mood, motivation, active interest taken in performance, variability in tasks and feedback provided (Green \& Bavelier, 2008).

We did not observe any association between shifting and eating behavior on our measures of the intention-behavior gap; only a higher intention-behavior gap for snacks was related to worse self-reported shifting ability at baseline. This is not in line with the results of Allan et al. (2011), who observed that switching/flexibility (a summary measure of three cognitive tasks including the TMT) explained $16 \%$ of the variance in the intention-behavior gap for fruit, vegetable and snack consumption. Allan et al. (2011) however measured behavior on three times a day for three consecutive days. Results for the intention-behavior gap were all close to zero in the current study, which could indicate that participants were in general successful in executing their intentions, though it is also possible that measuring behavior once resulted in an unreliable measure of the intention-behavior gap.

The lack of a direct association of shifting with eating behavior seems not that surprising, given that cognitive flexibility possibly affects behavior in a more indirect manner instead of directly leading to reduced intake. For instance, Kelly and Updegraff (2017) found an indirect effect of cognitive flexibility on heightened physical activity via activity substitution. Thus, better shifting was related to more exercising because these individuals were able to come up with alternative plans when they could not execute the original plan. Earlier results provided evidence for an association between BMI and cognitive flexibility in obese individuals, though not that consistent in healthy weight and overweight individuals (Yang et al., 2018), and this association seemed most robust when using the WCST as an outcome variable instead of the TMT (Allom, 2014). We did observe a positive association between BMI and perseverative errors on the WCST in 
our predominantly healthy weight sample, in line with results of for instance Volkow et al. (2009). However, note that our manipulation was completed before the WCST was administered, stressing careful interpretation. To shed more light on this association, future studies could explore the underlying mechanisms of the association between BMI, eating behavior and cognitive flexibility in mediation models.

In conclusion, current results do not support the effectiveness of our cognitive flexibility manipulation or its hypothesized transfer to eating behavior in healthy participants. Strong points of the current research were the control group, which only differed from the experimental condition by not including a shifting instruction, and the use of commonly used and validated outcome measures (e.g. the TMT and WCST). Given the established association between weaker shifting and obesity (Yang et al., 2018), future research could continue to study its precise role in eating behavior and its trainability in individuals with obesity.

\section{Funding}

This project was funded by the Maastricht University Interfaculty Program 'Eatwell'.

\section{ACKNOWLEDGMENTS}

We want to thank Lianne van Cleef for assisting with the data collection of study 1 and Laura Anahi Legarreta Montanez and Pauline van Gils for assisting with the data collection of Study 2.

\section{Footnotes}

1 Regarding perseverative errors, there was one outlier identified ( 3 SD from the mean) in the experimental condition. This score was on the top end of the data, and replaced by the nearest value which was not an outlier plus one.

2 Regarding perseverative errors, there was one outlier identified ( $>3 S D$ from the mean) in the control condition. This score was on the top end of the data, and replaced by the nearest value which was not an outlier plus one. 


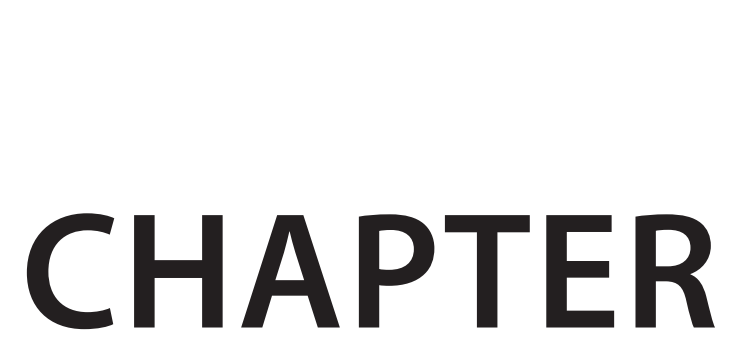

Gamified Working Memory Training in Overweight Individuals Reduces Food Intake but not Body Weight

Published as: Dassen, F.C.M., Houben, K., Van Breukelen, G.J.P., \& Jansen, A. (2018).

Gamified working memory training in overweight individuals reduces food intake but not body weight. Appetite, 124, 89-98. https://doi.org/10.1016/j. appet.2017.05.009 


\section{ABSTRACT}

Working Memory (WM) plays a crucial role in successful self-regulation of behavior, including weight regulation. Improving WM might therefore be a promising strategy to support weight loss. In the present study, overweight individuals with a desire to lose weight $(N=91)$ received an online lifestyle intervention, in conjunction with either 25 sessions of gamified WM training (experimental condition) or a sham training (control). Primary outcomes were Body Mass Index (BMI) and food intake at posttest. Secondary outcomes were executive functioning, self-control, eating style, eating psychopathology and healthy eating. Data were analyzed with mixed regression analyses with condition as between-subjects factor (experimental versus control) and time as within-subjects factor (baseline, posttest, FU1 after one month and FU2 after six months). Results revealed that the experimental condition increased their WM span more than control from pretest to posttest, and these gains were retained at FU1, though lost at FU2. No transfer effects of WM training to other executive functioning measures were found. During the bogus taste test at posttest, participants in the experimental condition consumed significantly less than participants in the control condition. However, both conditions showed a small reduction in BMI, improved eating style, reduced eating disorder pathology, and reported more self-control and a healthier eating pattern. In conclusion, the current results provide some evidence that WM training can improve eating behavior at the short term. However, the WM gains were short-lived, and the added value of WM training as an intervention to promote weight loss could not be established. Future studies should test the added value of WM training booster sessions to promote weight loss over a prolonged period of time.

Keywords: Obesity; Executive Functions; Cognitive Control; Working Memory; Weight Loss; Serious Gaming 


\section{INTRODUCTION}

Worldwide, the prevalence of overweight and obesity has increased rapidly in the past 30 years, and has currently reached epidemic proportions (Flegal et al., 2012). As a result of environmental changes in lifestyle and food supply during the last centuries, a so-called 'obesogenic environment' has emerged. Because of these environmental changes, significantly less energy is needed to deal with everyday situations, while high caloric foods are available in abundance (Chaput, Klingenberg, Astrup, \& Sjödin, 2011). If the 'energy balance', the relationship between the consumed energy and the energy used for daily activity, is positive, eventually weight will be gained (Spiegelman \& Flier, 2001). While there are numerous factors that contribute to obesity, the fundamental cause of weight gain concerns a rise in the consumption of energy-dense foods without a corresponding increase in energy expenditure (Hill \& Peters, 1998). In order to promote weight loss, behavioral interventions such as calorie-restricting diets and lifestyle interventions are frequently implemented. However, the amount of weight loss achieved via such interventions is, at best, only modest and quickly diminishes again over time (Dansinger, Tatsioni, Wong, Chung, \& Balk, 2007; Douketis, Macie, Thabane, \& Williamson, 2005). Thus, lifestyle interventions seem effective only in the short term, stressing the importance to develop new, effective weight loss interventions.

Being able to resist tempting food requires persistency to long-term health goals and not giving in to immediate pleasures. Based on dual-process theories of behavior, the ability to do this depends on the balance between two cognitive systems that control behavior: the reflective or executive system, which promotes reflective, controlled behavior, and the impulsive system, which is responsible for impulsive, automatic behavior (e.g. Hofmann, Friese, \& Strack, 2009; Strack \& Deutsch, 2004). The executive system is necessary to carry out flexible, goal-directed behavior (Hofmann et al., 2012). From a dual-process perspective, strengthening the executive system (e.g. cognitive control) could increase the capacity to act more towards the achievement of goals and standards (e.g. adherence to a diet), and provide more cognitive control over automatic impulses and behaviors that originate in the impulsive system (e.g. cravings and temptations) (Hofmann, Friese, \& Strack, 2009).

Working memory (WM) plays a central role in cognitive control (Hofmann et al., 2012). WM is one of the main executive functions (Miyake et al., 2000), and refers to the ability to temporarily store, analyze and manipulate information (Baddeley, 1992). WM is important for focused attention (Barrett et al., 2004), regulation of emotions (Schmeichel, Volokhov, \& Demaree, 2008), suppression of ruminative thoughts (Brewin \& Smart, 2005), down-regulation of cravings (Kavanagh, Andrade, \& May, 2005) and to keep mental representations of long-term goals active (Hofmann et al., 2012). Importantly, reduced WM capacity is associated with overweight and obesity (e.g. 
Gunstad et al., 2007; Smith et al., 2011), and with increased food intake, especially in combination with a strong automatic positive attitudes towards foods (e.g. Hofmann et al., 2008), while higher WM capacity is associated with increased weight loss following lifestyle intervention (Hege et al., 2013). Thus, WM seems crucial for the successful selfregulation of eating behavior and body weight.

Over the past years, several studies have shown that WM capacity can be increased through computerized training (e.g. Diamond \& Ling, 2016; Klingberg, 2010; Morrison $\&$ Chein, 2011). For instance, WM training has been found to decrease ADHD symptoms in children with ADHD (Klingberg et al., 2005), and to reduce alcohol abuse in a sample of heavy drinkers (Houben et al., 2011). In a recent study, WM training was also found to reduce pathological ruminative thoughts about food, weight, and body shape, as well as self-reported emotional eating for up to one-month after completion of the training (Houben et al., 2016). In addition, WM training reduced food intake among highly restrained eaters, though no effects were found on weight loss. However, this study did not take into account whether participants were actually motivated to put in effort to achieve weight loss, and strengthening WM capacity is probably of little use in the absence of such motivation and dieting goals (Hofmann et al., 2012; Houben et al., 2016). Also, WM training in itself is presumably not enough to support weight loss, as improved WM capacity will only be helpful if someone also has sufficient knowledge about what constitutes a healthy lifestyle and which unhealthy behaviors need to be controlled. It would, therefore, probably be more effective to provide WM training together with a lifestyle intervention, thereby directly enhancing the ability to stick to the provided dietary guidelines (Juarascio, Manasse, Espel, Kerrigan, \& Forman, 2015).

In the current study, we examined this possibility. Specifically, in the current study, overweight and obese participants received online psychoeducation about a healthy lifestyle and weight loss, in combination with either WM training or sham training. Hence, participants were provided with the necessary information to change their daily intake in order to achieve weight loss. Because online WM training can be very time consuming and monotonous for participants (Lumsden, Edwards, Lawrence, Coyle, \& Munafò, 2016), we designed the WM training as a serious game. A serious game is a computer-delivered intervention which is both enjoyable and educational (DeSmet et al., 2014). The goal of gamification is to make the boring and repetitive tasks more engaging and fun, thereby heightening motivation of participants and potentially reducing drop-out (Lumsden et al., 2016). We added a surrounding game-shell around the original WM training (Houben et al., 2016; Houben et al., 2011), thereby leaving the training-paradigm structurally intact. The scientific value of the tasks is hereby retained, though the experience and long-term engagement of participants is heightened by adding game elements. Earlier research shows that cognitive tasks can successfully 
be gamified, without losing its usefulness as a research tool (Lumsden et al., 2016). We also included rewards which became available after a completed session, which were supposed to further increase the external motivation to perform the training (Boendermaker, Prins, \& Wiers, 2015).

The primary outcomes in this study were BMI and food intake. Secondary outcomes were WM performance, self-control, eating style, eating psychopathology and healthy eating. In addition, we also assessed transfer effects of WM training to other measures of executive functions because transfer of WM training to tasks other than the trained tasks is still debated (Diamond \& Ling, 2016; Morrison \& Chein, 2011; Shipstead, Hicks, \& Engle, 2012). We examined the effect of WM training on the outcome measures immediately following training and at 1-month and 6-month follow-up. It was hypothesized that participants who received WM training would (1) show enhanced performance on a non-trained WM task, (2) show improved executive functions and self-control in daily life, (3) report less pathological thoughts regarding eating, weight and shape, (4) report less emotional and external eating, (5) consume fewer snacks in a laboratory setting at posttest, (6) report healthier eating patterns in daily life, and (7) lose more weight relative to the control condition.

\section{METHODS}

\section{Participants and design}

Participants were recruited via advertisements in local newspapers, supermarkets, and gyms, via social media, and via a general database managed by our research group. Eligibility criteria for participation were checked via a ten-minutes screening by phone by a research assistant and required that participants were aged $18-60$, were overweight (as indexed by a self-reported BMI above 25), and motivated to put in effort to achieve weight loss. Motivation was assessed via four statements which were answered on a 5-point Likert scale ranging from (1) 'totally not' to (5) 'extremely'. The items were: (1) 'How important is it for you to lose weight?', (2) ‘Do you intend to lose weight from now on?', (3) 'How determined are you to lose weight?' and (4) ‘How hard will you try to lose weight?'. In order to meet inclusion criteria, participants had to score at least 3 on all statements. The mean score of eligible participants was $4.04(S D=0.69)$. In addition, basic computer skills were checked, to make sure participants would be able to perform the online training at home. Participants were excluded if they were in treatment for an eating disorder or in the trajectory of bariatric surgery. 


\section{Materials and measures \\ Conditions: Training and control}

The current WM training was developed as a serious game, a game specifically designed to improve cognitive ability (McCallum, 2012), by adding game-elements to the original training. This training was developed by the faculty's engineering department in conjunction with the authors, and was based on the WM training-paradigm previously used by Houben et al. (2011; 2016). Participants had to perform 25 online WM sessions (experimental condition) or sham sessions (control condition) on a tablet or computer at home. All sessions and tasks were presented in an online restaurant-setting. When participants completed a session, a new item or style to decorate the restaurant became available. This gave participants the opportunity to adjust their restaurant-setting to their personal preferences. See Figure 1 for images of the gaming interface and training tasks.

To complete a full session, participants had to practice three WM tasks: a visuospatial WM task, a backward digit span task and an object memory task (see Figure 1). Participants could freely choose the order of the three tasks, consisting of 30 trials each. During the visuospatial WM task, participants monitored a sequence of flashing tables presented within a $4 \times 4$ grid, resembling a dining room. Participants had to reproduce the sequence by clicking or tapping the tables in the right order. During the backward digit span task, a sequence of numbers was shown on a memo board. Participants were instructed to reproduce the presented sequence of numbers in reversed order. During the object memory task, a kitchen cabinet with 16 closed doors was presented. Oneby-one the kitchen doors opened, revealing a kitchen-related object. Next, one of the presented objects was shown, and participants had to indicate which of the closed doors was hiding this object.

In the experimental condition, the difficulty level of the tasks was automatically adjusted on a trial-by-trial basis (adaptive WM training; Houben et al., 2016; Houben et al., 2011; Klingberg, Forssberg, \& Westerberg, 2002). Each task started with a sequence of three items. If the participant responded correctly on two consecutive trials, an item was added to the sequence on the next trial, thereby increasing task difficulty. When participants failed to reproduce the sequence correctly on two consecutive trials, the sequence on the next trial was reduced by one item, thereby decreasing task difficulty. Each new session continued at the same level with which the previous session ended. In the control condition, the difficulty level of the tasks was held constant on a basic, easy level of three items or objects for the entire training (Houben et al., 2016; Houben et al., 2011). In this way we made sure all participants practiced the tasks for an equal amount of trials, though only the experimental condition was expected to train WM by practicing at their optimal capacity limit. 
At pretest, posttest, and at follow-up, performance on the practiced training tasks (WM span) was assessed. The assessment tasks started with sequences of three items. When participants responded correctly on two consecutive trials, one item or object was added to the sequence. The task ended when participants failed to reproduce a sequence correctly on two consecutive trials. The outcome measure for each of the three WM assessment tasks was the amount of items or objects in the sequence that could correctly be reproduced. In order to calculate one total score for WM span, the three scores on the WM tasks were averaged.

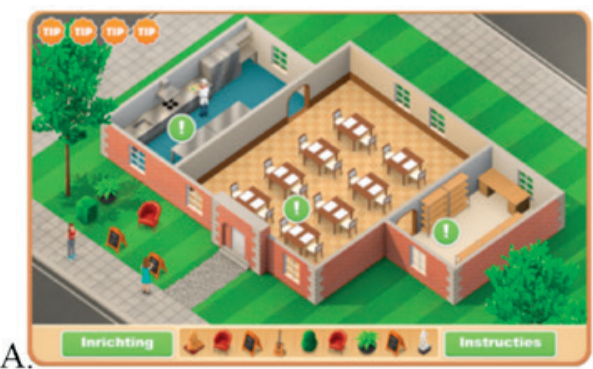

A.

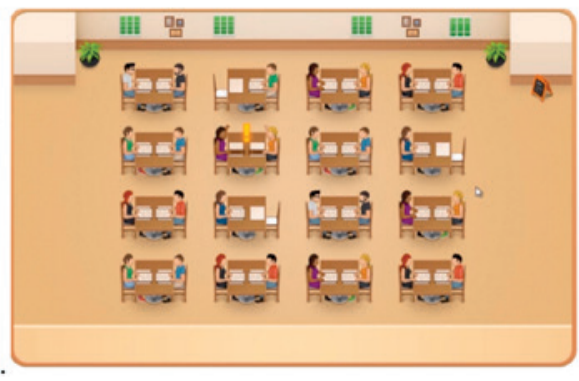

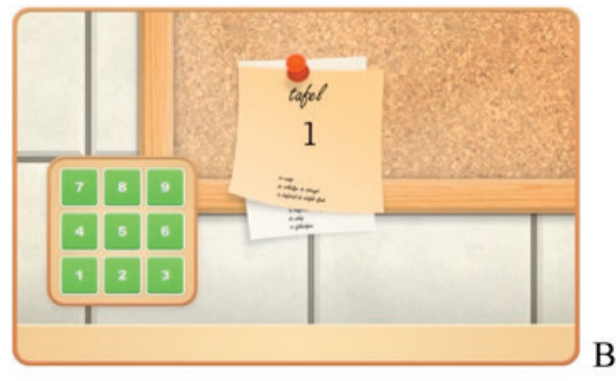

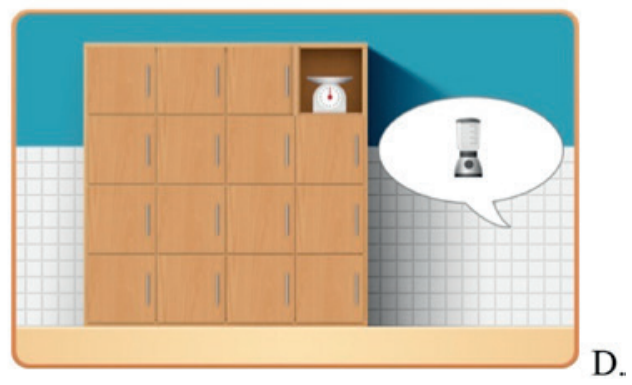

FIGURE 1 Images of the WM training: the gaming interface (A), the backward digit span task (B), the visuospatial task (C) and the object memory task (D).

\section{Lifestyle intervention}

All participants received online psychoeducation about weight loss and a healthy lifestyle, while completing the 25 sessions of WM training or sham training at home. The lifestyle intervention included four themed sessions. The first session was available at the start of the training and focused on general principles of weight loss and motivation to lose weight. Participants were advised to keep track of their daily caloric intake via an online tool. The second session addressed topics such as the 'obesogenic' environment, healthy weight loss and nutrition, and participants were encouraged to come up with their own personal diet plan. The third lifestyle session addressed several aspects of physical activity, such as the health benefits of regular physical activity and 
implementing physical activities in daily life. The fourth session discussed strategies for dealing with difficult moments and gave tips to maintain a healthy weight after the intervention. The lifestyle intervention was composed by the first author, using general nutrition information and principles of cognitive behavioral therapy (Beck, 2008). See supplementary material S1 for a more detailed description of the content of every session.

\section{2-back task}

The 2-back task is a WM task that predominantly measures the ability to monitor and update WM representations (Jaeggi, Buschkuehl, Perrig, \& Meier, 2010). The 2-back task was used to assess near transfer of WM improvement to a more complex, nontrained WM task. In the current 2-back task (based on Boselie et al., 2016), participants had to monitor a series of stimuli (i.e., letters), which were presented one-by-one on a computer screen. Participants were instructed to indicate for each letter whether the letter corresponded to the letter that was presented two stimuli before, by pressing either the letter ' $U$ ' (e.g. Yes) or ' $E$ ' (e.g. No) on the keyboard. Participants started with a practice phase, which consisted of 30 trials (10 targets and 20 non-targets). In the end, the accuracy percentage was calculated. If this was above 65 percent, participants continued immediately to the testing phase. However, if the accuracy percentage of the practice phase was below 50 percent, participants had to repeat the practice phase to make sure that they understood the task. An accuracy percentage between 50 and 65 percent gave participants the option to either repeat the practice phase or to continue to the testing phase. In the testing phase, participants completed a total of 90 trials, including 30 targets and 60 non-targets. The outcome variable of interest was accuracy, which was the summation of hits and correct rejections.

\section{Restraint Scale (RS)}

The RS (Herman \& Polivy, 1980) was assessed at baseline in order to measure the extent to which participants were trying to restrain or control their food intake. Higher scores are indicative of an increased intention to restrict food intake (Cronbach's $a=.73$ ).

\section{Bogus taste test}

At posttest, participants' food intake was measured with a bogus taste test. During the taste test, participants were presented with four bowls containing energy-dense palatable foods and a food-rating form. During the taste test, participants were left alone in the room while the experimenter waited in an adjacent room. The bowls were filled with two different types of salted potato chips (Lays, $541 \mathrm{kcal} / 100 \mathrm{~g}$; AH Excellent, $515 \mathrm{kcal} / 100 \mathrm{~g}$ ) and two different types of milk chocolate buttons (Callebout Select, 563 
$\mathrm{kcal} / 100 \mathrm{~g} ;$ Aldi, $560 \mathrm{kcal} / 100 \mathrm{~g}$ ). Participants were instructed to consume as much or as little as they preferred in order to complete the food ratings. On the food rating form, participants first indicated for how many hours they had not eaten anything, and to what extent they experienced hunger, desire and craving at that moment on a 100 millimeter Visual Analogue Scale (VAS) ranging from (0) 'not hungry/no desire/no craving' to (100) 'very hungry/strong desire/strong craving'. Next, participants had to indicate for each food item how much they liked the food on a VAS ranging from (0) 'not at all' to (100) 'very much liked'. Finally, participants compared and rated the different types of food on various taste dimensions (e.g. 'which product tastes sweeter?'). After 10 minutes, the experimenter returned and removed the bowls of food. Each bowl was weighted before and after the taste test and grams consumed were converted to calories to calculate the total amount of calories consumed.

\section{Behavioral Rating Inventory of Executive Functioning - Adult version (BRIEF-A)}

The BRIEF-A (Roth, Isquith, \& Gioia, 2005) is a 75 item self-report questionnaire to assess executive functions in daily environment. The BRIEF-A consists of nine subscales: inhibit, shift, emotional control, self-monitor, initiate, working memory, plan/organize, organization of materials, and task monitor (Hocking, Reeve, \& Porter, 2014). The nine subscales can be combined in one Global Executive Composite (GEC) score. Participants had to indicate for each item whether the statement applied to them on a 3-point Likert scale, with the options 'never', 'sometimes' or 'always'. An example item of the working memory subscale is 'I have trouble with assignments or tasks that require more than one step.' T-values (standardized scores with $M=50$ and $S D=10$, based on a general normative sample: $\mathrm{T} \geq 65$ = clinically significant) were calculated with higher T-values reflecting a greater degree of executive dysfunction. In the current study, T-values for the GEC (Cronbach's $a=.96$ ) and the WM subscale were the variables of interest (Cronbach's $a=.85)$.

\section{Brief Self-Control Scale (Brief SCS)}

The brief 13-item SCS was utilized to assess general self-control (Tangney, Baumeister, \& Boone, 2004). On each item, participants had to indicate to what extent they agreed with the statement on a 5-point Likert scale ranging from 'not at all like me' (1) to 'very much like me'(5). Example items are'I am good at resisting temptation' and 'sometimes I can't stop myself from doing something, even if I know it is wrong'. Higher scores on the SCS indicate higher levels of self-control (Cronbach's $a=.81$ ). 


\section{Dutch Eating Behavior Questionnaire (DEBQ)}

The DEBQ (Van Strien, Frijters, Bergers, \& Defares, 1986) is a 33-item self-report measure with three subscales: emotional eating, external eating and restrained eating. Items are scored on a 5-point Likert scale, ranging from 'never' (1) to 'very often' (5). Means were calculated for only the emotional and external eating subscale, with higher scores indicating increased emotional eating (Cronbach's a $=.95$ ) or external eating (Cronbach's $a=.84)$.

\section{Eating Disorder Examination - Questionnaire 6.0 (EDE-Q)}

The EDE-Q 6.0 (Fairburn \& Beglin, 2008) is a 28-item self-report measure of eating disorder pathology, in particular eating, weight and shape concerns. The EDE-Q contains 22 items assessing the frequency and severity of eating disorder psychopathology over the previous 28 days, which are answered on a 7-point Likert scale, ranging from 'not one day' (0) to 'every day' (6) or'not at all (0) to'markedly' (6). An example item is:'On how many of the last 28 days did you had a clear wish to have a completely flat stomach?' Higher total EDE-Q scores are indicative of stronger eating disorder psychopathology (Cronbach's $\alpha=.89$ ).

\section{Healthy eating behavior}

In order to determine healthy eating behavior in daily life, participants completed a 5 -item self-report questionnaire about their eating behavior during the past two weeks (Baker et al., 2003; Dassen et al., 2015; Kuijer \& Boyce, 2012, 2014). The items focused on how often someone (1) ate appropriate portions of food (not too much or too little), (2) ate in a balanced way, with a lot of fruits and vegetables, (3) ate junk food (potato chips, desserts, sweets, candy bars, etc.), (4) overate (kept eating while already satiated), and (5) ate breakfast. Items were answered on a 5-point Likert scale, ranging from 'every day' (1) to 'less than once a week' (5). A total score for healthy eating was calculated (Cronbach's $a=.65$ ), with higher scores indicating healthier eating behavior during the past two weeks.

\section{Body Mass Index (BMI) and percentage BMI-loss}

A digital scale was used to assess the weight of the participants and a measuring stick was used to assess their height. BMI was calculated $\left(\mathrm{kg} / \mathrm{m}^{2}\right)$ as well as percentage BMIloss relative to baseline [(BMI pre - BMI post) / BMI pre] x 100). 


\section{Procedure}

All participants were tested individually. Upon arrival, participants were seated behind a computer and the researcher created an online account after which the tasks and questionnaires were completed (see Supplementary material S2 for an overview of all tasks and questionnaires provided in order of administration). Next, participants were asked to remove shoes and any heavy clothing (e.g. jacket) to measure height and weight. The next day, participants started the 25 sessions of online training at home, one session a day. Participants received daily reminders via email. The training was flexible by allowing variable time intervals between sessions: participants were required to perform a minimum of 20 training sessions and a maximum of 25 training sessions, with a minimum interval of $24 \mathrm{~h}$ and a maximum interval of $48 \mathrm{~h}$ between sessions. If participants missed more than five sessions, they dropped out of the study.

After finishing the first, fifth, 10th and 15th WM session, a new lifestyle-session became available in the online training program. The lifestyle-sessions remained accessible during the training. The posttest was scheduled after completion of the training. There was an average of 41.58 days between baseline and posttest ( $S D=$ 9.48). The first follow-up measurement (FU1) was scheduled one month $(M=31.26$ days, $S D=8.32$ ) after completion of the training and the second follow-up (FU2) was scheduled six months $(M=167.48$ days, $S D=10.96)$ after completion of the training. Participants received gift vouchers of $€ 20$ following completion of the posttest, FU1 and FU2, respectively, resulting in a total remuneration of $€ 60$ for full participation. This study was approved by the Ethical Review Committee of the Faculty of Psychology and Neuroscience (ERCPN) of Maastricht University.

\section{Statistical analyses}

The primary analysis was intention-to-treat and included all participants who were randomized. Data were analyzed with mixed regression analysis using maximum likelihood estimation and an unstructured covariance matrix for the repeated outcome measures. Our model was a marginal model with repeated measures nested within persons. Condition was a between-subjects factor ( $1=$ training versus $0=$ control) and time a within-subjects factor (baseline, posttest, FU1 and FU2, with baseline as reference category and a dummy indicator per other time point). Age, sex (with male as reference category) and education level (with low education level as reference category and dummy indicators for medium and high education level) were included as covariate in all models. Likelihood ratio testing of the covariate by time interaction (with $d f=3$ for age*time and sex*time and $d f=6$ for education*time) was performed to check for significant interactions. The covariate by time interactions were removed from the model if not significant. The effect of training was examined by testing the 
interaction between time and condition, since no group difference was expected at pretest due to randomization. The final mixed model for each outcome was rerun with restricted maximum likelihood estimation to obtain unbiased standard errors (Verbeke \& Molenberghs, 2000). For the bogus taste test at posttest, caloric intake in the experimental condition was compared with intake in the control condition using ANCOVA, with age, sex, education level and liking of the food included as covariate.

As a manipulation check, the performance on the assessment-version of the trained tasks (mean WM span) was compared between training and control. Primary outcome measures were BMI (all time points) and snacking behavior (during a bogus taste test at posttest). Secondary outcome measures were accuracy at the 2-back task, self-reported executive functioning, self-control (Brief SCS), eating style (external and emotional eating: $D E B Q)$, eating psychopathology (EDE-Q), and healthy eating during the past two weeks. All analyses were two-sided, with an a of .05 for primary outcomes and an adjusted a of .01 for secondary outcomes to correct for multiple testing.

\section{RESULTS}

\section{Baseline}

A total of 91 participants (74.7\% female) met the eligibility criteria, completed the baseline measurement and were randomly assigned to one of two conditions: training ( $n$ $=51$ ) or control $(n=40)$. Of this sample of participants, $8.8 \%$ received primary education, $38.5 \%$ received secondary education and $52.7 \%$ received higher education. Mean BMI of participants was $30.76(S D=3.77)$, and the mean RS score was 15.61 (SD = 4.51). The mean age of the sample was 47.97 years $(S D=10.69)$ (See $S 3$ in the supplementary material for an overview of all baseline characteristics per condition. None of the differences was significant, all $p$ 's $>.08$, but such baseline tests are not recommended for RCT's, see Moher et al., 2010).

\section{Dropout and descriptives at posttest and follow-up}

A total of 21 participants dropped out ( 5 men, 16 women) from pre- to posttest, due to personal circumstances or because they missed too many training sessions ${ }^{2}$. In total, 70 participants ( 18 men, 52 women) completed at least 20 training sessions and the posttest. One participant dropped out from posttest to FU1 and two additional participants dropped out from FU1 to FU2, resulting in a total of 67 participants (experimental condition: $n=34$; control condition: $n=33$ ) who completed all measurements ${ }^{3}$. In Table 1 observed means (with $S D$ and $n$ ) per condition per time point are displayed for BMI and secondary outcomes. All randomized participants were included in the outcome analyses with all their available data using maximum likelihood estimation, which is a 
good method to handle missing outcome data (see Verbeke and Molenberghs, 2000, for details).

TABLE 1 Observed means for Body Mass Index $\left(\mathrm{kg} / \mathrm{m}^{2}\right)$ and secondary outcomes, displayed per condition per time point.

\begin{tabular}{|c|c|c|c|c|c|c|c|c|}
\hline & \multicolumn{4}{|c|}{ Experimental condition } & \multicolumn{4}{|c|}{ Control condition } \\
\hline & $\begin{array}{c}\text { Baseline } \\
\text { (SD) } \\
n=51\end{array}$ & $\begin{array}{c}\text { Posttest } \\
\text { (SD) } \\
n=34\end{array}$ & $\begin{array}{c}\text { FU1 } \\
\text { (SD) } \\
n=34\end{array}$ & $\begin{array}{c}\text { FU2 } \\
\text { (SD) } \\
n=34\end{array}$ & $\begin{array}{c}\text { Baseline } \\
\text { (SD) } \\
n=40\end{array}$ & $\begin{array}{c}\text { Posttest } \\
\text { (SD) } \\
n=36\end{array}$ & $\begin{array}{c}\text { FU1 } \\
\text { (SD) } \\
n=35\end{array}$ & $\begin{array}{c}\text { FU2 } \\
\text { (SD) } \\
n=33\end{array}$ \\
\hline BMI & $\begin{array}{l}30.96 \\
(3.64)\end{array}$ & $\begin{array}{l}29.95 \\
(3.46)\end{array}$ & $\begin{array}{l}29.78 \\
(3.56)\end{array}$ & $\begin{array}{l}29.65 \\
(3.80)\end{array}$ & $\begin{array}{l}30.49 \\
(3.97)\end{array}$ & $\begin{array}{l}30.17 \\
(4.14)\end{array}$ & $\begin{array}{l}30.28 \\
(4.31)\end{array}$ & $\begin{array}{l}30.34 \\
(4.55)\end{array}$ \\
\hline 2-back task ${ }^{13}$ & $\begin{array}{c}73.71 \\
(12.22)\end{array}$ & $\begin{array}{l}78.03 \\
(9.42)\end{array}$ & - & - & $\begin{array}{l}75.52 \\
(8.18)\end{array}$ & $\begin{array}{c}75.12 \\
(14.07)\end{array}$ & - & - \\
\hline BRIEF-A: WM subscale & $\begin{array}{c}60.27 \\
(10.52)\end{array}$ & $\begin{array}{c}59.85 \\
(12.43)\end{array}$ & $\begin{array}{c}58.21 \\
(12.74)\end{array}$ & $\begin{array}{c}57.94 \\
(13.06)\end{array}$ & $\begin{array}{c}56.33 \\
(10.16)\end{array}$ & $\begin{array}{c}56.94 \\
(10.87)\end{array}$ & $\begin{array}{c}54.09 \\
(11.56)\end{array}$ & $\begin{array}{l}53.03 \\
(9.90)\end{array}$ \\
\hline BRIEF-A: GEC & $\begin{array}{l}56.47 \\
(9.76)\end{array}$ & $\begin{array}{c}57.38 \\
(10.42)\end{array}$ & $\begin{array}{c}55.03 \\
(10.29)\end{array}$ & $\begin{array}{c}55.47 \\
(11.31)\end{array}$ & $\begin{array}{c}51.90 \\
(10.89)\end{array}$ & $\begin{array}{c}51.94 \\
(10.25)\end{array}$ & $\begin{array}{c}49.83 \\
(11.19)\end{array}$ & $\begin{array}{l}49.64 \\
(9.93)\end{array}$ \\
\hline Brief SCS & $\begin{array}{c}2.97 \\
(0.62)\end{array}$ & $\begin{array}{c}3.08 \\
(0.69)\end{array}$ & $\begin{array}{c}3.28 \\
(0.65)\end{array}$ & $\begin{array}{c}3.17 \\
(0.65)\end{array}$ & $\begin{array}{c}3.23 \\
(0.57)\end{array}$ & $\begin{array}{c}3.29 \\
(0.63)\end{array}$ & $\begin{array}{c}3.36 \\
(0.65)\end{array}$ & $\begin{array}{c}3.39 \\
(0.64)\end{array}$ \\
\hline External eating & $\begin{array}{c}3.09 \\
(0.61)\end{array}$ & $\begin{array}{c}3.00 \\
(0.58)\end{array}$ & $\begin{array}{c}2.87 \\
(0.56)\end{array}$ & $\begin{array}{c}2.88 \\
(0.58)\end{array}$ & $\begin{array}{c}3.04 \\
(0.70)\end{array}$ & $\begin{array}{c}2.80 \\
(0.64)\end{array}$ & $\begin{array}{c}2.72 \\
(0.60)\end{array}$ & $\begin{array}{c}2.65 \\
(0.67)\end{array}$ \\
\hline Emotional eating & $\begin{array}{c}2.77 \\
(0.84)\end{array}$ & $\begin{array}{c}2.58 \\
(0.78)\end{array}$ & $\begin{array}{c}2.45 \\
(0.80)\end{array}$ & $\begin{array}{c}2.52 \\
(0.91)\end{array}$ & $\begin{array}{c}2.69 \\
(0.86)\end{array}$ & $\begin{array}{c}2.53 \\
(0.90)\end{array}$ & $\begin{array}{c}2.38 \\
(0.87)\end{array}$ & $\begin{array}{c}2.43 \\
(0.92)\end{array}$ \\
\hline EDE-Q & $\begin{array}{c}2.56 \\
(0.78)\end{array}$ & $\begin{array}{c}2.24 \\
(0.67)\end{array}$ & $\begin{array}{c}1.94 \\
(0.91)\end{array}$ & $\begin{array}{c}1.93 \\
(0.95)\end{array}$ & $\begin{array}{c}2.30 \\
(0.86)\end{array}$ & $\begin{array}{c}1.98 \\
(0.62)\end{array}$ & $\begin{array}{c}1.73 \\
(0.94)\end{array}$ & $\begin{array}{c}1.61 \\
(0.89)\end{array}$ \\
\hline Healthy eating & $\begin{array}{l}18.88 \\
(3.44)\end{array}$ & $\begin{array}{l}20.15 \\
(2.80)\end{array}$ & $\begin{array}{l}20.74 \\
(3.01)\end{array}$ & $\begin{array}{l}20.56 \\
(2.31)\end{array}$ & $\begin{array}{l}18.90 \\
(3.43)\end{array}$ & $\begin{array}{l}20.53 \\
(2.47)\end{array}$ & $\begin{array}{l}20.17 \\
(3.10)\end{array}$ & $\begin{array}{l}20.15 \\
(2.96)\end{array}$ \\
\hline
\end{tabular}

Note. $N$ varies per measurement due to dropout or missing data, which may bias observed means, see Table 2 and supplementary material for bias adjusted effects and means.

$\mathrm{BMI}=$ Body mass Index, BRIEF = Behavioral Rating Inventory of Executive Functioning, WM = Working Memory subscale, GEC = General Executive Functioning, SCS = Self-Control Scale, EDE-Q = Eating Disorders Examination - Questionnaire.

\section{Manipulation check}

Completion of one online WM session took on average 25.71 minutes (SD = 31.56) for the control condition and 38.44 minutes $(S D=25.51)$ for the experimental condition 4 . The control condition missed on average 0.92 sessions $(S D=1.52)$ and the experimental condition missed on average 1.85 sessions $(S D=1.73)$. The total number of completed sessions varied between 20 and $25(M=23.63, S D=1.68)$. The training was on average completed within 33.57 days $(S D=7.98)$. The control condition practiced every day at the same level of three items, whereas the experimental condition showed a steady increase in number of items that could be recalled during the online training at home (see supplementary material S4 for an overview of the number of items that could be recalled daily during the 25 sessions of the online training). 
The performance on the assessment-version of the trained tasks (WM span) after completion of the training was compared between training and control by testing the interaction between time and condition. Age, sex and education level were included as covariates. None of the interactions of covariate by time were significant, and therefore not included in the final model. Likelihood ratio testing of the group by time interaction (i.e., comparing the -2loglikelihood of the model with versus without the interaction, see Verbeke \& Molenberghs, 2000) showed significant interaction, with $X^{2}(d f=3)=$ $16.94, p=<0.01$. More specifically, in the control group the score increased by about 1 from pretest to posttest and hardly changed from there on. In the experimental group the score increased by 2 from pretest to posttest and FU1, and then dropped to the level of the control group at FU2. See Figure 2 for a graphical display of results.

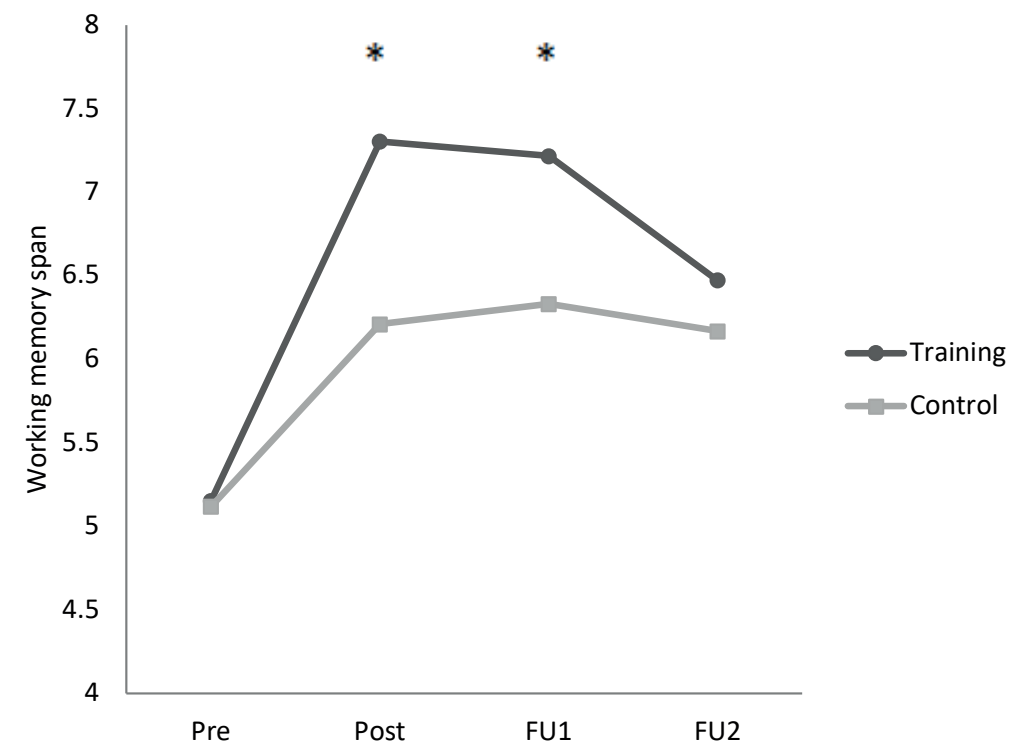

FIGURE 2 Estimated means of working memory span (number of items of trained tasks that could be correctly recalled at pretest, posttest, follow-up 1 and follow-up 2) based on the final model (REML). The average of the three trained tasks (working memory span) is displayed.

Note. ${ }^{*}$ indicates significant differences at $p<.05$.

\section{Bogus taste test}

Seventy participants completed the bogus taste test at posttest due to dropout during the training. One participant could not perform the taste test because of medical dietary restrictions, and was therefore excluded from this analysis, resulting in a sample of 69 participants (experimental: $n=34$; control: $n=35$ ). Caloric intake ranged from $5.72 \mathrm{kcal}$ 
to $295.90 \mathrm{kcal}$. Training and control condition did not differ on hunger, desire, craving, or liking of the snacks (all $p$ 's $>$.69). On average, participants rated their liking of the food with a score of 51.29 on a scale of $0-100$ (SD = 18.03, range: 16.25-100). A one-way ANCOVA was conducted to compare food intake during the bogus taste test between training and control, with age, sex, education level and participants' liking of the food included as a covariate. Results revealed liking as a significant predictor of caloric intake, $F(1,63)=6.20, p=.02$. None of the other covariates were significant predictors of intake (all $p$ 's > .50). Importantly, a significant difference between the two conditions on food intake was found, $F(1,63)=4.17, p<.05,95 \% \mathrm{Cl}$ for mean difference $[-65.45,-0.71]$. This indicates that participants in the experimental condition (estimated marginal mean = $72.86, S E=11.41$ ) consumed significantly less calories than participants in the control condition (estimated marginal mean $=105.94, S E=11.25$ ) during the bogus taste test.

We explored post-hoc potential moderators of this effect. Dietary restraint (RS) at baseline and craving were first standardized and entered as continuous predictor in the ANCOVA. The interaction between RS and condition did not reach significance, $F(1,61)=0.26, p=.61$.The interaction between craving and condition did reach significance, $F(1,61)=7.62, p<.01$. Next, we analyzed the effect of the training separately for participants with high versus low craving (respectively 1 SD above and 1 SD below the mean score; Cohen et al., 2003). For participants with low craving (-1 SD), there was no significant difference between training and control with respect to food intake (mean difference 9.96, 95\% Cl [-32.45 - 51.47], $F(1,61)=1.22, p=.64)$. For participants with high craving (+1SD), food intake was higher in the control condition compared to the experimental condition (mean difference $72.89,95 \% \mathrm{Cl}[30.04-115.74], F(1$, 61) $=11.57, p=.001$.

\section{Body Mass Index}

None of the interactions of covariate by time were significant, and these were therefore not included in the final mixed regression model. Within this final model consisting of covariate main effects, condition, time and condition by time interaction, the latter interaction was not significant either (LR test: $\mathrm{X}^{2}(d f=3)=3.52, p=.32$; see Table 2 ). This indicates that there were no differences in weight loss between conditions (see S6 and S7 in the supplementary material for more details). The non-significant interaction between time and condition was next removed from the model, to test the main effect of time, showing a very small but significant decrease of BMI from pretest to all other time points (see Table 3). On the average, participants lost a total of respectively $1.26 \%$ of their BMI at posttest, $1.52 \%$ at FU1 and $1.61 \%$ at FU2 relatively to baseline. The main effect of condition was not significant $(p>.47)$, as expected given the randomized assignment and absence of condition by time interaction. We explored post-hoc dietary 
restraint (RS) as potential moderator for BMI. However, within the model consisting of covariate main effects, condition, RS, time, time by condition interaction, RS by condition interaction, time by RS interaction, and the three-way interaction between RS, time and condition, the latter interaction was not significant (LR test: $\mathrm{X}^{2}(d f=3)=0.79, p=.85$ ).

TABLE 2 Condition by time effects on Body Mass Index $\left(\mathrm{kg} / \mathrm{m}^{2}\right)$ and secondary outcomes, established with mixed linear regression $(N=91)$.

\begin{tabular}{lcccc} 
& $\begin{array}{c}\text { Condition } \\
\text { (SE) }\end{array}$ & $\begin{array}{c}\text { Condition*posttest } \\
\text { (SE) }\end{array}$ & $\begin{array}{c}\text { Condition*FU1 } \\
\text { (SE) }\end{array}$ & $\begin{array}{c}\text { Condition*FU2 } \\
\text { (SE) }\end{array}$ \\
\hline BMI & $0.43(0.84)$ & $0.08(0.18)$ & $-0.09(0.24)$ & $-0.24(0.36)$ \\
2-back task' & $-1.55(2.31)$ & $3.10(2.00)$ & - & - \\
BRIEF-A: WM subscale & $4.04(2.25)$ & $-2.33(1.37)$ & $-1.71(1.45)$ & $-1.02(1.68)$ \\
BRIEF-A: GEC & $3.53(2.21)$ & $0.38(1.02)$ & $-0.52(1.22)$ & $-0.04(1.57)$ \\
Brief SCS & $-0.20(0.13)$ & $0.02(0.07)$ & $0.18(0.09)$ & $0.06(0.08)$ \\
External eating & $0.00(0.14)$ & $0.01(0.08)$ & $-0.03(0.09)$ & $-0.01(0.11)$ \\
Emotional eating & $0.06(0.17)$ & $-0.20(0.12)$ & $-0.20(0.13)$ & $-0.18(0.16)$ \\
EDE-Q & $0.24(0.16)$ & $-0.05(0.11)$ & $-0.12(0.16)$ & $-0.02(0.18)$ \\
Healthy eating & $0.09(0.76)$ & $-0.41(0.69)$ & $0.50(0.77)$ & $0.45(0.76)$
\end{tabular}

Note. The final mixed model (REML estimation) included the following predictors: condition, time and condition*time, correcting for age, sex and education level. The 'main' condition effect is the group difference at pretest, given that the mixed model includes the condition by time interaction terms and that pretest is the reference category for the dummy coding of time. The condition*time effect is the group difference with respect to the change from baseline to this time point.

$\mathrm{BMI}=$ Body mass Index, BRIEF $=$ Behavioral Rating Inventory of Executive Functioning, $\mathrm{WM}=$ Working Memory subscale, GEC = General Executive Functioning, SCS = Self-Control Scale, EDE-Q = Eating Disorders Examination - Questionnaire.

\section{Secondary outcomes}

None of the interactions of covariate by time were significant, and these were therefore not included in the final model. Within that model, the condition by time interaction was not significant either (all $p$ 's $>0.10$ for all secondary outcomes according to the LR test of the model with versus without interaction, remember that $\alpha=.01$ for secondary outcomes in view of multiple testing; see Table 2 for more details). The models were rerun with only condition and time as predictors included, corrected for age, sex and education level, to test for possible main effects of time, relative to baseline. See Table 3 for an overview of the main results. The main condition effect was never significant in these models (all p's > .07). Participants showed improvement on the 2-back task at posttest. They reported improved WM in daily life one month after finishing the training relative to baseline, and effects were retained at FU2. Participants also indicated improved self-control at FU1 and FU2. Regarding eating style, self-reported emotional eating was decreased at FU1 and FU2, but not directly after the training at posttest. 
Participants reported less external eating at posttest, at FU1 and at FU2. Pathological thoughts regarding eating, weight and shape were decreased at posttest, and improvements were retained at FU1and FU2. At the same time, participants indicated to eat healthier at posttest, FU1 and FU2.

TABLE 3 Main effects of time on Body Mass Index $\left(\mathrm{kg} / \mathrm{m}^{2}\right)$ and secondary outcomes, established with mixed linear regression without condition by time effect in the model $(N=91)$.

\begin{tabular}{lcccc} 
& Condition (SE) & Posttest (SE) & FU1 (SE) & FU2 (SE) \\
\hline BMI & $0.60(0.83)$ & $-0.37(0.09)^{* *}$ & $-0.43(0.12)^{* *}$ & $-0.48(0.18)^{*}$ \\
2-back task' & $0.14(2.04)$ & $2.68(1.01)^{* *}$ & - & - \\
BRIEF-A: WM subscale & $3.95(2.23)$ & $-0.41(-0.70)$ & $-2.46(0.73)^{* *}$ & $-2.94(-0.84)^{* *}$ \\
BRIEF-A: GEC & $3.89(2.13)$ & $0.48(0.50)$ & $-1.45(0.61)$ & $-1.19(0.78)$ \\
Brief SCS & $-0.17(0.13)$ & $0.05(0.04)$ & $0.17(0.04)^{* *}$ & $0.11(0.04)^{* *}$ \\
External eating & $-0.01(0.13)$ & $-0.15(0.04)^{* *}$ & $-0.25(0.04)^{* *}$ & $-0.26(0.05)^{* *}$ \\
Emotional eating & $-0.02(0.16)$ & $-0.16(0.06)$ & $-0.29(0.06)^{* *}$ & $-0.23(0.08)^{* *}$ \\
EDE-Q & $0.21(0.14)$ & $-0.30(0.06)^{* *}$ & $-0.57(0.08)^{* *}$ & $-0.63(0.09)^{* *}$ \\
Healthy eating & $0.20(0.52)$ & $-1.28(0.34)^{* *}$ & $-1.41(0.39)^{* *}$ & $-1.28(0.38)^{* * *}$
\end{tabular}

Note. The final mixed model (REML estimation) included the following predictors: condition and time (only main effects), correcting for age, sex and education level. The condition effect is the group difference average over all time points. Posttest, FU1 and FU2 display the average change (averaged over conditions) from baseline to that time point.

$\mathrm{BMI}=$ Body mass Index, BRIEF $=$ Behavioral Rating Inventory of Executive Functioning, $\mathrm{WM}=$ Working Memory subscale, GEC = General Executive Functioning, SCS = Self-Control Scale, EDE-Q = Eating Disorders Examination - Questionnaire.

${ }^{*} p<.05,{ }^{* *} p<.01 ; \alpha=.05$ for Body Mass Index and $a=.01$ for secondary outcomes.

\section{DISCUSSION}

It was investigated whether a gamified WM training in combination with an online lifestyle intervention would lead to improved self-regulation and increased weight loss compared to a lifestyle intervention combined with sham training. On average, participants lost a small amount of weight, though the WM training did not result in additional weight loss compared to lifestyle intervention. However, the training did result in less food intake immediately after training. Results further showed a stronger increase in WM capacity in the experimental condition compared to control at posttest, and these gains were retained at one month follow-up, in line with Houben et al. (2016). In the literature there were some indications of prolonged improvements of WM following training (e.g. Holmes, Gathercole, \& Dunning, 2009; Jaeggi, Buschkuehl, Jonides, \& Shah, 2011). However, the current results stress the importance of continuous practicing, as gains in WM relative to control were lost at the six month follow-up measurement. As the effect seems to decay over time, booster WM training sessions could potentially 
elongate training effects. Hence, we encourage future studies to explore the potential added value of booster sessions. The control condition also showed an increase in performance on the WM tasks of the training. This was probably due to practice effects, though we cannot exclude the possibility that a sham training also caused (smaller) improvements of WM capacity.

The 2-back task was included as an examination of near transfer effects to a nontrained WM task. However, no significant difference between conditions after the training could be demonstrated. Unfortunately, due to technical problems, data of the 2-back task was lost for a part of the sample, and practice conditions were not equal for every measurement, resulting in that the data could not be analyzed at follow-up measurements. Previous research showed somewhat mixed results regarding reliability of the 2-back task, as correlations with other WM tasks are for instance often low (Jaeggi et al., 2010). The current study therefore provided only a limited test of near transfer effects. On self-reported executive functioning in daily life, as measured with the BRIEF-A, also no differences between conditions were found. Performance-based measures and ratings of executive function assess different underlying processes (Toplak et al., 2013): Cognitive tasks are well structured and performed under optimal circumstances, capturing the processing efficiency of cognitive abilities, whereas self-report measures assesses the extent to which someone is achieving his/her goals in daily life. The current study did not find evidence for transfer-effects to other WM measures, though due to several limitations, results should be interpreted with caution. Further research should include a more expanded test battery of WM tasks and self-report measures to study possible transfer-effects.

Interestingly, a significant difference in caloric intake during the bogus taste test after completion of the training was found. Participants in the experimental condition consumed less of the snacks relative to control. Clearly, the WM training helped these participants to act more in line with their goal to lose weight (Hofmann et al., 2008; Hofmann et al., 2012). Post-hoc analyses showed that craving moderated the effect of training on food intake. Specifically, at low levels of craving, there was no difference in caloric intake between training and control. At high levels of craving, in contrast, intake was significantly reduced in the training conditions relative to control. Hence, these findings indicate that the training is most effective in reducing intake when there is a strong impulse to consume food (i.e., craving) that requires self-regulation (cf. Hofmann et al., 2008). However, note the explorative nature of this analysis, which requires further replication. Houben et al. (2016) found that only highly restrained participants reduced their intake after WM training. In the current study, no such moderation by restraint was found but note that we included only participants who were motivated to achieve weight loss. As a limitation, it should be noted that food intake was only measured at posttest, and therefore it is unclear whether effects on food intake were retained at 
follow-up. We also did not directly measure real-world caloric intake, for instance with a food diary, as food diaries are often prone to bias and recall error. Instead, we included a laboratory-based taste test as a more reliable measure of actual food intake. However, it would be interesting for future studies to also track changes in daily food intake.

Both the experimental and control condition showed improvements over time in self-reported external and emotional eating, self-control in general, eating disorder pathology regarding shape, eating and weight, and healthy eating patterns, for up to six months after the training. In line with these results, both conditions lost a small amount of weight during the intervention period, which was retained at follow-up. The observed reduction in weight from pretest to follow-up could have been due to the lifestyle sessions provided, or simply to the fact that participants took part in an intervention for weight loss. To disentangle these effects, future research could include two extra conditions, receiving only WM training or sham training without a lifestyle intervention. Because the main focus was to examine the effectiveness of WM training, we did not specifically form or test hypotheses on the effectiveness of the lifestyle intervention. It would nevertheless be interesting for future study to explore whether participants made specific changes in their dietary intake in response to the lifestyle intervention and what types of dietary changes they were attempting to make. With respect to the main aim of the study, however, the present findings show no indication that supplementing a lifestyle intervention with WM training has any added value in reducing emotional eating, pathological thoughts related to shape, eating and weight, unhealthy eating patterns, or weight. A month is however a relatively short period of time to lose a significant amount of weight. Effects on BMI can perhaps only be expected after a prolonged period of time, as participants need time to be able to apply their increased WM capacity to weight loss strategies. In the current study, the increase in WM capacity was not maintained at six month follow-up, and therefore effects of the training on weight loss could not be anticipated due to training effects. Here as well, it would thus be interesting to examine the value of booster WM training sessions on body weight.

The current study examined the effectiveness of a general WM training. However, it would also be interesting to tailor the trainings sessions to the domain of weight loss and dieting. For instance, in inhibition training, practicing inhibition with food items (behavior-specific training) seems more effective than inhibition training in general (e.g. Allom, Mullan, et al., 2016; Houben \& Jansen, 2015; Houben et al., 2014). Perhaps the same idea applies to WM training. Some evidence suggests that food-specific WM capacity is a stronger predictor of dieting success than general WM capacity. For instance, Meule (2016) found that successful dieters did perform better than non-successful dieters on n-back task with food stimuli relative to general stimuli, and Hege et al. (2013) showed that differences in brain activity during a food-specific n-back task were related to 
successful weight loss following lifestyle treatment. Food-specific WM may be more relevant in predicting weight loss compared to general WM, for instance, because one needs to keep track of the amount of calories already consumed to be able to stick to one's diet, and because food cues have to be monitored to select when and what to eat (Boutelle \& Kirschenbaum, 1998). Further, it is possible that palatable food cues activate executive functioning in successful dieters, leading to more control over eating behavior (Meule, 2016), and it has been suggested that food-specific WM training may also reduce food cue reactivity (Jansen et al., 2015; Meule, 2016). It would therefore be interesting for future studies to explore the effectiveness of food-specific WM training on eating behavior and weight loss. From the current literature, it is also not clear what type of WM is most important in the regulation of eating behavior. A recent study provides evidence for the relationship between better visuospatial WM and healthy food intake and dietary success (Whitelock, Nouwen, Van den Akker, \& Higgs, 2018). According to these results, the current training did target the right components. Future studies should explore the role of the specific WM sub-components in the regulation of eating behavior further, as the effectivity of WM training could be further enhanced by focusing on the specific subcomponents involved in eating behavior and weight loss.

Strengths of the current study are the inclusion of an active control group and the inclusion of follow-up measurements for up to six months following training. The current study is also representative of the general population (i.e., by using a community sample). Unfortunately, the current study also had a relatively high rate of dropout during the online training (23\%), which was comparable to the study of Houben et al. (2016) (18\%), despite the serious game format of the WM training. A lack of time to complete the sessions was provided as the main reason for dropout, as sessions were considered to be very time consuming. Yet every intervention requires effort, and participants should be prepared to invest time and energy. It is not clear from prior research how many sessions of WM training are needed to be efficient. A shorter training might have led to higher adherence while still preserving the efficacy of the training. Future studies should explore the optimal amount of training sessions regarding adherence and efficiency. Interestingly, more participants of the experimental condition dropped out as compared to those randomized to control. This could be because the training took more effort to complete, as trials were more challenging. Future studies should try to keep the effort equal between conditions. Also, future studies could compare the standard, nongame WM training with our gamified training, to see whether both produce equal outcomes.

In conclusion, current results show that WM can be improved, though we found no evidence for wide transfer effects to daily life and the added value of WM training as a weight loss intervention could not be demonstrated. However, the current study did show reduced food intake immediately following WM training. Hence, it may be 
premature to conclude that WM training is not effective at all, since at least in the shortterm WM training affected food intake. We therefore encourage other researchers to replicate and extend the current findings.

\section{ACKNOWLEDGEMENTS}

This project was funded by the Maastricht University Interfaculty Program 'Eatwell'. We want to thank Berber Jacquemijns for assisting in the recruitment, screening and data collection. We are grateful to Michiel Vestjens and Richard Benning for designing and programming the gamified version of the WM-training and their excellent assistance during the execution of the study.

\section{Footnotes}

1 Participants also performed the 2-back task at FU1 and FU2. However, due to a programming error, the practice-trial was not kept constant for all participants, allowing a part of the sample to practice only once at FU1, regardless of accuracy-level at the practice-session. At FU2, all participants were only allowed to practice once. This unintended protocol violation (i.e., less practice permitted) introduced bias, and therefore data at F1 and F2 was not analyzed for the 2-back task. The mixed regression model therefore only included the change from baseline to posttest.

2 Logistic regression was performed to assess the difference between participants who completed the training and participants who dropped out during the training. The participants who dropped out did not differ from the participants who finished the training in terms of BMI, sex, education level or RS (all $p$ 's >.24). However, both age and condition ( $1=$ training versus $0=$ control) were predictive of dropout. Participants who dropped out were somewhat younger compared to the rest of the sample, $B=-.06, p=.04$, and more often in the experimental condition, $B=1.50, p=.02$. All randomized participants were included in the analysis, even if they were only measured at baseline, and age, sex and education level were included as covariates in all analyses, using maximum likelihood estimation. This method of treatment effect estimation is valid without imputation of missing outcome values even if dropout is related to treatment or covariates or the baseline of the outcome at hand (Verbeke \& Molenberghs, 2000).

3 Due to a technical error in the saving of responses, data of the 2-back task was lost at random for some participants, resulting in a sample of $n=74$ at baseline and $n=64$ at posttest.

4 Note that beginning and end time per practiced task at home was recorded automatically, though from these data cannot be derived whether participants spent all the time actively on the training, or took some breaks in between. The difference between conditions with respect to the mean time per session was not statistically significant, $t(1,67)=1.83, p>.05$. One participant was excluded from this particular analysis, because the mean time per session for this person was a clear outlier ( $M=510.90$ minutes).

5 Regarding the 2-back data, there was one outlier identified on the bottom end of the data at baseline and three outliers were identified on the bottom end of the data at posttest. These scores were replaced by the nearest value which was not an outlier minus one point for the outlier identified at baseline, and minus one, two and three points for the outliers identified at posttest. The analyses without adjustment for outliers also showed no condition*time interaction $(p>.085)$. 


\section{SUPPLEMENTARY MATERIALS}

S1 Short description of the content of the online lifestyle intervention, displayed per session.

\section{Session 1}

- Dieting and healthy weight loss

- Energy balance

- Body Mass Index

- Calculating personal energy need with Harris-Benedict formula

- Keep track of daily intake with online tool

- Creating personal motivation card with reasons to lose weight

- Homework: Look at motivation card at least two times a day and keep track of daily caloric intake

\section{Session 2}

- Nutrients

- Heathy weight loss

- Obesogenic environment

- Personal diet plan

- Fact or myth quiz about nutrition

- Homework: Apply personal diet plan and continue using the motivation card at least twice a day

\section{Session 3}

- Usefulness of exercising

- Daily exercise recommended by Nederlandse Norm Gezond Bewegen

- Metabolic Equivalent of Task

- Keep track of daily exercise

- Spontaneous and planned exercise

- Personal exercise plan

- Sleep quality

- Tips for healthy sleep hygiene

- Homework: Keep track of daily exercise, apply personal exercise plan and apply tips for sleep hygiene

\section{Session 4}

- Difficult moments (e.g. holidays, parties) and how to handle them

- Make a plan for a difficult moment

- Apply plan in daily life and evaluate

- When is a diet finished?

- Tips to retain a healthy body weight

- Evaluation of sessions

S2 An overview of all tasks and questionnaires provided at the different time points of the study, in order of administration.

\begin{tabular}{|c|c|c|c|c|}
\hline Measures & Baseline & Posttest & Follow-up 1 & Follow-up 2 \\
\hline Food intake (bogus taste test) & & $x$ & & \\
\hline WM span - assessment-version trained tasks & $x$ & $x$ & $x$ & $x$ \\
\hline 2-back task & $x$ & $x$ & & \\
\hline $\begin{array}{l}\text { Behavioral Rating Inventory of Executive } \\
\text { Functioning - Adult version }\end{array}$ & $x$ & $x$ & $x$ & $x$ \\
\hline Healthy Eating Questionnaire & $x$ & $x$ & $x$ & $x$ \\
\hline Dutch Eating Behavior Questionnaire & $x$ & $x$ & $x$ & $x$ \\
\hline Eating Disorder Examination -Questionnaire & $x$ & $x$ & $x$ & $x$ \\
\hline Restraint Scale & $x$ & & & \\
\hline Brief Self-Control Scale & $\mathrm{X}$ & $x$ & $x$ & $x$ \\
\hline Demographics & $x$ & & & \\
\hline Height & $x$ & & & \\
\hline Weight & $x$ & $\mathrm{X}$ & $x$ & $x$ \\
\hline
\end{tabular}


S3 Participant characteristics at baseline. Means and standard deviations per condition are displayed.

\begin{tabular}{lccc} 
& Training $(\boldsymbol{n}=\mathbf{5 1})$ & Control $(\boldsymbol{n}=\mathbf{4 0})$ & Range \\
\hline Age & $46.29(11.89)$ & $50.10(8.60)$ & $22-61$ \\
Body Mass Index $\left(\mathrm{kg} / \mathrm{m}^{2}\right)$ & $30.96(3.64)$ & $30.49(3.97)$ & $25.05-41.94$ \\
Sex $(\%$ female) & $76.5 \%$ & $72.5 \%$ & - \\
Restraint scale (RS) & $16.10(4.58)$ & $15.13(4.42)$ & $5-29$ \\
DEBQ - dietary restraint & $3.18(0.58$ & $3.01(0.59)$ & $1.7-4.6$ \\
Working Memory (WM) span & $5.15(1.02)$ & $5.12(0.83)$ & $3.33-7.67$
\end{tabular}

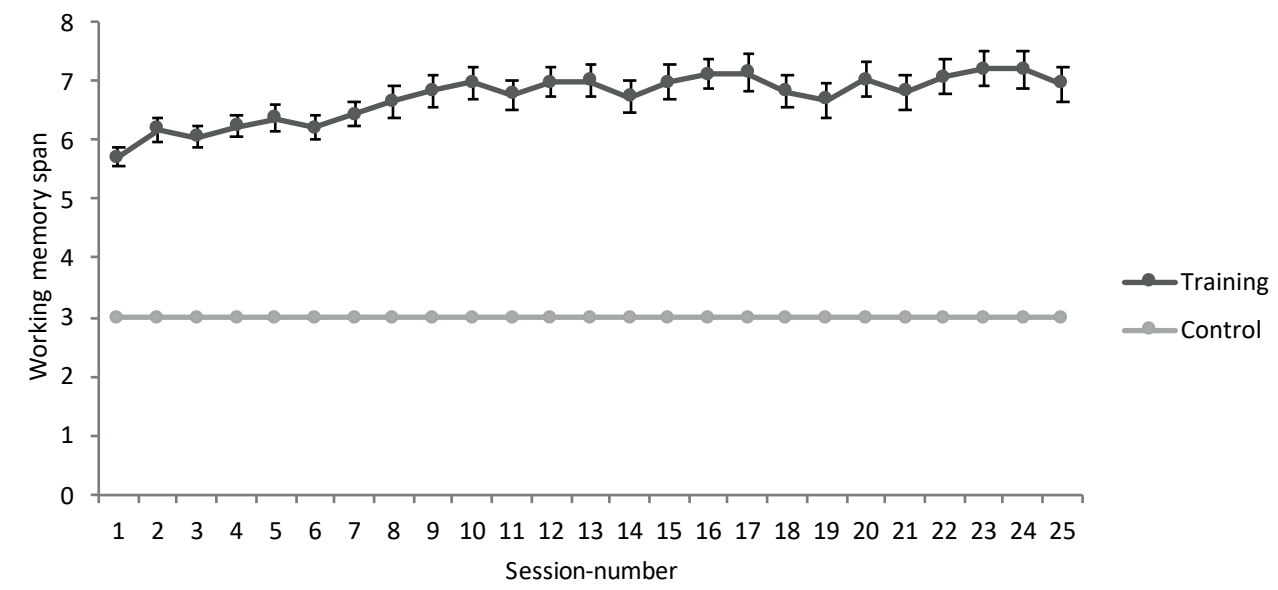

S4 The number of items (WM span) that could be correctly recalled at the end of each training session. The average of the three practiced tasks is displayed, separately for the experimental and control condition. Error bars represent the standard error of the mean. 


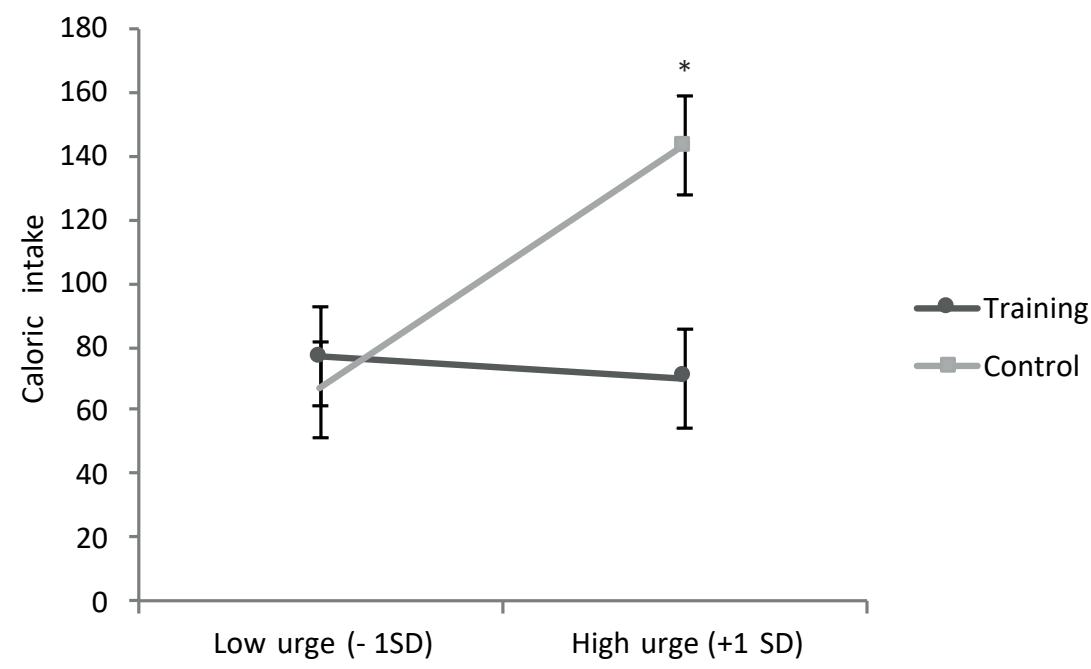

S5 Estimated marginal means for caloric intake at posttest. Means are displayed separately for participants with a low urge to eat (low craving) and a high urge to eat (high craving). Error bars represent the standard error of the mean.

Note. ${ }^{*}$ indicates significant differences at $p<.01$.

S6 Effect of training on Body Mass Index at different points in time, established with mixed linear regression correcting for age, sex and education level $(N=91)$.

\begin{tabular}{lcccccc} 
& Estimate & S.E. & $\boldsymbol{t}$ & $\boldsymbol{p}$ & \multicolumn{2}{c}{$\mathbf{9 5 \%} \mathbf{C l}$} \\
\hline Intercept & 30.70 & 2.48 & 12.36 & - & 25.77 & 35.64 \\
Condition (0=control, 1=experimental) & 0.43 & 0.84 & 0.51 & .61 & -1.24 & 2.09 \\
Posttest & -0.40 & 0.13 & -3.15 & .002 & -0.66 & -0.15 \\
FU1 & -0.38 & 0.17 & -2.27 & .03 & -0.72 & -0.05 \\
FU2 & -0.36 & 0.26 & -1.41 & .16 & -0.87 & 0.15 \\
Condition x Posttest & 0.08 & 0.18 & 0.43 & .67 & -0.29 & 0.45 \\
Condition x FU1 & -0.09 & 0.24 & -0.39 & .70 & -0.58 & 0.39 \\
Condition x FU2 & -0.24 & 0.36 & -0.66 & .51 & -0.96 & 0.49 \\
Age & -0.00 & 0.04 & -0.83 & .93 & -0.08 & 0.07 \\
Sex (0=male, 1=female) & 0.01 & 0.95 & 0.01 & .99 & -1.88 & 1.91 \\
Education1 (secondary education) & -0.20 & 1.52 & -0.13 & .90 & -3.23 & 2.83 \\
Education2 (higher education) & 0.07 & 1.47 & 0.05 & .96 & -2.86 & 3.00
\end{tabular}

Note. Estimate $>0$ indicates a higher score in the experimental group. 
A

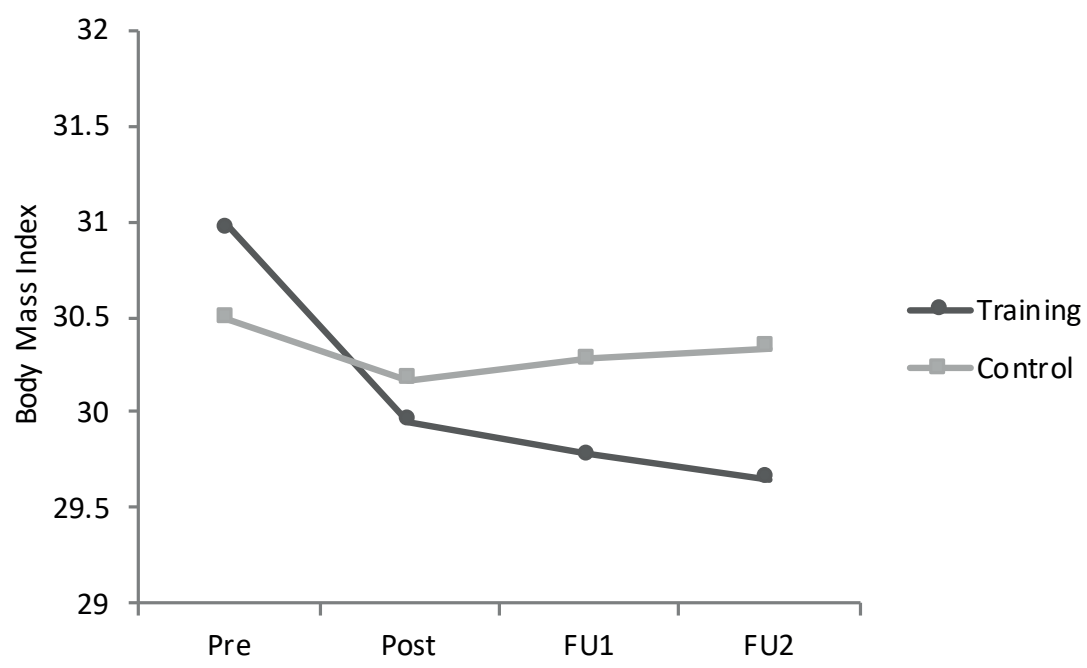

B

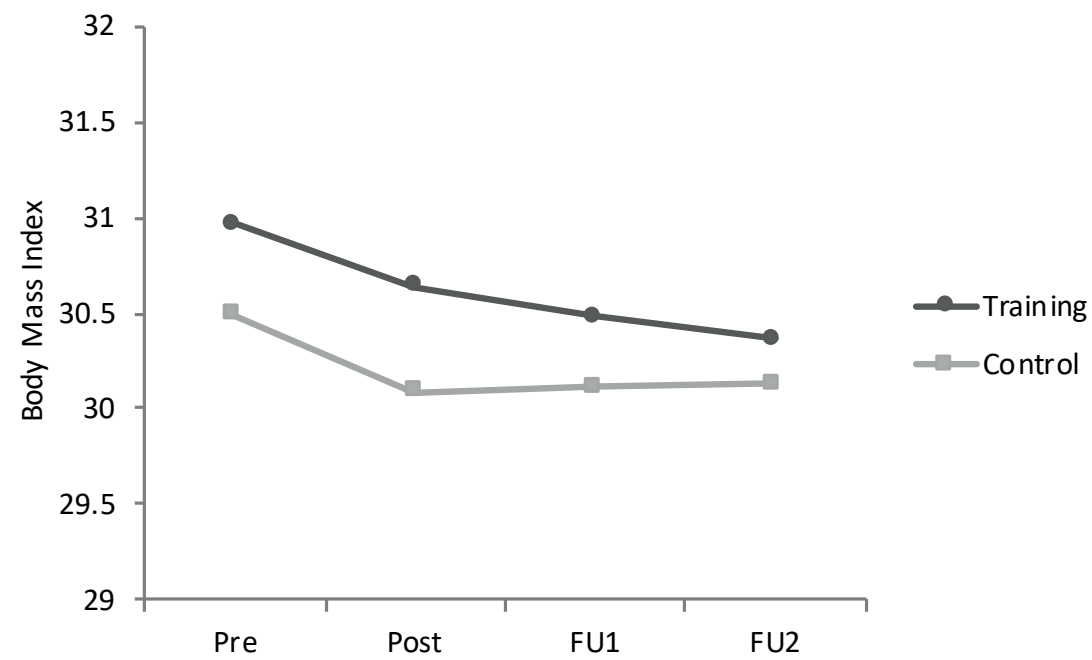

S7 Observed means (A) and estimated means (B) based on the final mixed model (REML estimation) for Body Mass Index. For the observed means, $N$ varies per measurement due to dropout or missing data, which may bias observed means. 
S8. Effect of training on percentage BMI-loss at posttest, follow up1 and follow-up 2, established with mixed linear regression correcting for age, sex and education level $(N=70)$ a.

\begin{tabular}{lcccccc} 
& Estimate & S.E. & $\boldsymbol{t}$ & $\boldsymbol{p}$ & \multicolumn{2}{c}{$95 \% \mathbf{C I}$} \\
\hline Intercept & -2.84 & 1.82 & -1.56 & - & -6.49 & 0.80 \\
Condition (0=control, 1=experimental) & 0.21 & 0.62 & 0.33 & .74 & -1.44 & 1.03 \\
FU1 & -0.03 & 0.25 & 2.01 & .05 & -0.54 & 0.47 \\
FU2 & -0.11 & 0.63 & 1.26 & .21 & -1.36 & 1.14 \\
Condition x FU1 & 0.59 & 0.36 & 1.64 & .11 & -0.13 & 1.32 \\
Condition x FU2 & 0.93 & 0.88 & 1.06 & .29 & -0.83 & 2.69 \\
Age & 0.07 & 0.03 & 2.27 & .03 & 0.01 & 0.13 \\
Sex (0=male, 1=female) & -0.53 & 0.67 & -0.79 & .43 & -1.87 & 0.81 \\
Education1 (secondary education) & 1.06 & 1.11 & 0.95 & .35 & -1.16 & 3.28 \\
Education2 (higher education) & 1.39 & 1.05 & 1.32 & .19 & -0.71 & 3.50
\end{tabular}

a There was one outlier identified on the top end of the data at FU2 (percentage BMI-loss of 21.16\%). This percentage was replaced by the nearest value which was not an outlier plus one percent (e.g. 14.56\%). The analyses without adjustment for this outlier also showed no condition*time interaction effect $(p=.25)$.

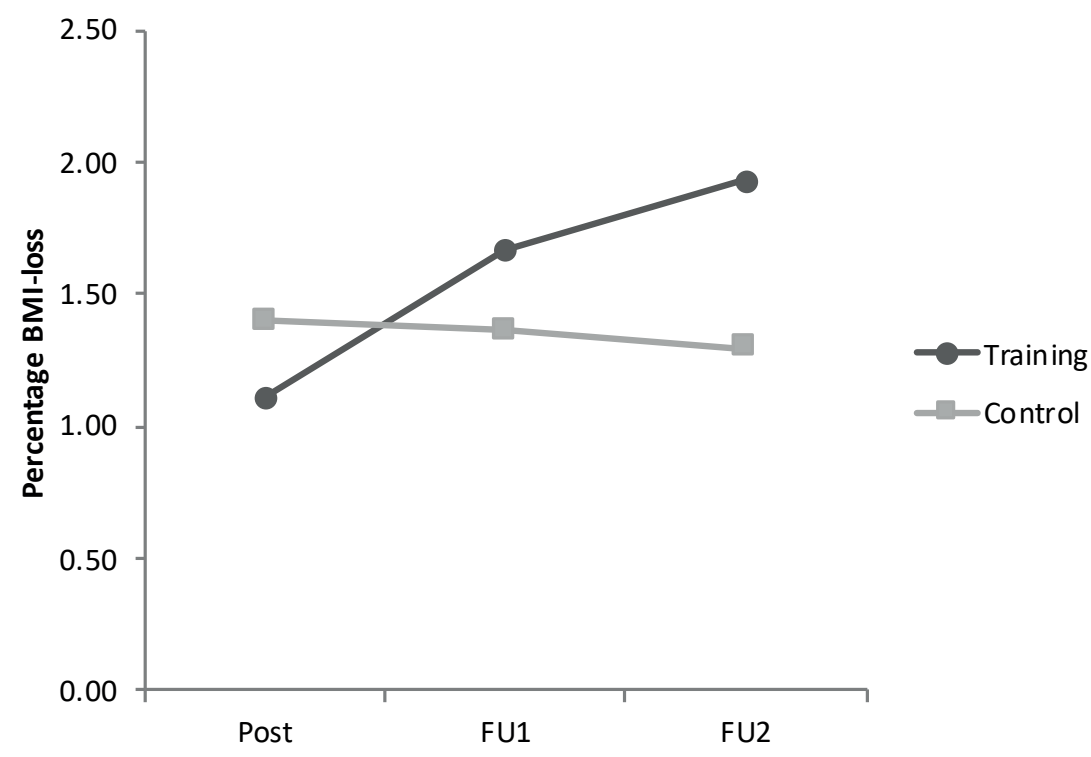

S9. Estimated means of at posttest, follow up1 and follow-up 2, based on the final mixed model correcting for age, sex and education level (REML estimation) for percentage BMI-loss $(N=70)^{\text {a }}$. Participants who dropped-out during the training $(n=21)$ are not included in this analyses, which may bias the results.

a There was one outlier identified on the top end of the data at FU2 (percentage BMI-loss of 21.16\%). This percentage was replaced by the nearest value which was not an outlier plus one percent (e.g. 14.56\%). 


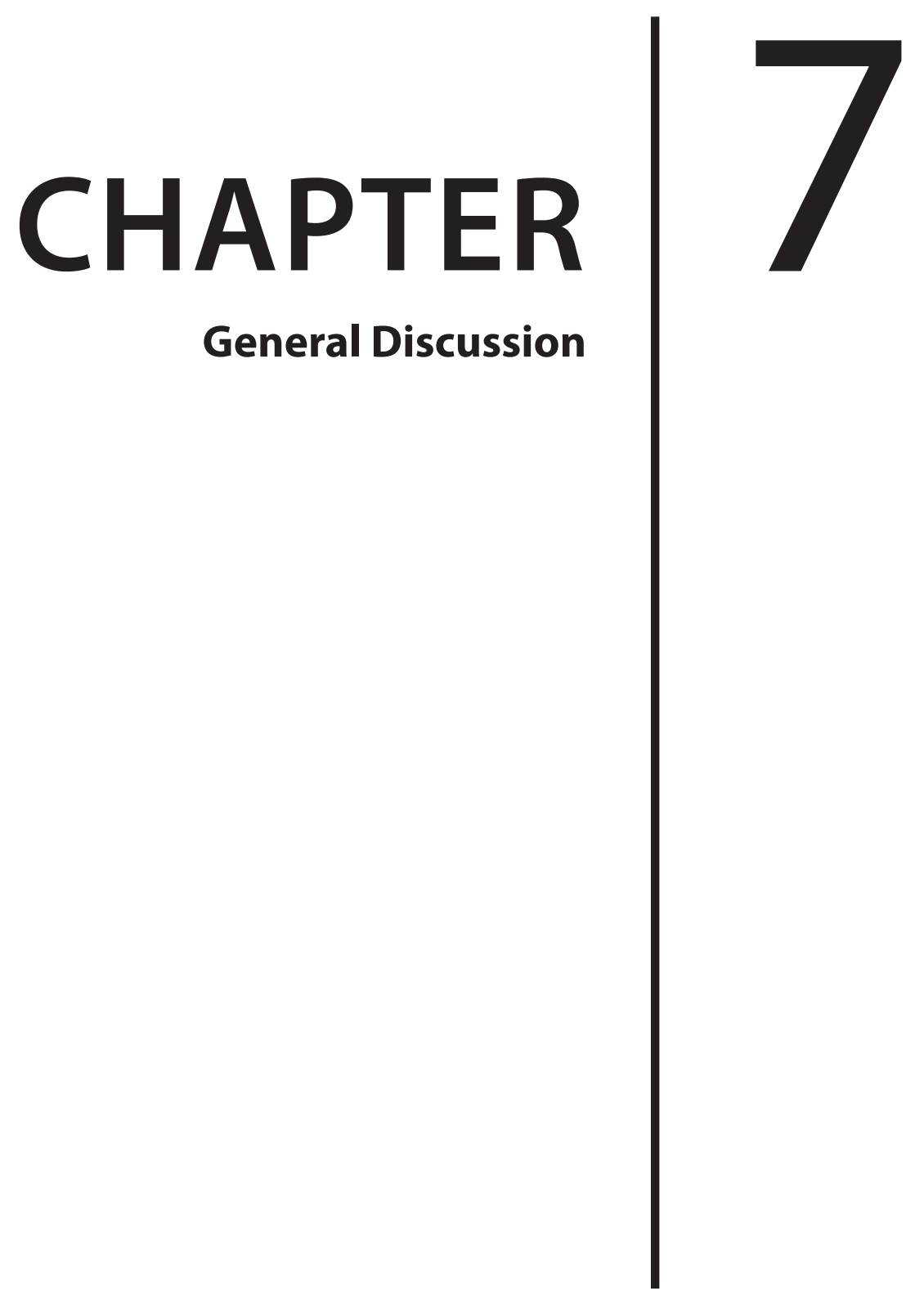



The current dissertation addressed the role and trainability of cognitive control in eating behavior and weight loss. The topics covered in this dissertation can be divided into two themes. In part 1, we focused on the cognitive profile of obesity, with a specific focus on the role of the core facets of executive function (EF): inhibition, working memory and shifting, and the role of the EF-related construct of time orientation including delay discounting and Consideration of Future Consequences (CFC). In part 2, we focused on manipulations of cognitive control, with a specific focus on manipulations addressing delay discounting, shifting and working memory. A summary and more indepth discussion of the results of the research findings per subpart is provided, and attention is given to methodical considerations. The chapter ends with a conclusion on the current state of research in this area and future directions.

\section{PART 1.}

\section{The Cognitive Profile of Obesity: Establishing the Association of Executive Functioning and Time Orientation with Eating Behavior and Weight Loss}

The studies described in chapter $\mathbf{2}$ and chapter $\mathbf{3}$ both aimed to investigate the hypothesized role of the main facets of EF and time orientation in eating behavior, obesity and weight loss. In chapter $\mathbf{2}$, obese patients of a weight loss clinic were compared with healthy weight controls on self-report and behavioral measures of inhibition, working memory and shifting, and a questionnaire addressing delay discounting. We studied both general inhibition and inhibition for food-stimuli, as previous research suggested that obesity is associated with weaker behavior-specific inhibition instead of general inhibition (e.g. Houben et al., 2014; Nederkoorn et al., 2012). A second aim of this study was to assess whether EF and delay discounting would be predictive of weight loss after six months of multidisciplinary treatment. In chapter 3, we studied the EFrelated construct of time orientation in a general community sample. Specifically, we focused on general and food-specific measures of the extent to which individuals took consideration of future consequences (CFC) in their decision making and how quickly a reward devaluated in time (delay discounting). In the next subsections, results of chapter $\mathbf{2}$ and chapter $\mathbf{3}$ will be summarized and discussed for each main facet of EF and time orientation separately.

\section{Inhibition}

A confrontation with food cues (e.g. food stimuli) possibly heightens temptation for an individual trying to restrain intake, thereby triggering an impulsive reaction (Hofmann \& Van Dillen, 2012). The individuals with obesity displayed both weaker general and foodspecific inhibition on the Stop-Signal Task (SST) compared to healthy weight controls, 
thus a more pronounced inhibitory deficit in response to food stimuli could not be established. Previous research which addressed this question included overweight individuals in their 'obese' group (Houben et al., 2014; Nederkoorn et al., 2012). It may be that differences in general inhibition performance are only apparent at the higher end of the weight spectrum, and examining EF in a less severe sample of overweight instead of obese individuals might make it more difficult to detect significant differences in comparison to healthy weight controls. A recently published meta-analysis including 39 studies on differences in inhibition between obese, overweight and healthy weight individuals found that both overweight and obese individuals showed weaker inhibition relative to the healthy weight controls (Yang et al., 2018). The authors made no distinction between general and food-specific inhibition in their results. In conclusion, current results are in line with the literature and postulate that weaker inhibition, whether it is general or food-specific, is associated with obesity.

Interestingly, whereas both the SST (general and food-specific) and self-reported inhibition were associated with obesity, only stronger self-reported inhibition in daily life was predictive of a greater weight change. It is assumed that a self-report scale about inhibition measures behaviors that are strongly associated with processes assessed by behavioral inhibition tasks. In chapter 2, correlations between both behavioral and self-report measures for inhibition, working memory and shifting were weak, in line with results of a meta-analysis (Duckworth \& Kern, 2011). Even though there is a conceptual overlap, both types of measurements seem to measure a different element of self-control (Allom, Panetta, et al., 2016); self-reported inhibition seems to assess a self-control capacity that is directly related to weight loss success, while the SST seems to asses a distinct self-control process. Thus, whereas weight loss success does not seem to be associated with less efficient inhibition in structured situations as captured in the performance on the SST, it does seem to be associated with less successful goal pursuit as indicated by self-reported difficulties with inhibition in daily life (Toplak et al., 2013).

\section{Working memory}

We did not find a difference between individuals with obesity and healthy weight controls on performance on the 2-back task, whereas the obese group did report weaker working memory in daily life. As discussed in the previous paragraph, selfreport measures and behavioral tasks seem to capture different aspects of EF and should thus not be used interchangeably (Allom, Panetta, et al., 2016; Toplak et al., 2013). Interestingly, the 2-back task did show a correlation with all other EF-measures, suggesting that this task captures some latent EF-factor which is shared by all measures. In line with the previously identified important role of working memory in successful self-regulation (Dohle et al., 2018; Hofmann et al., 2008; Hofmann et al., 2012) and 
consistent with previous research (Meule, 2016; Whitelock, Nouwen, van den Akker, \& Higgs, 2018), we found better working memory performance to be the most important predictor of weight loss.

Studying specific associations between EF and food intake and mapping specific weight loss-related behaviors onto specific executive skills is an important next step for future research, as it is difficult to conceptualize how outcome variables provided by behavioral tasks (i.e., accuracy or reaction time) exactly translate into daily behaviors (Gettens \& Gorin, 2017). Recently, research started to explore more specific associations of working memory processes and eating behavior. For instance, Meule (2016) found that successful dieters did not show better working memory performance in general relative to unsuccessful dieters, but specifically in response to food cues (e.g. on a foodspecific $n$-back task), similar to the suggested food-specificity of inhibition weaknesses in obesity (Houben et al., 2014; Nederkoorn et al., 2012). Whitelock et al. (2018) showed that especially better visuospatial working memory was related to both healthy food intake and dietary success. They suggest that a better visuospatial working memory could allow dieters to deal more efficiently with food cravings, which are mainly visuospatial in nature and thus consume visuospatial working memory resources (Kemps \& Tiggemann, 2010; Tiggemann \& Kemps, 2005). Future research should continue to investigate the specific underlying processes and overt behaviors that explain how working memory influences weight loss success.

\section{Shifting}

In chapter 2, no significant difference in self-reported shifting between obese individuals and healthy weight controls was reported, though results were in the expected direction, with less efficient shifting in daily life being reported by the individuals with obesity. We could not replicate results of previous research indicating an association between obesity and weaker shifting as measured with the Trail Making Test (TMT) (Cserjési, Molnár, Luminet, \& Lénárd, 2007; Gunstad et al., 2007; Verdejo-García et al., 2010), though other studies also failed to find this association (Ariza et al., 2012; Boeka \& Lokken, 2008). In a recent meta-analysis of 24 studies assessing BMI and shifting the authors concluded that obesity shows a weak to moderate association with shifting deficits, and this effect does not seem to be moderated by the type of task used (Yang et al., 2018). Thus, obesity appears to be weakly associated with weaker shifting, though some studies including ours failed to demonstrate this difference on the TMT.

Results of chapter $\mathbf{2}$ revealed that self-reported shifting was negatively associated with weight loss, indicating that less efficient cognitive flexibility was associated with a greater BMI decrease. Shifting as measured with the TMT was not related to weight loss. Old reactions are automatically activated in new or difficult situations, in which we tend 
to rely on habitual responses. During a weight loss intervention, effective switching is required to overcome old habits and initiate an alternative and goal-congruent behavioral response (Allan, Johnston, \& Campbell, 2010). Earlier research provides some indications that someone who displays flexible dietary restraint is more successful in weight loss maintenance than someone displaying rigid restraint (Hofmann et al., 2012; Teixeira et al., 2015), thus flexible control seems a more adaptive and functional dieting strategy with more favorable outcomes than rigid control (Meule, 2017). However, effective switching should occur at the level of means to reach a goal (i.e., switching to a different strategy to adapt to new circumstances or overcome an obstacle), though not at the level of goals (i.e., switching to hedonic goals) (Dohle et al., 2018; Hofmann et al., 2012). Thus, there seems to be a fine line between adaptive shifting at the level of means and non-desirable switching at the level of goals. The unexpected finding that obese individuals who scored worse on shifting lost more weight may reflect that these individuals held on more rigidly to their health goals, which could have a beneficial effect on short-term weight loss.

\section{Time orientation}

Delay discounting was neither related to BMI nor weight loss in the results described in chapter 2, thus we found no evidence for the predictive value of delay discounting as measured with the Monetary Choice Questionnaire (MCQ). In chapter 3, we assessed time orientation in general (i.e., delay discounting with money as a reinforcer and general CFC) and food-specific time orientation, with CFC-food (i.e., CFC regarding food intake and health) and MCQ-snack (i.e., delay discounting with snack food as a reinforcer). Results revealed CFC-food to be the single best predictor of self-reported eating behavior, with both more consideration of immediate consequences and less consideration of future consequences regarding food choice and health being associated with unhealthier eating behavior in daily life. In contrast to results of chapter $\mathbf{2}$, in which individuals with obesity were compared with healthy weight controls, a positive though small correlation between discounting for money and BMI was discovered in chapter 3. Originally, it was assumed that time orientation is a domain-general construct. Research in the field of delay discounting provides some evidence that individual discount rates differ when different reinforcers are used. For instance, people's discounting of delayed money and health outcomes were uncorrelated as were discounting of real, consumable rewards and hypothetical money (Green \& Myerson, 2013), and obese individuals discounted food more steeply than healthy weight controls, but not money or vouchers (Schiff et al., 2016). Also, food-specific discounting predicted weight loss success after a twelve-week low calorie diet (Weygandt et al., 2013). Thus, how an individual discounts one outcome does not necessarily indicate how that individual will discount all types of 
outcomes (Weatherly \& Ferraro, 2011; Weatherly \& Terrell, 2010), and delay discounting is probably measured best with behavior-specific measures.

Non-significant results of chapter 2 can possibly be explained by the use of monetary rewards to predict behavior in a different domain (i.e., health behavior). In chapter 3, we therefore developed a behavior-specific measure of delay discounting by adapting the original MCQ (Kirby et al., 1999). We replaced monetary values with snack foods, whereby participants could choose their favorite snack from four different sweet and savory snacks. Results revealed that monetary rewards were discounted more steely than consumable rewards, in line with earlier research (Estle, Green, Myerson, \& Holt, 2007; Odum \& Rainaud, 2003). Our behavior-specific measure was neither significantly related to eating behavior nor BMI, suggesting that this construct does not predict eating behavior or BMI. However, concerning delay discounting, the 'dilemma' for a dieter would entail choosing between a palatable snack now (i.e., the small, immediate reward) and future weight loss and health benefits (i.e., the bigger, delayed reward). By providing a choice between a small amount of snacks now or a bigger amount of snacks later, our food-specific measure did not fully capture this dilemma, as someone displaying less discounting of the future in this discounting task would receive a future reward consisting of even more of the (unhealthy) food. It would be interesting for future research to develop a delay discounting task mirroring this weight loss dilemma more closely, for instance the choice between an immediately available snack and weight loss which would be available at different times in the future (Epstein et al., 2010)

An important finding of the current research is the behavior-specificity of the construct of time orientation, as results of chapter 3 clearly indicated CFC-food to be the single best predictor of eating behavior, in line with earlier research (Hall et al., 2012; van Beek et al., 2013). Neither delay discounting nor general CFC seems to be related to self-reported eating behavior. Thus, time orientation varies within individuals, between domains (e.g. health and money) and even within domains (e.g. exercising and eating, which both belong to the domain of health; Hall et al., 2012; van Beek et al., 2013). Hall et al. (2012) documented that the facilitating effect of future-oriented thinking was mediated by intention-strength, thus a future time perspective lead to more weight management behaviors via a stronger intention to perform them. Given the association of CFC-food with eating behavior, these results indicate that behavior-specific CFC could be an interesting target for intervention, which will be further discussed in the second part of this discussion.

\section{Some methodological considerations Measuring EF, a considerable challenge}

The two studies as described in chapter $\mathbf{2}$ and $\mathbf{3}$ provide mixed results for the role of 
EF and time orientation in obesity, weight loss and eating behavior and our hypotheses were only partly confirmed. Results are not fully in line with the current literature (e.g. Yang et al., 2018). One possible explanation for these mixed findings can be found in the diversity of tasks developed and used to measure EF and EF-related constructs. Even comparison with studies administrating the exact same tasks is difficult, because validated norm scores are often lacking, and different studies use different versions and outcome measures of the same computer tasks (Halberstadt et al., 2013). Also, Schmiedek, Lövdén, and Lindenberger (2014) strikingly state that no task or paradigm will ever be process-pure, as there will always be other task-specific processes involved in the performance of the participant, besides the key process of interest. Another possible explanation can be found in the sensitivity of the tasks used. Originally, tasks to measure EF were developed to identify prefrontal brain damage in patients (e.g. Fitzpatrick et al., 2013). Thus, these tasks mainly capture performance at either a pathological or impairment level (Whyte et al., 1996). However, in obesity, differences in EF may be quite subtle (Fitzpatrick et al., 2013). Small to medium effect sizes of weaknesses in inhibition, working memory and shifting in obese individuals have been reported recently (hedges' $g-.36,-.33$ and -.37 respectively; Yang et al., 2018). It seems that obese individuals do show subtle impairments in EF and decision making, though current and other studies failed to detect this because they vary in methodology and sample selection, and they were possibly underpowered to detect such small effects.

\section{Initial weight loss versus weight loss maintenance}

As a limitation of the prospective study described in chapter $\mathbf{2}$, it should be noted that we only followed participants up to six months of multidisciplinary weight loss treatment. However, it is not initial weight loss following treatment that is the biggest problem, but rather weight loss maintenance (Ikeda et al., 2005; Yanovski \& Yanovski, 2003), and current results provide no indications about future maintenance of the weight loss achieved. It has been suggested that self-regulation becomes even more important when treatment ends, as with the end of the treatment also the external regulation coming from the treatment program ends (Halberstadt et al., 2013). At this point, maintaining the new healthy lifestyle has to be internally regulated, requiring strong EF. For a more complete understanding of the role of EF and delay discounting in long-term weight regulation, future research should distinguish between their role in weight gain, weight loss and weight loss maintenance (Gettens \& Gorin, 2017).

\section{The issue of causality}

Current cross-sectional and prospective designs do not shed light on the causal relationship of BMI and EF: does weaker EF cause obesity or does obesity lead to weaker 
$\mathrm{EF}$ ? There is evidence for obesity being directly related to impaired $\mathrm{EF}$, due to underlying biological factors such as reduced blood flow to the areas of the brain that control $E F$, increased adipocytes and abnormalities in glucose and insulin regulation (Boeka \& Lokken, 2008; Smith et al., 2011). If weaker EF is mainly a consequence of obesity, effects could already be reversed to some extent by weight loss only. Some evidence for improvement of EF following weight loss exists in bariatric surgery populations, where indications have been found that EF improved post-surgery (Alosco et al., 2014; Spitznagel, Alosco, et al., 2013; Spitznagel, Garcia, et al., 2013), and the results of a recently published meta-analysis endorse the significant positive effect of weight loss on EF (Veronese et al., 2017). There is also evidence for weaker EF leading to obesity via for instance heightened unhealthy food intake (e.g. Allan et al., 2010; Guerrieri, Nederkoorn, Schrooten, Martijn, \& Jansen, 2009; Hall, 2012; Limbers \& Young, 2015), rendering individuals with weak EF more predisposed to become obese (Dohle et al., 2018; Hofmann et al., 2012). A genetic vulnerability could underlie this weaker EF, increasing the risk for obesity. The existence of a third variable that impacts both the development of obesity and executive dysfunction is however also a possibility.

To conclude, at the moment, it is not yet clear which framework describes best how EF, obesity and obesity-related behaviors (e.g. overeating, disinhibited eating, less physical activity) are related with each other (Liang, Matheson, Kaye, \& Boutelle, 2014). As there is evidence for both weaker EF 'causing' obesity and obesity 'affecting' EF, a bidirectional association entailing a 'vicious circle' between EF and obesity seems most likely (Kanoski \& Davidson, 2011; Sellbom \& Gunstad, 2012). Either way, to maintain a healthy lifestyle and achieve effective weight loss, this vicious cycle should be broken. Experimental study-designs could shed more light on these associations. This was the focus of the second part of this dissertation.

\section{PART 2.}

\section{Manipulating Cognitive Control: Episodic Future Thinking, Shifting and Working Memory Training}

\section{The potential of episodic future thinking to alter time orientation}

During the past years, a body of research on episodic future thinking has emerged, expanding our knowledge on episodic future thinking and underlying working mechanisms. Whereas training of EF seems related to a top-down approach, strengthening the boundary conditions underlying the reflective system, episodic future thinking seems to allow someone to switch the focus from 'hot' or impulsive to 'cool' or reflective cognition in a tempting situation. The fronto-parietal network projects future benefits (by prospective imagery), favouring top-down control for long term 
benefits (McClure et al., 2004). In daily life, people tend to think about a future event frequently, and these thoughts tend to be positively biased and related to goal pursuit and emotion regulation (Barsics, Van der Linden, \& D'Argembeau, 2016). In chapter 4, we studied a manipulation of episodic future thinking and food-specific episodic future thinking. Some first evidence emerged that episodic future thinking could reduce delay discounting and possibly be of use as a manipulation to reduce food intake (Daniel et al., 2013b; Peters \& Büchel, 2010). The study described in chapter 4 built on this preliminary evidence. We showed that engagement in episodic future thinking indeed reduced delay discounting for money, in line with earlier results (Benoit et al., 2011; Peters \& Büchel, 2010; Sheffer et al., 2016). Lin and Epstein (2014) found that episodic future thinking reduced delay discounting most in individuals with high working memory capacity, and speculate that individuals who have weaker working memory may be less capable of delaying gratification because they have difficulties to vividly visualize future outcomes. This suggests that working memory training could possibly enhance the effect of episodic future thinking on delay discounting, an interesting possibility that has not been studied yet. These results indicate that delay discounting and working memory are closely related constructs, which is in line with results of Bickel et al. (2011), who showed that a working memory training lead to a reduction in delay discounting, and Wesley and Bickel (2014) who identified a shared brain location for both working memory and delay discounting processes.

With respect to food intake, episodic future thinking reduced food intake in a laboratory setting in adults (Daniel et al., 2013b) and children (Daniel et al., 2015) in both obese and lean women (Daniel et al., 2013a). A study testing the effectiveness of episodic future thinking in overweight and obese families in addition to a behavioral weight loss intervention provided preliminary promising results (Sze, Daniel, Kilanowski, Collins, \& Epstein, 2015). Children and parents in the episodic future thinking group did report a marginally significant lower food intake, and parents in the episodic future thinking group lost more weight than control. In chapter $\mathbf{4}$, we found that food-specific episodic future thinking reduced food intake relative to control, whereas for general episodic future thinking and episodic past thinking, no differences in food intake could be discovered between conditions, in line with our expectations. Delay discounting and food intake were however not associated with each other, thus results for food intake were not mediated by delay discounting, questioning the ability of a general measure of delay discounting (i.e., discount rate for money) to capture the underlying mechanism causing this reduction in food intake. These results however do indicate that foodspecific episodic future thinking is a more suitable intervention than general episodic future thinking for targeting eating behavior. In line with these results, O'Donnell, Daniel, and Epstein (2017) found that financial-oriented episodic future thinking was more 
effective than general episodic future thinking in reducing monetary delay discounting. In addition, eating-goal-related episodic future thinking reduced food intake in obese and overweight women (O'Neill, Daniel, \& Epstein, 2016). Thus, effects of episodic future thinking are stronger when the future event is related to topic of the choice that has to be made, which thus suggests that effects are not merely due to the inducement of a general future orientation, but entail a behavior-specific effect (Schacter, Benoit, \& Szpunar, 2017).

As displayed by results of chapter 4, the manipulation of episodic future thinking was effective while being applied during an eating opportunity (i.e., participants composed future images while they were given the opportunity to snack). In a tempting situation, activation of the executive system is needed to regulate behavior in line with longterm goals. Thus, as this specific moment, someone should engage in episodic future thinking to counteract an impulsive choice. Delay discounting has been considered to be a trait variable (Odum, 2011), though other research showed that delay discounting varies according to the context or factors like metabolic state (Manasse et al., 2015) and concerns over weight control (Budría, Lacomba, Lagos, \& Swedberg, 2012). Also, delay discounting seems open to change, for instance by engaging in episodic future thinking or working memory training (for a review see Koffarnus et al., 2013). Thus, the question remains whether delay discounting can be considered a trait or state variable, and likewise whether episodic future thinking influences behavior via state or trait effects. This question is important when considering the potential long-term effectiveness of interventions based on episodic future thinking (McClelland et al., 2016). Previous studies only report short-term changes in delay discounting, immediately following the training or manipulation, and episodic future thinking has been categorized as a framing effect (Koffarnus et al., 2013). However, to influence eating behavior substantially, effects should be long-lasting. Future research should focus on longitudinal research and long-term stability of changes in delay discounting to understand the state/trait related nature of delay discounting in obesity (McClelland et al., 2016). An interesting possibility for future intervention would be to instruct individuals to engage in episodic future thinking at random moments during the day, favoring future-oriented decision making thorough the day.

\section{Training executive functioning: Working memory training}

Houben et al. (2016) were the first to study the effects of working memory training on eating behavior and weight loss. Participants completed either 25 sessions of working memory training or sham training. Afterwards, participants worried less about food, weight and their body, and they reported to eat less often following negative emotions (Houben et al., 2016). Even though no direct effects on food intake were 
found, an interaction between restrained eating and condition was discovered: when inspecting results among the highly restrained eaters, the working memory training group consumed less snacks than the control group, and these results were retained at follow-up after one month. Thus, the working memory training seemed to help these participants to better pursue their goal to restrict intake. Importantly, no weight loss was discovered among participants at posttest and follow-up.

The working memory training study described in chapter 6 was a direct followup on the study by Houben et al (2016), with some important changes in the design: Participants were screened to have sufficient motivation to actually work for weight loss, and the working memory training was offered in combination with online lifestyle tips, to provide every participant with sufficient knowledge about a healthy lifestyle to achieve weight loss. Results revealed that the EF training improved working memory, as displayed by a better performance of the experimental group relative to control on the trained working memory tasks. However, these improvements could not be retained at the six-month follow-up. Improvements in untrained tasks relying on the same EF are typically classified as 'near transfer', whereas improvements in tasks or behaviors relying on different cognitive abilities which however are correlated with the specific EF are considered as a 'far transfer' effect. Near transfer effects are considered a requirement for far transfer effects (e.g. Harrison et al., 2013). However, how much improvement can be reached and how long these improvements last is not clear yet. Based on the current literature, it seems that individuals only improve on what they practiced; improvement does not seem to transfer to other non-trained skills (Diamond \& Ling, 2016; MelbyLervåg \& Hulme, 2013; von Bastian \& Oberauer, 2013). In line with this, no differences between the experimental and control condition could be discovered on any of the secondary outcome measures reported in chapter $\mathbf{6}$ (2-back task, self-reported working memory and EF in daily life, emotional and external eating, pathological thoughts regarding food, body and weight, self-control and healthy eating).

The working memory training group consumed less snacks at a bogus taste test at post-measurement than control, which indicates a far transfer effect to control over eating behavior in a laboratory measure. Interestingly, the effect of working memory training was moderated by craving: participants in the experimental group who experienced a high craving ate less than participants in the control condition with high craving, whereas for those experiencing low craving, no differences between groups were found. This is in line with dual process models, as these findings indicate that the training is most effective in reducing intake when there is a strong impulse to consume food (i.e., craving) that requires self-regulation (Friese et al., 2008). Contrary to our expectations, both the experimental and control group lost a statistically significant though clinically modest amount of weight (i.e., both conditions lost on average $1.26 \%$ 
of their BMI at posttest, $1.52 \%$ after one month and $1.61 \%$ after six months relatively to baseline), without any differences between conditions.

The working memory training improved eating behavior on the short-term, resulting in less food intake in a laboratory bogus taste test, but no long-term effects could be demonstrated. The reduction in intake was small, but the experimental manipulation did influence eating behavior significantly. In the literature, long-term effects of training of EF have not been consistently demonstrated, and EF training applied to other populations also endorses this lack of long-lasting effects of EF changes as a major issue (Diamond \& Lee, 2011; Melby-Lervåg \& Hulme, 2013). Unfortunately, it seems that once the practice ends, the benefits diminish again, in line with results of chapter 6 . Future research should find out whether booster sessions possibly can strengthen training effects, and if so, when, how often and in which frequency they should be provided. For clinical significance and thus clinical applications, more intensive training sessions might be needed, and in the most ideal world this WM training would be added to other effective interventions.

\section{Manipulating cognitive control: Cognitive flexibility}

The association between the core EF shifting and eating behavior is relatively little studied. To shed more light on the association of shifting with eating behavior, in chapter $\mathbf{5}$ we studied in a two-study experimental design the influence of cognitive flexibility exercises on shifting performance and eating behavior. Results of study 1 revealed initial promising results following the manipulation, as performance on both the Wisconsin Card Sorting Test (WCST) and TMT was marginally significantly better in the experimental group relative to the control group. However, no differences in eating behavior between conditions could be discovered. In study 2, we made some changes to the design by adding a baseline measure of shifting and we extended the manipulation to two sessions. We focused on an undergraduate sample with an explicit desire to restrict food intake. Results for shifting on the WCST and TMT could not be consistently replicated in study 2 , as the experimental group completed more categories on the WCST, but no differences on the TMT were discovered, and again no results on eating behavior were found. It seems that our manipulation was not successful in heightening shifting skills or influencing food intake, indicating a lack of transfer of shifting improvements, in line with the current debate on the transferability of EF training to non-trained skills (e.g. Diamond \& Ling, 2016).

Importantly, we did not apply the full therapy in our research, but focused on some of the cognitive flexibility exercises of Cognitive Remediation Therapy (CRT), as our participants were healthy students with a weight loss goal instead of a clinical sample. A recent study demonstrated that eight sessions of CRT adapted for obesity (CRT-O) added 
to a behavioral weight loss intervention did result in improved shifting as measured with the TMT and WCST relative to a control group who only received the behavioral weight loss treatment (Raman, Hay, Tchanturia, \& Smith, 2017). The experimental group also reported reduced binge eating and lost more weight at the 3-month follow-up, whereby changes in the WCST predicted changes in weight. Besides exercises that facilitate cognitive flexibility, CRT-O focuses in face-to-face therapy sessions on reflective thinking techniques linking thinking style to dietary and physical activity, problem solving and attention to detail. As the results from this trial seem promising, future research could focus on replication of these results and study which elements of CRT influence shifting and weight loss. To conclude, the role of shifting in eating behavior remains unclear, as current results indicated no important role of shifting in eating behavior and obesity, and enhancing cognitive flexibility could be both beneficial (e.g. Raman et al., 2017; Evelyn Smith, Hay, \& Raman, 2014) or disadvantageous for initial weight loss (see results of chapter 2).

\section{Some theoretical and methodological considerations Discussing results in the light of dual process theories}

Successful self-regulation requires 1) a valued standard and actively monitoring of this standard, 2) the motivation to reduce discrepancy between the current state and this aimed for standard, and finally, 3) sufficient capacity to achieve this despite temptations and obstacles along the road (Hofmann et al., 2012). Control is driven by someone's desires and goals, thus, in short, control is always motivated (Botvinick \& Braver, 2015). According to the Reflective-Impulsive model, impulsive preferences no longer determine behavior and goals are more easily translated into behavior with stronger EF (Hofmann, Friese, \& Roefs, 2009; Hofmann et al., 2008). Thus, training EF to extend capacity will be of little use if someone has no clear goal to strive for, or no motivation to apply this extended capacity to get there. Importantly, while a dual-systems approach was used to inform design of experiments and interpretation of results, the role of the impulsive system was not a focus of the research reported in this dissertation. Instead, we assumed that our samples of participants would experience a conflict between the reflective and impulsive system. Therefore, participants in chapter 5-study 2 and chapter 6 were selected based on a pre-existing goal (i.e., intention) to either restrict intake or lose weight, and in chapter $\mathbf{6}$, we also screened participants to have sufficient motivation to work for this weight loss. However, the conflict between both systems was not explicitly measured, thus we have not directly tested whether better EF do lead to a decrease of impulsive influences on eating behavior. 


\section{Targeting general top-down mechanisms versus food-specific training}

There has been an extensive body of research conducted on training of inhibition, resulting in two recent meta-analyses establishing its effectivity in influencing eating behavior and weight loss (Allom, Mullan, et al., 2016; Jones et al., 2016). It is concluded that training with the GNG is more effective than training with the SST. Interestingly, the underlying mechanism of this GNG intervention seems more based on weakening the automatic system, instead of strengthening the executive system (Allom, Mullan, et al., 2016; Veling, Lawrence, Chen, van Koningsbruggen, \& Holland, 2017). Thus, the GNG and SST influence behavior via different underlying mechanisms: whereas training with the GNG influences behavior via bottom-up, automatic processes, training with the SST seems to influence behavior via top-down inhibition (Houben, Havermans, Nederkoorn, \& Jansen, 2012; Verbruggen \& Logan, 2008), of which the former seems most successful in reducing overeating.

Research suggest that training effects of the food-specific GNG are based on associative learning processes, which entail the formation of new associations between appetitive cues and inhibition (i.e., stopping) (Best, Lawrence, Logan, McLaren, \& Verbruggen, 2016; Houben \& Jansen, 2015), that it also seems to cause devaluation of those cues (Veling, Aarts, \& Stroebe, 2013), and results in reduced motor excitability when those cues are encountered (Verbruggen, Best, Bowditch, Stevens, \& McLaren, 2014). Thus, training of general inhibition is not as effective at influencing eating behavior as behavior-specific training, which also seems to apply to episodic future thinking (chapter 4). This suggests that their food-specific nature makes these 'interventions' effective. The shifting manipulation and working memory training as described in chapter $\mathbf{5}$ and $\mathbf{6}$ were focused on improving general top-down processes instead. We expected that the improvement of top-down control would transfer to other untrained tasks supported by this same EF network (Spierer, Chavan, \& Manuel, 2013), which unfortunately could not be consistently demonstrated. Inhibition research and the promising findings of food-specific episodic future thinking as reported in the current dissertation therefore suggest interesting avenues for further research. Training of food-specific or diet-related working memory and shifting could potentially increase effects of these EF manipulations. As EF should be activated when someone experiences a self-control conflict (e.g. encountering a tempting food situation while being on a diet), it would be interesting to have participants train their EFs while experiencing this 'hot' state, to potentially heighten transfer effects to tempting situations in daily life. Then, general capacity would not be improved per se, but the association between food-related cues and the use of EF would be strengthened (Jones, Hardman, Lawrence, \& Field, 2017). 


\section{Implications and future directions}

The current worldwide prevalence of overweight and obesity can be considered alarming. A clear understanding of the causes of obesity could eventually guide the development of more effective interventions to target obesity. To date, few studies have examined the impact of EF training over time in an obese population, even though the present literature points out the important role of EF in self-regulation and its great potential as addition to weight loss interventions (Houben, Nederkoorn, \& Higgs, 2017). Evidence for the transfer of EF training to non-trained tasks is rather scarce and comes from a wide variety of experimental paradigms. In the second part of this dissertation, we used an experimental method to test if intervening on EF leads to less overeating. Our results provide inconsistent evidence and a mixed picture of the effectiveness of EF training, though food-specific episodic future thinking and working memory training revealed some preliminary promising effects on food intake. Reducing intake of snacks contributes to a negative energy balance, which eventually will lead to weight loss. Continuing research to expand our understanding of the exact role of EF in obesity seems an important next step, though it is clear that considerable steps are needed before clinically relevant effects on behavior change can be achieved. Ultimately, future research should measure the influence of a certain manipulation on food intake in daily life. Modern technologies such as mobile applications give rise to whole new possibilities of easy measuring options in daily life, for instance with ecological momentary assessment, which provides a real-time assessment of eating behaviors (Boh et al., 2016).

Current results are not only interesting for obesity-related research, but could also guide further research on other health behaviors (Hall \& Marteau, 2014; Hofmann et al., 2012) and impulse-control disorders (e.g. Koffarnus et al., 2013). For instance, alcohol and drugs abuse have been associated with weak EF (Bechara, 2005; Bechara \& Martin, 2004; Noël, Bechara, Dan, Hanak, \& Verbanck, 2007), and deficits in EF have also been consistently associated with attention-deficit/hyperactivity disorder (ADHD) (Willcutt, Doyle, Nigg, Faraone, \& Pennington, 2005). Steeper delay discounting has been related to binge eating (Manasse et al., 2014; Manwaring, Green, Myerson, Strube, \& Wilfley, 2011; Svaldi, Brand, \& Tuschen-Caffier, 2010), ADHD (Barkley, Edwards, Laneri, Fletcher, \& Metevia, 2001), pathological gambling (Dixon, Marley, \& Jacobs, 2003), smoking (Baker, Johnson, \& Bickel, 2003), drinking (Vuchinich \& Simpson, 1998) and substance abuse (Madden, Bickel, \& Jacobs, 1999). Weak EF and high levels of delay discounting seem a central feature and trans-disease process underlying maladaptive behavior involved in impulse-control disorders. 


\section{TO CONCLUDE}

Based on the data as described in this dissertation, we conclude that 1) Individuals with obesity display weaker general and food-specific inhibition than healthy weight controls, and they report weaker EF in daily life. 2) Better behavioral working memory and self-reported inhibition at baseline are predictive of a higher percentage of weight loss following multidisciplinary treatment. 3) Unhealthy eaters seem less futureoriented regarding their food choices, and in order to be predictive of eating behavior, measures of time orientation should be tailored to eating behavior and health. 4) Episodic future thinking during decision making can reduce delay discounting, whereas only food-related episodic future thinking reduces food intake. Future research should focus on whether episodic future thinking entails a trait or state effect and how it can be transformed into an intervention with long-lasting effects. 5) The role of shifting in eating behavior and obesity could not be demonstrated. 6) Working memory training seems to improve eating behavior at the short term, though gains are short-lived and the added value of working memory training as an intervention to promote weight loss could not be established. Future research should continue to study the precise role of working memory in eating behavior and weight loss, to optimize potential effects of working memory training. 7) At the moment, evidence for transfer of EF training to healthy eating and weight loss remains scarce. However, as strikingly stated by Jones et al. (2017), there seems to be an absence of evidence concerning the potential of EF training in eating behavior and weight loss, instead of evidence of absence, though note that research in other domains strongly questions the existence of transfer effects of general top-down EF training to daily life (e.g. Diamond \& Ling, 2016). Based on evidence of the effectiveness of food-specific inhibition training (Allom, Mullan, et al., 2016; Jones et al., 2016) and current results pointing out the effectiveness of food-specific episodic future thinking, future research could focus on the development and testing of foodspecific EF training instead of strengthening general control mechanisms, to potentially support transfer effects to eating behavior and weight loss. 



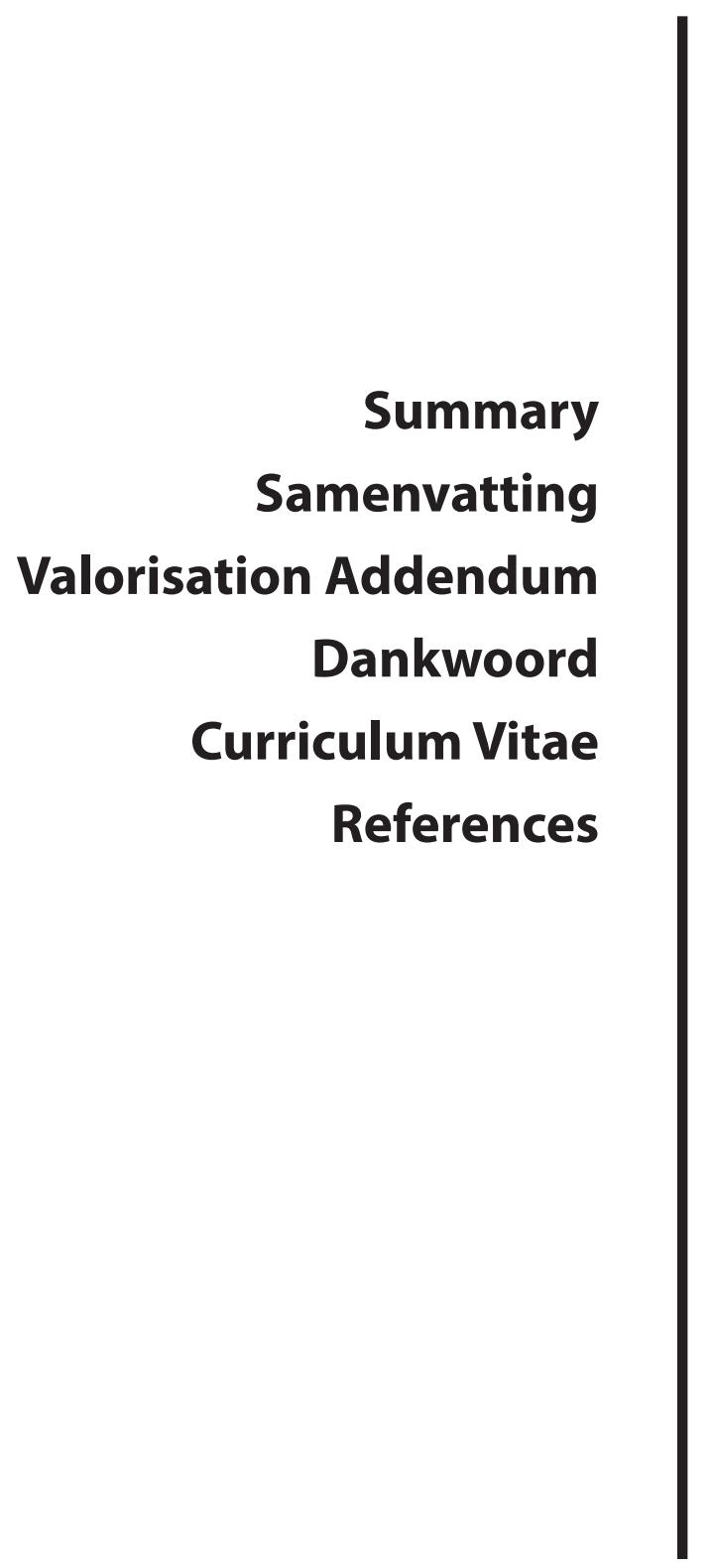





\section{SUMMARY}

The current dissertation addressed the role of executive function (EF) and time orientation in eating behavior, obesity and weight loss. Prevalence of obesity is high nowadays and current lifestyle weight loss treatments, which focus on modifying eating and physical activity habits to achieve a negative energy balance, lack long term effectiveness. It seems that intentions to lose weight do not always lead to an actual change in behavior. Dual process theories such as the Reflective-Impulsive model and the Competing Neurobiological Decision Systems theory state that our behavioral decisions rely on the interplay of two cognitive systems: the top-down reflective system, which promotes controlled, goal-oriented behavior, and the bottom-up impulsive system, which is responsible for automatic, associative behavior. Effective self-regulation is necessary to halt automatic impulses in favor of top-down goal-oriented behavior, and effective self-regulation might help to make healthy food choices in the current obesogenic environment.

Chapter 1 introduces EF as important factor in weight management and eating behavior. EF has been identified as one of the boundary conditions underlying the strength and dominance of the reflective system. There are three main facets of EF generally distinguished in the literature: inhibition, working memory and shifting. Another important EF-related construct related to cognitive control is time orientation. Conceptualizations of time orientation of interest in the current dissertation were Consideration of Future Consequences (CFC), which is the tendency of individuals to focus in general on immediate or future consequences of their current behavior, and delay discounting, which is the extent to which the value of a reinforcer decreases as a function of time. The goal of this dissertation was to investigate the role and trainability of EF and time orientation in eating behavior and weight loss. Our first aim was to establish the association of EF and time orientation with eating behavior and weight loss. EF and time orientation seem amendable to training, though research in the domain of eating behavior and EF training is scarce. Therefore, the second aim of this dissertation was to study the trainability of EF in experimental designs.

Chapter $\mathbf{2}$ describes a study in which individuals with obesity and healthy weight controls were compared on the three main facets of EF and delay discounting. Additionally, we examined whether EF and delay discounting predicted weight change for the individuals with obesity after six months participation in a multidisciplinary weight loss program. Results revealed that the individuals with obesity displayed weaker behavioral inhibition on the general and food-specific Stop-Signal Task (SST), relative to the healthy weight controls. They also reported weaker working memory, inhibition and shifting skills in daily life, though note that the results for shifting were only marginally significant. Moreover, results revealed that EF was indeed predictive of 
weight loss: Better performance on a behavioral working memory task and better selfreported inhibition skills in daily life were associated with more weight loss. Shifting as measured with the Trail Making Test (TMT) and delay discounting were neither related to BMI nor weight loss.

Chapter 3 describes a study examining general and food-specific measures of time orientation in an online questionnaire provided to a community sample. Results showed that a food-specific measurement of time orientation (CFC-food) was associated with self-reported eating behavior, with more focus on immediate consequences and less focus on future outcomes being related to less healthier eating behavior. The general CFC scale and delay discounting for money were not related to eating behavior. The adapted delay discounting measure with snack food was also not related to eating behavior. We discuss that this adapted task does not seem to capture the right dilemma for an individual trying to lose weight (e.g., the choice between a small amount of food versus even more food later, instead of a small amount of food versus future weight loss). Based on these results, it was concluded that CFC should be tailored to the behavior at interest (e.g. food and health), and that manipulating time orientation could be an interesting target for intervention.

Therefore, in chapter 4, we examined whether a manipulation of time orientation would influence food intake, by shifting concern with immediate gratification to a more future-oriented outlook. Specifically, we tested a manipulation of episodic future thinking in a female student sample, and explored whether the effects of episodic future thinking on food intake could be enhanced by making the content of imagery foodrelated. Results showed that delay discounting could indeed be reduced by episodic future thinking, and that this effect was not determined by content: both general and food-related episodic future thinking reduced delay discounting relative to control. Only food-related episodic future thinking reduced food intake relative to food-specific control, in line with our hypothesis. However, as no association was observed between discount rate for money and food intake, it appears that this was not the mechanism underlying the effect of episodic future thinking on eating behavior. Current results suggest that food-specific episodic future thinking could be a promising technique to resist immediate gratification of palatable, high-caloric food.

In chapter 5, two studies are presented in which it was explored whether a cognitive flexibility manipulation would result in better shifting skills and heightened cognitive control over eating behavior. The manipulation consisted of paper-and-pencil exercises, inspired by exercises included in Cognitive Remediation Therapy (CRT). Results of study 1 showed that the experimental group outperformed the control group on the Wisconsin Card Sorting Test (WCST) and TMT following the manipulation; though note that results were only marginally significant. On eating behavior, no differences between 
conditions were discovered. In Study 2, two sessions were offered to undergraduate females who were pre-screened to have a goal to restrict food intake. The experimental group completed more categories on the WCST, though no differences on perseverative errors on the WCST or switch costs on the TMT were found. Also, no differences in food intake or the intention-behavior gap for snacking, fruit and vegetable intake were discovered. Thus, our manipulation did not influence food intake, and effects on shifting were neither very consistent nor strong. Results seem in line with the current debate in the literature stressing that only a near transfer can be achieved to tasks very similar to the trained tasks, though no transfer to more complex tasks or daily life.

In chapter 6, a gamified version of working memory training was offered to overweight and obese individuals with a desire to lose weight. It was investigated whether working memory training in combination with an online lifestyle intervention would lead to improved self-regulation and increased weight loss compared to a lifestyle intervention combined with sham training. Results showed that working memory could be improved via training (trained tasks), though working memory gains were short-lived, as the experimental group was equal in level to control again on the six month follow-up. The current study did not find evidence for transfer to a non-trained working memory measure (2-back working memory task), and there was also no evidence for transfer effects to daily life, as both the experimental and control group showed improvements on the secondary outcome measures (self-reported EF, self-control, eating style, eating psychopathology and healthy eating). On average, participants lost a small amount of weight, though the working memory training did not result in additional weight loss compared to lifestyle intervention with sham training. Importantly, participants in the experimental condition consumed significantly less food than participants in the control condition during a bogus taste test immediately following the working memory training. Thus, results provide some evidence that working memory training can improve working memory performance and eating behavior at the short term, though the long-term effectiveness of the current training could not be demonstrated. Further research could focus on how working memory training can be made more effective as an intervention to promote long term weight loss.

In chapter 7, the main findings are summarized and discussed per subpart of this dissertation, and methodological considerations and suggestions for future research are provided. The chapter ends with a conclusion regarding the main findings of this dissertation. In short, regarding the cognitive profile of obesity, we found that individuals with obesity displayed weaker inhibition skills (general and food-specific) and reported weaker EF in daily life relative to healthy weight controls. Better behavioral working memory was the strongest predictor of weight loss of individuals with obesity after six months participation in a multidisciplinary weight loss program. Unhealthy eating was 
associated with being less future-oriented and being more present-minded regarding food choices. Working memory and food-specific time orientation therefore seem interesting targets to influence eating behavior and weight loss. In the second part of this dissertation, we found that in order to reduce overeating, episodic future thinking should be behavior-specific. Working memory training improved eating behavior at the short term. As a next step, future research could focus on whether and how episodic future thinking and working memory training can be transformed into interventions with long-lasting effects. The role of shifting in eating behavior and obesity could not be unequivocally demonstrated. Thus, future research should continue to study effects of working memory training and episodic future thinking on eating behavior and weight loss. Previous research shows that food intake can successfully be influenced via foodspecific inhibition training, and it seems that this food-specificity makes inhibition training effective. This food-specificity also seems to apply to episodic future thinking. The development of food-specific or diet-related EF training provides fruitful directions for future research. 


\section{SAMENVATTING}

De prevalentie van obesitas is tegenwoordig alarmerend hoog. Huidige behandelingen voor gewichtsverlies, die zich met name richten op het veranderen van eetgewoonten en het verhogen van fysieke activiteit om zo een negatieve energiebalans te bereiken, blijken niet effectief op de lange termijn. Het lijkt erop dat goede voornemens om af te vallen niet altijd leiden tot een daadwerkelijke verandering in gedrag. Dualprocestheorieën stellen dat onze beslissingen afhankelijk zijn van het samenspel van twee cognitieve systemen: het top-down reflectieve systeem, dat verantwoordelijk is voor gecontroleerd, doelgericht gedrag en het bottom-up impulsieve systeem, dat verantwoordelijk is voor automatisch, associatief gedrag. In de huidige obesogene omgeving kan effectieve cognitieve controle mogelijk helpen om gezonde voedingskeuzes te maken en zo overeten te voorkomen.

Hoofdstuk 1 introduceert executief functioneren (EF) als belangrijke factor in gewichtsbeheersing en eetgedrag. EF ligt ten grondslag aan de sterkte en dominantie van het reflectieve systeem, en met sterkere EF kunnen we beter onze impulsen bedwingen. In de literatuur wordt over het algemeen over drie hoofdaspecten van EF gesproken: inhibitie, werkgeheugen en switchen (ook wel cognitieve flexibiliteit genoemd). Daarnaast lijkt de factor tijdoriëntatie belangrijk voor cognitieve controle. In het huidige proefschrift hebben we naar de volgende conceptualisaties van tijdoriëntatie gekeken: de overweging van toekomstige gevolgen (CFC), wat de neiging is van iemand om zich meer op onmiddellijke of toekomstige gevolgen van zijn of haar gedrag te richten, en delay discounting, wat de mate is waarin de waarde van een bekrachtiger afneemt naarmate deze later in de tijd verkregen wordt. lemand die vaker voor een kleinere, snellere beloning kiest in plaats van een grotere, maar later te ontvangen beloning wordt als meer impulsief gezien. Het eerste deel van dit proefschrift richt zich op de rol van EF en tijdoriëntatie bij eetgedrag, obesitas en gewichtsverlies. EF en tijdoriëntatie lijken verbeterbaar door training, hoewel onderzoek op het gebied van eetgedrag en EF training nog schaars is. Daarom richt het tweede deel van dit proefschrift zich op de manipulatie en trainbaarheid van EF in experimentele designs.

Hoofdstuk 2 beschrijft een onderzoek waarin ten eerste personen met obesitas en gezonde gewichtscontroles werden vergeleken op de drie hoofdfacetten van $\mathrm{EF}$ en delay discounting. De personen met obesitas namen vervolgens zes maanden deel aan een multidisciplinair programma gericht op gewichtsverlies. Ten tweede onderzochten we of we gewichtsverandering voor de personen met obesitas konden voorspellen aan de hand van EF en delay discounting. De resultaten toonden aan dat de personen met obesitas een zwakkere inhibitie vertoonden, zowel in het algemeen als specifiek gericht op snackvoedsel, ten opzichte van de gezonde gewichtscontroles. De personen met obesitas rapporteerden ook zwakkere inhibitie, een zwakker werkgeheugen en slechter 
switchen in het dagelijks leven, hoewel de resultaten voor switchen slechts marginaal significant waren. Bovendien toonden de resultaten aan dat EF inderdaad voorspellend was voor gewichtsverlies: betere prestaties op een werkgeheugentaak en betere zelfgerapporteerde inhibitievaardigheden in het dagelijks leven gingen gepaard met een groter gewichtsverlies.

Hoofdstuk 3 beschrijft een onderzoek waarin we algemene en eetspecifieke meetinstrumenten van tijdoriëntatie onderzochten via een online vragenlijst. We verwachtten dat de eetspecifieke vragenlijsten betere voorspellers zouden zijn van eetgedrag dan de algemene vragenlijsten. De resultaten lieten zien dat een eetspecifieke versie van de vragenlijst naar tijdoriëntatie (CFC-eet) inderdaad samenhing met eetgedrag, waarbij het meer bezig zijn met directe gevolgen en minder focus op toekomstige gevolgen samenhing met een ongezonder eetpatroon. De algemene meetinstrumenten vertoonden geen associatie met eetgedrag. Op basis van deze resultaten concludeerden we dat het manipuleren van tijdoriëntatie een interessant doelwit zou kunnen zijn voor interventie.

Vervolgens hebben we in hoofdstuk 4 een manipulatie van tijdoriëntatie onderzocht. We onderzochten of episodisch toekomstdenken de focus op onmiddellijke beloning kon verschuiven naar een meer toekomstgerichte blik. Daarnaast keken we of we eetgedrag konden beïnvloeden door de inhoud van deze toekomstbeelden eetgerelateerd te maken. De resultaten toonden aan dat delay discounting, het impulsief kiezen voor een kleinere, directe beloning in plaats van een grotere, uitgestelde beloning, inderdaad kon worden verminderd door episodisch toekomstdenken. Dit effect werd niet werd bepaald door de inhoud van de beelden: zowel algemeen als eetgerelateerd episodische toekomstdenken verminderde dit impulsieve keuzegedrag ten opzichte van de controlegroep. Echter enkel eetspecifiek episodisch toekomstdenken verminderde de snackinname in vergelijking tot de controlegroep, in lijn met onze hypothese. Resultaten tonen dus aan dat eetspecifiek episodisch toekomstdenken een veelbelovende techniek zou kunnen zijn om niet telkens te vallen voor de verleiding van de onmiddellijke beloning die smakelijk, hoog calorisch voedsel biedt.

In hoofdstuk 5 worden twee studies gepresenteerd waarin we onderzochten of een cognitieve flexibiliteitsmanipulatie zou leiden tot betere switchvaardigheden en verhoogde cognitieve controle over eetgedrag. De manipulatie bestond uit een aantal cognitieve flexibiliteitsoefeningen op papier. Resultaten van Studie 1 toonden aan dat de experimentele groep na afloop beter presteerde dan de controlegroep op twee bekende taken om switching oftewel cognitieve flexibiliteit te meten, hoewel resultaten slechts marginaal significant waren. Op eetgedrag werden geen verschillen tussen de condities ontdekt. In Studie 2 werden twee sessies aangeboden aan vrouwelijke studenten die probeerden om hun dagelijkse calorie-inname te beperken om zo af te 
vallen of op gewicht te blijven. De experimentele groep presteerde na afloop wederom beter op een van de switchtaken, maar op de tweede taak werden geen verschillen gevonden, en resultaten op de eerste taak waren niet erg sterk. Ook waren er wederom geen verschillen tussen de condities in eetgedrag. Onze trainingseffecten waren dus niet erg consistent of sterk, wat in overeenstemming lijkt met het huidige debat in de literatuur wat benadrukt dat enkel op taken die sterk lijken op de getrainde taken een verbetering kan worden bereikt door training, terwijl er geen verbetering op complexere cognitieve taken of in het dagelijks leven bereikt lijkt te worden.

In hoofdstuk $\mathbf{6}$ werd een game-variant van een werkgeheugentraining aangeboden aan mensen met overgewicht die de wens hadden om af te vallen. Er werd onderzocht of training van het werkgeheugen in combinatie met een online leefstijlinterventie tot verbeterde zelfcontrole en meer gewichtsverlies zou leiden in vergelijking tot een controlegroep, die een simpelere controletraining gecombineerd met een leefstijlinterventie ontving. De resultaten lietenzien dathet werkgeheugen verbeterd was na afloop van de training, hoewel deze verbeteringen in werkgeheugen van korte duur waren, aangezien de experimentele groep weer op hetzelfde niveau als de controlegroep zat tijdens de zes maanden follow-up meting. Het huidige onderzoek vond geen bewijs voor een overdracht van trainingseffecten naar een complexere niet-getrainde werkgeheugentaak, en er werd ook geen bewijs gevonden voor verbeteringen in het dagelijks leven. Gemiddeld verloren alle deelnemers een klein beetje gewicht, hoewel de werkgeheugentraining niet resulteerde in extra gewichtsverlies ten opzichte van de leefstijlinterventie gecombineerd met controletraining. Deelnemers in de experimentele conditie consumeerden echter significant minder snacks dan de controlegroep tijdens een zogenaamde smaaktest direct na de training. Deze bevindingen leveren dus enig bewijs dat een werkgeheugentraining werkgeheugenprestaties en eetgedrag op korte termijn kan verbeteren, hoewel de langetermijneffectiviteit van de huidige training niet kon worden aangetoond.

In hoofdstuk 7 worden de belangrijkste bevindingen per subdeel van dit proefschrift samengevat en besproken, we bespreken methodologische overwegingen en geven suggesties voor toekomstig onderzoek. Het hoofdstuk eindigt met een conclusie met betrekking tot de belangrijkste bevindingen van dit proefschrift. Met betrekking tot het cognitieve profiel van obesitas hebben we aangetoond dat personen met obesitas zwakkere inhibitie-vaardigheden vertonen (zowel algemeen als eetspecifiek) en ze rapporteerden zwakkere EF in het dagelijks leven ten opzichte van gezonde gewichtscontroles. Een beter werkgeheugen was de beste voorspeller van gewichtsverlies voor personen met obesitas na zes maanden deelname aan een multidisciplinair programma. Ongezond eetgedrag hing samen met minder toekomstgericht zijn en meer bezig zijn met directe gevolgen als het ging om 
voedselkeuzes en gezondheid. In het tweede deel van dit proefschrift constateerden we dat episodisch toekomstdenken gedragsspecifiek moet zijn om overeten te verminderen. Een werkgeheugentraining leidde tot een verbetering in eetgedrag op de korte termijn. De rol van switching in eetgedrag en obesitas kon niet eenduidig worden aangetoond. Toekomstig onderzoek zou zich kunnen concentreren op de vraag hoe episodisch toekomstdenken en werkgeheugentraining kunnen worden omgezet naar interventies met langdurige effecten. Uit eerder onderzoek blijkt namelijk dat eetgedrag met succes kan worden beïnvloed door inhibitietraining, en het lijkt erop dat het met name werkt als proefpersonen een training met eetstimuli in plaats van algemene stimuli volgen. Wij vonden in overeenstemming hiermee dat episodisch toekomstdenken zich ook specifiek op eetgedrag moet richten om snackinname te beïnvloeden. Dus, EF training specifiek gericht op eetgedrag en diëten lijkt een veelbelovende richting voor toekomstig onderzoek. 


\section{VALORISATION ADDENDUM}

\section{Creating value from science}

\section{Relevance}

The rates of overweight and obesity have doubled since 1980, and continue to rise rapidly (Finucane et al., 2011). It is estimated that at the moment around 1.9 billion adults are overweight, and approximately 600 million adults are considered obese (WHO, 2016). Some experts even talk about an epidemic. Obesity is associated with adverse health consequences as it places individuals at risk of cardiovascular diseases, various forms of cancer, diabetes mellitus type II and musculoskeletal disorders (Lim et al., 2013). It is also associated with serious psychological consequences such as a reduced quality of life (Uzogara, 2017).

There is a wide variety of diets and diet products available, all promising quick and easy weight loss. The problem, however, seems to lie for a large part in adherence to a diet. We live in a so-called obesogenic environment, an environment that promotes gaining weight and one that is not conducive to weight loss (Swinburn, Egger, \& Raza, 1999). Unhealthy, high-caloric foods are cheap and available in abundance, portion sizes have increased, and unhealthy products are aggressively marketed. Obviously, this makes it difficult to maintain a healthy lifestyle and to follow the rules of a particular diet or stick to diet products only. Traditionally, obesity has been considered as mainly a biomedical problem, though behavioral factors seem to play an important role in the etiology and maintenance of obesity (Jansen et al., 2009).

Current lifestyle treatment programs which focus on reduced intake and heightened physical activity do result in modest initial weight loss (around 8-10\%; Butryn, Webb, \& Wadden, 2011). However, only a small percentage of dieters (between $3 \%$ and $21 \%$, depending on the definition of 'successful weight loss') manages to keep the weight off in the long-term (Ikeda et al., 2005). Thus, it is clear that we need more effective interventions to combat this epidemic. This thesis provides a first step in the development of new trainings targeting the cognitive profile of obesity. In part one, cognitive weaknesses associated with unhealthy eating behavior and weight loss success were identified, and in part two, causal associations between these cognitive elements and eating behavior and weight loss were tested in experimental designs. As experimental research aimed at the training of executive function in the field of eating behavior and weight loss is still sparse, this thesis focused on a question that was, and still is, a very prominent one. 


\section{Target groups}

As the numbers of overweight and obese individuals are rising, the conducted studies were aimed at a large target group. We studied in this thesis a broad range of participants, with and without weight loss goals, within multiple age groups. The findings described in this thesis are relevant for individuals with overweight or obesity, individuals at risk, clinicians, researchers, research organizations and policy makers. The obesogenic epidemic places a high burden on health care providers, which leads to high costs for insurance companies and therefore for society. If we know more about the etiology of obesity, eventually treatment can be optimized and money can also be used more efficiently. The current manipulations developed for an obese, adult sample could also be adapted and studied in the treatment of other impulse-disorders (e.g. alcohol abuse, smoking) and in other age groups (e.g. children and elderly).

\section{Activities and products}

Results of the research in this thesis have been presented at scientific conferences, but also at more applied, clinical conferences, for instance, the Association of Cognitive Behavioral Therapy in Veldhoven, the Netherlands, and the Workshop Congress of Clinical Psychology and Psychotherapy in Chemnitz, Germany. These conferences are attended by both researchers and clinical therapists and therefore these events facilitate contact between science and clinical practice: therapists can learn about new scientific developments, and scientists can get direct feedback from the clinical practice.

It takes time before treatment can be applied into the clinic. The extensive collaboration of our eat-lab with clinical institutions makes it possible to discuss and implement results at an early stage of the development. Co-Eur Centre for Obesity and Eating Disorders, a multidisciplinary weight loss clinic, was involved in the execution of the research described in chapter 2 , and the staff of Co-Eur as well as their clients will be informed about our outcomes.

It is not only important to inform clinicians, but also to the general population. Besides scientific publications, we also shared our knowledge with a more broad audience. A Dutch overview article was published in the online peer-reviewed journal The Inquisitive Mind, which is targeted at the scientifically interested lay person. This article is currently being translated for the German edition of the Inquisitive Mind. In the summer of 2016, a public event was organized, during which the research of the eat-lab was presented to the general public. Visitors could for instance get hands-on experience with and provide us with feedback about the gamified working memory training described in chapter 6 .

Teaching to students of Maastricht University was also part of this project. Many students have gained experience in the set-up and execution of scientific research by 
assisting with the research described in this dissertation. Lectures about this project and related topics have been provided to the (upcoming) students of Maastricht University and the University College Venlo. Also children from elementary schools and teens from secondary schools had a first acquaintance with science and our eating behavior research in initiatives from Maastricht University like KidzCollege and Class Days.

\section{Innovation}

Many studies show that individuals with obesity have less effective executive functions. The first innovative step is that we tried to train these functions and thereby demonstrate the causal connection, to eventually reduce obesity rates. In a number of ways this has been studied by other researchers in recent years (e.g. there is a large field of research which focused on inhibition training), but we looked for new ways to train and improve executive functioning by training of working memory, shifting and episodic future thinking.

A second innovation is in the way the training is being delivered. In chapter 6 , we made use of elements of 'gamification'. This entails the use of game-elements in a nongame context (i.e., the classic cognitive training paradigm) to motivate the user to conduct the training and increase his or her user activity (Deterding, Dixon, Khaled, \& Nacke, 2011). Earlier research shows that cognitive tasks can be successfully transformed into game format without losing their scientific value (Lumsden, Edwards, Lawrence, Coyle, \& Munafò, 2016). Thus, by adding game-elements, the scientific value of the training is retained, though the execution becomes more fun (Boendermaker, Prins, \& Wiers, 2015). We put a game shell around the classic online working memory training paradigm, and added external rewards. Unfortunately, current results provide mixed evidence for the effectiveness of our gamified working memory training in weight loss, and drop-out numbers of our gamified version were equal to previous research studying the classic training paradigm (Houben, Dassen, \& Jansen, 2016). Thus, future research should continue to study working memory training in experimental designs, before its potential as a tool for clinical practice can be established.

\section{Schedule and implementation}

The cognitive tasks and trainings that were developed in the context of this thesis, in collaboration with the Maastricht University Instrumentation department, are still available and usable. A major advantage of having such tasks and modules available within the university is that future research projects do not have to develop a cognitive training from scratch or rely on and invest in commercially available alternatives. Our tasks and trainings are currently used for multiple studies. For instance, within the Eatwell collaboration, a study testing the classic working memory training paradigm 
within COPD patients is currently being conducted by our colleagues of the Faculty of Health, Medicine and Life Sciences.

The current work has been part of the mission of the eat-lab to eventually develop more effective treatments for obesity. Current results will be used in follow-up research to continue to study the role and trainability of executive functioning in eating behavior and weight loss, as it is clear that considerable steps are needed before clinically relevant effects on behavior change can be achieved. Eventually, web-based training paradigms can become easily and cost-effectively tools to be used by obesity clinics in treatment, as participants can train at home or anywhere they want on their tablet or smartphone. It is also possible to make cognitive trainings available to the general public in the future via for instance the development of an app. 


\section{DANKWOORD}

Vier jaar verder en aardig wat ervaringen rijker, ging er een proefschrift naar de drukker! Het is nog steeds een beetje onwerkelijk dat dit boekje met mijn naam erop nu voor me ligt. Dit heb ik natuurlijk niet in mijn eentje gedaan. Ik heb de afgelopen vier jaar veel bijzondere mensen aan mijn zijde gehad, en ben ieder van hen zeer dankbaar voor hun fantastische gezelschap, inspiratie en vertrouwen in mij.

Graag een speciaal woord van dank aan een aantal personen. Om te beginnen natuurlijk mijn geweldige team van (co)promotoren:

Katrijn, dit proefschrift heeft veel aan jou te danken. Je gaf me vanaf het begin veel vrijheid en vertrouwen, en stond altijd klaar om mee te denken. We waren een goed team. Als ik weer eens in de stress zat en het allemaal niet meer zag zitten, was een gesprek met jou precies wat ik nodig had om weer in te zien dat het allemaal best wel goed zou komen. We konden alle kanten op, jouw zegen had ik, gewoon doen. Met behulp van jouw altijd spot-on feedback wist ik deze eindversie voorspoedig af te ronden. Fijn dat we niet alleen over werk praatten, maar je ook aandacht voor mij als persoon had. Als wetenschapper zijn je nuchtere instelling gecombineerd met een kritische blik een groot voorbeeld voor mij. Bedankt voor alles!

Anita, jouw optimisme en enthousiasme maken de eetgroep tot een aangename werkomgeving. Als promotor liet je mij heel vrij, maar hielp je wel de nodige knopen door te hakken tijdens onze overlegmomenten. Je leerde me mijn data naar waarde te schatten, 'promising', en ik kon altijd rekenen op je kritische blik bij het schrijven van mijn artikelen, waardoor er na jouw feedback plots kort en krachtig precies stond wat ik in gedachten had, maar toch net niet helemaal op papier had gekregen.

Sjaan, ons gezamenlijke Marble-project tijdens mijn studie heeft mijn interesse in (eet)onderzoek gewekt. Je deur stond altijd open voor vragen, en een opmerking of suggestie van jou sloeg altijd precies de spijker op de kop, bedankt daarvoor.

Een heel groot dankjewel aan mijn fantastische paranimfen:

Sieske, roomie en paranimf, ja wat moet ik zeggen...er was direct een klik, een gelijkgezind persoon! Samen hadden we het altijd heel gezellig, maar wisten we elkaar ook te motiveren tot productief zijn. Zo'n 2496 koffiemomentjes was je schatting he, en gelukkig still counting! Iris, mijn andere roomie en paranimf. Je kwam, en het was of je er altijd al was. Bedankt voor je luisterend oor, goede adviezen, je vertrouwen in mij en gewoon je gezelschap. Mijn supportbra's, door jullie kwam ik iedere dag weer met plezier naar kantoor! 
Anita, Carolien, Sjaan, Katrijn, Remco, Anouk, Eva, Janneke, Sandra, Anne, Jessica, Ghislaine, Karolien, Peggy, Bastiaan, Sarah, Bart, Sieske, Iris, Eric, Valerie, Yu, Leonardo en Lotte, oftewel de eetgroep. Het is enorm fijn om zo'n grote groep collega's te hebben om mee te brainstormen. Ik heb de vele gezellige Eatmeets, etentjes, borrels, congressen samen en Braindays enorm gewaardeerd!

Anne, ook een speciaal woord van dank aan jou, en wat fijn dat je in mijn corona plaats wilt nemen. Je gaf me altijd het gevoel dat je in mij geloofde, wat mij veel vertrouwen heeft gegeven. Met jou aan het roer gaat de eetgroep een mooie toekomst tegemoet.

Ik wil ook graag de leden van mijn beoordelingscommissie bedanken voor het lezen en beoordelen van mijn proefschrift: Prof. Annemie Schols, Prof. Caroline Braet, Prof. Rob Holland, Dr. Petra Hurks en Prof. Gerjo Kok. Bedankt dat jullie daarnaast ook plaats willen nemen in mijn corona.

Een speciaal woord van dank aan Eatwell, het interfacultaire samenwerkingsverband dat mijn PhD-project mogelijk heeft gemaakt. Ik hoop dat er nog veel mooie studies uit dit project voortkomen!

Zonder proefpersonen was er geen onderzoek mogelijk geweest. Via deze weg dan ook een groot dankjewel aan alle deelnemers van mijn studies in de afgelopen jaren. Een speciaal woord van dank voor Dr. Erik Aller en Daisy Jeurissen die toestemming gaven en mij hielpen om mijn studie beschreven in hoofdstuk 2 bij CO-EUR uit te voeren. Daarnaast wil ik ook graag mijn fantastische stagiaires en student-assistenten bedanken die geholpen hebben bij de dataverzameling van mijn studies: Johanna, Dennis, Kyra, David, Berber, Laura, Lianne, Manon en Hajar, jullie zijn stuk voor stuk toppers!

Jannette van Beek, bedankt dat je de CFC-food en Nederlandse vertaling met mij deelde, en leuk dat we af en toe mailden, ondanks dat ik je nooit in het echt heb ontmoet voel je een beetje als een collega.

Richard en Michiel, jullie hulp was onmisbaar in dit project, bedankt voor het meedenken en programmeren van het prachtige Eatwell-spel, de buffettaak, en de gebruikte computertaken. Michiel, bedankt dat ik altijd mocht binnenlopen als er iets niet helemaal volgens plan verliep, je had altijd tijd voor me en kwam in een mum van tijd met een oplossing. Richard, jouw creatieve input was van grote waarde, zonder jou had het er nooit zo prachtig en professioneel uitgezien. Ook bedankt voor de gezellige praatjes in de koffiehoek.

Lieve Anne, bedankt voor het ontwerpen van de cover, hij is prachtig geworden! 
Jessie, Marionne en Carolien, bedankt voor jullie hulp met allerlei praktische zaken en de gezellige gesprekken, ik ging altijd weer vrolijk weg bij het secretariaat. Lindy, bedankt dat je altijd alles wist als er iets geregeld moest worden. Eva, bedankt voor de hulp bij de afronding en je goede advies.

Prof. Frans Verhey en Prof. Marjolein de Vugt, bedankt dat jullie mij hebben gekozen voor deze functie als trainingsmanager bij het Alzheimer Centrum Limburg, ik kijk al uit naar onze samenwerking! Ook alle leden van INDUCT, INTERDEM Academy, Alzheimer Centrum Limburg en de afdeling Psychiatrie en Neuropsychologie, bedankt voor jullie hartelijke welkom, waardoor ik me direct thuis voelde op mijn nieuwe werkplek.

Mijn allerliefste mede-aio's, met wie ik een aanzienlijk deel van mijn tijd heb doorgebracht op werk, maar ook daarbuiten, nog een speciaal woord van dank aan jullie:

Karolien, wie weet, als ik bij mijn eerste voorstel was gebleven, was ik hier nooit geweest. Je kwam me op het begin gezelschap houden op mijn grote, maar toch wel wat eenzame kantoor. Je uitspraak 'je bent kapitein van je eigen schip' was spoton, mede daardoor heeft mijn proefschrift een inhoud gekregen waar ik helemaal achter sta. Peggy, je was een groot voorbeeld voor mij, je liet me zien hoe je op een gebalanceerde manier een promotietraject doorloopt, en bent ook gewoon een heel leuk persoon. Bedankt dat je de Peggy-love appgroep met me wil delen, laten we onze aio-reünies erin houden! Astrid, er was direct een klik, je bent met recht een echte collega-vriendin. Ghislaine, bedankt voor je advies in de afrondingsfase, je gaf me altijd het vertrouwen dat ik dit ook kon. Lotte, wat waren wij een topteam als organisatoren van de 'obesitas dag' in Geleen. Bastiaan, ik denk dat het langzaam tijd wordt voor ons volgende escape-avontuur. Thomas, let's buy a new 10-rittenkaart, to continue our bouldering adventures. Clare, thank you for being so enthusiastic about my studies, you really made me believe in my work! Thank you for proofreading my first manuscript as a native speaker. René, Vanessa, and Shannon, you all shared the office with me for a while and definitely made work more fun. Anke, jij kwam erbij toen ik alweer bijna wegging, fijn dat we nog even samen hebben kunnen werken. Ik kom graag nog eens buurten (of borrelen) op mijn oude kantoortje. Bram, bedankt voor de gezellige lunches, het was fijn om een mede-aio vanuit Rolduc te hebben.

WC'ers (WC=Writing Club), toppers, dankzij jullie is dit proefschrift ook echt geschreven. Onze wekelijkste meetings gaven structuur aan mijn schrijven. Jessica, thank you for being such an inspiring writing-mama!

Collega's van CPS: bedankt voor de leuke gesprekken en gezellige lunches in de koffiehoek. Maartje, vanaf dag 1 zaten we al samen in de bus, en nu nog steeds op weg 
naar mijn nieuwe werkplek 100m verderop. Van collega's tot wekelijkse hardloop- en SuperHIIT-maatjes; ik hoop dat we dit in de toekomst ook vol kunnen houden! Charlie, de ezeltjes staan nog steeds op de agenda, bedankt voor alle gezellige intermezzo's op de $3^{\mathrm{e}}$.

Mede EPP-ers, bedankt voor de gezellige workshops (inclusief heerlijke diners en glazen badkamerdeuren) in Heeze. Speciaal woord van dank aan Kai, voor de altijd gezellige spelletjesavonden bij EPP en ook daarbuiten.

Tot slot wil ik mijn lieve vrienden en familie bedanken voor hun support, en gewoon dat jullie er altijd voor mij zijn. In het bijzonder:

Loes, Claudia, Nadine en Nathalie, vanaf week 1 van onze studie waren we een groepje. Onze wekelijkste koffiedates zorgden dat ik altijd mijn frustraties kwijt kon, maar ook mijn highlights met jullie kon delen. Bedankt voor jullie vriendschap, ze zeggen dat de vriendschappen die je op de universiteit opdoet voor het leven zijn! Claudia, bedankt voor je enthousiasme en interesse, je voelde een beetje als mijn extra kamergenoot gedurende de afgelopen vier jaar (op afstand en ook 1x in het echt). Loes, onze geschiedenis gaat nog verder terug, bedankt dat je altijd voor me klaar staat, ik heb grote bewondering voor je als persoon. Dat ik vorig jaar je getuige op jullie bruiloft mocht zijn, vond ik heel speciaal.

Mariza, bedankt voor je nuchtere blik en oprechte interesse. Daarnaast ben ik gewoon heel bij met onze vriendschap. Dat we nog maar veel koffies en cocktails mogen drinken terwijl onze mannen weer eens over hun fantastische voetbalteam kletsen...

Yoeri, grote broer, ja misschien valt er op dit moment alsnog het kwartje bij een aantal personen dat wij familie zijn. Het was erg fijn om onderwijservaringen uit te wisselen en samen in de uni-wereld rond te lopen. Je onuitputbare enthousiasme en ideeën zijn soms overweldigend, maar uiteindelijk altijd zinnig. Je hebt me al vaak aan het denken gezet en gezorgd dat ik mijn kansen grijp en dromen najaag, bedankt daarvoor!

Papa en mama, het is moeilijk in woorden te vatten, door jullie veilige basis ben ik de persoon kunnen worden die ik nu ben. Ik kan altijd bij jullie terecht, gewoon bedankt voor alles!

Stephan, last but not least, deze these heeft het nodige aan jou te danken. Mijn thuisfront, rots in de branding. Samen met onze kippetjes en konijnen hebben we een fijn thuis opgebouwd in Partij. Bedankt dat je me altijd hebt aangehoord, zelfs midden in de nacht als ik plotseling een inval had. Je was mijn klankbord voor de nodige ideeën en hielp me relativeren. Met een door jou gezet kopje koffie in de ochtend kon ik er weer vol goede moed tegenaan. Ik ben gewoon heel blij met jou, bedankt dat je er altijd voor me bent! 


\section{CURRICULUM VITAE}

Fania Cher Marie-Louise Dassen was born in Heerlen (the Netherlands) on March 31, 1990. She graduated from College Rolduc in Kerkrade in July 2008, after which she started her Bachelor Psychology and Neuroscience at Maastricht University. She participated in the Maastricht Research Based Learning Program (MaRBLe), during which she studied impulsivity in the presence and absence of hunger in primary school children, under the supervision of Dr. Nederkoorn. She received a Top 3\% award in her first and second year of the Bachelor and graduated cum laude in July 2011. She started the Master Health Care Psychology at the Radboud University Nijmegen and specialized herself in Developmental Psychology. During her Master she did a clinical internship at Bureau Iris in Maastricht and obtained her diagnostic certificate (BAPD). She also conducted a research internship in which she studied intercultural differences in temperament and behavioral problems in children, under the supervision of Dr. Beijers. She received her Master's degree in December 2012, and subsequently, she worked a year as a team coordinator and care giver for children and elderly with mental and physical disabilities. In April 2014, she started her PhD project at the Department of Clinical Psychological Science at Maastricht University, funded by the interfaculty program Eatwell. She studied cognitive control interventions for obesity under the supervision of Prof. Jansen, Dr. Houben and Dr. Nederkoorn. During her PhD project, she obtained her University Teaching Qualification. Since April 2018, Fania works as Training Manager for INTERDEM Academy and INDUCT - two innovative European training networks on dementia care research and technology, at Alzheimer Centre Limburg, Maastricht University. 


\section{PUBLICATIONS AND PRESENTATIONS}

\section{Peer reviewed publications}

Dassen, F.C.M., Houben, K., Allom, V., Jansen, A. Self-regulation and obesity: The role of executive function and delay discounting in the prediction of weight loss (in press, Journal of Behavioral Medicine).

Dassen, F.C.M., Houben, K., Van Breukelen, G.J.P., \& Jansen, A. (2018). Gamified working memory training in overweight individuals reduces food intake but not body weight. Appetite, 124, 89-98. https://doi.org/10.1016/j.appet.2017.05.009

Dassen, F.C.M. (2017). Train je brein slank! Een cognitieve benadering voor gewichtsverlies. In-Mind Nederland. Retrieved from http://nl.in-mind.org/article/trainje-brein-slank-een-cognitieve-benadering-voor-gewichtsverlies.

Houben, K., Dassen, F.C.M., \& Jansen, A. (2016). Taking control: Working Memory training in overweight individuals increases self-regulation of food intake, Appetite, 105, 567-574. https://doi.org/10.1016/j.appet.2016.06.029

Dassen, F.C.M., Jansen, A., Nederkoorn, C., Houben, K. (2016). Focus on the future: Episodic future thinking reduces discount rate and snacking. Appetite, 96, 327-332. https://doi.org/10.1016/j.appet.2015.09.032

Nederkoorn, C., Dassen, F.C.M., Franken, L., Resch, C., Houben, K. (2015). Impulsivity and overeating in children in the absence and presence of hunger. Appetite, 93, 5761. https://doi.org/10.1016/j.appet.2015.03.032

Dassen, F.C.M., Houben, K., Jansen, A. (2015). Time orientation and eating behavior: unhealthy eaters consider immediate consequences, while healthy eaters focus on future health. Appetite, 91, 13-19. https://doi.org/10.1016/j.appet.2015.03.020

\section{Other manuscripts}

Dassen, F.C.M., Houben, K., Jansen, A. Manipulating cognitive flexibility: Can we change eating behavior through training of executive control? (ready for submission).

\section{Presentations at (inter)national conferences and symposia}

Dassen, F.C.M. (2017). Gamified working memory training reduces food intake but not body weight. Oral presentation at the Summer School 2017: Cognitive Control and Consciousness: Behavioural and Neural Mechanisms, organized by the Swiss Graduate School for Cognition, Learning, and Memory, Weggis, Switserland. 
Dassen, F.C.M., Houben, K., Van Breukelen, G.J.P., \& Jansen, A. (2017). Cognitive control and eating behavior: applying a gamified working memory training to the domain of weight loss. Oral presentation given as part of the symposium Binge-Eating-Störung und Adipositas: Aktueller Forschungsstand zur Behandlung at the Workshop Congress Clinical Psychology 2017, Chemnitz, Germany.

Dassen, F.C.M. (2017). Cognitive control interventions to combat globesity. Oral presentation given at the annual PhD-day of the Postgraduate School of Experimental Psychopathology, Utrecht, The Netherlands.

Dassen, F.C.M., Houben, K., Nederkoorn, C., \& Jansen, A. (2016). Werkgeheugentraining in spelformaat. E-health demonstration at the Najaarscongres van de Vereniging voor Gedragstherapie en Cognitieve Therapie, Veldhoven, The Netherlands.

Dassen, F.C.M. (2016). Cognitive control interventions to combat globesity. Update about this project presented at the Eatwell Workshop, Maastricht, The Netherlands

Dassen, F.C.M., Houben, K., Van Breukelen, G.J.P., \& Jansen, A. (2016). Cognitive control interventions to combat globesity: Testing the effectiveness of a Working Memorytraining. Oral presentation at Annual Research Day FPN, Maastricht, the Netherlands.

Dassen, F.C.M., Houben, K., Nederkoorn, C., \& Jansen, A. (2016). And switch! A pilot of a one-session set-shifting training in order to improve cognitive control. Poster presented at the annual meeting of the British Feeding and Drinking Group, London, England.

Dassen, F.C.M., Houben, K., Jansen, A. (2016). Visualizing your future: Exploring the effects of a future-self manipulation on eating behavior. Poster presented at the Association for Researchers in Psychology and Health, Maastricht, The Netherlands.

Dassen, F.C.M., Houben, K., Nederkoorn, C., \& Jansen, A. (2015) Eetgedrag en tijdsoriëntatie: De eerste stappen naar een interventie gericht op toekomstdenken. Oral presentation at the Najaarscongres van de Vereniging voor Gedragstherapie en Cognitieve Therapie, Veldhoven, The Netherlands.

Dassen, F.C.M., Van den Akker, K., Schyns, G. Lecture for 'Kidzcollege' (2015): Oral presentation and demonstration on general principles of psychology for primary school children, Maastricht, The Netherlands.

Dassen, F.C.M., Houben, K., Nederkoorn, C., \& Jansen, A. (2015). Focus on the future: Episodic future thinking in order to reduce discount rate and snacking. Poster presented at the annual meeting of the British Feeding and Drinking Group, Wageningen, The Netherlands. 
Dassen, F.C.M., Houben, K., \& Jansen, A. (2015). Time orientation and healthy eating: Unhealthy eaters consider immediate consequences, while healthy eaters focus on future health. Poster presented at the International Convention of Psychological Science, Amsterdam, The Netherlands.

Dassen, F.C.M., Van den Akker, K. (2014). Lecture for 'Kidzcollege'. Oral presentation and demonstration on general principles of psychology for primary school children, Maastricht, The Netherlands. 


\section{REFERENCES}

Adams, J. (2012). Consideration of immediate and future consequences, smoking status, and body mass index. Health Psychology, 31(2), 260-263. http://dx.doi.org/10.1037/a0025790

Adams, J., \& Nettle, D. (2009). Time perspective, personality and smoking, body mass, and physical activity: An empirical study. British journal of health psychology, 14(1), 83-105. http://dx.doi. org/10.1348/135910708X299664

Ainslie, G. (1975). Specious reward: a behavioral theory of impulsiveness and impulse control. Psychological bulletin, 82(4), 463-496. http://dx.doi.org/10.1037/h0076860

Ajzen, I. (1991). The theory of planned behavior. Organizational behavior and human decision processes, 50(2), 179-211. https://doi.org/10.1016/0749-5978(91)90020-T

Allan, J. L., Johnston, M., \& Campbell, N. (2010). Unintentional eating. What determines goalincongruent chocolate consumption? Appetite, 54(2), 422-425. https://doi.org/10.1016/j. appet.2010.01.009

Allan, J. L., Johnston, M., \& Campbell, N. (2011). Missed by an inch or a mile? Predicting the size of intention-behaviour gap from measures of executive control. Psychology \& health, 26(6), 635650. https://doi.org/10.1080/08870441003681307

Allom, V. (2014). The Role and Trainability of Executive Function in the Context of Healthy Eating. (Doctoral dissertation, Sydney Digital Theses). Retrieved from http://hdl.handle. net/2123/11639

Allom, V., Mullan, B., \& Hagger, M. (2016). Does inhibitory control training improve health behaviour? A meta-analysis. Health Psychology Review, 10(2), 168-186. https://doi.org/10.10 80/17437199.2015.1051078

Allom, V., Panetta, G., Mullan, B., \& Hagger, M. S. (2016). Self-report and behavioural approaches to the measurement of self-control: Are we assessing the same construct? Personality and Individual Differences, 90, 137-142. https://doi.org/10.1016/j.paid.2015.10.051

Alosco, M. L., Galioto, R., Spitznagel, M. B., Strain, G., Devlin, M., Cohen, R., . . Gunstad, J. (2014). Cognitive function after bariatric surgery: evidence for improvement 3 years after surgery. The American Journal of Surgery, 207(6), 870-876. http://doi.org/10.1016/j.amjsurg.2013.05.018

Alvarez, J. A., \& Emory, E. (2006). Executive function and the frontal lobes: a meta-analytic review. Neuropsychology review, 16(1), 17-42. https://doi.org/10.1007/s11065-006-9002-x

Appelhans, B. M., French, S. A., Pagoto, S. L., \& Sherwood, N. E. (2016). Managing temptation in obesity treatment: a neurobehavioral model of intervention strategies. Appetite, 96, 268-279. https://doi.org/10.1016/j.appet.2015.09.035

Appelhans, B. M., Waring, M. E., Schneider, K. L., Pagoto, S. L., DeBiasse, M. A., Whited, M. C., \& Lynch, E. B. (2012). Delay discounting and intake of ready-to-eat and away-from-home foods in overweight and obese women. Appetite, 59(2), 576-584. https://doi.org/10.1016/j. appet.2012.07.009

Appelhans, B. M., Woolf, K., Pagoto, S. L., Schneider, K. L., Whited, M. C., \& Liebman, R. (2011). Inhibiting food reward: delay discounting, food reward sensitivity, and palatable food intake in overweight and obese women. Obesity, 19(11), 2175-2182. http://dx.doi.org/10.1038/ oby.2011.57

Ariza, M., Garolera, M., Jurado, M. A., Garcia-Garcia, I., Hernan, I., Sanchez-Garre, C., . . Pueyo, R. (2012). Dopamine genes (DRD2/ANKK1-TaqA1 and DRD4-7R) and executive function: their interaction with obesity. PloS one, 7(7), e41482. https://doi.org/10.1371/journal.pone.0041482 
Arnocky, S., Milfont, T. L., \& Nicol, J. R. (2013). Time Perspective and Sustainable Behavior: Evidence for the Distinction Between Consideration of Immediate and Future Consequences. Environment and Behavior, 46, 556-582. https://doi.org/10.1177/0013916512474987

Atance, C. M., \& O'Neill, D. K. (2001). Episodic future thinking. Trends in cognitive sciences, 5(12), 533-539. https://doi.org/10.1016/S1364-6613(00)01804-0

Baddeley, A. (1992). Working memory. Science, 255(5044), 556-559. https://doi.org/10.1126/ science. 1736359

Baddeley, A. (2000). The episodic buffer: a new component of working memory? Trends in cognitive sciences, 4(11), 417-423. https://doi.org/10.1016/S1364-6613(00)01538-2

Baer, R. A., Smith, G. T., Hopkins, J., Krietemeyer, J., \& Toney, L. (2006). Using self-report assessment methods to explore facets of mindfulness. Assessment, 13(1), 27-45. https://doi. org/10.1177/1073191105283504

Baker, C. W., Little, T. D., \& Brownell, K. D. (2003). Predicting adolescent eating and activity behaviors: the role of social norms and personal agency. Health Psychology, 22(2), 189-198. doi: 10.1037/0278-6133.22.2.189

Baker, F., Johnson, M. W., \& Bickel, W. K. (2003). Delay discounting in current and never-before cigarette smokers: similarities and differences across commodity, sign, and magnitude. Journal of abnormal psychology, 112(3), 382-392. http://dx.doi.org/10.1037/0021-843X.112.3.382

Bar, M. (2010). Wait for the second marshmallow? Future-oriented thinking and delayed reward discounting in the brain. Neuron, 66(1), 4-5. https://doi.org/10.1016/j.neuron.2010.04.001

Barkley, R. A., Edwards, G., Laneri, M., Fletcher, K., \& Metevia, L. (2001). Executive functioning, temporal discounting, and sense of time in adolescents with attention deficit hyperactivity disorder (ADHD) and oppositional defiant disorder (ODD). Journal of abnormal child psychology, 29(6), 541-556. https://doi.org/10.1023/A:1012233310098

Barlow, P., Reeves, A., McKee, M., Galea, G., \& Stuckler, D. (2016). Unhealthy diets, obesity and time discounting: a systematic literature review and network analysis. obesity reviews, 17(9), 810819. http://dx.doi.org/10.1111/obr.12431

Barrett, L. F., Tugade, M. M., \& Engle, R. W. (2004). Individual Differences in Working Memory Capacity and Dual-Process Theories of the Mind. Psychological Bulletin, 130(4), 553-573. http://doi.org/10.1037/0033-2909.130.4.553

Barsics, C., Van der Linden, M., \& D’Argembeau, A. (2016). Frequency, characteristics, and perceived functions of emotional future thinking in daily life. The Quarterly Journal of Experimental Psychology, 69(2), 217-233. https://doi.org/10.1080/17470218.2015.1051560

Bartholdy, S., Dalton, B., O'Daly, O. G., Campbell, I. C., \& Schmidt, U. (2016). A systematic review of the relationship between eating, weight and inhibitory control using the stop signal task. Neuroscience \& Biobehavioral Reviews, 64(Supplement C), 35-62. doi: https://doi.org/10.1016/j. neubiorev.2016.02.010

Baumeister, R. F., Vohs, K. D., \&Tice, D. M. (2007). The strength model of self-control. Current directions in psychological science, 16(6), 351-355. https://doi.org/10.1111/j.1467-8721.2007.00534.x

Bayuk, J. B., Janiszewski, C., \& Leboeuf, R. A. (2010). Letting good opportunities pass us by: Examining the role of mind-set during goal pursuit. Journal of Consumer Research, 37(4), 570583. https://doi.org/10.1086/654892

Bechara, A. (2005). Decision making, impulse control and loss of willpower to resist drugs: a neurocognitive perspective. Nature neuroscience, 8(11), 1458-1463. doi:10.1038/nn1584 
Bechara, A., \& Martin, E. M. (2004). Impaired decision making related to working memory deficits in individuals with substance addictions. Neuropsychology, 18(1), 152-162. http://dx.doi. org/10.1037/0894-4105.18.1.152

Beck, J. S. (2008). Beck's dieetoplossing: Train je brein, denk slank. (M. van der Horst, Trans.). Amsterdam: Uitgeverij Nieuwezijds. (Original work published in 2007).

Benoit, R. G., Gilbert, S. J., \& Burgess, P. W. (2011). A neural mechanism mediating the impact of episodic prospection on farsighted decisions. The Journal of Neuroscience, 31(18), 6771-6779. https://doi.org/10.1523/jneurosci.6559-10.2011

Berg, E. A. (1948). A simple objective technique for measuring flexibility in thinking. The Journal of general psychology, 39(1), 15-22. https://doi.org/10.1080/00221309.1948.9918159

Best, J. R., Theim, K. R., Gredysa, D. M., Stein, R. I., Welch, R. R., Saelens, B. E., ... Wilfley, D. E. (2012). Behavioral Economic Predictors of Overweight Children's Weight Loss. Journal of Consulting and Clinical Psychology, 80(6), 1086-1096. http://doi.org/10.1037/a0029827

Best, M., Lawrence, N. S., Logan, G. D., McLaren, I. P., \& Verbruggen, F. (2016). Should I stop or should I go? The role of associations and expectancies. Journal of experimental psychology: human perception and performance, 42(1), 115-137. http://dx.doi.org/10.1037/xhp0000116

Bickel, W. K., Jarmolowicz, D. P., Mueller, E.T., Gatchalian, K. M., \& McClure, S. M. (2012). Are executive function and impulsivity antipodes? A conceptual reconstruction with special reference to addiction. Psychopharmacology, 221(3), 361-387. https://doi.org/10.1007/s00213-012-2689-x

Bickel, W. K., \& Marsch, L. A. (2001). Toward a behavioral economic understanding of drug dependence: delay discounting processes. Addiction, 96(1), 73-86. http://dx.doi.org/10.1046/ j.1360-0443.2001.961736.x

Bickel, W. K., Miller, M. L., Yi, R., Kowal, B. P., Lindquist, D. M., \& Pitcock, J. A. (2007). Behavioral and neuroeconomics of drug addiction: competing neural systems and temporal discounting processes. Drug and alcohol dependence, 90, S85-S91. https://doi.org/10.1016/j. drugalcdep.2006.09.016

Bickel, W. K., \& Yi, R. (2008). Temporal discounting as a measure of executive function: Insights from the competing neuro-behavioral decision system hypothesis of addiction. In Neuroeconomics (289-309). Bingley: Emerald Group Publishing Limited. doi:10.1016/S0731-2199(08)20012-9

Bickel, W. K., Yi, R., Landes, R. D., Hill, P. F., \& Baxter, C. (2011). Remember the future: working memory training decreases delay discounting among stimulant addicts. Biological psychiatry, 69(3), 260-265. https://doi.org/10.1016/j.biopsych.2010.08.017

Blackburn, G. (1995). Effect of degree of weight loss on health benefits. Obesity, 3(S2), 211s- 216s. http://dx.doi.org/10.1002/j.1550-8528.1995.tb00466.x

Boeka, A. G., \& Lokken, K. L. (2008). Neuropsychological performance of a clinical sample of extremely obese individuals. Archives of Clinical Neuropsychology, 23(4), 467-474. https://doi. org/10.1016/j.acn.2008.03.003

Boendermaker, W. J., Prins, P. J., \& Wiers, R. W. (2015). Cognitive Bias Modification for adolescents with substance use problems-Can serious games help? Journal of behavior therapy and experimental psychiatry, 49, 13-20. https://doi.org/10.1016/j.jbtep.2015.03.008

Boh, B., Lemmens, L. H., Jansen, A., Nederkoorn, C., Kerkhofs, V., Spanakis, G., .. Roefs, A. (2016). An Ecological Momentary Intervention for weight loss and healthy eating via smartphone and Internet: study protocol for a randomised controlled trial. Trials, 17(1), 154. https://doi. org/10.1186/s13063-016-1280-x 
Boselie, J. J., Vancleef, L. M., \& Peters, M. L. (2016). The effects of experimental pain and induced optimism on working memory task performance. Scandinavian Journal of Pain, 12, 25-32. https://doi.org/10.1016/j.sjpain.2016.03.001

Boswell, R. G., \& Kober, H. (2016). Food cue reactivity and craving predict eating and weight gain: a meta-analytic review. Obesity Reviews, 17(2), 159-177. http://dx.doi.org/10.1111/obr.12354

Botvinick, M., \& Braver, T. (2015). Motivation and cognitive control: from behavior to neural mechanism. Annual review of psychology, 66, 83-113. https://doi.org/10.1146/annurevpsych-010814-015044

Boutelle, K. N., \& Kirschenbaum, D. S. (1998). Further support for consistent self-monitoring as a vital component of successful weight control. Obesity Research, 6(3), 219-224.

Bowie, C. R., \& Harvey, P. D. (2006). Administration and interpretation of the Trail Making Test. Nature protocols, 1(5), 2277-2281. doi:10.1038/nprot.2006.390

Boyer, P. (2008). Evolutionary economics of mental time travel? Trends in cognitive sciences, 12(6), 219-224. doi: http://dx.doi.org/10.1016/j.tics.2008.03.003

Brewin, C. R., \& Smart, L. (2005). Working memory capacity and suppression of intrusive thoughts. Journal of behavior therapy and experimental psychiatry, 36(1), 61-68. https://doi.org/10.1016/j. jbtep.2004.11.006

Budría, S., Lacomba, J. A., Lagos, F. M., \& Swedberg, P. (2012). When obese people are more patient than non-obese people. A study of post-surgery individuals in a weight loss association. Revista Internacional de Sociología, 70(1), 83-98. http://hdl.handle.net/10481/38616

Butryn, M. L., Webb, V., \& Wadden, T. A. (2011). Behavioral treatment of obesity. The Psychiatric clinics of North America, 34(4), 841-859. https://doi.org/10.1016/j.psc.2011.08.006

Chaput, J. P., Klingenberg, L., Astrup, A., \& Sjödin, A. M. (2011). Modern sedentary activities promote overconsumption of food in our current obesogenic environment. Obesity Reviews, 12(5), e12-e20. http://dx.doi.org/10.1111/j.1467-789X.2010.00772.x

Cheng, Y. Y., Shein, P. P., \& Chiou, W. B. (2012). Escaping the impulse to immediate gratification: The prospect concept promotes a future-oriented mindset, prompting an inclination towards delayed gratification. British Journal of Psychology, 103(1), 129-141. http://dx.doi.org/10.1111/ j.2044-8295.2011.02067.x

Chung, S. H., \& Herrnstein, R. J. (1967). Choice and delay of reinforcement. Journal of the experimental analysis of behavior, 10(1), 67-74. http://dx.doi.org/10.1901/jeab.1967.10-67

Cohen, J., Cohen, P., West, S. G., \& Aiken, L. S. (2013). Applied multiple regression/correlation analysis for the behavioral sciences. New Jersey: Routledge.

Cserjési, R., Molnár, D., Luminet, O., \& Lénárd, L. (2007). Is there any relationship between obesity and mental flexibility in children? Appetite, 49(3), 675-678. https://doi.org/10.1016/j. appet.2007.04.001

D'Argembeau, A., Renaud, O., \& Van der Linden, M. (2011). Frequency, characteristics and functions of future-oriented thoughts in daily life. Applied Cognitive Psychology, 25(1), 96-103. http:// dx.doi.org/10.1002/acp.1647

Dahlgren, C. L., \& Rø, Ø. (2014). A systematic review of cognitive remediation therapy for anorexia nervosa-development, current state and implications for future research and clinical practice. Journal of eating disorders, 2(1), 26. https://doi.org/10.1186/s40337-014-0026-y 
Daniel, T. O., Said, M., Stanton, C. M., \& Epstein, L. H. (2015). Episodic future thinking reduces delay discounting and energy intake in children. Eating behaviors, 18, 20-24. https://doi. org/10.1016/j.eatbeh.2015.03.006

Daniel, T. O., Stanton, C. M., \& Epstein, L. H. (2013a). The future is now: Comparing the effect of episodic future thinking on impulsivity in lean and obese individuals. Appetite, 71, 120-125. https://doi.org/10.1016/j.appet.2013.07.010

Daniel, T. O., Stanton, C. M., \& Epstein, L. H. (2013b). The future is now: Reducing impulsivity and energy intake using episodic future thinking. Psychological science, 2339-2342. https://doi. org/10.1177/0956797613488780

Dansinger, M. L., Tatsioni, A., Wong, J. B., Chung, M., \& Balk, E. M. (2007). Meta-analysis: the effect of dietary counseling for weight loss. Annals of internal medicine, 147(1), 41-50. doi: 10.7326/0003-4819-147-1-200707030-00007

Dassen, F. C. M., Houben, K., \& Jansen, A. (2015). Time orientation and eating behavior: Unhealthy eaters consider immediate consequences, while healthy eaters focus on future health. Appetite, 91, 13-19. https://doi.org/10.1016/j.appet.2015.03.020

Dassen, F. C. M., Jansen, A., Nederkoorn, C., Houben, K. (2016). Focus on the future: Episodic future thinking reduces discount rate and snacking. Appetite, 96, 327-332. https://doi.org/10.1016/j. appet.2015.09.032

Dassen, F. C. M., Houben, K., Van Breukelen, G. J., \& Jansen, A. (2018). Gamified working memory training in overweight individuals reduces food intake but not body weight. Appetite, 124, 89-98. https://doi.org/10.1016/j.appet.2017.05.009

Daugherty, J. R., \& Brase, G. L. (2010). Taking time to be healthy: Predicting health behaviors with delay discounting and time perspective. Personality and Individual Differences, 48(2), 202-207. https://doi.org/10.1016/j.paid.2009.10.007

Davis, C., Patte, K., Curtis, C., \& Reid, C. (2010). Immediate pleasures and future consequences. A neuropsychological study of binge eating and obesity. Appetite, 54(1), 208-213. https://doi. org/10.1016/j.appet.2009.11.002

De Graaf, C. (2006). Effects of snacks on energy intake: an evolutionary perspective. Appetite, 47(1), 18-23. https://doi.org/10.1016/j.appet.2006.02.007

De Ridder, D., Adriaanse, M., Evers, C., \& Verhoeven, A. (2014). Who diets? Most people and especially when they worry about food. Appetite, 80, 103-108. https://doi.org/10.1016/j. appet.2014.05.011

Deitel, M., \& Greenstein, R. J. (2003). Recommendations for reporting weight loss. Obesity surgery, 13(2), 159-160. https://doi.org/10.1381/096089203764467117

Deterding, S., Dixon, D., Khaled, R., \& Nacke, L. (2011). From game design elements to gamefulness: defining gamification. Paper presented at the Proceedings of the 15th international academic MindTrek conference: Envisioning future media environments.

DeSmet, A., Van Ryckeghem, D., Compernolle, S., Baranowski, T., Thompson, D., Crombez, G., ... Van Cleemput, K. (2014). A meta-analysis of serious digital games for healthy lifestyle promotion. Preventive medicine, 69, 95-107. https://doi.org/10.1016/j.ypmed.2014.08.026

Diamond, A. (2013). Executive functions. Annual review of psychology, 64, 135-168. https://doi. org/10.1146/annurev-psych-113011-143750

Diamond, A., \& Lee, K. (2011). Interventions shown to aid executive function development in children 4 to 12 years old. Science, 333(6045), 959-964. doi: 10.1126/science.1204529 
Diamond, A., \& Ling, D. S. (2016). Conclusions about interventions, programs, and approaches for improving executive functions that appear justified and those that, despite much hype, do not. Developmental cognitive neuroscience, 18, 34-48. https://doi.org/10.1016/j. dcn.2015.11.005

Dixon, M. R., Marley, J., \& Jacobs, E. A. (2003). Delay discounting by pathological gamblers. Journal of applied behavior analysis, 36(4), 449-458. http://dx.doi.org/10.1901/jaba.2003.36-449

Dohle, S., Diel, K., \& Hofmann, W. (2018). Executive functions and the self-regulation of eating behavior: A review. Appetite, 124, 4-9. https://doi.org/10.1016/j.appet.2017.05.041

Douketis, J., Macie, C., Thabane, L., \& Williamson, D. (2005). Systematic review of long-term weight loss studies in obese adults: clinical significance and applicability to clinical practice. International Journal of Obesity, 29(10), 1153-1167. doi:10.1038/sj.ijo.0802982

Drewnowski, A., \& Greenwood, M. (1983). Cream and sugar: human preferences for high-fat foods. Physiology \& Behavior, 30(4), 629-633. https://doi.org/10.1016/0031-9384(83)90232-9

Duckworth, A. L., \& Kern, M. L. (2011). A meta-analysis of the convergent validity of self-control measures. Journal of Research in Personality, 45(3), 259-268. https://doi.org/10.1016/j. jrp.2011.02.004

Dunn, C., Hannan, P., Jeffery, R., Sherwood, N., Pronk, N., \& Boyle, R. (2006). The comparative and cumulative effects of a dietary restriction and exercise on weight loss. International Journal of Obesity, 30(1), 112-121. doi:10.1038/sj.ijo.0803046

Epstein, L. H., Jankowiak, N., Fletcher, K. D., Carr, K. A., Nederkoorn, C., Raynor, H. A., \& Finkelstein, E. (2014). Women who are motivated to eat and discount the future are more obese. Obesity, 22(6), 1394-1399. http://dx.doi.org/10.1002/oby.20661

Epstein, L. H., Salvy, S. J., Carr, K. A., Dearing, K. K., \& Bickel, W. K. (2010). Food reinforcement, delay discounting and obesity. Physiology \& Behavior, 100(5), 438-445. https://doi.org/10.1016/j. physbeh.2010.04.029

Epstein, L. H., Valoski, A., Wing, R. R., \& McCurley, J. (1994). Ten-year outcomes of behavioral familybased treatment for childhood obesity. Health Psychology, 13(5), 373-383.

Estle, S. J., Green, L., Myerson, J., \& Holt, D. D. (2007). Discounting of monetary and directly consumable rewards. Psychological science, 18(1), 58-63. https://doi.org/10.1111\%2Fj.14679280.2007.01849.x

Etnier, J. L., \& Chang, Y.-K. (2009). The effect of physical activity on executive function: a brief commentary on definitions, measurement issues, and the current state of the literature. Journal of Sport and Exercise Psychology, 31(4), 469-483. https://doi.org/10.1123/jsep.31.4.469

Fagundo, A. B., De la Torre, R., Jiménez-Murcia, S., Agüera, Z., Granero, R., Tárrega, S., . . Rodríguez, R. (2012). Executive functions profile in extreme eating/weight conditions: from anorexia nervosa to obesity. PloS one, 7(8), e43382. https://doi.org/10.1371/journal.pone.0043382

Fairburn, C. G., \& Beglin, S. (2008). Eating disorder examination questionnaire. In C.G. Fairburn (Ed.) Cognitive behaviour therapy and eating disorders (309-313). New York, NY: Guilford Press.

Feda, D. M., Roemmich, J. N., Roberts, A., \& Epstein, L. H. (2015). Food reinforcement and delay discounting in zBMI-discordant siblings. Appetite, 85, 185-189. https://doi.org/10.1016/j. appet.2014.11.023

Fergenbaum, J. H., Bruce, S., Lou, W., Hanley, A. J., Greenwood, C., \& Young, T. K. (2009). Obesity and lowered cognitive performance in a Canadian First Nations population. Obesity, 17(10), 19571963. http://dx.doi.org/10.1038/oby.2009.161 
Field, M., Santarcangelo, M., Sumnall, H., Goudie, A., \& Cole, J. (2006). Delay discounting and the behavioural economics of cigarette purchases in smokers: the effects of nicotine deprivation. Psychopharmacology, 186(2), 255-263.

Finucane, M. M., Stevens, G. A., Cowan, M. J., Danaei, G., Lin, J. K., Paciorek, C. J., . . Bahalim, A. N. (2011). National, regional, and global trends in body-mass index since 1980: systematic analysis of health examination surveys and epidemiological studies with 960 country-years and 9. 1 million participants. The Lancet, 377(9765), 557-567. https://doi.org/10.1016/S01406736(10)62037-5

Fitzpatrick, S., Gilbert, S., \& Serpell, L. (2013). Systematic review: are overweight and obese individuals impaired on behavioural tasks of executive functioning? Neuropsychology review, 23(2), 138-156. https://doi.org/10.1007/s11065-013-9224-7

Flegal, K. M., Carroll, M. D., Kit, B. K., \& Ogden, C. L. (2012). Prevalence of obesity and trends in the distribution of body mass index among US adults, 1999-2010. Jama, 307(5), 491-497. doi:10.1001/jama.2012.39

Frederick, S. (2006). Valuing future life and future lives: A framework for understanding discounting. Journal of Economic Psychology, 27(5), 667-680. https://doi.org/10.1016/j.joep.2006.05.007

Friedman, N. P., \& Miyake, A. (2017). Unity and diversity of executive functions: Individual differences as a window on cognitive structure. Cortex, 86, 186-204. https://doi.org/10.1016/j. cortex.2016.04.023

Friedman, N. P., Miyake, A., Young, S. E., DeFries, J. C., Corley, R. P., \& Hewitt, J. K. (2008). Individual differences in executive functions are almost entirely genetic in origin. Journal of Experimental Psychology: General, 137(2), 201-225. http://dx.doi.org/10.1037/0096-3445.137.2.201

Friese, M., Hofmann, W., \& Wänke, M. (2008). When impulses take over: Moderated predictive validity of explicit and implicit attitude measures in predicting food choice and consumption behaviour. British Journal of Social Psychology, 47(3), 397-419. http://dx.doi. org/10.1348/014466607X241540

Friese, M., Hofmann, W., \& Wiers, R. W. (2011). On taming horses and strengthening riders: Recent developments in research on interventions to improve self-control in health behaviors. Self and Identity, 10(3), 336-351. https://doi.org/10.1080/15298868.2010.536417

Galioto, R., Bond, D., Gunstad, J., Pera, V., Rathier, L., \& Tremont, G. (2016). Executive functions predict weight loss in a medically supervised weight loss programme. Obesity science \& practice, 2(4), 334-340. doi: 10.1002/osp4.70

Gawronski, B., \& Creighton, L. A. (2013). Dual-process theories. In D.E. Carlston (Ed.)The Oxford handbook of social cognition (282-312). New York, NY: Oxford University Press.

Gettens, K. M., \& Gorin, A. A. (2017). Executive function in weight loss and weight loss maintenance: a conceptual review and novel neuropsychological model of weight control. Journal of behavioral medicine, 40(5), 1-15. https://doi.org/10.1007/s10865-017-9831-5

Gick, M. (2014). An exploration of interactions between Conscientiousness and Consideration of Future Consequences on healthy eating. Personality and Individual Differences, 66, 181-187. https://doi.org/10.1016/j.paid.2014.03.020

Goldstein, D. J. (1992). Beneficial health effects of modest weight loss. International journal of obesity and related metabolic disorders: journal of the International Association for the Study of Obesity, 16(6), 397-415. 
Gonzales, M. M., Tarumi, T., Miles, S. C., Tanaka, H., Shah, F., \& Haley, A. P. (2010). Insulin sensitivity as a mediator of the relationship between $\mathrm{BMI}$ and working memory-related brain activation. Obesity, 18(11), 2131-2137. http://dx.doi.org/10.1038/oby.2010.183

Grant, D. A., \& Berg, E. (1948). A behavioral analysis of degree of reinforcement and ease of shifting to new responses in a Weigl-type card-sorting problem. Journal of experimental psychology, 38(4), 404. http://dx.doi.org/10.1037/h0059831

Green, C. S., \& Bavelier, D. (2008). Exercising your brain: a review of human brain plasticity and training-induced learning. Psychology and aging, 23, 692-701. doi: 10.1037/a0014345

Green, L., \& Myerson, J. (2013). How many impulsivities? A discounting perspective. Journal of the experimental analysis of behavior, 99(1), 3-13. http://dx.doi.org/10.1002/jeab.1

Greve, K. W., Stickle, T. R., Love, J. M., Bianchini, K. J., \& Stanford, M. S. (2005). Latent structure of the Wisconsin Card Sorting Test: a confirmatory factor analytic study. Archives of Clinical Neuropsychology, 20(3), 355-364. https://doi.org/10.1016/j.acn.2004.09.004

Guerrieri, R., Nederkoorn, C., \& Jansen, A. (2012). Disinhibition is easier learned than inhibition. The effects of (dis) inhibition training on food intake. Appetite, 59(1), 96-99. https://doi. org/10.1016/j.appet.2012.04.006

Guerrieri, R., Nederkoorn, C., Schrooten, M., Martijn, C., \& Jansen, A. (2009). Inducing impulsivity leads high and low restrained eaters into overeating, whereas current dieters stick to their diet. Appetite, 53(1), 93-100. https://doi.org/10.1016/j.appet.2009.05.013

Gunstad, J., Paul, R. H., Cohen, R. A., Tate, D. F., Spitznagel, M. B., \& Gordon, E. (2007). Elevated body mass index is associated with executive dysfunction in otherwise healthy adults. Comprehensive psychiatry, 48(1), 57-61. https://doi.org/10.1016/j.comppsych.2006.05.001

Halberstadt, J., Makkes, S., De Vet, E., Jansen, A., Nederkoorn, C., van der Baan-Slootweg, O. H., \& Seidell, J.C. (2013). The role of self-regulating abilities in long-term weight loss in severely obese children and adolescents undergoing intensive combined lifestyle interventions (HELIOS); rationale, design and methods. BMC pediatrics, 13(1), 41. https://doi.org/10.1186/1471-243113-41

Hall, P. A. (2012). Executive control resources and frequency of fatty food consumption: findings from an age-stratified community sample. Health Psychology, 31(2), 235-241. doi: 10.1037/ a0025407

Hall, P. A., \& Fong, G. T. (2003). The effects of a brief time perspective intervention for increasing physical activity among young adults. Psychology and Health, 18(6), 685-706. https://doi. org/10.1080/0887044031000110447

Hall, P. A., \& Fong, G. T. (2007). Temporal self-regulation theory: A model for individual health behavior. Health Psychology Review, 1 (1), 6-52. https://doi.org/10.1080/17437190701492437

Hall, P. A., Fong, G. T., \& Cheng, A. Y. (2012). Time perspective and weight management behaviors in newly diagnosed Type 2 diabetes: a mediational analysis. Journal of behavioral medicine, 35(6), 569-580. https://doi.org/10.1007/s10865-011-9389-6

Hall, P. A., \& Marteau, T. M. (2014). Executive function in the context of chronic disease prevention: theory, research and practice. Preventive medicine, 68, 44-50. https://doi.org/10.1016/j. ypmed.2014.07.008

Harrison, T. L., Shipstead, Z., Hicks, K. L., Hambrick, D. Z., Redick, T. S., \& Engle, R. W. (2013). Working memory training may increase working memory capacity but not fluid intelligence. Psychological science, 24(12), 2409-2419. https://doi.org/10.1177/0956797613492984 
Hege, M. A., Stingl, K., Ketterer, C., Häring, H.-U., Heni, M., Fritsche, A., \& PreißI, H. (2013). Working memory-related brain activity is associated with outcome of lifestyle intervention. Obesity, 21(12), 2488-2494. http://dx.doi.org/10.1002/oby.20442

Hendrick, O. M., Luo, X., Zhang, S., \& Li, C. S. R. (2012). Saliency processing and obesity: a preliminary imaging study of the stop signal task. Obesity, 20(9), 1796-1802. doi: 10.1038/oby.2011.180

Herman, C.P., Polivy, J.P. (1980). Restrained eating. In A.J. Stunkard (Ed.), Obesity (208225), Philadelphia, PA: Saunders.

Hill, J. O., Melanson, E. L., \& Wyatt, H. T. (2000). Dietary fat intake and regulation of energy balance: implications for obesity. The Journal of nutrition, 130(2), 284S-288S.

Hill, J. O., \& Peters, J. C. (1998). Environmental contributions to the obesity epidemic. Science, 280(5368), 1371-1374. doi: 10.1126/science.280.5368.1371

Hofmann, W., Friese, M., \& Roefs, A. (2009). Three ways to resist temptation: The independent contributions of executive attention, inhibitory control, and affect regulation to the impulse control of eating behavior. Journal of Experimental Social Psychology, 45(2), 431-435. https:// doi.org/10.1016/j.jesp.2008.09.013

Hofmann, W., Friese, M., \&Strack, F. (2009). Impulse and self-control from a dual-systems perspective. Perspectives on Psychological Science, 4(2), 162-176. https://doi.org/10.1111\%2Fj.17456924.2009.01116.x

Hofmann, W., Gschwendner, T., Friese, M., Wiers, R. W., \& Schmitt, M. (2008). Working memory capacity and self-regulatory behavior: toward an individual differences perspective on behavior determination by automatic versus controlled processes. Journal of personality and social psychology, 95(4), 962-977. http://dx.doi.org/10.1037/a0012705

Hofmann, W., Schmeichel, B. J., \& Baddeley, A. D. (2012). Executive functions and self-regulation. Trends in cognitive sciences, 16(3), 174-180. https://doi.org/10.1016/j.tics.2012.01.006

Hofmann, W., \& Van Dillen, L. (2012). Desire The New Hot Spot in Self-Control Research. Current directions in psychological science, 21(5), 317-322. https://doi.org/10.1177/0963721412453587

Holmes, J., Gathercole, S. E., \& Dunning, D. L. (2009). Adaptive training leads to sustained enhancement of poor working memory in children. Developmental science, 12(4), F9-F15. doi: 10.1111/j.1467-7687.2009.00848.x

Houben, K., Dassen, F.C.M., \& Jansen, A. (2016). Taking control: Working memory training in overweight individuals increases self-regulation of food intake. Appetite, 105, 567-574. https://doi.org/10.1016/j.appet.2016.06.029

Houben, K., Havermans, R. C., Nederkoorn, C., \& Jansen, A. (2012). Beer à No-Go: Learning to stop responding to alcohol cues reduces alcohol intake via reduced affective associations rather than increased response inhibition. Addiction, 107(7), 1280-1287. doi: 10.1111/j.13600443.2012.03827.x

Houben, K., \& Jansen, A. (2015). Chocolate equals stop. Chocolate-specific inhibition training reduces chocolate intake and go associations with chocolate. Appetite, 87, 318-323. https:// doi.org/10.1016/j.appet.2015.01.005

Houben, K., Nederkoorn, C., \& Higgs, S. (2018). An introduction to the special issue on 'Executive function training \& eating behaviour', Appetite, 124, 1-3. https://doi.org/10.1016/j. appet.2017.09.021 
Houben, K., Nederkoorn, C., \& Jansen, A. (2014). Eating on impulse: The relation between overweight and food-specific inhibitory control. Obesity, 22(5), 968-975. doi: 10.1002/ oby. 20670

Houben, K., \&Wiers, R. W. (2009). Response inhibition moderates the relationship between implicit associations and drinking behavior. Alcoholism: Clinical and Experimental Research, 33(4), 626633. http://dx.doi.org/10.1111/j.1530-0277.2008.00877.x

Houben, K., Wiers, R. W., \& Jansen, A. (2011). Getting a grip on drinking behavior training working memory to reduce alcohol abuse. Psychological science, 22, 968-975. https://doi. org/10.1177/0956797611412392

Ikeda, J., Amy, N. K., Ernsberger, P., Gaesser, G. A., Berg, F. M., Clark, C. A., . . Peters, P. (2005). The national weight control registry: a critique. Journal of nutrition education and behavior, 37(4), 203-205. https://doi.org/10.1016/S1499-4046(06)60247-9

Jaeggi, S. M., Buschkuehl, M., Jonides, J., \& Shah, P. (2011). Short-and long-term benefits of cognitive training. Proceedings of the National Academy of Sciences, 108(25), 10081-10086. https://doi.org/10.1073/pnas.1103228108

Jaeggi, S. M., Buschkuehl, M., Perrig, W. J., \& Meier, B. (2010). The concurrent validity of the N-back task as a working memory measure. Memory, 18(4), 394-412. https://doi. org/10.1080/09658211003702171

Jansen, A., Houben, K., \& Roefs, A. (2015). A cognitive profile of obesity and its translation into new interventions. Frontiers in psychology, 6, 1807. https://doi.org/10.3389/fpsyg.2015.01807

Jansen, A., Nederkoorn, C., Roefs, A., Martijn, C., Havermans, R., \& Mulkens, S. (2009). Waarom obesitas in de GGZ behandeld moet worden. GZ-psychologie, 1(2), 38-44.

Jarmolowicz, D. P., Cherry, J. B. C., Reed, D. D., Bruce, J. M., Crespi, J. M., Lusk, J. L., \& Bruce, A. S. (2014). Robust relation between temporal discounting rates and body mass. Appetite, 78, 6367. https://doi.org/10.1016/j.appet.2014.02.013

Jimura, K., Myerson, J., Hilgard, J., Keighley, J., Braver, T. S., \& Green, L. (2011). Domain independence and stability in young and older adults' discounting of delayed rewards. Behavioural processes, 87(3), 253-259. https://doi.org/10.1016/j.beproc.2011.04.006

Johnson, M. W., \& Bickel, W. K. (2002). Within-subject comparison of real and hypothetical money rewards in delay discounting. Journal of the experimental analysis of behavior, 77(2), 129-146. http://dx.doi.org/10.1901/jeab.2002.77-129

Johnston, B. C., Kanters, S., Bandayrel, K., Wu, P., Naji, F., Siemieniuk, R. A., . . Guyatt, G. (2014). Comparison of weight loss among named diet programs in overweight and obese adults: a meta-analysis. Jama, 312(9), 923-933. doi:10.1001/jama.2014.10397

Joireman, J., Balliet, D., Sprott, D., Spangenberg, E., \& Schultz, J. (2008). Consideration of future consequences, ego-depletion, and self-control: Support for distinguishing between CFCImmediate and CFC-Future sub-scales. Personality and Individual Differences, 45(1), 15-21. https://doi.org/10.1016/j.paid.2008.02.011

Joireman, J., Shaffer, M. J., Balliet, D., \& Strathman, A. (2012). Promotion orientation explains why future-oriented people exercise and eat healthy: Evidence from the two-factor Consideration of Future Consequences-14 scale. Personality and Social Psychology Bulletin, 38(10), 1272 1287. https://doi.org/10.1177/0146167212449362 
Jones, A., Di Lemma, L. C., Robinson, E., Christiansen, P., Nolan, S., Tudur-Smith, C., \& Field, M. (2016). Inhibitory control training for appetitive behaviour change: A meta-analytic investigation of mechanisms of action and moderators of effectiveness. Appetite, 97, 16-28. https://doi. org/10.1016/j.appet.2015.11.013

Jones, A., Hardman, C. A., Lawrence, N., \& Field, M. (2018). Cognitive training as a potential treatment for overweight and obesity: A critical review of the evidence. Appetite, 124, 50-67. https://doi.org/10.1016/j.appet.2017.05.032

Juarascio, A. S., Manasse, S. M., Espel, H. M., Kerrigan, S. G., \& Forman, E. M. (2015). Could training executive function improve treatment outcomes for eating disorders? Appetite, 90, 187-193. https://doi.org/10.1016/j.appet.2015.03.013

Jurado, M. B., \& Rosselli, M. (2007). The elusive nature of executive functions: a review of our current understanding. Neuropsychology review, 17(3), 213-233. https://doi.org/10.1007/ s11065-007-9040-z

Kanoski, S. E., \& Davidson, T. L. (2011). Western diet consumption and cognitive impairment: links to hippocampal dysfunction and obesity. Physiology \& Behavior, 103(1), 59-68. https://doi. org/10.1016/j.physbeh.2010.12.003

Kaplan, B. A., Lemley, S. M., Reed, D. D., \& Jarmolowicz, D. P. (2014). 21- and 27-Item Monetary Choice Questionnaire Automated Scorers [software]. Center for Applied Neuroeconomics, University of Kansas. doi: 10.1007/s40614-016-0070-9

Karbach, J., \& Kray, J. (2009). How useful is executive control training? Age differences in near and far transfer of task-switching training. Developmental science, 12(6), 978-990. http://dx.doi. org/10.1111/j.1467-7687.2009.00846.x

Karbach, J., \& Verhaeghen, P. (2014). Making working memory work: a meta-analysis of executivecontrol and working memory training in older adults. Psychological science, 25(11), 20272037. doi: $10.1177 / 0956797614548725$

Kavanagh, D. J., Andrade, J., \& May, J. (2005). Imaginary relish and exquisite torture: the elaborated intrusion theory of desire. Psychological review, 112(2), 446-467. doi: 10.1037/0033295X.112.2.446

Kelly, S. M., \& Updegraff, J. A. (2017). Substituting activities mediates the effect of cognitive flexibility on physical activity: A daily diary study. Journal of behavioral medicine, 1-6. https:// doi.org/10.1007/s10865-017-9839-x

Kemps, E., \& Tiggemann, M. (2010). A cognitive experimental approach to understanding and reducing food cravings. Current directions in psychological science, 19(2), 86-90. https://doi. org/10.1177/0963721410364494

Kirby, K. N. (2000). Instructions for inferring discount rates from choices between immediate and delayed rewards. Unpublished manuscript. Williams College, Williamstown, MA.

Kirby, K. N. (2009). One-year temporal stability of delay-discount rates. Psychonomic Bulletin \& Review, 16(3), 457-462. https://doi.org/10.3758/PBR.16.3.457

Kirby, K. N., \& Maraković, N. N. (1996). Delay-discounting probabilistic rewards: Rates decrease as amounts increase. Psychonomic Bulletin \& Review, 3(1), 100-104. https://doi.org/10.3758/ BF03210748

Kirby, K. N., Petry, N. M., \& Bickel, W. K. (1999). Heroin addicts have higher discount rates for delayed rewards than non-drug-using controls. Journal of Experimental Psychology: General, 128(1), 78-87. doi: 10.1037/0096-3445.128.1.78 
Kirchner, W. K. (1958). Age differences in short-term retention of rapidly changing information. Journal of experimental psychology, 55(4), 352-358. http://psycnet.apa.org/doi/10.1037/ h0043688

Kishinevsky, F. I., Cox, J. E., Murdaugh, D. L., Stoeckel, L. E., Cook, E. W., \& Weller, R. E. (2012). fMRI reactivity on a delay discounting task predicts weight gain in obese women. Appetite, 58(2), 582-592. https://doi.org/10.1016/j.appet.2011.11.029

Klingberg, T. (2010). Training and plasticity of working memory. Trends in cognitive sciences, 14(7), 317-324. https://doi.org/10.1016/j.tics.2010.05.002

Klingberg, T., Fernell, E., Olesen, P. J., Johnson, M., Gustafsson, P., Dahlström, K., . . W Westerberg, H. (2005). Computerized training of working memory in children with ADHD-a randomized, controlled trial. Journal of the American Academy of Child \& Adolescent Psychiatry, 44(2), 177186. https://doi.org/10.1097/00004583-200502000-00010

Klingberg, T., Forssberg, H., \& Westerberg, H. (2002). Training of working memory in children with ADHD. Journal of clinical and experimental neuropsychology, 24(6), 781-791. doi: 10.1076/ jcen.24.6.781.8395

Kober, H., Kross, E. F., Mischel, W., Hart, C. L., \& Ochsner, K. N. (2010). Regulation of craving by cognitive strategies in cigarette smokers. Drug and alcohol dependence, 106(1), 52-55. https:// doi.org/10.1016/j.drugalcdep.2009.07.017

Koffarnus, M. N., Jarmolowicz, D. P., Mueller, E. T., \& Bickel, W. K. (2013). Changing delay discounting in the light of the competing neurobehavioral decision systems theory: a review. Journal of the experimental analysis of behavior, 99(1), 32-57. doi: 10.1002/jeab.2

Kovač, V. B., \& Rise, J. (2007). The relation between past behavior, intention, planning, and quitting smoking: The moderating effect of future orientation. Journal of Applied Biobehavioral Research, 12(2), 82-100. doi: 10.1111/j.1751-9861.2007.00015.x

Kuijer, R. G., \& Boyce, J. A. (2012). Emotional eating and its effect on eating behaviour after a natural disaster. Appetite, 58(3), 936-939. https://doi.org/10.1016/j.appet.2012.02.046

Kuijer, R. G., \& Boyce, J. A. (2014). Chocolate cake. Guilt or celebration? Associations with healthy eating attitudes, perceived behavioural control, intentions and weight-loss. Appetite, 74, 4854.

Kulendran, M., Vlaev, I., Sugden, C., King, D., Ashrafian, H., Gately, P., \& Darzi, A. (2014). Neuropsychological assessment as a predictor of weight loss in obese adolescents. International Journal of Obesity, 38(4), 507-512. doi:10.1038/ijo.2013.198

Lagorio, C. H., \& Madden, G. J. (2005). Delay discounting of real and hypothetical rewards III: steady-state assessments, forced-choice trials, and all real rewards. Behavioural processes, 69(2), 173-187. https://doi.org/10.1016/j.beproc.2005.02.003

Lamm, C., Zelazo, P. D., \& Lewis, M. D. (2006). Neural correlates of cognitive control in childhood and adolescence:Disentangling the contributions of age and executivefunction. Neuropsychologia, 44(11), 2139-2148. https://doi.org/10.1016/j.neuropsychologia.2005.10.013

Lavagnino, L., Arnone, D., Cao, B., Soares, J. C., \& Selvaraj, S. (2016). Inhibitory control in obesity and binge eating disorder: A systematic review and meta-analysis of neurocognitive and neuroimaging studies. Neuroscience \& Biobehavioral Reviews, 68, 714-726. https://doi. org/10.1016/j.neubiorev.2016.06.041

Lawrence, N. S., O'Sullivan, J., Parslow, D., Javaid, M., Adams, R. C., Chambers, C. D., ... Verbruggen, F. (2015). Training response inhibition to food is associated with weight loss and reduced energy intake. Appetite, 95, 17-28. https://doi.org/10.1016/j.appet.2015.06.009 
Liang, J., Matheson, B., Kaye, W., \& Boutelle, K. (2014). Neurocognitive correlates of obesity and obesity-related behaviors in children and adolescents. International Journal of Obesity, 38(4), 494-506. doi: 10.1038/ijo.2013.142

Liberman, N., \& Trope, Y. (2008). The psychology of transcending the here and now. Science, 322(5905), 1201-1205. doi: 10.1126/science.1161958

Lim, S. S., Vos, T., Flaxman, A. D., Danaei, G., Shibuya, K., Adair-Rohani, H., ... Andrews, K. G. (2013). A comparative risk assessment of burden of disease and injury attributable to 67 risk factors and risk factor clusters in 21 regions, 1990-2010: a systematic analysis for the Global Burden of Disease Study 2010. The Lancet, 380(9859), 2224-2260. https://doi.org/10.1016/S01406736(12)61766-8

Limbers, C. A., \& Young, D. (2015). Executive functions and consumption of fruits/vegetables and high saturated fat foods in young adults. Journal of health psychology, 20(5), 602-611. https:// doi.org/10.1177/1359105315573470

Lin, H., \& Epstein, L. H. (2014). Living in the moment: Effects of time perspective and emotional valence of episodic thinking on delay discounting. Behavioral neuroscience, 128(1), 12-17. http://psycnet.apa.org/doi/10.1037/a0035705

Loeber, S., Grosshans, M., Korucuoglu, O., Vollmert, C., Vollstädt-Klein, S., Schneider, S., ... Kiefer, F. (2012). Impairment of inhibitory control in response to food-associated cues and attentional bias of obese participants and normal-weight controls. International Journal of Obesity, 36(10), 1334-1339. doi:10.1038/ijo.2011.184

Logan, G. D., Cowan, W. B., \& Davis, K. A. (1984). On the ability to inhibit simple and choice reaction time responses: a model and a method. Journal of experimental psychology: human perception and performance, 10(2), 276-291. http://psycnet.apa.org/doi/10.1037/0096-1523.10.2.276

Logan, G. D., Schachar, R. J., \& Tannock, R. (1997). Impulsivity and inhibitory control. Psychological science, 8(1), 60-64. https://doi.org/10.1111/j.1467-9280.1997.tb00545.x

Lokken, K. L., Boeka, A. G., Yellumahanthi, K., Wesley, M., \& Clements, R. H. (2010). Cognitive performance of morbidly obese patients seeking bariatric surgery. The American Surgeon, 76(1), 55-59.

Lumsden, J., Edwards, E. A., Lawrence, N. S., Coyle, D., \& Munafò, M. R. (2016). Gamification of cognitive assessment and cognitive training: a systematic review of applications and efficacy. JMIR Serious Games, 4(2), e11. doi:10.2196/games.5888

Maayan, L., Hoogendoorn, C., Sweat, V., \& Convit, A. (2011). Disinhibited eating in obese adolescents is associated with orbitofrontal volume reductions and executive dysfunction. Obesity, 19(7), 1382-1387. doi: 10.1038/oby.2011.15

Madden, G. J., Begotka, A. M., Raiff, B. R., \& Kastern, L. L. (2003). Delay discounting of real and hypothetical rewards. Experimental and clinical psychopharmacology, 11(2), 139-145. http:// psycnet.apa.org/doi/10.1037/1064-1297.11.2.139

Madden, G. J., Bickel, W. K., \& Jacobs, E. A. (1999). Discounting of delayed rewards in opioiddependent outpatients: exponential or hyperbolic discounting functions? Experimental and clinical psychopharmacology, 7(3), 284-293.

Manasse, S. M., Espel, H. M., Forman, E. M., Ruocco, A. C., Juarascio, A. S., Butryn, M. L., . . Lowe, M. R. (2015). The independent and interacting effects of hedonic hunger and executive function on binge eating. Appetite, 89, 16-21. https://doi.org/10.1016/j.appet.2015.01.013 
Manasse, S. M., Flack, D., Dochat, C., Zhang, F., Butryn, M. L., \& Forman, E. M. (2017). Not so fast: The impact of impulsivity on weight loss varies by treatment type. Appetite, 113, 193-199. doi: 10.1016/j.appet.2017.02.042

Manasse, S. M., Juarascio, A. S., Forman, E. M., Berner, L. A., Butryn, M. L., \& Ruocco, A. C. (2014). Executive Functioning in Overweight Individuals with and without Loss-of-Control Eating. European Eating Disorders Review, 22(5), 373-377. doi: 10.1002/erv.2304

Mann, T., Tomiyama, A. J., Westling, E., Lew, A.-M., Samuels, B., \& Chatman, J. (2007). Medicare's search for effective obesity treatments: diets are not the answer. American psychologist, 62(3), 220-233. http://psycnet.apa.org/doi/10.1037/0003-066X.62.3.220

Manwaring, J. L., Green, L., Myerson, J., Strube, M. J., \& Wilfley, D. E. (2011). Discounting of various types of rewards by women with and without binge eating disorder: evidence for general rather than specific differences. The Psychological Record, 61(4), 561-582. https://doi. org/10.1007/BF03395777

Mazur, J. E. (1987). An adjusting procedure for studying delayed reinforcement. In M. L. Commons, J. E. Mazur, J. A. Nevin, \& H. Rachlin (Eds.), Quantitative analyses of behavior, Vol. 5. The effect of delay and of intervening events on reinforcement value (55-73). Hillsdale, NJ: Lawrence Erlbaum Associates, Inc.

McCallum, S. (2012). Gamification and serious games for personalized health. Stud Health Technol Inform, 177, 85-96. doi: 10.3233978-1-61499-069-7-85

McClelland, J., Dalton, B., Kekic, M., Bartholdy, S., Campbell, I. C., \& Schmidt, U. (2016). A systematic review of temporal discounting in eating disorders and obesity: Behavioural and neuroimaging findings. Neuroscience \& Biobehavioral Reviews, 71, 506-528. doi: https://doi. org/10.1016/j.neubiorev.2016.09.024

McClure, S. M., Laibson, D. I., Loewenstein, G., \& Cohen, J. D. (2004). Separate neural systems value immediate and delayed monetary rewards. Science, 306(5695), 503-507. doi: 10.1126/ science. 1100907

Melby-Lervåg, M., \& Hulme, C. (2013). Is working memory training effective? A meta-analytic review. Developmental psychology, 49(2), 270-291. doi: 10.1037/a0028228

Meule, A. (2016). Dieting and Food Cue-Related Working Memory Performance. Frontiers in psychology, 7, 1944. doi: https://doi.org/10.3389/fpsyg.2016.01944

Meule, A. (2017). Interactive effects between flexible and rigid control of eating behavior on body weight: a moderated serial multiple mediation model. Health Psychology Report, 5(4), 314322. https://doi.org/10.5114/hpr.2017.70206

Meule, A., \& Vögele, C. (2017). Grand Challenges in Eating Behavior Research: Preventing Weight Gain, Facilitating Long-Term Weight Maintenance. Frontiers in psychology, 8, 388. doi: 10.3389/ fpsyg.2017.00388

Miller, J., Schäffer, R., \& Hackley, S. A. (1991). Effects of preliminary information in a go versus no-go task. Acta psychologica, 76(3), 241-292. https://doi.org/10.1016/0001-6918(91)90022-R

Minear, M., \& Shah, P. (2008). Training and transfer effects in task switching. Memory \& cognition, 36(8), 1470-1483. https://doi.org/10.3758/MC.336.8.1470

Miyake, A., \& Friedman, N. P. (2012). The nature and organization of individual differences in executive functions four general conclusions. Current directions in psychological science, 21(1), 8-14. https://doi.org/10.1177/0963721411429458 
Miyake, A., Friedman, N. P., Emerson, M. J., Witzki, A. H., Howerter, A., \& Wager, T. D. (2000). The unity and diversity of executive functions and their contributions to complex "frontal lobe" tasks: A latent variable analysis. Cognitive psychology, 41(1), 49-100. https://doi.org/10.1006/ cogp.1999.0734

Mobbs, O., Iglesias, K., Golay, A., \& Van der Linden, M. (2011). Cognitive deficits in obese persons with and without binge eating disorder. Investigation using a mental flexibility task. Appetite, 57(1), 263-271. https://doi.org/10.1016/j.appet.2011.04.023

Moher, D., Hopewell, S., Schulz, K. F., Montori, V., Gøtzsche, P. C., Devereaux, P. J., . . . Altman, D. G. (2010). CONSORT 2010 Explanation and Elaboration: updated guidelines for reporting parallel group randomised trials. BMJ, 340, c869. doi: 10.1136/bmj.c869

Morrison, A. B., \& Chein, J. M. (2011). Does working memory training work? The promise and challenges of enhancing cognition by training working memory. Psychonomic Bulletin \& Review, 18(1), 46-60. https://doi.org/10.3758/s13423-010-0034-0

Mullan, B., Allom, V., Brogan, A., Kothe, E., \& Todd, J. (2014). Self-regulation and the intention behaviour gap. Exploring dietary behaviours in university students. Appetite, 73, 7-14. https:// doi.org/10.1016/j.appet.2013.10.010

Nederkoorn, C., Coelho, J. S., Guerrieri, R., Houben, K., \& Jansen, A. (2012). Specificity of the failure to inhibit responses in overweight children. Appetite, 59(2), 409-413. https://doi.org/10.1016/j. appet.2012.05.028

Nederkoorn, C., Jansen, E., Mulkens, S., \& Jansen, A. (2007). Impulsivity predicts treatment outcome in obese children. Behaviour Research and Therapy, 45(5), 1071-1075.

Nederkoorn, C., Smulders, F. T., Havermans, R. C., Roefs, A., \& Jansen, A. (2006). Impulsivity in obese women. Appetite, 47(2), 253-256. https://doi.org/10.1016/j.appet.2006.05.008

Ng, M., Fleming, T., Robinson, M., Thomson, B., Graetz, N., Margono, C., .. A Abera, S. F. (2014). Global, regional, and national prevalence of overweight and obesity in children and adults during 1980-2013: a systematic analysis for the Global Burden of Disease Study 2013. The Lancet, 384(9945), 766-781. https://doi.org/10.1016/S0140-6736(14)60460-8

Noël, X., Bechara, A., Dan, B., Hanak, C., \& Verbanck, P. (2007). Response inhibition deficit is involved in poor decision making under risk in nonamnesic individuals with alcoholism. Neuropsychology, 21(6), 778-786. doi: 10.1037/0894-4105.21.6.778

Nyhus, E., \& Barceló, F. (2009). The Wisconsin Card Sorting Test and the cognitive assessment of prefrontal executive functions: a critical update. Brain and cognition, 71(3), 437-451. https:// doi.org/10.1016/j.bandc.2009.03.005

O'Donnell, S., Daniel, T. O., \& Epstein, L. H. (2017). Does goal relevant episodic future thinking amplify the effect on delay discounting? Consciousness and cognition, 51, 10-16. https://doi. org/10.1016/j.concog.2017.02.014

O'Neill, J., Daniel, T. O., \& Epstein, L. H. (2016). Episodic future thinking reduces eating in a food court. Eating behaviors, 20, 9-13. https://doi.org/10.1016/j.eatbeh.2015.10.002

Odum, A. L. (2011). Delay discounting: trait variable? Behavioural processes, 87(1), 1-9. https://doi. org/10.1016/j.beproc.2011.02.007

Odum, A. L., Baumann, A. A., \& Rimington, D. D. (2006). Discounting of delayed hypothetical money and food: Effects of amount. Behavioural processes, 73(3), 278-284. https://doi.org/10.1016/j. beproc.2006.06.008 
Odum, A. L., \& Rainaud, C. P. (2003). Discounting of delayed hypothetical money, alcohol, and food. Behavioural processes, 64(3), 305-313. https://doi.org/10.1016/S0376-6357(03)00145-1

Oettingen, G. (2012). Future thought and behaviour change. European review of social psychology, 23(1), 1-63. https://doi.org/10.1080/10463283.2011.643698

Ohmura, Y., Takahashi, T., Kitamura, N., \& Wehr, P. (2006). Three-month stability of delay and probability discounting measures. Experimental and clinical psychopharmacology, 14(3), 318328. http://dx.doi.org/10.1037/1064-1297.14.3.318

Owen, A. M., McMillan, K. M., Laird, A. R., \& Bullmore, E. (2005). N-back working memory paradigm: A meta-analysis of normative functional neuroimaging studies. Human brain mapping, 25(1), 46-59. doi: 10.1002/hbm.20131

Perpiñá, C., Segura, M., \& Sánchez-Reales, S. (2016). Cognitive flexibility and decision-making in eating disorders and obesity. Eating and Weight Disorders-Studies on Anorexia, Bulimia and Obesity, 1-10. doi: 10.1007/s40519-016-0331-3

Peters, J., \& Büchel, C. (2010). Episodic future thinking reduces reward delay discounting through an enhancement of prefrontal-mediotemporal interactions. Neuron, 66(1), 138-148. doi: 10.1016/j.neuron.2010.03.026

Petrocelli, J. V. (2003). Factor validation of the consideration of future consequences scale: Evidence for a short version. The Journal of social psychology, 143(4), 405-413. https://doi. org/10.1080/00224540309598453

Petry, N. M. (2001). Delay discounting of money and alcohol in actively using alcoholics, currently abstinent alcoholics, and controls. Psychopharmacology, 154(3), 243-250. http://dx.doi. org/10.1007/s002130000638

Piko, B. F., \& Brassai, L. (2009). The role of individual and familial protective factors in adolescents' diet control. Journal of health psychology, 14(6), 810-819. https://doi. org/10.1177/1359105309338971

Prencipe, A., Kesek, A., Cohen, J., Lamm, C., Lewis, M. D., \& Zelazo, P. D. (2011). Development of hot and cool executive function during the transition to adolescence. Journal of experimental child psychology, 108(3), 621-637. doi: 10.1016/j.jecp.2010.09.008

Prickett, C., Brennan, L., \& Stolwyk, R. (2015). Examining the relationship between obesity and cognitive function: a systematic literature review. Obesity research \& clinical practice, 9(2), 93113. doi: 10.1016/j.orcp.2014.05.001

Raman, J., Hay, P., \& Smith, E. (2014). Manualised Cognitive Remediation Therapy for adult obesity: study protocol for a randomised controlled trial. Trials, 15(1), 426. https://doi. org/10.1186/1745-6215-15-426

Raman, J., Hay, P., Tchanturia, K., \& Smith, E. (2017). A randomised controlled trial of manualized cognitive remediation therapy in adult obesity. Appetite, 123, 269-279. https://doi. org/10.1016/j.appet.2017.12.023

Rappange, D. R., Brouwer, W. B., \& van Exel, N. J. A. (2009). Back to the consideration of future consequences scale: Time to reconsider? The Journal of social psychology, 149(5), 562-584.

Rasmussen, E. B., Lawyer, S. R., \& Reilly, W. (2010). Percent body fat is related to delay and probability discounting for food in humans. Behavioural processes, 83(1), 23-30. https://doi.org/10.1016/j. beproc.2009.09.001 
Ravussin, E., \& Bogardus, C. (2000). Energy balance and weight regulation: genetics versus environment. British Journal of Nutrition, 83(S1), S17-S20. https://doi.org/10.1017/ S0007114500000908

Reitan, R. M. (1958). Validity of the Trail Making Test as an indicator of organic brain damage. Perceptual and motor skills, 8(3), 271-276. https://doi.org/10.2466/pms.1958.8.3.271

Reitan, R.M.(1992). TrailMaking Test:Manualforadministration and scoring: Reitan Neuropsychology Laboratory.

Robinson, E., Haynes, A., Hardman, C. A., Kemps, E., Higgs, S., \& Jones, A. (2017). The bogus taste test: Validity as a measure of laboratory food intake. Appetite, 116, 223-231. https://doi. org/10.1016/j.appet.2017.05.002

Rollins, B. Y., Dearing, K. K., \& Epstein, L. H. (2010). Delay discounting moderates the effect of food reinforcement on energy intake among non-obese women. Appetite, 55(3), 420-425. doi: 10.1016/j.appet.2010.07.014

Roth, R. M., Isquith, P. K., \& Gioia, G. A. (2005). Behavior rating inventory of executive function--adult version (BRIEF-A). Lutz, FL: Psychological Assessment Resources

Sanchez-Cubillo, I., Perianez, J., Adrover-Roig, D., Rodriguez-Sanchez, J., Rios-Lago, M., Tirapu, J., \& Barcelo, F. (2009). Construct validity of the Trail Making Test: role of task-switching, working memory, inhibition/interference control, and visuomotor abilities. Journal of the International Neuropsychological Society, 15(3), 438-450. https://doi.org/10.1017/S1355617709090626

Schacter, D. L., Addis, D. R., \& Buckner, R. L. (2008). Episodic simulation of future events. Annals of the New York Academy of Sciences, 1124(1), 39-60. http://dx.doi.org/10.1196/annals.1440.001

Schacter, D. L., Benoit, R. G., \& Szpunar, K. K. (2017). Episodic future thinking: mechanisms and functions. Current opinion in behavioral sciences, 17(Supplement C), 41-50. doi: https://doi. org/10.1016/j.cobeha.2017.06.002

Schiff, S., Amodio, P., Testa, G., Nardi, M., Montagnese, S., Caregaro, L., . . S Sellitto, M. (2016). Impulsivity toward food reward is related to BMI: Evidence from intertemporal choice in obese and normal-weight individuals. Brain and cognition, 110, 112-119. https://doi.org/10.1016/j. bandc.2015.10.001

Schmeichel, B. J., Volokhov, R. N., \& Demaree, H. A. (2008). Working memory capacity and the self-regulation of emotional expression and experience. Journal of personality and social psychology, 95(6), 1526-1540. http://dx.doi.org/10.1037/a0013345

Schmiedek, F., Lövdén, M., \& Lindenberger, U. (2014). A task is a task is a task: putting complex span, n-back, and other working memory indicators in psychometric context. Frontiers in psychology, 5. 1475. https://doi.org/10.3389/fpsyg.2014.01475

Sellbom, K. S., \& Gunstad, J. (2012). Cognitive function and decline in obesity. Journal of Alzheimer's Disease, 30(s2), S89-S95. doi: 10.3233/JAD-2011-111073

Shallice, T., \& Vallar, G. (1990). The impairment of auditory-verbal short-term storage. Neuropsychological impairments of short-term memory, 11-53.

Sheeran, P. (2002). Intention-Behavior Relations: A Conceptual and Empirical Review. European review of social psychology, 12(1), 1-36. doi: 10.1080/14792772143000003

Sheffer, C. E., Mackillop, J., Fernandez, A., Christensen, D., Bickel, W. K., Johnson, M. W., ... Williams, J. (2016). Initial examination of priming tasks to decrease delay discounting. Behavioural processes, 128, 144-152. https://doi.org/10.1016/j.beproc.2016.05.002 
Shipstead, Z., Hicks, K. L., \& Engle, R. W. (2012). Cogmed working memory training: Does the evidence support the claims? Journal of Applied Research in Memory and Cognition, 1(3), 185193. https://doi.org/10.1016/j.jarmac.2012.06.003

Smith, E., Hay, P., Campbell, L., \& Trollor, J. (2011). A review of the association between obesity and cognitive function across the lifespan: implications for novel approaches to prevention and treatment. Obesity Reviews, 12(9), 740-755. http://dx.doi.org/10.1111/j.1467789X.2011.00920.X

Smith, E., Hay, P., \& Raman, J. (2014). Cognitive remediation therapy for obesity (176-191). Cognitive Remediation Therapy (CRT) for Eating and Weight Disorders, Routledge/Taylor \& Francis Group, New York, NY, US.

Spencer, E. A., Appleby, P. N., Davey, G. K., \& Key, T. J. (2002). Validity of self-reported height and weight in 4808 EPIC-Oxford participants. Public Health Nutr, 5(04), 561-565. https://doi. org/10.1079/PHN2001322

Spiegelman, B. M., \& Flier, J. S. (2001). Obesity and the regulation of energy balance. Cell, 104(4), 531-543. https://doi.org/10.1016/S0092-8674(01)00240-9

Spierer, L., Chavan, C. F., \& Manuel, A. L. (2013). Training-induced behavioral and brain plasticity in inhibitory control. Frontiers in human neuroscience, 7, 427. https://doi.org/10.3389/ fnhum.2013.00427

Spitznagel, M. B., Alosco, M., Strain, G., Devlin, M., Cohen, R., Paul, R., ... Gunstad, P. D. J. (2013). Cognitive function predicts 24-month weight loss success after bariatric surgery. Surgery for Obesity and Related Diseases, 9(5), 765-770. https://doi.org/10.1016/j.soard.2013.04.011

Spitznagel, M. B., Garcia, S., Miller, L. A., Strain, G., Devlin, M., Wing, R., . . Mitchell, J. E. (2013). Cognitive function predicts weight loss after bariatric surgery. Surgery for Obesity and Related Diseases, 9(3), 453-459. https://doi.org/10.1016/j.soard.2011.10.008

Stingl, K. T., Kullmann, S., Ketterer, C., Heni, M., Häring, H.-U., Fritsche, A., \& Preissl, H. (2012). Neuronal correlates of reduced memory performance in overweight subjects. Neuroimage, 60(1), 362-369. https://doi.org/10.1016/j.neuroimage.2011.12.012

Strack, F., \& Deutsch, R. (2004). Reflective and impulsive determinants of social behavior. Personality and social psychology review, 8(3), 220-247. https://doi.org/10.1207/s15327957pspr0803_1

Strathman, A., Gleicher, F., Boninger, D. S., \& Edwards, C. S. (1994). The consideration of future consequences: Weighing immediate and distant outcomes of behavior. Journal of personality and social psychology, 66(4), 742-752.

Stroop, J. R. (1935). Studies of interference in serial verbal reactions. Journal of experimental psychology, 18(6), 643-662. http://psycnet.apa.org/doi/10.1037/0096-3445.121.1.15

Svaldi, J., Brand, M., \& Tuschen-Caffier, B. (2010). Decision-making impairments in women with binge eating disorder. Appetite, 54(1), 84-92. https://doi.org/10.1016/j.appet.2009.09.010

Swinburn, B., Egger, G., \& Raza, F. (1999). Dissecting obesogenic environments: the development and application of a framework for identifying and prioritizing environmental interventions for obesity. Preventive medicine, 29(6), 563-570. https://doi.org/10.1006/pmed.1999.0585

Sze, Y. Y., Daniel, T. O., Kilanowski, C. K., Collins, R. L., \& Epstein, L. H. (2015). Web-based and mobile delivery of an episodic future thinking intervention for overweight and obese families: a feasibility study. JMIR mHealth and uHealth, 3(4). e97. doi: 10.2196/mhealth.4603

Tangney, J. P., Baumeister, R. F., \& Boone, A. L. (2004). High self-control predicts good adjustment, less pathology, better grades, and interpersonal success. Journal of personality, 72(2), 271-324. 
Tchanturia, K. (2014). Cognitive Remediation Therapy (CRT) for eating and weight disorders: Routledge.

Tchanturia, K., Giombini, L., Leppanen, J., \& Kinnaird, E. (2017). Evidence for Cognitive Remediation Therapy in Young People with Anorexia Nervosa: Systematic Review and Meta-analysis of the Literature. European Eating Disorders Review, 25(4), 227-236. doi: 10.1002/erv.2522

Tchanturia, K., Davies, H., Reeder, C., \& \&Wykes, T. (2010). Cognitive remediation therapy for anorexia nervosa. Retrieved from https://www.national.slam.nhs.uk/wp-content/uploads/2014/04/ Cognitive-remediation-therapy-for-Anorexia-Nervosa-Kate-Tchantura.pdf

Teixeira, P. J., Carraça, E. V., Marques, M. M., Rutter, H., Oppert, J.-M., De Bourdeaudhuij, I., .. Brug, J. (2015). Successful behavior change in obesity interventions in adults: a systematic review of self-regulation mediators. BMC medicine, 13(1), 84. https://doi.org/10.1186/s12916-0150323-6

Tiggemann, M., \& Kemps, E. (2005). The phenomenology of food cravings: the role of mental imagery. Appetite, 45(3), 305-313. https://doi.org/10.1016/j.appet.2005.06.004

Toepoel, V. (2010). Is consideration of future consequences a changeable construct? Personality and Individual Differences, 48(8), 951-956. https://doi.org/10.1016/j.paid.2010.02.029

Toplak, M. E., West, R. F., \& Stanovich, K. E. (2013). Practitioner Review: Do performance-based measures and ratings of executive function assess the same construct? Journal of Child Psychology and Psychiatry, 54(2), 131-143. http://dx.doi.org/10.1111/jcpp.12001

Uzogara, S. G. (2017). Obesity epidemic, medical and quality of life consequences: a review. International Journal of Public Health Research, 5(1), 1-12.

Vainik, U., Dagher, A., Dubé, L., \& Fellows, L. K. (2013). Neurobehavioural correlates of body mass index and eating behaviours in adults: a systematic review. Neuroscience \& Biobehavioral Reviews, 37(3), 279-299. https://doi.org/10.1016/j.neubiorev.2012.11.008

van Beek, J., Antonides, G., \& Handgraaf, M. J. (2013). Eat now, exercise later: The relation between consideration of immediate and future consequences and healthy behavior. Personality and Individual Differences, 54(6), 785-791. https://doi.org/10.1016/j.paid.2012.12.015

Van Strien, T., Frijters, J. E., Bergers, G., \& Defares, P. B. (1986). The Dutch Eating Behavior Questionnaire (DEBQ) for assessment of restrained, emotional, and external eating behavior. International Journal of Eating Disorders, 5(2), 295-315. http://dx.doi.org/10.1002/1098108X(198602)5:2<295::AID-EAT2260050209>3.0.CO;2-T

Veling, H., Aarts, H., \& Stroebe, W. (2013). Stop signals decrease choices for palatable foods through decreased food evaluation. Frontiers in psychology, 4, 875. https://doi.org/10.3389/ fpsyg.2013.00875

Veling, H., Lawrence, N. S., Chen, Z., van Koningsbruggen, G. M., \& Holland, R. W. (2017). What is trained during food go/no-go training? A review focusing on mechanisms and a research agenda. Current addiction reports, 4(1), 35-41. https://doi.org/10.1007/s40429-017-0131-5

Verbeke, G., \& Molenberghs, G. (2000). Linear mixed models for longitudinal data: Springer series in statistics. New York: Springer.

Verbruggen,F., Best,M., Bowditch,W.A.,Stevens,T.,\&McLaren,I.P.(2014).Theinhibitorycontrol reflex. Neuropsychologia, 65, 263-278. https://doi.org/10.1016/j.neuropsychologia.2014.08.014

Verbruggen, F., \& Logan, G. D. (2008). Automatic and controlled response inhibition: associative learning in the go/no-go and stop-signal paradigms. Journal of Experimental Psychology: General, 137(4), 649-672. http://dx.doi.org/10.1037/a0013170 
Verdejo-García, A., Pérez-Expósito, M., Schmidt-Río-Valle, J., Fernández-Serrano, M. J., Cruz, F., Pérez-García, M., .. . Marcos, A. (2010). Selective alterations within executive functions in adolescents with excess weight. Obesity, 18(8), 1572-1578. doi: 10.1038/oby.2009.475

Veronese, N., Facchini, S., Stubbs, B., Luchini, C., Solmi, M., Manzato, E., . . Fontana, L. (2017). Weight loss is associated with improvements in cognitive function among overweight and obese people: A systematic review and meta-analysis. Neuroscience \& Biobehavioral Reviews, 72, 87-94. https://doi.org/10.1016/j.neubiorev.2016.11.017

Volkow, N. D., Wang, G.-J., \& Baler, R. D. (2011). Reward, dopamine and the control of food intake: implications for obesity. Trends in cognitive sciences, 15(1), 37-46. https://doi.org/10.1016/j. tics.2010.11.001

Volkow, N. D., Wang, G. J., Telang, F., Fowler, J. S., Goldstein, R. Z., Alia-Klein, N., . . Ma, Y. (2009). Inverse association between $\mathrm{BMI}$ and prefrontal metabolic activity in healthy adults. Obesity, 17(1), 60-65. doi: 10.1038/oby.2008.469

von Bastian, C. C., \& Oberauer, K. (2013). Distinct transfer effects of training different facets of working memory capacity. Journal of Memory and Language, 69(1), 36-58. https://doi. org/10.1016/j.jml.2013.02.002

Voon, V. (2015). Cognitive biases in binge eating disorder: the hijacking of decision making. CNS spectrums, 20(6), 566-573. https://doi.org/10.1017/S1092852915000681

Vuchinich, R. E., \& Simpson, C. A. (1998). Hyperbolic temporal discounting in social drinkers and problem drinkers. Experimental and clinical psychopharmacology, 6(3), 292.

Wadden, T. A. (2003). Behavioral treatment of obesity. Endocrinology and Metabolism Clinics of North America, 32, 981-1003. https://doi.org/10.1016/S0889-8529(03)00072-0

Wadden, T. A., Butryn, M. L., \& Byrne, K. J. (2004). Efficacy of lifestyle modification for long-term weight control. Obesity, 12(S12), 151S-162S. doi: 10.1038/oby.2004.282

Weatherly, J. N., \& Derenne, A. (2011). Comparing delay discounting rates when using the fill-inthe-blank and multiple-choice methods. The Journal of general psychology, 138(4), 300-318. https://doi.org/10.1080/00221309.2011.606442

Weatherly, J. N., \& Ferraro, R. F. (2011). Executive functioning and delay discounting of four different outcomes in university students. Personality and Individual Differences, 51(2), 183187. https://doi.org/10.1016/j.paid.2011.03.042

Weatherly, J. N., \& Terrell, H. K. (2010). Delay discounting of different commodities II: Confirmatory analyses. The Journal of general psychology, 138(1), 35-48.

Webb, T. L., \& Sheeran, P. (2006). Does changing behavioral intentions engender behavior change? A meta-analysis of the experimental evidence. Psychological bulletin, 132(2), 249-268. doi: 10.1037/0033-2909.132.2.249

Weller, R. E., Cook III, E. W., Avsar, K. B., \& Cox, J. E. (2008). Obese women show greater delay discounting than healthy-weight women. Appetite, 51(3), 563-569. https://doi.org/10.1016/j. appet.2008.04.010

Wesley, M. J., \& Bickel, W. K. (2014). Remember the future II: meta-analyses and functional overlap of working memory and delay discounting. Biological psychiatry, 75(6), 435-448. https://doi. org/10.1016/j.biopsych.2013.08.008 
Westwood, H., Stahl, D., Mandy, W., \& Tchanturia, K. (2016). The set-shifting profiles of anorexia nervosa and autism spectrum disorder using the Wisconsin Card Sorting Test: a systematic review and meta-analysis. Psychological medicine, 46(9), 1809-1827. https://doi.org/10.1017/ S0033291716000581

Weygandt, M., Mai, K., Dommes, E., Leupelt, V., Hackmack, K., Kahnt, T., . . Haynes, J.-D. (2013). The role of neural impulse control mechanisms for dietary success in obesity. Neuroimage, 83(Supplement C), 669-678. doi: https://doi.org/10.1016/j.neuroimage.2013.07.028

Whitelock, V., Nouwen, A., van den Akker, O., \& Higgs, S. (2018). The role of working memory sub-components in food choice and dieting success. Appetite. 124, 24-32. https://doi. org/10.1016/j.appet.2017.05.043

World Health Organization (WHO) (2018). Obesity and overweight. 2018, retrieved from http:// www.who.int/mediacentre/factsheets/fs311/en/

Whyte, J., Polansky, M., Cavallucci, C., Fleming, M., Lhulier, J., \& Coslett, H. B. (1996). Inattentive behavior after traumatic brain injury. Journal of the International Neuropsychological Society, 2(4), 274-281. https://doi.org/10.1017/S1355617700001284

Willcutt, E. G., Doyle, A. E., Nigg, J. T., Faraone, S. V., \& Pennington, B. F. (2005). Validity of the executive function theory of attention-deficit/hyperactivity disorder: a meta-analytic review. Biological psychiatry, 57(11), 1336-1346. https://doi.org/10.1016/j.biopsych.2005.02.006

Wing, R. R. (2004). Behavioral approaches to the treatment of obesity. In: Bray, G. A., Bouchard, C. (eds.). Handbook of obesity: clinical applications (147-167). New York: Marcel Dekker.

Wing, R. R., \& Phelan, S. (2005). Long-term weight loss maintenance. The American journal of clinical nutrition, 82(1), 222S-225S. https://doi.org/10.1093/ajcn/82.1.222S

Wu, M., Brockmeyer, T., Hartmann, M., Skunde, M., Herzog, W., \& Friederich, H.-C. (2014). Setshifting ability across the spectrum of eating disorders and in overweight and obesity: a systematic review and meta-analysis. Psychological medicine, 44(16), 3365-3385. https://doi. org/10.1017/S0033291714000294

Wykes, T., Huddy, V., Cellard, C., McGurk, S. R., \& Czobor, P. (2011). A meta-analysis of cognitive remediation for schizophrenia: methodology and effect sizes. American Journal of Psychiatry, 168(5), 472-485. https://doi.org/10.1176/appi.ajp.2010.10060855

Yang, Y., Shields, G. S., Guo, C., \& Liu, Y. (2018). Executive function performance in obesity and overweight individuals: A meta-analysis and review. Neuroscience \& Biobehavioral Reviews, 84, 225-244. doi: https://doi.org/10.1016/j.neubiorev.2017.11.020

Yanovski, J. A., \& Yanovski, S. Z. (2003). Treatment of pediatric and adolescent obesity. Jama, 289(14), 1851-1853. doi:10.1001/jama.289.14.1851

Zinke, K., Einert, M., Pfennig, L., \& Kliegel, M. (2012). Plasticity of executive control through task switching training in adolescents. Frontiers in human neuroscience, 6. https://doi.org/10.3389/ fnhum.2012.00041 
